\title{
POETRY AND REASON: MEANS AND MEANINGS OF THE MOVING IMAGE
}

by

\author{
Iona Pelovska \\ MFA in Film Production, Concordia University, Montreal, 2001 \\ BFA in Film Production, Concordia University, Montreal, 1994
}

\author{
A dissertation \\ presented to Ryerson University and York University \\ in partial fulfilment for the degree of \\ Doctor of Philosophy \\ in the Program of \\ Communication and Culture
}

Toronto, Ontario, Canada 2015

(C) Iona Pelovska 2015 
I hereby declare that I am the sole author of this dissertation. This is a true copy of the dissertation, including any required final revisions, as accepted by my examiners.

I authorize Ryerson University to lend this dissertation to other institutions or individuals for the purpose of scholarly research.

I further authorize Ryerson University to reproduce this dissertation by photocopying or by other means, in total or in part, at the request of other institutions or individuals for the purpose of scholarly research.

I understand that my dissertation may be made electronically available to the public. 
(NOTE: the poem below is derived from the text of the Abstract by cutting out openings on the blank page preceding the Abstract.)

prosthetic

prosthetic
environments
beyond
perception,
language reveals
and dream.
of technology
solar

language

solar

and illuminate

Poetry

in body, unlike

art.

brings

sculpts

imperfect

being aims
sculpts

cinema. 
Poetry and Reason, PhD 2015, Iona Pelovska, Communication and Culture, Ryerson/York University

\section{Abstract}

Poetry and Reason departs from the question of cinema as industrial technology and as artistic language. The prosthetic relationship of industrial technologies to the body, unlike the contingency of traditional art technologies on the body, problematizes the question of cinema as art. Cinema's capacity to render thought-like environments allows its trans-mediumatic abstraction. This extends its questioning beyond the technologically cinematic, into the pre-technological moving image that animates perception, thought and dream. In scanning the field that makes cinema possible, this work questions the possibilities cinema opens as a way of knowing and assembling realities. The interpolation of language, thought and embodiment reveals a view of language as mediumatic, and of cinema as a linguistic medium that can simulate a cognitively faithful dream (the original disembodied moving image) as sensory experience. Thus cinema re-enacts a pre-technological environment while advancing the language of technology embedded in the cinematic machine.

The libidinal ways solar and chthonic energies, the symbolic and the physical, interpolate in mytho-poetic thought, art and science culminate in the ways these energies unfold in the experience of cinema and illuminate human hybridization, from ancient anthropo-bestiality to the techno-human condition. Poetry and reason, as the two ways of language, outline an epistemology that foregrounds the primacy of poetry in symbolic being. This work aims to resolve tensions between the symbolic and the material both theoretically and methodologically, proposing an integration of rational and poetic (artistic) techniques. The methodological intervention brings divergent approaches into tensional dynamic that sculpts a mobile structure, necessarily open-ended and imperfect. Theoretically, it delves into the movements of poetry and reason as ways to meaning, investigating their destination in cinema. The vocabulary of reason prompts cinema to advance a technological intent to colonize reality while poetic language destabilizes that movement. Reason and poetry as the two ways of language are thus actualized in the ways cinematic language interpolates mental and sensory experience. 


\section{Acknowledgements}

This work is a labour of tenacious research, merciless questioning and unconditional love. It had to be sustained through the onslaughts of reality and life's turbulence, and this is why it would not have been possible without the support of the people who stood by me during this process.

I would like to begin from the beginning by thanking Bruce Elder for inspiring me to embark on this journey and for his guidance in the early stages of my work. As a Rogers Fellow, I would also like to acknowledge the generous financial support of the Ted Rogers family.

Motivation is hardest to sustain during such a long process and for this my deepest gratitude goes to my committee. I feel extremely fortunate to have had such remarkable scholars overlook my work. Being guided by three people who embody their intellectual commitments, who walk their talk, gave me no excuse for abdication. I would like to give heartfelt thanks to my Supervisor, Caitlin Fisher, for having the courage to trust my process when I was doubtful and the vision to dispel my fear when I was stuck. My special gratitude goes to Steven Bailey for his unwavering presence in answering any question I would have with staggering erudition, kindness and precision. And finally, I am indebted to Michael Prokopow not only for his rigour and sense of beauty, but also for applying them to my text in a meticulous final edit that helped me submit this work as glitch-free as time would afford me. Thank you, thank you, thank you!

Lost in thought and time, it was the Department Secretary, Jo Ann Mackie, who helped me keep my life on track when I had wandered off in the wilderness of my mind. I would like to thank her for her endless kindness and ruthlessness in kicking me in action at the exact moment of need. I would also like to acknowledge the Department Chairs Paul Moore and Izabella Pruska-Oldenhof for their invaluable administrative and time management support.

The trepidation I felt during this journey was immense and I wouldn't have made it without the emotional support of family and friends. Many heartfelt thanks go to Anne Wordsworth for her 
beautiful friendship, for making her house my home and for bringing Walter Benjamin's Arcades Project to my attention. Special thanks to Aileen Weaver for her graceful touch, her unwavering emotional support and for driving all the way from Arizona to see me during the last stretch of the marathon. Many thanks to Christine Korte for the inspiring conversations and the sense of wonder and joy with which she imbued my academic life. Finally, I would like to thank Lyuba Encheva who has been my co-conspirator in tackling life's mysterious meanderings through academic work.

I would like to give special thanks to my ex-husband, Grigor Kuzmanov, who threw a few wrenches in my wheels early in the research process, allowing my work to mature and deepen in the delay. He has been my intellectual companion for years and he continues to be one of the most insightful critics and supporters of my thought. He showed me how to think with my heart and for this I will always be grateful.

Elizabeth Trott has helped me survive not only financially, by repeatedly employing me as her Assistant, but also mentally, by offering her wise council, warm friendship and remarkable sense of humour whenever I needed them. Thank you for sharing your brilliance so generously!

Finally, my deepest thanks go to my partner Thibaud De Corta who has been my rock during the last two years of intense writing. Without his loving patience and unwavering support I wouldn't have had the conditions to finish this work. He provided me with the peace and stability needed for uninterrupted discent into thought, while making sure I didn't lose myself in the process. His belief in my work, his emotional and intellectual insight, and above all, his selfless presence have made the successful completion of my dissertation the only option. Thank you! 
In memory of my grandmother and namesake, Iona Pelovska, a remarkable being whose intellectual honesty has nurtured the love of knowledge and the sense of wonder that have inspired and sustained this work. 


\section{Table of Contents}

Abstract Poem iii

Abstract $\quad$ iv

$\begin{array}{ll}\text { Acknowledgements } & \mathbf{v}\end{array}$

Dedication vii

Introduction $\quad 1$

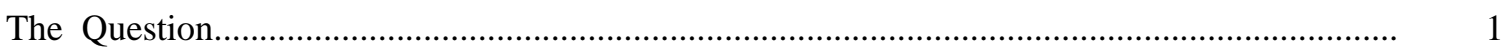

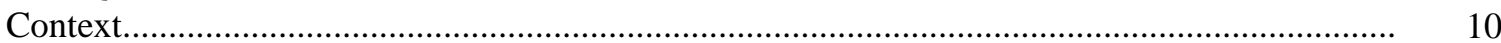

$\begin{array}{ll}\text { Chapter 1. Poetry and Reason: Methodological Considerations } & 17\end{array}$

$\begin{array}{ll}\text { Chapter 2. Method } & 63\end{array}$

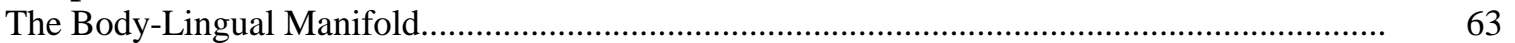

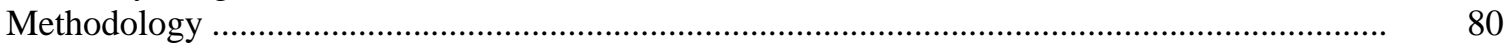

Chapter 3. Mind-Body and the Cinematic Machine 101

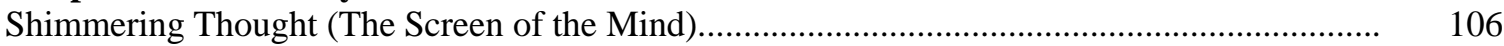

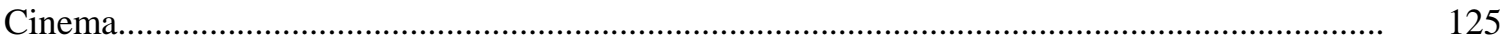

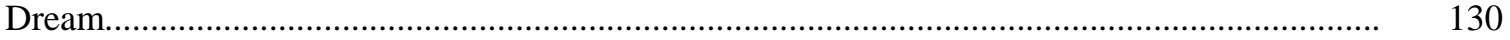

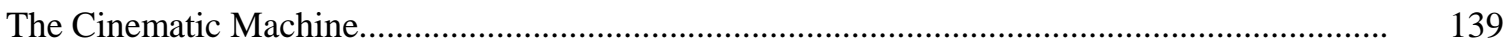

Chapter 4. Myth Prophecy and Technology 149

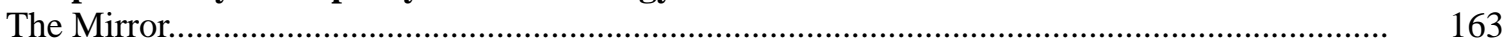

Chapter 5. Art: Diffractions of Technology and the Body 175

Chapter 6. The Body of Cinematic Technology 201

$\begin{array}{ll}\text { Chapter 7. Poetry and Pornography } & 213\end{array}$

$\begin{array}{ll}\text { Conclusion } & 233\end{array}$

$\begin{array}{ll}\text { Bibliography } & 249\end{array}$

$\begin{array}{lr}\text { Appendix } & 258\end{array}$

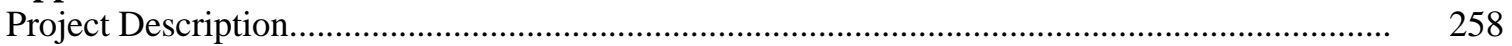

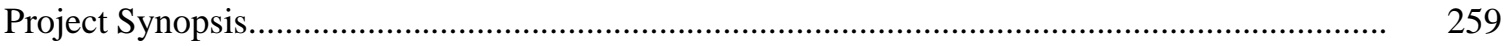

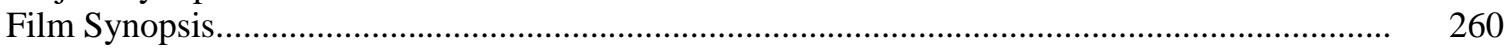




\section{Introduction}

\section{The Question}

It could be said that research is a quest that begins with a question. And often it is the question that foretells whether the journey would be a short walk on the beach or an open-sea sail. The question allows the quest to take us to a new place or circle us back to the point of departure. Some questions might seem restricting, like "Can I get to India by travelling West?" but end up taking us to places we haven't even imagined existed, to pre-Columbian America. Other questions might be stated openly - "What can I be certain of?" - but betray a pre-conceived idea that there is an "I" that is so stable as to be entitled to a self-sustaining certainty.

“...when I analyze the event expressed in the sentence 'I think' I acquire a series of rash assertions which are difficult, perhaps impossible, to prove - for example, that it is I which thinks, that it has to be a something at all which thinks that thinking is an activity and operation on the part of an entity thought of as it has cause, that an 'I' exists, finally that what is designated by 'thinking' has already been determined -- that I know what thinking is..." (Nietzsche, 2003 [1886]: 28)

The answer to such an "open" question, not surprisingly, circles back to itself, to the thinking-questioning "I" - "I think, therefore I am." The questions of a research enterprise are, therefore, as important as the answers they may be set to unveil. That is to say, even the most basic questions are lodged in prejudice, be it just a linguistic one. Answers, in turn, by offering a closure to a question, endanger to obscure as much as they enlighten by foregrounding only one side of the manifold of the questioned. Instead of questions and answers as beginnings and ends, I propose the use of questions and answers as a way to converse with things and negotiate passages. And this will conveniently provide an open answer to the open question: 
"Is it necessary "to begin," and, if so, is it necessary to start from a point of view of a subjective certainty?" (Deleuze, Guattari, 2003 [1991]: 27)

So, it is not the question that is as important as the quest this question entails - the impulse to move towards something both known (as it is already hinted, it has shown its edge to us) and unknown (as it has also retrieved itself, so it is there with us and yet it is nowhere in sight). It is therefore the quest in the questioning, the direction the mind turns to, that sets up a research in search of the mysteries that beckon from the edge of the known, because "...the true stance of thinking cannot be to put questions, but must be to listen to that which our questioning vouchsafes - and all questioning begins to be a questioning only in virtue of pursuing its quest..." (Heidegger, 1982 [1971]: 72)

The present work is fuelled by a curiosity about the cinematic medium, that is to say the media of the moving image. As a filmmaker with a background in Fine Arts, I have been consistently unsettled by the possible implications of the medium in which I had chosen to work. Cinema has emerged as an industrial technology that seems, throughout its advent, to both liberate and conflict, to varying degrees, with the traditional artistic techniques. Liberate, because it allows the artist to delegate mechanical tasks to the machine and focus on the poetic aspect of the work; conflict because firstly, the poetic emerges in an intricate holistic process that includes the mechanical artistic gesture and secondly, because in delegating the work to an industrial machine, the artist both enacts a mechanical separation of labour and poetic work and makes his or her physical relation to the work optional (replaceable by the machine). This made the term art film, the field I have been working in, loaded with fuzzy contradictions. While traditional art media had been discussed and refined for centuries of artistic practice and discourse, cinema, along with all industrial and hyper-industrial media, seems to have been almost uncritically embraced by artists both enthused and intimidated by the steel authorities of progress and 
efficiency. Cinema brought together all traditional artistic disciplines in a united front at the advent of a techno-scientific era that threatened to obliterate the arts as a cognitive field. At the same time cinematic equipment itself, the tools of the field, threatened to replace the artist and foreground the technology as the agent of communication in a process of "pernicious industrialization of vision" (Virilio 1997, 89). This can be traced from the first screenings of the simple daily sequences the Lumiere brothers called actualites such as Workers Leaving the Lumiere Factory (1895) or Arrival of the Train at la Ciotat Station (1895) filmed and shown as spectacles of technological wonder to the current focus of the film industry on special effect technologies, most notably hailed in comparatively recent 3-D animations that hit the top of the box office list such as Avatar (2009) and Frozen (2013). Moreover, while cinematic technologies, especially with the advent of sound film (replacing live sound accompaniment), promised to reconcile the millennial cognitive competition between the ear and the eye (McLuhan 1964, 4849), they also threatened to hijack those faculties and disembody them in ways never experienced before, and satirically summed up in "The Man Who Fell to Earth" (1976) where an alien is destabilized by the hypnotic effect of television and, abandoning his mission, spends his time watching a wall of active TV screens. In the early $20^{\text {th }}$ century modernist hopes and anxieties, the intimate play between the human condition and cinema technologies inspired the mechanistic dreams of the Futurists, seeing the perfection of the machine reflected in the future human while at the same time fuelling nightmarish thoughts about the next techno-evolutionary mutation:

"When a child goes to the movies... he suffers psychic damage from the form of the presentations alone, regardless of their content. The cinema may be quite "decent"... But the sheer fact that the viewer becomes habituated to the flashing, fluttering, and twitching images of the flickering screen slowly but surely destroys his psychic ... stability. [...] The cinema is constructing a new human type...: the homo cinematicus." (Kaes 2009, 224) 
In short, my quest in the land of cinema revealed sights and sounds that formed the questions of this research - questions that were not only posed by me to cinema, but also by cinema to me, where often my own answers would "take me by surprise and teach me what I think," as Merleau-Ponty had allegedly observed. (Derrida, 1978 [1967]: 11) Thus, the questioning of cinema, with which this work is concerned, is a manifold conversation, not a unilateral interrogation. Yet, it starts with a single question: What does the cinematic medium make possible, or advance, and what does it obscure, or preclude? This question of departure is a simplified version of McLuhan's tetrad of media effects (what does a medium enhance, what it reverses, what it retrieves and what it makes obsolescent). The tetrad plays into McLuhan's dynamics of figure and ground whereby, given a certain shift of perspective and/or condition, a figure can become ground and vice versa: the medium, when enhancing, is a figure but it becomes ground when it sends something into obsolescence. (McLuhan, 1992 [1988]) For instance, while cinema amplifies the audio-visual, such as in the re-enactment of a story (hitherto confined to theatre), it is also the ground of driving away corporeal presencing (also a defining feature of theatre). Thus cinema could be regarded as the contextual ground for a mediumatic and experiential movement away from physicality. The spatio-temporal interjection of cinema is explored at length in the thought axis of Benjamin - Virilio: cinema technology as altering the basis of human experience - space-time - technology as alien and alienating. But the question is where in the manifold of human experience space-time becomes a necessary notion and how it relates to technology. More primary still is the question where did cinema technology abide before the Lumiere brothers inaugurated our physical relationship with it.

But how could anything physical move away from its physicality? And more precisely, how could anything physical move away from its physicality without dying? Technology enables this by offering a mobile accommodation of the symbolic further and further away from human 
embodiment. In its capacity to assemble new layers of reality, cinema, more than any technological medium, has dramatized the dangers and promises of this movement away/toward physicality as "the intensity of automatic weaponry and the new capacities of photographic equipment combine to project a final image of the world, a world in the throes of dematerialization and eventual total disintegration..." (Virilio1989, 73)

Paul Virilio's anxieties about the alienating advent of new media technologies, inaugurated by cinema, descend from Benjamin's critique of industrial technologies. For Benjamin, human perception was challenged by the insertion of the cinematic in the perceptual field, which collapsed space-time, making everything equidistant and up close to the viewer. (Banjamin 1940-1088). For Virilio, while traditional cinema has been naturalized as visual extension, it was telecommunication that finalized the new media process of disrupting the continuity between sign and object by making the sign increasingly independent and mobile, into transforming matter into mobile signs that ultimately collapse space-time into a radically different alien modality of light-speed (Virilio 1997). The replacement of time and space with light and speed Virilio attributes to the alien and alienating character of the techno-scientific project which obliterates human experience by privileging a virtual "lumiocentrism." Light obliterates duration (the temporality of embodied experience) in its instantaneous speed of information transmission. Taking away space-time as the scene of luminosity, and instead replacing the former with the latter, disturbs human modes of perception. Virilio sees cinema as the technological beginning of this process of defying human vision, as the first technology that would capture phenomena ungraspable by the naked eye (e.g. slow motion), a process concluded by virtual technology in the establishment of luminocentrism. However, space-time itself is a construct of the technoscientific model - in order to dissolve into a hyletic experience it first requires a level of mental abstraction that can introduce the concept to the mind-scape and eventually reabsorb it, 
restructure perceptual interpretation so to speak. Such conceptually restructured perception inevitably acquires an extra degree of detachment, beginning the alienation process tracked in the works of Benjamin and Virilio. The current work, while taking into account the Benjamin-Virilio view of cinematic technologies, sets out to explore the techno-scientific modality not only as a socio-cultural phenomenon that is alien and alienating but also as a way of articulating that enters human experience in ways that make it symbiotic with the latter. How does the pre-technological human, the way of the body, conduct the mediumship that conditioned the development and naturalization of technology in general and cinematic technologies, in particular?

This brings up the question of the cinematic medium and its relations to reality, a question which has been central to film theory since the emergence of cinema as photography-inmovement, i.e. as a spacial object recording playing out in time. When in 1890 the Lumiere brothers invented their camera-projector they called it the cinematograph, from the Greek kinema (movement) and graphein (to write) - a machine that would write down movement and replay it, much like an illuminated text that has come to life in front of the ecstatic gaze of the reader. Cinema is thus defined by the moving image in so far as it is the technological intervention that brings forth mediation of the image in movement. Speaking of the cinematic thus necessarily implies the moving image and its relationship to reality.

The realist theories of Andre Bazin and later Stanley Cavell privileged the photographic image in cinema as ontologically committed to reality the way a mold is committed to the original as "no matter how fuzzy, distorted or discoloured, no matter how lacking in documentary value the image may be, it shares, by virtue of the very process of its becoming, the being of the model of which it is the reproduction." (Bazin 1967, 14) Cavell advanced this reality argument further by observing that "physical molds and impressions and imprints have clear procedures for getting rid of their originals, whereas in a photograph, the original is still as present as it ever was." 
(Cavell 1979, 20) This realist commitment to cinema privileges photographed live action and either regards animation as the fulfilment of abstract visual arts (Bazin) or as a problematic phenomenon on the margins of cinema (Cavell):

"There is one whole region of film which seems to satisfy my concerns with understanding the special powers of film but which explicitly has nothing to do with projections of the real world - the region of animated cartoons... If this region of film counters my insistence upon the projection of reality as essential to the medium of the movies, then it counters it completely." (Cavell 1979, 167)

Cavell goes on to argue that, based on its relationship to reality, the relationship of animation to cinema is akin to the difference between animal species. His ontology derives from a relationship to reality that privileges the physical/witnessed to the mental/imagined in a dichotomy that, while foregrounding valid and important considerations, underestimates cinema as a domain of artifact and art work which, as such, "always entails the creation of new spaces and times" and is "not a question of recounting a story in a well-determined space and time; rather, it is the rhythms, the lighting, and the space-times themselves that must become the true characters." (Deleuze, in Flaxman 2000, 370) This new autonomous reality is necessarily aligned through a new language:

"A work of art is a new syntax, one that is much more important than vocabulary and that excavates a foreign language in language.” (Deleuze, in Flaxman 2000, 370)

Deleuzian cinema is thus not a particular reality-bound species but a kind of new life. Thus he turns the lens of cinema to life rather than reality, foregrounding time as its driving force. In his theory of cinema, he extends Bergson's ideas of the image as a constituent of matter defined by its duration (as the totality of its time), unspacialized by analyzable freeze frames or moments. This flow of the Bergsonian image allows for a holistic movement of the different elements that comprise the cinematic manifold, such as frame, shot, sound. Thus, instead of the conceptual 
compartmentalization of cinematic time-space, Deleuze offers the continuous flow of the timeimage and movement-image, two radically new ways to see the image as lived-temporal and not merely spacio-representational. Unlike Bergson who sees film as conceptualizing time, spacializing it into immobile frames, aligning it with perception and thus obfuscating the more complex and more full experience of the (durational) image (Bergson 1911 [1929]), Deleuze sees film as the movement of images beyond representation, movement as organic content uniquely cinematic, which could be either a perception-based cinematic life, a movement-image (Deleuze 1986 [1997]), or a technology-based cinematic thought (Deleuze 1989 [1997]). However, both the spacial-realist axis Bazin-Cavell and the temporal-aesthetic one Bergson-Deleuze, focus on cinema as the media and language that have already been forged into existence. The 24 framesper-second film-time of Bergson, for example, cannot intimate the nuanced temporality variations of magnetic recording or digital code, let alone non-technological ways of experiencing moving image temporalities that may be related to the cinematic in ways that bypass a specific medium. Deleuze's example-driven approach precludes him from probing the pre-cinematic interpolations of life and thought that may fold or unfold into the cinematic. Similar parameters bind the film semiotics of Metz, with its focus on film-form, as well as to the cognitive film theory of Bordwell inevitably concerned with mental representation as that which constitutes meaning. This filmevidence-based theoretical framework has precluded film theorists from asking questions about cinema that have not already been answered, in a way, by cinema itself. My questions are concerned with the phenomenon of cinema as always already emergent in a techno-linguistic environment that makes it both possible as mediumatic language and distinct from any other way of communication/knowing.

As communication medium, cinema is contingent on cognition, i.e. on how it unfolds in perception and thought. This leads to the question of the moving image as a (pre-technological) 
category of thought, a predicative experience toward a cognitive coming into language, and its relationship to the (thus externalized) moving image of cinematic technologies on one hand, or the mental moving image as structured by the technological imperative that brings it to the movie screen, on the other. This latter angle on the question brings together the Kantian non-predicative nature of existence (i.e. the existence of the mental moving image does not prioritize it to the cinematic moving image) with the Lyotardian insistence on the predicative position of technology vis a vis humanity. So, the first layer of questioning that would allow us to approach the moving image would investigate how does the moving image emerge in awareness and how does it play out in pre-cinematic experience. This would mean questioning the process of experiencing the moving image in perception and thought on one hand, and on the other, reviewing a range of thoughts and findings on the question, including neuroscientific and cognitive research that has contributed to the way we might think the thinking process vis a vis the moving image. The Deleuzian view of the work of cinema discussed earlier foregrounds the integral relationship between reality and language, which is echoed in a neuroscientific view of embodiment and the mind:

"For centuries philosophers have assumed that (the) gap between brain and mind poses a deep epistemological problem - a barrier that simply cannot be crossed. (...) I'd like to argue that there is in fact no such barrier... Indeed, I believe that this barrier is only apparent and that it arises as a result of language. This sort of obstacle emerges when there is any translation from one language to another." (Ramachandran 1999 [1998], 231)

However, while in (cinema) art language is reality, in (neuroscientific) reality language dissects it, forging gaps between its layers. This counter point intimates the interpolations that emerge by cross-examining the question of experience through different disciplinary lenses and opens into the second layer of questioning which foregrounds the cinematic medium as technology. How the technological moving image interplays with human awareness or rather, 
how does the technological moving image encounter its cognitive double? In the course of this cyclical layering, the questions would remain as open as possible so that the ship of this research may bump into unploughed shores, without losing sight of the goal - what does it mean, cinema.

\section{Context}

From television to personal computers, from cinema to computer games, we are surrounded by second degree reality that plays on our perception of limitations and possibilities. What makes this reality so convincing, so life-like, is the moving image. When the imagined becomes an image it is actualized, but when the image becomes the imagined, the boundaries of possibilities begin to blur. The Cartesian mind only sees itself; that is, it only sees thought, and when it gets its feedback from its own product, i.e. thought feeding on thought, it locks down its understanding of the possible into a solipsistic universe. Losing the corporeal means losing a reference point for the boundaries of the imagined. Reality morphs from appearance to apparition in a blink of a thought. This loss is actualized by the scientific hold of the objective - physicality eschatologically appropriated by thought. The millennial battle of the symbolic human with bestiality promises to finally come to a victorious end. From the first recorded technological gesture, the fig leaf covering the bodies of Adam and Eve, to cybertextuality, corporeality has been progressively clothed in layers of symbolic interpretation to the point of almost complete obfuscation. Technologies, piling mediumatic layers on top of each other, have alienated thought from the body by entangling it in interpretations of interpretations - mediumships about mediumships - an endless stream of mediating, and medio-cratizing, that which has already mediated. Thus protected, physical reality is then attacked by the side of technology that was meant to violate. From the earliest found Oldowan tool to nuclear fission it has been pounded, 
pressured and chipped away into an impotent pile of components ready at hand to serve the increasingly ghostly human.

The dissolution of physical reality into a disembodied mind scape could be witnessed in the running sights captured and replayed by the cinematic moving image - a technological corollary of the symbolic thrust. The rational collapse of the (subjectively experienced) body into an object circuited into the internalization of the object-ive by thought finds a bona fide reflection in the cinematographic power to objectify physical reality into a string of reflections which are then spliced up in ways closely reminiscent of the (internal) processes of thought. Cinema's relationship to thought can thus be examined through the relationship between two cinematic conditions (in the sense of conditions of movement) - the time-based image and the imagined as always already in movement. How does this relationship reflect upon our perception of reality, and more specifically, our understanding of the body as constitutive of a reality? In other words, how have moving image technologies affected our understanding of the mind-body dynamic, its possibilities and its limitations?

These questions demand a rigorous examination of notions such as reality, mind, body, language, that have hitherto been axiomatic, or automatic, in film theory which tends to adopt terminology, approaches and techniques from other disciplines without necessarily examining their most basic premises. This study goes beyond such cross-disciplinary applications in film theory, summoning both ancient ideas and latest research in excavating the grounds for the emergence of core ideas and modes of thinking that have made cinema possible, and have been in turn, pollinated by it.

As the mediumatic apex of industrial technologies, cinema has opened new avenues of knowing the world - both scientific and artistic. Moving image documentation has annihilated irreversible time hitherto confounded to memory. Endoscopic cameras have turned the body 
inside out rendering the invisible visible. The discontinuous time of film editing has brought dream vision to wakeful reality. Memory, the hidden (insides of the) body and dream have all been brought into the light of social visibility out from the hermetic depths of the personal. What has breaking those barriers meant to the post-industrial human in his or her understanding of physical parameters as both cognitive closings and openings, as guardians of knowledge? To answer these questions means to look at technology and the human mind/embodiment in crossdisciplinary discourse - how have philosophy, science and art approached, framed and assembled their respective ideas, and what would be the implications for cinema, and vice versa.

The context of this research is defined by the cultural collision of a techno-biological imperative on one hand, and a perceived freedom of the subjective on the other, which has generated a string of cultural paradoxes. A focus on the problems of corporeality, the shifting presence of the body, in relation to visual time-based media within Occidental social paradigms aims to identify underlying principles in the development of media technology and media language that reflect and affect current cognitive parameters, and their social validation. How does a technology designed to liberate generate dependence? When does the will to understand converge with the desire to control? Where does a body caught between its formatting to proliferate and its (suicidal) will to free itself of any formatting reside?

In short, this research invites a meditation on the questions that cinema reanimates and re-problematizes in ways specific to no other techno-linguistic field. Moreover, it extends this meditation to that which cinema opens up and makes possible, that which Daniel Frampton, following in Deleuze's footsteps of looking at cinema as a singular non-mimetic phenomenon, identifies as filmind. "The coherent film-world is thus given a layer of intention in the work of film-thinking. The pre-reflective creation of the film-world (filmind thinking) is reflected upon 
(filmind Thinking). (...) Thus the filmind simultaneously creates and refigures the film-world." (Frampton 2006, 18-19)

However, while a significant part of this research is dedicated to the problem of the mind and cinema and cinema as mind, I do not refer to filmind further in the text, not only because I got acquainted with Frampton's work at a rather late stage of my own work, but also because I am interested, as already discussed, in the forces and movements that make possible and are made possible by cinema, forces and movements that both transcend and interpolate with the cinematic, both as mimesis and as singularity. Those problems are more accurately positioned on the cusp of philosophical questioning, artistic and scientific research. To bring together those three ways in a systematic and organic collaboration, I have crafted out a methodological intervention that aspires to give justice to all perspectives. In doing so, I search for as much original referencing as possible, meaning that, for example, in artistic research I refer to the works and writings of artists, in scientific one, I foreground scientists' writings and findings, in philosophy, I go to the origins of the philosophical ideas, and in all of them I sometimes go as far as the dawn o civilization and the first recorded documents of the emergence of certain ideas and practices.

Like most philosophical questioning, this investigation is rooted in the paradox of a symbolic animal perpetually torn between the power of being an alive yet limited physical body and, at the same time, a linguistic being able to freely roam past physical limitations. This drama is most explicitly unleashed in the human attempt to reconcile the power of embodiment with symbolic mobility in the development of industrial and post-industrial technologies. But the troubled relationship of human and technology, the drama of linguistic mind and physical body, is not a true dichotomy - embodied being does not recognize itself as limited, it is through symbolic language that we can think in terms of being beyond being, in terms of separations and dichotomies. Thus the paradox of embodiment and symbolic language is a problem that belongs 
to language. This is where the investigative lines of the current work converge, tracking down the two ways, of reason and poetry, through which language assembles and reveals itself in thought.

Some of the implications of reason and poetry as linguistic energies have been addressed in Dialectic of Enlightenment where Adorno and Horkheimer explore the relationship of their socio-cultural incarnations as enlightenment and myth. By foregrounding the techno-scientific context in which enlightenment and myth play out their dialectic entanglement Adorno and Horkheimer initiate a critique of historical reason as an ouroboric power that regresses cyclically into myth and vice versa. I take off from a similar position, that rational and mytho-poetic thought are indeed entangled, but I find that entanglement more originary and open-ended than the strategies of the two energies to devour each other. And even if that entanglement is nothing but spiral in pattern, both the rational and the mytho-poetic ways are still unfolding, projecting possible futures that do not necessarily converge. However, I share Adorno and Horkheimer's commitment to thought as that which is both actively displaced by and, indeed, the other of mechanization. This research therefore takes an unapologetic plunge into the theory that Adorno and Horkheimer see as endangered by modern developments:

"Today, however, motorized history is rushing ahead of such intellectual developments, and the official spokesmen, who have other concerns, are liquidating the theory to which they owe their place in the sun *before it has time to prostitute itself completely.*

In reflecting on its own guilt, therefore, thought finds itself deprived not only of the affirmative reference to science and everyday phenomena but also of the conceptual language of opposition. No terms are available which do not tend toward complicity with the prevailing intellectual trends, and what threadbare language cannot achieve on its own is precisely made good by the social machinery." (Horkeheimer, Adorno 2002 [1947], xv)

The theory I advance is not a seamless insert into centuries of theoretic discourse - before unfolding as a theory of cinema, it is both a methodological intervention and a meticulous examination of certain axiomatic assumptions sustaining the traditional theoretical complex. This 
intervention intends to realign the ideas behind those assumptions in new ways made apparent only through the unfoldment of the industrial techno-human entanglement as evidenced in cinema. Grounded in the idea of the empirical character of thought, this work foregrounds the ways language interfaces the physical and the mental, and the ways the two discipline each other through language in assembling the reality we collectively share. In chapter 3 I discuss how the rational itself, as that which has been most unequivocally assigned to thought, is grounded in and interpolated with empirical reality; how reason is not native to thought but rather, is thought disciplined by (physical) experience and vice versa, thought itself disciplines experience. In fact, this work locates thought in its state most independent from physicality not in rational abstraction, but in the irrational flow of dreams and reveries. In search for a hyletic screen of experience, a priomordial region of irreducible (in symbolic analysis) data in which representation emerges, Chapters 1,2 and 3 foreground a new way of interpreting reality that calls for a more theoretical cinema and a more cinematic thought, a more thoughtful art and a more artful thought. Chapter 4 and 5 plunge us into lived symbolic thought - culture. From mythology to art practices, it examines ways in which culture has played out the techno-symbolic entanglement. How has culture generated meanings about embodiment-language-technology that have resonated into and from the cinematic experience. Chapter 6 ties it all together into the materiality of cinema - how does a medium built on the alienation of embodiment materialize itself and how does it, in turn generate meaning. The last question brings us to cinema language and Chapter 7 makes a full circle back to the initial (Chapter 1) question of poetry and reason as they unfold in cinematic language.

Methodologically, this places the focus on thought as experience, and on language as the empirical way of theory, the way thought becomes flesh, and grounds itself in stable (linguistic) form. Thus, throughout this work, I use language, the fabric of symbolic being, as a way as well 
as a material, a way that reveals its answers in the immersion and interaction with its material, similar to the ways clay or paint conjure realities in the hands of the artist. The methodology I introduce has emerged from my own artistic practice as a dialogue with the mediumatic language of my art. As traditionally trained visual artist, I have been inspired by the tactile enmeshment with the art materials. As a filmmaker, I have been informed by the ways cinematic art demands the synergistic mobilization of different modalities - the rational, the mechanical, the poetic. My use of traditional animation and painting techniques in cinema has sharpened the contrast between traditional and industrial ways of making art, informing both my methodology and my analysis of technology. However, I do not base my analysis on examples of my own work because my questioning goes beyond discursive knowledge, to the place where language itself becomes an empirical act that frames discursive knowledge itself. I find this place of empirical language not where language distances itself from experience in conceptual synthesis, but where it abandons itself to poetry. This is why it is in dialogue between the poetic and the conceptual that I conduct my investigation. In doing this, I intend to invoke dance, that most physical of art forms, into the movement of theory. 


\section{Chapter 1. Poetry and Reason: Methodological Considerations}

"In the beginning, I adored. What I adored was human. Not persons; not totalities, not defined and named beings. But signs. Flashes of being that glanced off me, kindling me. Lightening-like bursts that came to me: Look! I blazed up. And the sign withdrew. Vanished. While I burned on and consumed myself wholly. What had reached me, so powerfully cast from a human body, was Beauty: there was a face, with all the mysteries inscribed and preserved on it; I was before it, I sensed that there was a beyond, to which I did not have access, an unlimited place. The look incited me and also forbade me to enter; I was outside, in a state of animal watchfulness. A desire was seeking its home. I was that desire. I was that question."

Hélène Cixous. Coming to Writing

Using a single methodology, or a set of methods that subscribe to the same paradigm, could promote solipsistic reasoning and tautological (self-fulfilling) findings, especially in research focused on complex phenomena that refer to a number of different cognitive orders (respectively, bound to a variety of linguistic structuring). This raises the question of language as a closed system that expresses its own conventions and ways to conjure up meaning, i.e. as a system that reflects upon itself (Wittgenstein). The positivist proposition that empirically unverifiable linguistic statements are nonsensical still runs into the wall of the phenomenological proposition that the perceived becomes knowable/thinkable through language, which in effect places empirical reality in the same linguistic cognitive loop. At the other end of the scientific spectrum stands the Platonic/Pythagorean standard - the ideal language of mathematics as the safeguard of reality - the real is the measurable, anything beyond measurability is unreal. Measurability thus includes not only empirical reality, the domain of the body, but also symbolic reality as far as a symbol is a (necessarily bound, hence measurable) unit of information. In other words, form as the measurable, is real. However, the photon which arguably has zero mass, hence formless, must lie at the edge of the scientifically knowable - the particle-wave that behaves as the still measurable (1 photon) but elusively formless reference to the detectable universe. It is the 
formless light that gives form to the (measurable) universe. Thus light marks not only the threshold of Einsteinian Physics (as per the famous e=Mc2), of visible reality, but it also guards the boundary of sensible reality, the reality of both form and mass, matter and perception, art and science. Or does it?

Certain forms remain stubbornly detached from mass or a measurable reference - a dream apple is just that, a dream apple. It appears morphs or disappears for the inner eye of the dreamer only. The dream apple is not illuminated by photons, it is visual and yet it abides somewhere beyond the rays of light, it is beyond the grasp of a scientific instrument or even beyond the grasp of visceral instrumentation, such as the hand grasp of the dreamer. It is not even one apple, as it may appear many times simultaneously, or not even have the shape of an actual apple. It does not exist and yet, it is there, it is part of the dreamer's world as much as a physical apple may be, reverberating in ways that may be just as semantically far-reaching. It is strikingly reminiscent of Plato's ideal of form with one major difference - it is not ideal. It is as unique, flawed and finicky as the dreamer may be. So, strictly speaking, measurability grants reality to the form that corresponds to another form (belonging to another formal, causally related, order) the shape of an apple apprehended by the eyes corresponds to a physical object of an apple, touchable, edible and measurable by a range of (formed) instruments. A non-oneiric apple is a network of measurable correspondences made possible by a mirror-like understanding of the mind as reflective of the world. Therefore, the truth of science, as Heidegger observed, is a truth of correct correspondences. (Heidegger 1977 [1943]) Furthermore, the more correspondences can be established for a single phenomenon, the more real it is. Therefore, science rests on a notion of language as correspondence.

Art as a field of human enterprise is concerned primarily with form, all form, the measurable and the elusive. In fact, it could be said that it emerges from the immeasurable, from 
the dream world itself, from the unquantifiable domain of human experience that lies at the edge of science. Art also grounds its quest in correspondences. The academic art student begins by learning to draw an apple as faithfully as possible, to grasp the principles of its appearance as thoroughly as the cognitive capacities of the human body would allow. S/he is concerned with figuring out the optical principles of light and perspective that inform the eye there is a physical entity corresponding to the (apprehended) visual form. In other words, the student is concerned with learning the principles of formal correspondences - how does light reflect (visibly) from different volumes and textures, how does the geometry of physical space appear to the eye. The art student learns to detach the shape of bodies, to snatch them away from their original physicality and let them float to a piece of paper, reminiscent of the way they float, disembodied, in dream space. Except, once caught, they are pinned to the piece of paper through the artist's skill, then tied to the (measurable) physicality of the artistic medium - the paper and paint. The art student is a student of language as correspondence.

Thus far the symbolic endeavours of humanity, that is those endeavours concerned with language in its widest sense, seem both in pursuit of correspondences. But this is only partially correct - the pursuit of correspondences is the way to untangle the mesh of appearances toward under-standing them, toward revealing what stands under them, what upholds them. But that presupposes that there is a "something" behind any-thing. In other words, they were concerned with that which was not directly apparent: science with the undetectable by the common instruments of the body, and the arts with the incommunicable by the correspondences of language. But the question "what" presupposed the expectation to uncover "something" behind the veils of the apparent world.

To evade the totalizing danger embedded in the question "what," i.e. to evade the enframing of metaphysics, science has resorted to foregrounding the question "how" addressing 
the dynamic of relationships that define a phenomenon's boundaries, its limitations and possibilities. To evade the objectifying limitations of metaphysics, the arts have also foregrounded the how as addressing the process of envisioning. The resulting shift to foregrounding language and its mechanisms was not confined to the visual arts, it spread to literature and music. Thus, the shift from visibility to the way of envisioning in the visual arts was not a visual process. But this shift of focus of the symbolic enterprise on process, a shift that may arguably be ascribed to Kant's turning the proverbial telescope of enquiry from the observed to the observer, still remains within the same domain, the domain of observed and observer, of subject and object, of physical and symbolic reality - a domain that belongs to conceptual thinking.

"What something is... whatness, comprises since Plato what one commonly calls the "nature" or essentia, the essence of a thing. Essence so understood becomes restricted to what is later called the concept, the idea or mental representation by means of which we propose to ourselves and grasp what a thing is." (Heidegger, 1982 [1971]: 94)

Concept is a linguistic function, while Heideggerian thingness is the flesh of word. Therefore concept and thing emerge in different (temporal) realms - word and object are not related, neither can thing and object, nor word and concept be reduced to one another. In the domain of concept and object, the two remain both interchangeable and unrelated, while in the domain of word and thing, the two lead to one another, fuse and yet remain uniquely singular in their standing. The object restricting thingness is an offshoot of science that has promoted the idea that language, as we speak it, reflects reality. The concept restricting the word is a scientific contamination into the arts that promotes the confusion that language, as we speak it, begets reality. When is the former an idea and the latter a confusion? Whenever the universe, as an expanse of conceptually organized objects, is the domain of scientific research, the domain of 
logical and techno-logical organization, the domain that uses language to map, measure and probe objects that are there "standing reserve" (Heidegger 1977 [1954]) to be seized by technology. In other words, conceptual thinking belongs to science. The world as the domain that works with language to arrive at every-thing as a radiant singularity remains the domain of art. To subjugate art to concept, to the linearity of the conceptual world is therefore a scientific contamination that endangers the entire artistic domain.

My use of the word "world" is deliberate and not interchangeable with "universe". The "world" here means the knowable universe as humanly apprehended and interpreted. In a way, we could say that the world begins with the first sensation, the sensation of being here as a singular point of feeling. I derive this understanding of the word from both the English and (my native) Bulgarian etymologies. The English word is rooted in the Germanic "wer" (man), alluding to an anthropocentric expanse that presumably begins and ends with the limits of human apprehension. It includes all that appears to the human, including dreams, phantoms and imaginations. The Bulgarian "sviat" means "light" and, quite directly suggests a manifest or illuminated part of the universe, an expanse that has emerged into a state of unconcealment. It begins with the first demiurgic words: "Let there be Light," and the first singular sensation of being, that is, of unconcealment, of emerging in the wordness of light. It is interesting to note that the word "sviat" remains relevant with Einsteinian physics and its reliance on light as a point of physical departure. It also relates to Bohmian holomovement as "the complex movement of electromagnetic fields, in the form of light waves." In other words, "sviat" points toward both Einsteinian intergalactic universe and Bohmian particle universe. The Bohmian definition also implies a line of divergence between the world and the universe - "the totality of movement of enfoldment and unfoldment may go immensely beyond what has revealed itself to our observations thus far" (Bohm 2002, 225). 
The word "universe" comes from the Latin unus (one) and vertere (to turn), i.e. unified in movement and refers to the Greek $\pi \varepsilon \rho \iota \varphi о \rho \alpha$ (something transported in a circle) which reflects an ancient Greek model of matter as moving in concentric circles. The Bulgarian word "vselena" is a composite of the Old Slavonic words for "everything" and "occupy, inhabit" - all that inhabits/occupies, the occupied continuum - and refers to the Greek oikov $\mu \varepsilon^{\prime} \eta$, meaning "to occupy, to inhabit." Therefore, Bohmian holomovement fits both of these words as referring to everything that inhabits and moves - the (spatial) continuum of (temporal) movement.

A look at the Old Slavonic for "world" (mir) and "universe" (vsemir) would provide another entry into the current distinction between the two. The word "mir" means peace and could be related to the original Greek meaning of the word cosmos (kó $\sigma \mu \rho)$ ) as "order." "Vsemir" means "all-peace," or "all the world," that which is the world now, and that which also includes the world as we don't (yet) know/envision, but can be or may have been envisioned. This more original reference of the word "universe" to all that is and isn't (manifest), is twofold - on one end it cracks an opening into that which is beyond human grasp, the unknown, it extends the human sphere beyond itself and/or beyond any single consolidation of human understanding (world). On the other hand, it brings that expanse into one. That oneness is thinkable as part of the world but not imaginable or knowable as part of the supra-human universe. Science has found an interesting solution to this epistemological limitation - the objectification of the universe thus observable via instruments that reside and detect outside of the human, instruments that not only augment the human body, but more currently, they constitute independent bodies with supra-human capacities. But in order to be knowable via such instruments, the universe had to be reduced to one not as in "whole", but as in "common denominator" which finds its most adequate incarnation in the concept of the object. The universe of objects becomes the object-ive universe of science. The Earth of the world becomes the Planet Earth of the universe. 
The world, on the other hand, begins with that which is already shining in the human field. It is there that the quest for knowledge begins, as an impulse to incorporate the sensible. It is an impulse to consolidate one's body in the world - only when the strange environment into which the human being is thrown becomes the known world, does the impulse to knowledge become a drive to surpass one's own body, one's own world. This quest for knowledge in the world has two ways. The first is the way of envisioning the universe as a reduction of a manageable expanse of objects/concepts that are incorporated into the existing (metaphysical) language so that it can bring back the unknown into the world as a communicable information package. The second is the way of pushing the boundaries of the world so that it generates new ways of including the strange and the alien hovering at its borders, it is a way that travels on language as a vessel that brings it all the way to the borders with the known, allowing the unknown to seep through its cracks and expand it. The first way maps the known and organizes it in clear linear pathways that streamline the conceptually packed unknown into familiar systems. It is the way of reason. The second way is the way of finding openings that lead from the familiar into the strange, that crack the skin of the world open and venture beyond the comprehensible, bringing language along as far as it can expand and reinvent itself. This is the way of poetry. The two are intertwined, not opposed.

The thought of the unknown is part of the world, but the unknown as a concept referring to a potential object of observation is a part of the universe. If the universe can include the unknown as a thinkable supposition, it becomes part of the world, which in turn is part of the universe as the expanse that includes the thinkable. We thus arrive at a Moebius strip of understanding being as flowing, folding and unfolding unto itself. The universe can be the subject of the world as far as the world accounts for the very idea of the universe as a thinkable proposition; the world cannot be the subject of the universe unless it is objectified, i.e. reduced to 
one of its components. Because, as already discussed, the universe of science deals with universals - those knowables that can be verified by more than one (measurable) correspondence - the world of objects and objective processes. It could be said that the universe is larger than humanity while the world is human, but the universe can only arise as a proposition within the fabric of the world. An epistemological endeavour is thus by definition initiated in the universe, but a cognitive one emerges in the world, and the two cannot exhaust any question unless they account for the other. At the simplest, most immediately articulate level, the world and the universe intertwine in the interplay of logic and imagination. When the art student masters the principles of visual rendition through drawing and painting from live models, then $\mathrm{s} / \mathrm{he}$ can begin rendering imaginary objects. Those imaginary objects are still part of the universe as objectified and conceptualized, but they are beginning to walk the line of the familiar as the student pulls out of the sensible world and begins to conjure possibilities. In short, the student begins with a flight of imagination (the generation of non-sensible images) and renders the vision through logic - the procedure of drawing or painting pictures as sensible objects. The student is still entrenched within the familiar, this is only a foretaste of the possibility to travel to the edge of the world. Conversely, the science student, once familiarized with the laws of sensible reality, e.g. with Newtonian physics, is led, through the logical path of formulas to the edge of the observable universe and into the Einsteinian imaginary. It is through logic that the art student returns from the domain of imagination, it is through logic that the science student enters into the domain of imagination. Both logic and imagination are integral to the cognitive process - logic as the stabilizer of the known, and imagination as the jump toward the knowable; conversely, logic travels beyond the sensibly known, invading the knowable, while imagination brings it back home, to the mind-body. This is only the beginning of the quest for the students of art and science. 
It could then be said that, while art opens the world, science penetrates the universe. In addition to the strangely sexual imagery that suddenly emerged from that sentence, it also casts a ray on how the two ways generate meaning. The Proto Indo-European "men-" is the stem of the verb "to think" in a number of European languages. In English too when "I mean" something, I point to the thinking that I cast in language. Thus, in the most general sense, "meaning" refers to information that has passed through thought, information that has been thought. It invokes not only the process of thinking but also thought as a kind of originary medium. This significance of the word "meaning" is retained in the English noun "mean" as both middle ("the mean of") and an intermediary tool ("by means of"). Thus understood, meaning refers to that which has been made explicit through thought, but not necessarily by reason. Thought and reason have often been used interchangeably, but they do not overlap. While reason is a form of thought, thought as considered in the present questioning, is a field larger than reason. In "On the Way to Language," Heidegger discusses poetry and thought as the two neighbouring fields that unfold with language, as the "two kinds of utterance par excellence." (Heidegger 1982 [1971], 80) He delineates the rational/scientific way of thinking from thought itself:

"In the sciences, not only is the theme drafted, called up by the method, it is also set up within the method and remains within the framework of the method, subordinated to it. [...] In thinking there is neither method nor theme, but rather the region, so called because it gives its realm and free reign to what thinking is given to think." (Heidegger 1982 [1971], 74)

The current quest is grounded in such a broad view of thought - a view of a field that generates different ways to meaning, science and art being two such ways, i.e. two ways to manifest through thought. But thought overflows the angular categories of Western metaphysics its way to manifestation passes through the body; it is through the feeling of embodiment that thought arrives to itself. A distinct thought emerges in the vast and vague field of thought as a resonance of something, a sensation - the more precisely a sensation is captured and transferred 
into the thought-field, the more vibrant and energetically charged the field becomes. We call this animated field of thought imagination, but this word may be misleading as it so loudly speaks of the priority of vision in the Western sensorium. But imagination is hardly all there is to this movement of thought toward sensory acquisition, toward becoming flesh. "Our thinking should have a vigorous fragrance, like a wheatfield on a summer's night," urges Nietzsche (qtd. Heidegger [1982] 1971, 70) toward a more sensual palpable mindfulness. Our thinking, to be true in this full-fleshed sense, should not only emanate the fragrance of its bloom but also, with matching vigour, should give off the stink of its decay. Meaning, as considered here, is therefore not only the passing of information through thought, the internalizing of form, the making of flesh into thought so to speak, but also the passing of thought through form, the lived experience of thought, the making of thought into flesh. This twofold movement of thought/flesh-form can be approached metaphysically only in necessarily fragmentary ways, such as through the conceptualization of disciplinary approaches (e.g. arts and sciences), of various methods and practices. It could be said that the arts explore the intricacies of this movement, bring it into attention, by foregrounding meaning as embodied thought - by focusing on the singularity of an instance, the timelessness of being, beauty. This is to say that the language of art is a language of experience. The sciences, on the other hand, are concerned primarily with language as conceptualization, with the movement of form through thought, the retention of data into conceptual packages, as per Logan's notions of language discussed further (Logan 2007). Needless to say, both sciences and arts enter and play in each other's language domains in separations by degrees that cannot allow an amputation of either from the body of language itself. They are the shadows cast by that full body of linguistic thought, but they may, conversely, guide us back to that body's ways, or openings, to listen and hear its whispers. Language is, therefore, 
not a mere sum of its sides, but the shining forth of the field of thought as animated, set forth, by the agglutinating force of logos:

"The word is logos. It speaks simultaneously as the name for Being and for Saying." (Heidegger [1971] 1982, 80)

In ancient Greece "logos" was commonly used to refer to an organizing principle of being, accessible to human thinking:

"For this reason it is necessary to follow what is common. But although the LOGOS is common, most people live as if they had their own private understanding.” (Graham 2011)

The logos, thus thought of and handed down to us by Heraclitus, is revealed before (private) interpretation, it is that which grounds human understanding as such.

The word "logos" is rooted in the Greek $\lambda \dot{\varepsilon} \gamma \omega$, which has a number of meanings - to lay down, to put to sleep; to put in order, arrange, gather; to choose, count, reckon; to say, speak, converse. These significations conjure up an inception of the world in the range of foregrounded meanings discussed earlier, as the domain of articulated (or articulable) being, as the Heideggerian "name for Being and Saying," or as the Biblical word as flesh. Therefore, logos as that which emerges first in thought does not, strictly speaking, belong to thought - rather, it is granted to it. It is that which emerges in thought, yet is not generated by thought, when thought is mobilized as thinking - the assembling principle of a micro-eschatology whereby the world begins with a single pull of focus, a listening to a saying:

"...the listening we have now in mind tends toward the grant, as Saying to which the nature of language is akin. [...] ...thinking is a listening to the grant." (Heidegger [1971] 1982, 76)

Thus cognition begins with a single act of listening, of pulling one's attentional focus toward a whisper of being. But the incarnated logos as that which mobilizes the field of thought is no longer the singular Word of God conjuring the universe from an unfathomable expanse, it is 
now that which guides being into thinking and thinking into being so that logos, that which is conjured from the abyss as some-thing, becomes language:

"Only where the word for the thing has been found is the thing a thing. [...] The being of anything that is resides in the word... Language is the House of Being." ( Heidegger [1971] 1982, $62-63)$

"Thing" is therefore, in a sense, the proto-word within which all things find their singular thingness. The constant flow of experience is slowed down by the focus the word affords. Rationality appears within the 'house of being" and on the field of thought, as a way of orienting only after the word has already afforded the (slowed down) realm that makes specialization possible. But, once again, orienting is not rationality - it is both the condition of rationality and that which rationality can identify as a foundational principle of the biological way of knowing conditioning the emergence of language as a spatial tool:

"Linguistic behaviour is orienting behaviour; it orients the orientee within his cognitive domain to interactions that are independent of the nature of the orienting interactions themselves.” (Maturana \& Varela [1972] 1980, 30)

Language as orienting behaviour is, according to Maturana, preceding Logan's language as a synthesizing or transporting tool (Logan 2007):

"If it is recognized that language is connotative rather than denotative and that its function is to orient the orientee within his cognitive domain, and not to point to independent entities, it becomes apparent that learned orienting interactions embody a function of non-linguistic origin that, under a selective pressure for recursive application, can originate through evolution the system of cooperative consensual interactions between organisms that is natural language." (Maturana \& Varela [1972] 1980, 30-31)

Maturana's permutation foregrounds the pre-symbolic, embodied, experience of language as sensory signalization. Thus placed, connotation turns from an effect of polysemic symbolization to signalization in sensory communication. Denotation, in turn, loses its indexical 
positioning as identification and inaugurates signification. That is to say, the word detaches from the form (of a thing) and becomes a sign that can potentially shift into a concept (of an object). Language, thus released, can extend communication through time and space, but only at the expense of the new gap it has opened between itself and things, a new communication space that needs to be minded if we are not to fall through this gap when, instead of trying to connect concepts, try to reach things with words. But saying that, what Maturana calls natural language precedes language as a symbolic system is just as misleading as saying that language determines experience, not because those are not valid statements but because they prioritize a particular temporal permutation, and temporal prioritization itself operates only in the conceptual modality of language - the modality of (linear) space-time. For instance, connotation reappears later in the linear unravelling of language when a concept becomes a symbol and can thus encompass a range of connotations, referring to phenomena beyond the object it represents. Likewise, denotation could be thought of as pre-perceptual packing of information, such as DNA encoding. Thus, prioritizing in the linear modality of thinking can only be conditional as searching for a conceptual "first" is bound to an endless loop synthesized in the familiar statement "This sentence is false." Moreover, we can experience conceptual language but conceptual language cannot induce anything beyond this experience of itself - we can experience the thought of space travel but not the sensation of space travel. Even when we are undergoing a synchronous experience such as sipping warm coffee from a porcelain cup, the moment we reflect on the experience conceptually, to the extent we do this, we seize to experience it sensually, and instead begin to experience its mental reflection. This power of conceptual language to hijack embodiment, turning the shapes of moments from distinct to disconnected, and the shapes of things from signature to sign, can obfuscate the power of natural language to assemble the world by providing a way of orienting from a particular point of awareness: 
"If we look into ourselves and consider experience, we discover that the contents of consciousness are not fixed forms with unchanging properties. Rather, what we experience is in perpetual flux. All that is fixed is that which linguistic energies themselves create. It is words that create fixity; we hang on to the word because of its stabilizing effects. However, words belong to the mind itself, and they are incommensurate with anything outside language. Certainly, we can summon words back to consciousness, and when they return to consciousness they can reanimate the experience with which they were previously associated. But a word does not represent an extramental reality or denote the experience with which it is associated - it is associated with it, and that is all. Language is completely autonomous of any possible extramental world." (Elder 2010,11)

And yet, Heidegger insists that the "being of anything that is resides in the word," that the word is what sustains thingness and isness. How can then language be strictly mental if any-thing that is is in language? Would that mean that the mental is some-thing embedded in language and then, conversely, channelling language on its terms - a sort of reversible universe, like a twosided jacket? Or would that mean that anything that is is a mental reality, as Descartes would have it? And that language is a free-association system of insignia connecting mental representations? Would that then mean that things, as mental realities conjured by the word, cannot be encountered as powerful presences in the external world, "however phantasmatic the externality of that world may be theorized to be?" (Brown 2001, 2) This conundrum would persist (as it often has) if we remained in the confines of conceptual thought where spatial demarcations of externalinternal/here-there held sway. And even if we don't want to give up the conveniences that conceptual language affords, the prioritization of the mental, either as experience or as language, is not final. The slowing down or stabilizing effect the single focus of attention, and language, afford while indeed "internal," happen before the consolidation of that focus of attention into an individual self, and there is no need to demonstrate this beyond the argument that, in the linear progression of things, such stabilization of something is the condition of the consolidation of anything. That is to say that the spacialization of a human being as a separate being whose body acts 
as the interface between an "internal" experience and and "external" encounter happens within an expanse that is necessary for such a spacialization to become possible. Thus a single focus of attention, a particular human being for instance, could be thought of as the assemblage point of energies (within that expanse that could become spacial) that pass through that point, not contained within it. In this case the mental could not be something else to the carnal, nor some inside space hermetically sealed from anything that may extend beyond it and vice versa; it is instead the mediumatic screen on which the intensification of those energies is articulated ( $a$ corporeal sign of a world-universe). This articulation would happen on the axis perceptionconception-language conducting and generated by the temporal spacialization of a (mediumatic) body-mind. Although "linguistic energies" would make this consolidation possible, in their capacity to, quite simply, single out and relate (i.e. communicate), language as a symbolic system could only unfold as the final element in that axis. In other words, symbolic language, the language that has the full capacity to become Logan's suitcase of concepts, can only crystallize when the process of perception-conception is well under way and the consolidation of the bodymind inaugurated. In order for it to become a fully symbolic system, it has to detach itself from the indexical embodiment of pointings and grunts (connotation as orientation), from signals and signs (denotation as identification) and project itself into the mental screen of conceptualization that has no specific associations and formal correlates. It has to become a free-standing articulation layered upon, but not energetically related to, perception as the primary mode of outside-in channelling.

This conceptualization of language begins even before language itself in the sense that a pre-verbal child already recognizes separate things and, in doing this, is already preparing to separate, hold and carry-over the (mental) representations of specific things through time-space. The destination of perception as symbolic language, layering over perceptual reality like a 
portable blanket, allows for imagining and constructing parallel free-standing realities that can safely entertain versions - it allows for the construction of artifice. Artifice in turn, brings forth two possibilities unique to the human condition - deception and making. Indeed, they are the two sides of symbolic language as it builds free-standing structures that circumvent the principles it borrows from the reality it claims to either represent or uphold. Moreover, it claims to exhaust and uphold phenomena that, as language-in-use, it has partial, associative and referential relationship to. But this claim is no proclamation, it is the overwhelming implication of its power to stabilize thought as the screen of sensory input, the latter elaborated by Nietzsche:

"I shall set apart, with great respect, the name of Heraclitus. When the rest of the philosophic populace rejected the evidence of the senses because they showed multiplicity and change, he rejected their testimony because they showed things as if they had duration and unity. Heraclitus too did the senses an injustice. They do not lie either in the way that the Eleatics believed, or as he believed they do not lie at all. What we make of their evidence is what gives rise to the lie, for example, the lie of unity, the lie of materiality, of substance, of duration... "Reason" is what causes us to falsify the evidence of the senses. If the senses show becoming, passing away, and change, they do not lie... But Heraclitus will always be right that Being is an empty fiction. The "apparent" world is the only one: the "real" world has only been lied on..." (Nietzsche 1998 [1889], 3)

The "real" is thus but a word that can only denote its own stabilizing character, whether it is associated with the stabilizing effect of perception-conception or with fully realized symbolic consolidation. The "linguistic energies" that stabilize thought are the same energies that stabilize perception on one hand, and mature in the craft of reason, on the other. This threefold consolidation allows for the word to sustain the thingness of things without being intrinsically related to any-thing, and language to operate independently in a reality that is held captive by linguistic principles (and by principles I mean the laws of physics as much as the changing grammar of a specific live language). Indeed, language is viewed here in its broadest sense, as the spirit of any interpretation, including the most primary interpretation - the perceptual. In the realm of forms (the threefold of presentation/representation/word), symbolic language, is the 
most formally arbitrary and hence, the most potent falsifier. Language, in order to be truthful, has to fully embrace its deceptive powers.

The free-standing, travelling, distancing, transgressive character of language affiliates it with vision as the most far-reaching sense, making images be at home in language. This is why representation, even when tactile, olfactory or sonic (as in the neuroscientific sense of mental representation), is still embedded in a visual word and, in turn, why vision has been culturally prioritized throughout the rational unfolding of language. It is also the reason I just constructed this view of body-mind as a reality-consolidating medium generating and generated by language as a way of intensifying energy through form that crystallizes in symbolic language as a blanket of sensual reality. Indeed, anything short of such view is bound to disregard (and often even attempts to deny) a variable host of (accounted for) phenomena, locking itself in loops of controversies, contradictions and conflicts. In addition, my use of visual language is a deliberate attempt to acknowledge the affinity of vision and language in ways that do not circumvent the possibility for defamiliarizing the distancing character of the visual and the linguistic afords.

Words may not denote any extramental reality, as far as reality beyond our grasp cannot be accessed by the mental, but they still connote an extramental expanse, mysterious and unknown, a realm that prevents the hermetic seal that the exclusivity of a singular point of awareness is in perpetual danger of executing. The orienting power of natural language (generating syntax) is therefore not only tied to the stabilizing effect of the word and thus, in sustaining the world, but also it points to the end of this world as a destination of being with language. Language can bring us to that edge, but this is also where it crumbles.

Keeping all that in perspective, when we find ourselves wandering in space-time, rationality provides the most reliable way of orienting on the travelled path. Linear tools of logic are a guaranteed way of navigating a terrain; just like extending a thread to mark one's trail is one 
of the surest ways to prevent getting lost in the wilderness. The word "reason" signifies that which underlies or precedes something else "the reason for," and points at its focus on causative relationships. The Proto-Indo-European root "re(i)-" means both "reason" and "count," thus engaging (causally) relational and computational thinking. Thus understood, "reason" encompasses the linear proximate ways of orienting in the terrain of language, isolating patterns (count) and establishing relationships. To exercise reason, one has to be already in a terrain, must have already been granted the language within which to orient. Reason helps us build trails in the wilderness of language. Therefore, to be reasonable means, before anything else, recognizing oneself as an entity in a space-time continuum of here-there, now-then, inner-outer, of being able to count to two and see form as a binary principle where McLuhlan's interplay between figure and ground becomes possible. That is, to think meta-physically, the conceptual detachment of the figure from the ground that reason, as a way of thinking in language, affords - reason as the destination of conceptual language; conceptual language as the destination of denotationconnotation (denotation-connotation as the destination of perceptual isolation-orientation).

However, the path is not (necessarily) the way.

Poetry is not concerned with orientation in a terrain. It is a much faster and less safe way to reach the end of the world as the destination of language. In poetry there is no necessary spacetime, subject-object or object-terrain - poetry can also be there and here here and here, in and in, in and out, now and then or vice versa, in any order. It is off-the path, yet it is the distantimmediate flicker guiding the way on the road. Poetry's relationship to vision is visionary, not visual - the poetic world is not a terrain, it is a feeling. Poetry's relationship to language is morphing and metamorphing, not physical and metaphysical - when a flower blooms it's not a description of anything, it just blooms in its beauty. Poetic language is not uncovering another layer of reality, it is channelling itself - it is not about consolidating a coherent linguistic universe, 
it is about shutting down language altogether; it is about silence. Poetry doesn't communicate something beyond itself and thus it can leap beyond language itself. The poetic journey doesn't need to cover distances to reach the end of its world, it is always already there - all it needs to do is switch language off by using language itself and the feeling of this world can instantly dissipate. Like the whimsical neutrino which is claimed to occasionally surpass the speed of light, thus crossing the boundaries of the physical into the imaginary of other dimensions, poetry hovers at the frontiers of the known.

But what is this poetry we are talking about? The word "poetry" comes from the Greek poiesis $(\pi \circ$ oínбıs), roughly translated as making, production or creation. Production not as a mechanical process but as gathering (Proto Indo-European) and bringing-forth (from $\pi$ oté $\omega$, "to make, bring forth"). It also refers to the final product of that bringing forth as that which has come into being. This double sense of poiesis as that which powers both the making and the made opens a view on manifesting explored by Heidegger and Maturana in their respective versions of poiesis as ecstatic bringing-forth (Heidegger 1977 [1960]) and of autopoiesis as the non-linear dynamics of life whereby the producer and the produced coincide in the same organism (Maturana 1980).

Techne $\left(\tau \varepsilon^{\prime} \chi \eta \eta\right)$, another ancient Greek word for making, means making as crafting, the how-to of the process, the skill that allows for human or divine production - the art of making. According to Plato, techne is applied in two types of production - the making of actual physical objects mimetic to the ideal Forms (that abide beyond the physical world and are imperfectly imitated by it) and the making of imitations of those physical objects such as the works of painters, for example. Thus the crafting of a chair was considered a more valuable work because it was closer to the ideal form (of a chair) than a painting of a chair which was twice removed from the domain of the ideal, the original so to speak. (Plato 2004 [380 BC]). Symbolic 
production was thus considered less authentic than material production and this mimetic alienation of the symbolic from "the truth" (Plato Ibid.) was seen as dangerous in its deceptive power. This vision of alienation by degrees by means of techne presaged the unfolding of traditional technologies into Benjamin's means of mechanical reproduction - a technology that can potentially carry out mimesis of mimesis ad infinitum.

While techne referred to the linear mode of producing, i.e. producing that which is similar, poiesis referred to dissimilar production, a more creative, originary, sense of bringing forth, such as the blooming of a flower out of a seed (natural poiesis) or the clash of clouds into a storm. Thus, techne was a kind of poiesis as far as making was a kind of bringing forth, poiesis could be a kind of techne as long as it was instrumental to a certain process of becoming. While poiesis could employ the energies of a body-form to bring forth a new form of that body, such as the growth of the embryo into a mature organism (as in Maturana's autopoiesis), techne was reliant on pre-set external forces, such as in the maintenance processes of an organism (food, air, cell maintenance and regeneration, etc.). In short, although poiesis was a kind of techne and techne - a kind of poiesis, poiesis was involved with generation and original production, while techne was the way of re-generation and mimetic production. As such techne was native to that which was to become modern mechanical reproduction.

However, Heidegger's view of techne intertwined it more intimately with poiesis in a way that would highlight a deep rift between traditional and modern technologies. It is in the ancient view of techne as a kind of poiesis that Heidegger finds the essence of technology - techne-aspoiesis (Heidegger, 1977 [1954]). This original sense of technology Heidegger based on the ancient Greek notions of techne and episteme as ways of knowing, ways to truth. Technology, according to Heidegger was revealing (truth) and enframing (the knowable), i.e. as a modality of knowing and manifesting: 
"Whoever builds a house or a ship or forges a sacrificial chalice reveals what is to be brought forth.” (Heidegger 1977 [1954], 13)

Thus, as a mode of revealing, techne was primarily concerned with bringing forth, not with making and producing, therefore it was essentially poetic (and originally aligned with the arts rather than the techno-science of modern technology). However, the procedural character of techne was that which outlined it from poiesis, not in terms of Heideggerian essence but in terms of practical irreducibility, and made possible the unfolding of traditional into industrial and postindustrial technology. Those two angles on technology, the procedural and the poetic, interpolate in ways not always linear and direct, but always threatening to shatter the manifold of human thought if not considered in concert.

Techne-as-poiesis promises a union of logic cognitive ways, a union both encoded in this more original sense of technology and obfuscated by a techno-scientific impulse to over-power through separation. Traditional technologies extend the body, as in the famous example of Heidegger's hammer, or in the painter's brush and the musician's harp. They are set in motion and powered by the energy of the body. This makes them energetically contingent on the artist, which is another way to say that Benjamin's aura is contingent on the energetic process that impresses itself on the art work. Modern technologies (mechanical reproduction) replace the body - the factory conveyor belt can work with minimal human input and so can the auto-recording camera or the sound recorder. This independence of modern technology from the human presence makes possible the exploration of hitherto inaccessible areas, such as distant planets (satellite scouts) or body organs in process (endoscopic camera) but at the same time disconnects technology shifting the energy investment from the native body to external host mechanisms. This enlarges the mapped world but disempowers the human body, even if only by the assumption it inspires that all power is purely mechanical (e.g. the importance and value imbued in prosthetic devices). 
Thus, modern technology has the peculiar capacity to penetrate the organism and the universe (the micro and macro) as it disconnects itself from the source of its initial impetus - the human form. The impulse to surpass the human domain and its limitations is embedded in the human condition itself insofar as language can be used as mental projectile. The passage of sensible experience through thought can then be projected further into the extended experience of the body through technology. However, while traditional technologies circuit back to the body-mind through the art/isan work, industrial technologies can be easily discontinued from the physis of the body and lock the human in the spatio-temporal coordinates of a meta-physical universe. The impulse to surpass the human domain extends into what Arendt identified as world, and subsequently earth, alienation. (Arendt 1998 [1958]) The loss of the poetic flicker as granted through the creative energies of the body may have ultimately meant an entrapment into a mappable universe where an impotent body-mind remained suspended at the mercy of a life-less technology promising to extend the process of life (Arendt, Ibid.) endlessly.

Thus poetry is not only a force complementary to reason, as the imagination-logic discussion might have implied, it is that which bridges the coming forth of logos into language, as it bridges non-being into being, it springs from the original creative force - according to the ancient Greek understanding of the word, as handed down to us by Plato:

"There is poetry, which, as you know, is complex; and manifold. All creation or passage of non-being into being is poetry or making, and the processes of all art are creative; and the masters of arts are all poets or makers." (Plato $360 \mathrm{BC}$ )

One way that poetry, as creative movement, shows itself to us is through the erotic impulse which, according to Plato, drives the fundamental human desire to transcend the temporal cycle. This drive flourishes within beauty as the "destiny of goodness." It begins with 
the simple drive to love one beautiful body, with the lust of the physical body unto pro-creation (natural poiesis):

“... and first, if he be guided by his instructor aright, to love one such form only-out of that he should create fair thoughts; and soon he will of himself perceive that the beauty of one form is akin to the beauty of another; and then if beauty of form in general is his pursuit, how foolish would he be not to recognize that the beauty in every form is and the same! And when he perceives this he will abate his violent love of the one, which he will despise and deem a small thing, and will become a lover of all beautiful forms; in the next stage he will consider that the beauty of the mind is more honourable than the beauty of the outward form. [...] ... and will search out and bring to the birth thoughts which may improve the young, until he is compelled to contemplate and see the beauty of institutions and laws...; and after laws and institutions he will go on to the sciences, that he may see their beauty, ...drawing towards and contemplating the vast sea of beauty, he will create many fair and noble thoughts and notions in boundless love of wisdom; ... and at last the vision is revealed to him of a single science [originally "episteme"], which is the science of beauty everywhere." (Plato 360 BC)

But this is only where the socially explicit, quest exhausts itself. Love drives the student from the experience of sensual beauty to the logical understanding (episteme as knowledge derived from logos as argument) that beauty is everywhere. But this understanding, similarly to the poet's quest, does not come to him by way of logical reasoning - rather logical reasoning is "revealed to him" as a response to his poetic acts of contemplation ("create many... thoughts"). Only in poetic thought the initiate exerts mental action, everything else is granted to him by way of this poetic effort. The metaphysical view of the world as one manifestation of beauty is a vision of a certain way to grasp cohesively the world of separations he started from. Finally, this path from sensual to epistemic knowledge leads to a threshold where the initiate may be granted an insight into the mystery of beauty, absolute beauty, seen as that which begets reality itself:

"But what if man had eyes to see the true beauty-the divine beauty, I mean, pure and dear and unalloyed, not clogged with the pollutions of mortality and all the colours and vanities of human life-thither looking, and holding converse with the true beauty simple and divine? Remember how in that communion only, beholding beauty with the eye of the mind, he will be enabled to bring forth, not images of beauty, but realities (for he has hold not of an image 
but of a reality), and bringing forth and nourishing true virtue to become the friend of God and be immortal, if mortal man may." (Plato, Ibid.)

"Not images of beauty, but realities" - the mental movies so freely played out in dream, where from the quest of Stefan George's poet begins, if upheld by the communion that the quest affords, may open into entire realities, into a domain where physicality (the beginning of the erotic initiation into the truth of beauty) reveals itself as but one modality of being among many. The poet's journey begins somewhere in the middle of Diotima's ladder - the poet, unlike the biologist, is not concerned with the natural poiesis, his quest is in the domain of language itself, the domain of thought and poetry. This is why he starts with travelling to the edge of thought and experience - the domain of dream and wonder:

"Wonder or dream from distant land

I carried to my country's stand

And waited till the twilit norn

Had found the name within her bourn -

The I could grasp it close and strong

It blooms and shines now the front along." (Heidegger 1982 [1971], 60)

The poet searches for the strange and the unusual, for that which is barely known, but still within the bounds of the known, and brings it into the familiar, into his "country's stand" which, for the poet, is language:

"Wonders and dreams on the one hand, and on the other hand the names by which they are grasped, and the two fused - thus poetry came about." (Heidegger 1982 [1971], 68)

And this is only the beginning of poetry - the movement of bringing forth the logos in the flesh, of calling out the thingness of things by naming them, is mimicked in the poet's individual path as the impulse to bring the strange into the familiar, the spoken into the unspoken:

"But when the issue is to put into language something that has never yet been spoken... Such is the case of the poet. Indeed, a poet might even come to the point where he is compelled... to put into language the experience he undergoes with language." (Ibid.) 
Which is what Stefen George does - granting us a view into his ways and insights. What we witness is his linear quest in the forest of the world, of the known-as-knowable, that which has been already granted us by logos as language, but not yet fully explored - he searches as a traveller, he explores the far corners of language for that which has not yet been spoken, but is speakable nonetheless. He is no longer the art student, mastering the mimesis of form, now he is fluent in it, and ventures to explore its domain with the courage of a pioneer. The love of one beautiful thing, the domain he's mastered, "his country's stand" - language - allows him to find the "beautiful everywhere" and to bring it back, to enlarge that domain, in a way. But this (still linear) quest brings him face to face with finitude. Finally, the poet arrives at a place where no mastery or metaphysics can help him hold onto the known anymore - he is brought face to face with the essence of language itself. "He obtains entrance into the relation of word to thing." (66). And not by rhyme or reason, not by imagination or calculation, not by art or science - he lets go of all this and experiences an in-sight:

"So I renounce and sadly see:

Where word breaks off no thing may be."

This insight is not a way to acquire "mere knowledge" (66), it grants him the wisdom of beholding the staggering view of how the reality of his world is sustained. Pursuing the strange and unfamiliar, the wonder and dream, had expanded his boundaries and had prepared him for the encounter his path was leading to - the encounter with the unknown, that which lies beyond existence:

"What, then, does the poetic experience with the word show as our thinking pursues it? [...] It shows what is there and yet "is" not. (87)

In the forest of language, poetry showed itself as "manifold." The poet had to search far and wide to bring it together - a quest into the domain of discernment and separation that 
metaphysics affords. The domain where concept and object are matched but not tied, where their correspondence is important so that the poet may "grasp" them and allow them to shine forth in his work, but is not yet privy of their existential relationship. What the poet is still working with are but projectiles of word and thing, of that which sustains the world. They are necessary simplifications which make possible the entrance and travel into the strange world we are thrown in as cognate points-of-feeling that are to become lovers, artists and scientists on the way to death. Only when the poet reaches the end of his world, only at the edge of the precipice, the Nietzschean abyss, when he sees that the word sustains the thing, does he really step beyond metaphysics. Similarly, the ancient Greek initiate has to travel through all the countries of worldly experience, from erotic procreation, through politics, the arts and sciences, to finally gain access into a realm beyond description where realities themselves are begotten. This is where any well trodden path leads to - to the path that cannot be trodden, to the way. And this is the only actual duality - of realms that beget each other - the poet or philosopher can hope to be granted access to. Tao Te Ching, Lao Tse's work alluded to by Heidegger throughout "On the Way to Language" begins thus:

"The Tao that can be trodden is not the enduring and unchanging Tao. The name that can be named is not the enduring and unchanging name.

(Conceived of as) having no name, it is the Originator of heaven and earth; (conceived of as) having a name, it is the Mother of all things." (Lao Tse in Müller 1891)

It is this primordial nameless silence that language talks about when we enter into a free dialogue with it:

"But when does language speak itself as language? Curiously enough, when we cannot find the right word for something that concerns us, carries us away, oppresses or encourages us. Then we leave unspoken what we have in mind and, without rightly giving it thought, undergo moments in which language itself has distantly and fleetingly touched us with its essential being." (Heidegger 1971, 59) 
The Mother of all things, logos, the realm of absolute Beauty, the holomovement, shine forth through language-form that, through the meandering paths of poetry and reason, may bring to that original domain the adamant traveller, now strong enough through the mastery of form, to behold the formless, the nameless. But it is not the first cause of science the traveller is allowed to behold, as cause is only a concept that belongs to the nauseating circuitry of metaphysics - the traveller is allowed to peep behind the curtain of death, behind the only certainty afforded the human mind. But isn't that gesture of peeping, of stepping behind the curtain of the known, a gesture of dying?

The striving for immortality Diotima sees as the beginning of the human quest into awareness, it is but the shadow of the human walking unto death. Passions of the flesh and mind that could, in their pleasurable immediacy, turn into fear and closure to the unknown making the student "clogged with the pollutions of mortality and all the colours and vanities of human lifethither looking" could also, in their immersive nowness, allow the courage to remain open to whatever may lie beyond the sensible realm, to the monstrous nothingness of the unknown. The striving of immortality, the drive behind the human quest into knowledge, passes through death itself and those who succumb to "the colours and vanities of human life," to life as avoidance of death, to life as an endless self-same process (Arendt) are the ones doomed to decay into death; the student who, in his striving for immortality, accepts the inevitable encounter with death, not trapped but propelled by mortal passions, may be granted a view of the abyss, a relationship to death as a gateway to the unknown and not as a guillotine of the known. Although both Heidegger's poet and Diotima's initiate have to master the known and, in doing so, exert themselves to the limit of their capacities, the final opening does not come from them, it is always granted. Even epistemic knowledge (position obtained through rational argument), the precursor 
of the scientific modality of knowing, is but a vision granted in contemplation, and not arrived at by linear mental effort :

"...and at last the vision is revealed to him of a single science [originally "episteme"], which is the science of beauty everywhere." (Plato 360 BC)

The phenomenological flickering of perception would therefore be not just, as Virilio would have it, a "piknoleptic" pathology (a systemic condition of consciousness lapsing) obfuscated by the rational cosmos of techno-scientific linearity (Virilio 2009), but also a poetic leak through the cracks of metaphysical reality. The vision revealed through those cracks is given to and not generated by the student. While the process of contemplation, a process of mindfulness in "drawing towards and contemplating the vast sea of beauty," is a private process in which the mind generates its works in a bid to "create many fair and noble thoughts and notions in boundless love of wisdom," this process is only a disciplining of the mind so that it can grasp that which does not come from it, the mystery granted by the unknown. In short, Diotima speaks of thinking as conjuring and wisdom as a matter of love, not an object of ownership. Thus the quest of the student is not merely an epistemological quest to acquiring knowledge, but a labour of love, a love of wisdom, it is philosophy.

"Thinking is not a means to gain knowledge. Thinking cuts furrows into the soul of beings." (Heidegger 1971, 70)

It is the way human sculpts time - not only a harsh, alien, cold gun for shooting out-ofbody projectiles, but also a hot, odorous force that made Nietzsche dream of summer in his call for fragrant thinking. (Ibid.) This passionate odorous thinking has the power to turn abstract questioning into a live scene with the stench of cow dung and grass that offers the solution to cosmic space-time conundrums, as in the famous dream of Einstein that allegedly inspired his theory of relativity. 
"The very reason we need logic at all is because most reasoning is not conscious at all. " (Jaynes 2000 [1976], 1)

Logic transposed onto dream arguably helped Einstein solve the riddle of the speed of light, conjuring an empirical scene impossible to render in the physical reality of wakefulness. This transposition could have been but a matter of attention - bringing the restrictive order of logic to dreaming and holding it there. Although difficult, when accomplished such transposition could stabilize dream images into a hyper reality that aligns the manifold of wakefulness and solves the multi-layered riddles it poses. That power of dream becomes a power of wakeful thought granted to Diotima's student, not unlike the power of the word to uphold things given at the end of the poet's journey:

"...in that communion only, beholding beauty with the eye of the mind, he will be enabled to bring forth, not images of beauty, but realities" (Plato $360 \mathrm{BC})$

A dream (i.e. thinking) unhinged, can behave as a moving target in wakeful memory, slipping the dreamer's attention, briefly emerging in consciousness and then quickly submerging in the dark matter of the unconscious, often graspable only in the liminal moments of waking. But when held by wakeful attention, disciplined by the presence of things, and even more so by the (metaphysical) resistance of objects, it can make the imaginable tangible, as in Einstein's calculations, or feed the poetic cinema of Deren and Dali. The imaginary world of Morpheus, dream, is the realm of thought, unconstrained by any outside rules or forces. Slowing down the wild flow of dream images is the beginning of thought - the experience of thought in wakefulness is but the disciplined flow of dream. When considering the matter closely, it is hard to identify any conclusive qualitative difference between dream and wakeful thought, except degree of control. When Einstein went to sleep and dreamt of cows answering his question about the speed of light, he didn't go into a mindless reverie, his answer didn't spring from an eccentric whimsy - 
he went to the source of thinking itself and this is where his answer was first granted. Both dream and thought are form fields that can shift and flow unrestricted by space-time.

But the wakeful control of thinking does not spring from the thinking subject. The discipline of thought, its relative fathomability, comes from the encounter of things, and not from thought itself. Yet thingness, the animate expanse of energy, is disciplined by the stabilizing hold of form, the gift of dream. Thus dream (or thought) as the stabilizer of thingness and things as the stabilizer of thought abide in one neighbourhood, as Heidegger would call it. Language as the stabilizer of thought (Elder) and the sustainer of things (Heidegger) is the way through which the reality of thought-things emerges, i.e. the way it comes into focus. Diotima's beauty is therefore the mysterious spirit that animates thingness and thought sustained by attention, by the "eye of the mind" - the focus of mindfulness - achieved through a lifetime of discipline through language, or, more specifically, through the stabilization language, as a system of organizing experience, affords.

The way of exercising visual attention is through focus but, interestingly, focus is not necessarily an optical term. The original Latin use of the word referred to "hearth" as fire and centre - as the warmth, energy and light that sustains the home. Hearth was the heart of the home. "Used in post-classical times for "fire" itself, taken by Kepler (1604) in a mathematical sense for "point of convergence," perhaps on analogy of the burning point of a lens," (http://www.etymonline.com/index.php?term=focus) focus is the assembling point of reality, that which can stabilize the fleeting phantom of a thought, a perception, a vision, a hallucination into a thing - a sustainable, explorable, mysterious being that does not crumble under the whims of the mind. As such, it is not a mere optical phenomenon - it is arguably the only freedom granted to the human being. It is not just an optical mechanism or a product of neuronal activity, it is also a poetic emergence - focus comes from the heart, the hearth, the energetic centre of the body. 
This libidinal dance between logic and imagination, between dream and wakefulness, between form and matter, is fuelled by the love for Diotima's student. It starts as the love of a single beautiful body and expands to love of all beauty. The all-encompassing beauty Diotima talks about cannot be a specific mathematical expression or personal preference. Its grant, which can be both extremely precise and entirely general, is bestowed on "everything" - it is therefore nothing short of the (formal) expression of a force that runs through every thing. It is the way the student can behold the unknown - love of beauty is a love of the unknown, i.e. a love of knowledge, of wisdom that is not about knowledge or wisdom. Beauty, for Diotima, is intimately tied to the mysterious - a view that, centuries later, has inspired Einstein to famously say:

"The most beautiful thing we can experience is the mysterious. It is the source of all true art and all science.“ (Patrick 1935, 44)

The mysterious as the unknown, reaches us in the leaks of poetry dripping through the membrane of reason. It is the glow of death from the edges of the known. Both the poet and the initiate, in their relentless quest, can only prepare themselves as openings for the eventual grant of Death as they, instead of letting death advance to them, advance toward it "renouncing" the world they have travelled, the very world that has afforded them the journey to the beloved mystery. There is neither image nor body, neither dream nor wakefulness, neither idea nor object where the "word breaks off" to unleash the impossibility of being:

"...if the word is to endow the thing with being, it too must be before anything is - thus it must inescapably be itself a thing." but according to the poet "Word and thing are different, even disparate." (Heidegger 1982 [1971], 86)

Thus the word and the thing, as sustaining the known, are together not causally, but mutually supportive, both propelled by a source of inarticulable mystery that holds nothing known, including the word that sustains things: 
"...the word can never be found in that place where fate provides the language that names and so endows all beings, so that they may be, radiant and flourishing in their being." (Ibid.)

The word is not found where the word is actually generated by language. That place, beyond the breaking point of word and thing, can be felt in the flicker behind the curtain of death, in the flicker of poetry. So, poetry can be viewed as the (necessarily) thinkable shadow of something that, although articulated through language into the realm of reason, insofar as it is knowable, is nonetheless not entirely native to language insofar (as it opens up language to the unknown). And yet, there is a sort of distillation of the essence of poetry that manifests in language, an arrival of the original impulse of poetry into human expression. Through the "manifold" fractures, fragments and wanderings of poetry into being, its singularity shines forth in language, as in Socrates's continuing conversation with Diotima:

"Still," she said, "you know that they are not called poets, but have other names; only that portion of the art which is separated off from the rest, and is concerned with music and metre, is termed poetry, and they who possess poetry in this sense of the word are called poets." (Plato 360 $\mathrm{BC})$

Music pertains to "the techne of the Muses," it is that which links human making to the realm of the unknown, which opens the human to receive insight or inspiration by the Muses, who are not human and yet, thanks to poetry, not beyond human grasp. Poetry, by mobilizing both the imaginative (music) and the logical-mathematical (metre) faculties of the human, therefore makes manifest the knowable. This intertwining of human making and non-human creation in poetry is what Heidegger probably alludes to when he explicitly evades poetry's location. "Poetry moves in the element of saying, and so does thinking. [...] We cannot here decide flatly whether poetry is really a kind of thinking, or thinking really a kind of poetry." (Heidegger 1982 [1971], 83) 
The pendulum of poetry-saying-thinking swings language toward the sea of becoming, which encompasses but does not remain in the Said, the Thought, the Poem, the accomplished. "To say means to show, to make appear, the lighting-concealing-releasing offer of the hold." (107)

In other words, saying as "the being of language" (Ibid.) both makes apparent and conceals itself and, in doing so, in enframing both the revealed and the concealed, the known and the unknown, saying releases us. The liberating power of saying is in its offering of in-sight ("lighting") rather than a conviction, of an unbound movement in the space between mystery and manifestation rather than a commitment to either. The saying is what the poet was granted entrance to at the end of his journey. "The word avows itself to the poet as that which holds and sustains a thing in its being" (65-66) and by doing so, offers the poet a glimpse into wordless silence where "no thing may be."

But is this realm of silence a realm of non-being or a realm of Being beyond thingness and even beyond logos? If this question were posed in the domain of conceptual language, the language that scans and packs reality into a self-contained symbolic system of relationships, the answer would be the former - the poet has reached nothingness, as there could be no-thing beyond thingness, and because being, from within language, is the being of things that language sustains. But Heidegger informs us that at the end of the poet's journey, the prize does not "crumble into a nothing that is good for nothing. The word does not sink into a flat inability to say. The poet does not abdicate the word. It is true, the prize does withdraw into the mysterious wonder that makes us wonder." (88) So, if the question is being posed from the domain of language as language, its end opens up to a no-thing pregnant with the mystery that poetry, as that which permeated language all along, has been intimating. The mystery of being that infuses thingness and language but is not infused by it. 
And let us leave that mystery untouched by the musings of the mind as those will only make it recede into obscurity even more. Instead, let's leave it linger ever so closely, as we turn away and focus on the more intellectually malleable question of how poetry permeates language. According to Heidegger, poetry is either a kind of thinking or thinking is a kind of poetry, while at the same time thinking is what we are given in order to listen to (and hear) the Saying granted through language. In other words, as thinking is a way to hear language, poetry is therefore related to the unfoldment of language, if it is not the unfoldment of language itself. The manifold language-thinking-poetry is thus neither causal nor consecutive; it is rather a triad that works in unison to keep an opening to meaning as Saying.

To Diotima, poetry is the techne of the Muses (that gives us music), restrained by metre. It seems that here too, once again, Poetry is then both a kind of thinking and thinking a kind of Poetry - the (breaths of the) Muses enter the human domain through thinking and linger in music and rhythm as kinds of thinking. If language is listening, then we listen to Poetry as the techne of the Muses through our thinking. Therefore, it could be said that thinking is the language of Poetry: when, having listened to the Muses, we channel forth the Saying in a humanly crafted poem, we exercise poetry as the language of thinking.

But when does thinking reveal itself as the language of Poetry? It would be where thinking reveals itself as thinking, where thinking is alone with itself, moving toward itself. We find thinking only in its absence. But thinking is with us all day long, it is part of the contemplative pause and the busy day, of lifting this cup or solving a complex problem, when we think smart things and stupid things, even when we think we are not thinking. And when we try to stop it, thinking ambushes us, revealing itself as thinking, beyond empirical restraints and wilful considerations. As Nietzsche observed, thinking happens to us (Nietzsche 2003 [1886]) so, when thinking finally withdraws, in that moment of inner silence when we are finally alone, we also 
withdraw from thinking. And then, in our sleep or slumber, thinking unleashes as thinking. Free to be as it comes, thinking reveals itself in dreaming.

What do we see in dreaming? We see a flood of formal possibilities - what form can do if unhindered by mass, by empirical restraints, by space-time. In dreams, things defy gravity and logic, events happen simultaneously, bodies occupy both left and right, up and down, identities merge and multiply. Dream is an orgy of poetic visions - the gateway of the Muses, the house of thinking. Therefore, as long as thinking is thought of as a condition of wakefulness, there is more than Husserlian intersubjectivity to being in the world - we dream each other in wakefulness, we dream ourselves into being. There is no metaphorical or even any mystical connotation to this statement - it describes a being with thinking in a way that at once validates and vaporizes the Cartesian position. Validates in as far as it establishes a vital relationship between the domain of thinking and that of being, and vapours because the thinking it places alongside being is neither causative to the latter, nor has much to do with the subjective thinking Descartes had in mind (despite the fact that dream is the most subjective experience). Thinking has little to do with the subjective processing of thoughts, just like the language of Heidegger (the language that sustains the world in its "isness") has little to do with correct wording and more with the moments when "we cannot find the right word for something" and leave it unspoken.

When thought does not interfere, language touches us with its being. Conversely, the inner silence, the withdrawal of language from thought, unleashes a being-in-dreaming devoid of things - a wordless field where images are not sustained by the agglutinating force of language.

And yet, we do speak in dreaming; indeed, the images of dream unfold as language. In fact, we often speak and hear more fluently in dream than in wakefulness. It could be said that, in a way, dreaming is where language, not just thinking, is given free reign. Language in dream floats freely, unfettered by references, things, and above all by its signifying function - a word in 
dream resonates in itself. Moreover, all dream forms resonate in their unfettered singularity - they do not signify or convey codified meaning, they infuse meaning by touching our mind directly, the way the sun touches our skin in wakefulness:

I dream that I am in my house, but the place has nothing to do with my house and yet I "know" beyond a shadow of a doubt that this is my house. I step in, I am someone else, a man wearing a grey suit - I know myself as a man wearing a grey suit just as clearly as I know myself as the silent formless witness of the movements of this man in the dream scape. We are both there knowing what we know, without any need to explain, justify or prove that knowledge. It is not contingent on any formal movement, it is beyond language. And yet, it is precisely the fantastic flow of form, the bleeding of language through the cracks of reality opened by dream that affords this knowingness. The only constant in that picture is the nameless awareness of the dream witness, the point of view I had been reduced to - an awareness without attachments, beyond time-space, beyond continuity, a witness of the flow of nowness.

The dream witness pays attention without paying attention. It is anchored in its formless self because every time it attempts to anchor itself somewhere in a form, every time it attempts to focus on any specific item, the latter dissipates or morphs into another. There is no here-there in dream, no stable identity and no distances -the realm of dream is spaceless. Thus the dream witness has no identity/boundaries, it lets itself become the dream itself. By contrast, wakeful awareness is anchored by specific things - the more it attends to/focuses on a specific thing, the more stable it becomes. Thus the wakeful mind is grounded in the specific presence of things, which, with the advent of language, crystallizes into (formal) identities and subsequently, becomes itself embodied by an identity. Through the presence of things, the spacelessness of thought as dreaming awareness becomes wakeful awareness. Through the advent of language, wakeful awareness becomes consciousness in the Jamesian sense, as linguistic meta-cognition: 
"...consciousness is an operation rather than a thing, a repository, or a function. It operates by way of analogy, by way of constructing an analog space with an analog ' $I$ ' that can observe that space, and move metaphorically in it. It operates on any reactivity, excerpts relevant aspects, narratizes and conciliates them together in a metaphorical space where such meanings can be manipulated like things in space.” (Jaynes 2000 [1976], 66)

Could it be that the destination of language is therefore consciousness itself, the discipline of being into consciousness? And if so, wouldn't that mean that, given the interpolation of language and being that would imply the disciplining of consciousness into being? A twofold movement of energy and awareness that not only casts the projectile development of physical reality into conceptual language but also the concurrent sensualization of thought through poetic language and, ultimately, dream. But where do we experience those cross-pollinating forces of awareness? It seems we do that both when we bring wakeful attention to dreaming, as in Einstein's dream (or in the practices of lucid dreaming in general) and when we bring dreaming attention to wakefulness, as we do in the practice of ritual and art. Dreaming attention emerges in wakefulness whenever we abandon ourselves to the strange world of an artwork - a work built on the poetic trophies snatched from dreamland and superimposed on wakeful reality.

But wasn't art a mere mimesis, an imitation of reality? And couldn't dream be just the screen where imprints of our wakeful experience, of being as sustained by language, play out, like broken projections that have lost orientation? It could be all of the above: indeed, dream does become possible through embodiment, its dramas un-image-inable without sensual memories. Doesn't the odourless dream scape of thinking tend to disappear all too easily at the smell of morning coffee? And yet, dream is the gateway to the Muses, the house of thinking, built by experiencing the thingness of things sustained by language, a language which, as a kind of Poetry, enters through... dream. This Moebius circuit affords a conceptual sequencing that points a way for Heidegger's understanding of language as the House of Being to enter the same room with 
Metzinger's ideas of reality as indistinguishable from subjective neural processing (i.e. Of dream and wakefulness being the same projections on the concentric screen of the "ego-tunnel") (Metzinger 2009) without making the former seem obscure or the latter, simplistic. Moreover, it realigns thinking with reality in a way that allows to rephrase the question of mimesis - isn't art, before an imitation of reality, inevitably a mimicry of thinking? Thinking as the language of poetry is therefore most congruently realized, it is most authentic, in poetry as the language of thought, the way of art.

But, metaphysically speaking, where does this discussion position poetry as language? Poetry as a kind of thinking is also a way of knowing in so far as thinking is the way of meaning, and meaning - a way of organizing information into knowledge. Poetry, organized by the singular (witnessing) point-of-view, is native to dream; reason, organized by general (objective) principles of continuity, is native to the physical world. Both poetry and reason offer ways of knowing through language (language both as a sustainer of the world and a free-flowing complicity of forms; or language as system of referring and as formal system). The interpolation of those two modalities of knowing allows for the pursuit of the (specifically human) symbolic knowledge described in the current Occidental epistemological paradigm by the divide between the arts and the sciences (in accordance to the foregrounding of poetic or rational approach respectively). In language, a poetic form is necessarily encased in syntax, a rational structure (as far as the rational, as previously discussed, is concerned with isolation and organization of patterns and relationships). Conversely, the rational in language is necessarily encased in the poetic in so far as language as an arbitrary free-form symbolic system is a form of Poetry. Likewise, the dissolution of the rational into the poetry of dream is counterbalanced by physical autopoiesis sensually emerging and rationally graspable in wakefulness. Poetic and rational 
thinking as ways of knowing through language therefore appear within the larger field of that which is thinkable.

Since Greek antiquity, poetry and reason have been increasingly streamlined into the ways of art and science: art, concerned with the sensual manifestation of things, with formal apprehension, while science, with the mechanical manifestation of bodies, with how they move. Art has thus been necessarily concerned with Language itself, with language as a kind of poetry conjuring the world, science has been using language as a signifying instrument mapping the universe that includes language itself as an object:

"...scientific and philosophical information about language is one thing; an experience we undergo with language is another." (Heidegger 1982 [1971], 59)

The poet-artist is immersed in language the way a swimmer is immersed in a river, floating, flowing or fighting with it. The scientist, on the other hand, is sitting on the shore thinking about where the river might be going, as a fragment of it wriggles there, framed, pinned and bled by the distance.

"In experiences which we undergo with language, language itself brings itself to language." (Ibid.)

It reveals itself, the way the river would reveal its source to the swimmer abandoning herself to the flow. The scientist, on the other hand, can only observe the flow and draw conclusions about its behaviour. Poetic language, akin to Heideggerian traditional technologies, is revealing; scientific language, congruent with Heideggerian new technologies, is extracting. (Heidegger 1977 [1954]) Language as a means to an end, is therefore a kind of natural technology that provides ways of making.

But how can poetry be both the giver of language and a kind of technology working through language? And, if language is a kind of poetry, i.e. given by poetry, doesn't that imply 
that it has also autopoietically emerged as a natural phenomenon, uniting Maturana's (1972) with Logan's (2007) ideas of language as an organism? And then, if language is a kind of organism, how can it be also a kind of technology? Indeed, it is possible to think of and use an organism as a tool (e.g. a human shield), but the human body is before else an organism that can be used as a tool, not a tool that happens to be an organism. However, if we regard an organism as an autopoietic phenomenon, that is both independent and metabolically contingent on the environment, it could be said that an organism is a medium of both metabolic flow and of poetic morphology, as a non-linear, dissimilar formal unfoldment (e.g. from seed to flower). Thus organisms could be viewed as a form of natural technology that is primarily mediumatic and eventually, tool-like. In addition, a body, as a system emerging within an environment does so before all, as a tool of that environment - that environment supports its emergence only when it is congruent with the environment's dynamic state, or destination. Conversely, language, either viewed as an organism or as a kind of Poetry, is mediumatic when talked of as channelling Poetry into the world, and therefore a means to an end, a technology. As an exclusively human technology, symbolic language is mediumatic in a way pertaining to the human condition.

Thus, to realign Elder's statement that "words belong to the mind itself, and they are incommensurate with anything outside language" with language as that which disciplines thought in a structured sequential manner akin to the discipline of physicality, we have to regard "mind" as not merely the mental, but as that which, for the specific point-of-view includes the physical too in consolidating itself as that which represents the world, indeed is the world - that which allows the shapes of the world to emerge and solidify from the vibrational expanse that the pointof-view assembles or channels, and significantly, that which allows the syntax of language to sharpen the intelligibility of the phenomenal world, i.e. that which mediates the two. In that sense, the prioritizing of the mental, the physical or language can only be a matter of conditional 
ordering as they belong to the same fabric, the mind-world and, in disciplining and channelling each other, they interweave rather than cause and precede one another. Conversely, in this intertwined, co-supportive resonant autonomy (not only from the extramental (Elder) but also from the mental), language upholds its own reality. It steps into its deceptive (illusionary) potential only when it begins to conceptualize, when it begins to abstract itself from the thingness of things, signifying concepts rather than orienting toward things.

But this conceptual side of language is not an-other of language, it is not an opposing shadowy rational side that can be battled with raw grunts or poetic directness. It reveals the very destination of language as a conduit of consciousness, encoded in the very significatory potential of language. The dis-agglutinating power of language-consciousness, the power to make explicit the identifying form of a thing as separate from the thing, to separate form and matter so to speak, affords an articulation of the world that can accommodate the supra-spatio-temporal principles of thought/dream into the specific space-time of physical reality. This generates the spatio-temporal thinking that has been most meticulously explored in Physics, and which allows for the disentanglement from the embeddedness of the specific point-of-view, e.g. a conceptually adept human can imagine different solutions of a situation of entrapment, and eventually act to escape the situation (unlike a rabbit who can only rely on immediate instinctive bodily reactions). In other words, metaphysical language affords a degree of freedom that has a death-defying promise.

But this degree of freedom comes at a price. Truth becomes a central issue from the very onset of the age of reason, because the threat of losing alignment between Heideggerian language and thingness looms large way before the threat of confusing correspondences enters the Cartesian awareness. The very free-form character of language, its capacity to hover away from the thingness of things, to correspond rather than uphold, becomes the (shifting) ground of deception. When a sign is used instead of listened to, not seen as the flicker or whisper that 
orients toward a field but used as a label covering a thing, it becomes extremely mobile in its arbitrariness. It ushers the concept of representation which foregrounds correctness as the way to truth (correct correspondences between representation and presentation) and is itself represented in the visual arts trajectory from the Ancient Greek idea of mimesis (and its techniques) to the reproduction capacities of photographic technology (mechanical and digital). Representation displaces presentation already in the Platonic foregrounding of the mimetic aspect of art. For millennia before that, an Ancient Egyptian sculpture of a pharaoh was that pharaoh insofar as it provided a resting material place for his life-force $k a$ (Oakes 2003), not a representation but a presentation of his material life form, a receptacle and a channel of the same idiosyncratic configuration of energies that contained his live version. This trust in the power of form to channel energy and sustain being began to wither away with the conceptual breakdown of cognitive categories in Ancient Greece. This gave new powers to conceptual language which also activated new dangers. Language as a system of correspondences meant a free-floating collection of symbols in arbitrary relationships to matter. Such language might or might not adhere to principles of correctness, it might or might not follow rules of integrity because it has nothing to answer to but itself. Moreover, even when it does follow all those rules and principles, it cannot ever perfectly map the reality it claims to reflect. So, anything constructed by a language that claims correspondences, is deceptive, to a degree - from a specific conscious lie to a culturally constructed worldview that necessarily prioritizes certain syntactic positions over others (and has inspired Nietzsche's perspectivism as a suggested solution of this inevitable deceptive entanglement of language).

Thus language is authentically in its being when it embraces its deceptive nature, when it claims no correct correspondences. "Art is the lie that tells the truth," said Picasso and inadvertently pointed to the place where language abides. Art as the social shack of poetry, is 
concerned with language itself and not with its correspondences. Art is concerned with beauty as the truth of form. Beauty, the way of poetic language, doesn't convince, argue or prove - it shocks and thus shifts the awareness of Diotima's student, taking him or her directly to the edge of the known. Yet, to arrive there the student had to discipline the mind by journeying through layers of correspondences. Like the art student who has to learn first to master the mimetic powers of the pencil - the principles of proportion, light and shadow, angle, and the optics of perspective - in order to become proficient enough to use that same language of correspondences to invoke dislocation, destabilization and defamiliarization that wouldn't collapse into weak links and nonsenses but would instead fortify language by surpassing it, to use it as a trampoline to launch beyond it. This jump beyond language is not an escape from or denial of language - it brings back to language, to the mysterious nature of the thing that the word upholds, to the miracle of being. Thus the projectile nature of meta-physical language and conceptual thought, and respectively, of Jamesanian consciousness, discipline the mind in order for the latter to be able to withstand the blow of poetic destabilization as it brings back awareness to the thingness of things, to their unfathomable presence. The other alternative, the endless projection of conceptual thought into open space dooms the mind to the extra-terrestrial disconnect Arendt cautions about (Arendt 1998 [1958]); its flattening and sealing effect on reality (as a logico-linguistic loop) leads to a solipsistic view of existence and respectively, an ouroboric performance of life as a knowable utilitarian project of a community of subject-objects imprisoned by the cycle of use (and inevitably, through the magnification of progress, ab-use). The point-of-view-turned-subject, the only stable point of Cartesian reference, of certainty that is, becomes the anchor of a reality that, through its objective signature, constantly antagonizes the inflated ego-will by offering variable rates of resistances. This inevitably leads to a paranoid-schizophrenic Cartesian breakdown 
inaugurating the psycho-social dynamic explored at length by Deleuze and Quattari (1983 [1972]).

Thus language, as the distancing and connecting way of being in the world, as the symbolic expression - synthetic, syncretic, weightless and arbitrary - can also be called the medium par excellence - that which coincides with its form so thoroughly that McLuhan's "medium is the message" becomes shiningly poignant and completely redundant all at once. Conversely, language as the discipline and the way of the mind (as the world) becomes a medium of itself, the way of the mind toward itself, the way the mind finds and converses with itself, but above all, the way the mind becomes mind-full of itself (as the world). If those two aspects of language are severed, they tend to compensate for each other and thus arrive at nonsense not as a threshold to the pregnant nothingness of Heidegger, but as a collapse of language into the barren vacuum of mechanics - distancing language, working through disconnect, becomes solipsistic (Wittgenstein) and self-reflective language, working through looping, becomes disconnected. Thus we arrive at the two ends of insanity - the depressed scientist locked in the ouroboric world of reason and the raving mad man, lost in the wilderness of poetry.

As a medium, language is necessarily a means to an end - indeed, as a free-form way to both itself and the very thingness of things, it could be said that language is the original technology. The very first use of an Oldowan tool can be thus viewed as the inception of distancing through media-tion, the first linguistic gesture. This manifold interpolation of language, media and technology upholds symbolic communication as a way of making, just as poetry, language and reason uphold symbolic being as a way of knowing. Language, media, poetry and technology weave the manifold of symbolic being in the world.

"In experiences which we undergo with language, language itself brings itself to language." (Heidegger 1982 [1971], p.59) 
Thus poetry and reason, the two ways of being with language as technology, necessarily drive a medium, any medium, to its destination.

Cinema, as animated photography, has realized, to a hallucinatory degree, the idea of art as mimesis, first foregrounded by Plato. A 3-D cinematic viewing of a voyage, for instance, may induce somatic experiences so vivid that they can stand as convincing substitutes for an actual voyage. Art as mimesis of reality, a theme that has permeated cinema discourse since its inception, has achieved unimaginable by Plato definition - however, it could have never achieved it were it not, before all, a mimesis of the mind as the mental. Reality, that which has been stabilized by language as the interface between the point-of-view and the energies it converges, is the mind-world that emerges from that interaction. Therefore, reality is contingent on the mind as the screen of its stabilization. Prioritizing the notion of reality not only in film theory but, above all, in film practice, enacts a linguistic reversal that obfuscates the ways cinema (as art) acquires its hallucinatory power. Cinematic photography has stood as a hyper-rational evidence of an objective reality, that can become captive of modern technology as a free agent of rational knowhow, and can thus confirm the accuracy of the perceptual apparatus vis a vis its object out there. But, as we already discussed, reason as a modality of language does not map reality as much as it orients in the mind-world. Its descriptive power, as a symbolic mode which works by disconnecting, belongs to the mind (as world) and can therefore help figure out the workings of the world as mind, not a reality beyond the subject. The subject-object axis is a linguistic construct of descriptive convenience, not a reality anchor. Reason can only think reality when it arrives at the edges of the mind-world. Therefore, realistic cinema as mimesis of the mind-world, is an evidence of how we orient ourselves in that world - its power comes from its technical capacity to emulate the full spectrum of the rational way of knowing - from sensory perception to sequential extrapolation. 
Behind the notion of cinema as reality, lagging in a metaphorical cloud, is the notion of cinema as dream. That notion, however feeble and figurative, hints at the primary mimetic powers of cinema. If dream, as previously discussed, is the domain of thought unencumbered by empirical encounters - the extreme end of the mind-world as it opens up to the flow of poetry as language - then cinema is the closest human artifice has come to mimicking the domain of freeflowing image-forms, unconstrained by the syntax of embodiment. In other words, cinema as mimesis of thought is unique and unsurpassed by any artifice. Interestingly, dream as the supraempirical domain of thought has an irrational syntax, just as reality as the domain of physical encounters, of empiricism that is, is rational. This suggests, once again, that the dualities of reason, such as the counter-positioning of rational and empirical approaches, are but constructs of descriptive convenience. Art as mimesis of thought is most explicit in non-photographic cinema, such as animation and direct-to-film techniques. Yet those techniques require the highest physical involvement in the process of making - the poetic dream-like vision is derived from empirical encounter, from the raw touch of matter, not unlike the Apollonian visions of the oracle in Delphi were only given through the physical grounding of the Oracle in the womb of the Earth - a cave.

Reason and poetry as the two linguistic modalities of being in the mind-world are technologically explicated in cinema as mimesis of reality and cinema as mimesis of thought (dream). When do those two modalities unravel in cinema and how does cinema open up and restrict their flow? And, what is the destination of those linguistic movements, if indeed such a horizon is foreseeable? 


\section{Chapter 2. Method}

\section{The Body-Lingual Manifold}

Thus far, a simple, and far from novel, taxonomy of form (as channelling energy) has discreetly outlined itself: the elementary particle formation that agglomerates as substance, layered by the formal consolidation of matter into things/bodies, both blanketed by linguistic articulation. All of these modalities of form are (humanly) accessible as such through mediumship, which reorders the taxonomy thus: substances and bodies as semantically accessible through sensory mediumship and vice-versa (hence, the primacy of perception (Merleau-Ponty) in the conventional physical order of things), sensory mediumship semantically accessible through language and vice-versa (hence, the biological foregrounding of language emergence by Maturana and Logan e.g.) and finally, elementary particles (and cosmic organization alike) as accessible through the distancing/conceptual capacity of language (maturing in the scientific outlook).

But how could elementary particles become available to us through the mediumship of language when it is common knowledge that they become available to us through the mediumship of technology? Moreover, language is arguably a post-technological emergence, e.g. tool use is widely practised in proto-linguistic apes, both historically (in the evolution of the species) and concurrently (in the proto-linguistic apes that exist concurrently with Homo Sapience). If we depart from the most general definition of technology, as a means to an end, then, strictlyspeaking, not just apes but a range of proto-linguistic non-hominid animals use tools and strategies as technology. In the range of non-human technologies, from crows crafting and using hooks to fish worms out of tree trunks to octopus constricting shelters made of empty shells, 
proto-linguistic technologies bear all distinctions of technology that transpired in the discussion in Chapter 1 - they are distancing, protecting and violating.

How can then language be the condition of something that is already in full practice when language presumably emerges? If we consider it closely, language acts as agent of technology and vice versa (technology as agent of language) by doing what language does as language. As already discussed, language orients (movement) and focuses (things). But this coincides with what the body does as medium. And the body as medium is the condition of proto-linguistic technologies as they are all direct extensions and/or products of the body (from the grass blade a chimp uses to fish termites to the spider web). However, the body mediates through sensing, i.e. through contact (nearness) with the environment. It is the word, the symbolic formation of arbitrary sounds that can hover over things and directions, that affords (the mind) distancing from things to unify them into concepts. Thus, language breaks off as language when it begins to synthesize, i.e. where it becomes distancing-connecting, rather than unifying-separating as is the predicament of the body-lingual ape. When language meets the primitive technology of the hookusing crow, it not only identifies it as a specific technology, i.e. as a means to get worms, it also recognizes it as a mediumatic principle that is transferable to other situations where a resource is hidden (e.g. crow extracting food from containers by shaping wire in lab conditions). It is therefore the mediumatic specificity of language as abstracting-synthesizing that allows it to identify (distance) the mediumatic potential of primitive technology and to convert it (synthesize/connect) to a distancing-connecting, i.e. into linguistic tool. But this prioritizing of language and proto-linguistic technology is used here only as a convenient manner of speaking it would be more precise to say that the human language and human technology appear as outgrowths of a branching of the body-lingual medium. 
The distancing-connecting way of language already hints at conceptualization conceptualization as the destination of the distancing-connecting way of language. It is potentially already an inception in proto-linguistic tool use - the identification of a body as an object that can be manipulated independently of its immediate environment - and becomes an immediate possibility with the first generalization. We now know that proto-linguistic animals like the Caledonian crows (Wimpenny, Weir, Kacelnik 2011 [2010]) can generalize visually. This provides empirical support to the view that language principles precede linguistic development in the very structuring of reality in sensory experience, i.e. the view of a body-lingual being that branches off into language and technology as independent of the human body in Homo Sapience

While a word can be a name sustaining a particular thing in its unique presence in the formal mesh, it becomes a building block of language only when it distances itself from the particular thing and becomes a free floating attractor of all things that share those qualities which isolate the thing in the sensory expanse. (This sequencing is only a manner of speaking, it is not meant to prioritize the emergence of word over concept, i.e. to use conceptual gradation, except for convenience.) The pre-linguistic child who avoids bumping into hard objects has already isolated a rule that brings phenomena together as hard/impenetrable and thus has forged the condition for linguistic conceptualization. By branching off from phenomena, or conversely, entering the phenomenal world through its morphological character, the distancing-connecting way of language forges a protective layer over the phenomenal world. By protecting, this layering not only conceals but also signals (and potentially reveals) what is being hidden or protected, just as the anthill or the octopus shelter signal the possible presence of their inhabitants. Thus the proto-linguistic non-human technologies of over-layering, such as beehives, nests and other forms of sheltering, make explicit another way of distancing-connecting pertinent to language as structure: by restructuring the environment, bees, birds, ants reconnect with it in a new way that 
both protects them and, by signalling their presence, makes them vulnerable. It is important to note that layering itself as distancing-connecting is not conditioned by conceptualization - this is why, although structuring might appear as contingent on conceptualization, just as the wordthing, it emerges in the phenomenal world. Therefore, layering, although by definition distancing, nonetheless is not necessarily conceptual. Interestingly, the first human Biblical technology, the fig leaf, is both layering (the body) and conceptual (positioning/discriminating the body). The identification of the point-of-view body as vulnerable in relation to its environment is coined as the beginning of conceptual, and by extension, linguistic, thought. The use of a tool (the fig leaf) as a layering device (clothing) and not as an extracting/aggressing one (as in crow hooks, grass blades, etc.) is foregrounded as primary to the beginning of human culture. (1)

In converging word, structure and concept the layering character of language brings together the three ways of primitive (non-human) technology - extraction as un-layering (hooks, fishing blades), structuring as over-layering (anthills, nests) and insertion as in-layering (spider webs, beaver dams). Spider webs and beaver dams neither protect from nor violate the environment - instead, they interrupt it, by inserting an artifact in the flow of the natural continuum, in order to redirect and ultimately harness its energy. Technological interruption is a way of distancing-connecting by in-layering (inserting a layer by rearranging the pathways of energetic flow).

Thus from the non-human inception of technology as a means to an end, the latter makes possible three things vis a vis the user's body-mind - to extend it (tools, un-layering), to cover it (shelters, over-layering) and to stand for it (traps, in-layering). Vis a vis other body-minds, it reveals (tools), signals (sheltering) and traps (traps, webs). Thus the original medium, the pointof-view or body-mind, which as medium is channelling (the environment), by extending itself through various forms of technological layering, opens up the potential for constructing the 
environment. This becomes fully possible with the emergence of language which, by standing for phenomena in evacuating the signal from its physical presence, allows for a comprehensive layering through a description blanket that stabilizes sensory description/interpretation. Linguistic projecting of phenomena makes this blanket independently manageable, i.e. contingent on its own, physically unbound, set of rules.

The split of primitive non-human technologies into human language on one hand, and human technology on the other, made possible the layering of interpretations and technologies that would not only carve out and distance things through concepts but also through different conceptual syntax. The emergence of human language afforded an abstraction from physical restraints and the emergence of a disembodied point-of-view that pulled technology out of the gravitational field of the body and the body out of the gravitational field of the mind. Thus the crow's hook was no longer confined to being a mere tool, i.e. an extension of a necessary body. It could also become a free object used to accelerate, project and enhance the work of the body. The body-lingual non-human ape would use a sharpened stick not only as hook to uncover the hidden but also as spear to more effectively attack and kill the direct opponent/prey, but only the movinginto-language hominid would throw the sharpened stick as spear precisely and directionally, thus letting it go and fly, carrying the lethal message of the body through space. Thus the body was no longer only extended, it was metonymically encoded and mediumized in the spear, carrying its single-focused message across time-space the way a signal yelled carried a warning across the plain. Conversely, this allowed for the objectification of the body which, in turn, could be looked at and, as such, technologically enhanced - the hook that pierced the prey's body, could also pierce one's own body and become a part of it, an adornment, an earring, a sign of being one with things, most of all being one with things that would be threatening or limiting to the body. Embodied technologies, such as canes, eyeglasses and pacemakers were thus launched by the first 
war piercing or tattoo, and, proverbially, by the biblical fig leaf that came to conceal the body from the on-look (and thus reveal the mind's motivations to God's eye). In this way of being, technology primarily mediumizes the body by both enhancing (concealing-revealing, the fig leaf) and substituting it (the spear). This is entangled with language mediumizing the mind by symbolizing and transporting form as the field of thought - a possibility manifested not only in the functional transposition of forms onto the body but also in the proverbial transparency the biblical covering of the body gives to the mind as God reads Adam and Eve's thoughts in their technological use of fig leaves. In other words, the Platonic bi-polarization and the subsequent Cartesian decapitation of the body is encoded in the very emergence of language and technology as the two sides of the human way of being with the environment.

The mediumization of the body by human technology is an expression of the preconceptualization of thought by language. When Eve covers her body with a garment made of fig leafs, she does this in recognition of her nakedness, i.e. as a direct result of her becoming conscious of her body, in the first proverbial case of Husserlian consciousness as a consciousness of. Likewise, it is impossible to aim and throw a spear through space without imagining (i.e. holding and extending an image-thought through space-time) that the spear will carry out the intention without the direct embodied attachment of the sender. This imagining reveals the entanglement of language and consciousness as the Husserlian "consciousness of," whereby the word as symbolic distancing from what it is a signal of, and consciousness as a mental distancing from what it is a distinction of, are both interwoven and mutually supportive. The initial word/consciousness entanglement, in its enactment of distancing, opens the possibility for projection, itself necessarily temporal. Thus the gesture of throwing a spear already implies the temporal breakdown of past-present-future, no matter how incrementally or discreetly, as it enacts a possibility. Even more pertinently, it enacts the possibility of projection. The initial state of 
possibilities that the Heideggerian being-in-the-world (Dasein) implies (Heidegger, 1996 [1927]) is actualized in the temporal commitment to a single possibility - the spear is thrown (and cannot be retroactively or concurrently unthrown). Likewise, the state of being thrown in the world (central to Heidegger's understanding of being-in-the-world) becomes actualized in the consciousness of the body as a tool which affords the body's symbolic shrinking and investment in the spear. Thus throwing the spear becomes a passing-on and an enactment of the projection being-in-the world implies. And although the temporality of the spear-throwing homo sapience is far from the rocket-projecting human scientist of today, this pre-historic imagining launches the possibility for the conceptualization linguistic detachment affords. The techno-linguistic human, as gifted with a body-mind thrown-in-the-world, abides in the possibility to become conscious of his or her body-mind. This consciousness is a distancing that allows a discontinuity of the pointof-view from the body-mind and of the latter from the environment-as-the-world and opens up the possibilities for mediate relationship between those two - either to enhance the body-mind by incorporating its environment (from the garments of Adam and Eve to architecture and vehicular travel) or to metonymically reduce it and symbolically invest it into a tool (from the hammer to weaponry and post-industrial media) to pierce and transcend the world. The consciousness of the body and the fig leaf, the consciousness of the aim and the spear is the original distancing that launches the possibility for conceptualization. This original distancing emancipates the bodymind from its environment, opening up the possibility for opposition. The armed human is no longer only a tentacle of the environmental organism doing the latter's bid - the armed human has now the positional agility to turn around and face the environment as an opponent. More than that, with the unfolding of conceptualization, s/he can eventually turn around and face his or her body-mind as an opponent (in case the environment claims the latter on biological or any other functional grounds). Thus the fig-leaf-covered spear-throwing human, the human that has stepped 
into conceptualized action, no matter how primitive that conceptualization might be, is a being at war. How would s/he direct this war is a matter of care, a matter of culture.

The etymology of "war" is rooted in the Old Saxon "werran" - to confuse, perplex. The positional laxity afforded by symbolic language opens the possibility for enacted discontinuity. This means that, on one hand, words can make connections between unrelated things thus organizing deception and, on the other, they can cut through irrelevant information and forge a direct connection between things. Deception and directness intertwine in two major ways: the first is the way of logic, the way of the spear, as language forgoes the curves, obstructions and distractions, the "deceptions", of the given world to make a direct connection between two distant points. The second way is the way of poetry, the way of the fig leaf, whereby a discontinuity in the environment is used as concealing deception that reveals truth (on another mediumatic layer) - the fig leaf hides Eve's body but reveals her thoughts. However, what unites both poetry and reason as the two ways of language is their capacity for displacement (a capacity that makes linguistic description both necessary and specific to language). Thus confusion, the etymological root of "war," is a corollary of the logico-poetic displacements that language affords. (2)

Conversely, the word that stands for linguistic conflict, "polemic," is etymologically rooted in Polemos, an ancient Greek Daemon of war who, from an ancient symbolic personification of physical war has crystallized into a concept of linguistic confrontation across contemporary languages. A polemic today is an intellectual debate that has no physical connotations, making the linguistic entanglement of the concept of war even more explicit than the etymology of the latter. Indeed, war becomes possible only with linguistic conceptualization a non-verbal animal fights, chases, struggles and escapes its environment, but does not organize this struggle into sustained conflict. By adding consciousness to perception, thus agglomerating perception-conception (Merleau Ponty), language not only supports perception in its focusing on 
forms and pulling them out of the environmental mesh, but also sustains that distinction as the thingness of things (Heidegger). In emancipating individual forms and sustaining them beyond the immediate perceptual enmeshment (with the environment), the conceptual enactment of language pauses the flow of being and then, upon committing the concept to thinking, accelerates that flow converting it into time. Time is thus a category of thought - war as a sustained action is only possible in time, therefore it is contingent on the being of language. Heraclitus called Polemos "the king and father of all," making "slaves on the one hand, the free on the other." (Heraclitus; Kirk 2010, 52) Read in its fragmentary solitude, this quote seems to be alluding to the opposition of things - Polemos as the progenitor of things opposable, the slave and the free. Things can only be summed as "all" in the sustained distinctness that the distancing of language affords - a distinctness that opens the possibility for opposition, or Heideggerian confrontation:

"...for Heidegger this confronting constitutes the fundamental condition to our existence, but not in the Darwinian sense of a struggle for existence as the survival of the fittest... For Heidegger, confrontation describes our Being as interpretative beings, as beings for whom the meaning of other beings - and of Being itself - is at issue... Our Being is hermeneutic, and polemos as confrontation pertains to this interpretative manner of Being." (Fried 2000, 1516)

This hermeneutic manner of Being is grounded in perception - being a perceiver means being a descriptive interpreter (the perceived - a descriptive interpretation formed and formatted by the perceptive body's capacities). Therefore the Heideggerian polemos was not necessarily language-bound - the confrontation that can linguistically frost into opposing concepts, is already a dynamic principle of perception, the confrontation with the world we are originally thrown in as beings. But as was already discussed, language precedes language, the techno-linguistic layering of the animal world begins before technology and language; mediacy is wrapped in the animals' im-mediate action like a seed that would sprout in human making that reverses the layering into immediacy wrapped in mediate action (the deliberate crafting and mastering of the spear then 
used for immediate action). Mediacy therefore foregrounds the "interpretative manner of being" as activated by polemos. However, the original (sensual) confrontation of being with the world can only be a battle; war unfolds with the unfolding of conceptual language. And, as it does that, the word "polemos," that for Heraclitus symbolized the original energies of confrontation that set things apart, recedes to the cognitive confines of language, giving way to the word standing for the cognitive state of confusion "war" to advance into the field of action. This curious reversal of action and language may seem random and circumstantial but it nonetheless outlines trajectories of thought concerning war and language that are invariably entangled and culture-bound.

"Culture" comes from the Latin "cultus," meaning "care." Heidegger's Being concerned with Being and the meaning of other beings, already extends itself with care. (Heidegger 1997 [1927]) Our being in the world, by definition a concerned being, is already a being unfolding toward culture. And since this being is linguistic, it is also a being at war. Concern as a way of extending, and eventually projecting ourselves, thus has already an intent fulfilled in cinematic media. While shadows and reflections are bound to the projected body and thus confine our care and conflict to our immediate surroundings. Only with industrial technologies projection frees itself of the original projected body and becomes a global concern (both caring and menacing). The projection technology becomes the intermediary, the bona fide medium, between the original body and its projection, e.g. a physical apple and its photographic projection on a movie screen. Thus, strictly speaking, the cinematic projection is no longer a projection of the original body - it is a projection of the projection (the photographic capture) of the original body. Thus industrial projection, cinema, becomes the embodiment of the concept of projection itself.

The throwing of a spear - the enacted thought projection is also the first gesture of care (crafting of the spear) toward war (systematized confrontation with the environment). 
The care that mobilizes the crafting of the technology allows for confrontation as facingup-to, i.e. as an emancipation. The spear-throwing being is no longer a chance victim or predator but a warrior, an equal player in the theatre of life. Actualized projection, the concept made physical emerges as a gesture of war. Thus industrial technologies as automated means emancipated from the human body mark the apex of cultural unfolding of actualized projection. Cinema, an industrial technology that turned concept into reality, emancipating projection itself, therefore emerged as war-bound. Unbound technologies that allow for unbound violation are warbound.

Before the first hominid threw a spear, s/he held a stone as a carving tool. Before the first drawings of a bull graced the walls of a cave, Neanderthal females imprinted the walls of their caves with their painted palms. And although we can still see those direct body imprints of the first known murals today, across time and space, from them to an ancient Egyptian sculpture of a pharaoh, ancient art was not memorial and representational in the way these terms are understood by a conceptually fluent being. These were body doubles re-presencing the original rather than empty outlines referring to an absent or located-elsewhere body. Likewise, ancient language was the attractor and mediumatic channel of the energies that animated the original they mimicked an incantation, a word of blessing or a curse conducted the power of the physical event they summoned, they are not mere threats or metaphors. Yet the spatio-temporal multiplication they eventuated ushered the possibility for detachment and displacement of form/thought/language actualized in conceptualization. Ancient mimesis is invocation, not reference - it contains the summoned energies here and does not point at them there.

As early as the ancient Egyptian creation myths the processes of physical and linguistic propagation are distinguished in two separate yet complementary creation myths. While god Atum created the world through his all-encompassing physicality in a hermaphroditic act of 
masturbation (Wilkinson 2003, 18, 99), Ptah created the world by thinking in his heart (the Ancient Egyptian seat of thought) and speaking with his tongue the names of his creations.

The combined use of the body as medium and of spoken thought as language to create their environment makes ancient Egyptian demiurges the original modellers of what Don Ihde would identify as human technologies. Unlike other animals who only modify their environment, humans use technology to relate to their environment and magnify it. (Ihde 1993, 51) Thus augmented reality would perhaps be not only the appropriate name for a specific mediumatic technology but indeed, a qualifying feature of human technology as such. Humans severed the hand of Atum and stole the speech of Ptah to craft their tools of technological extension and linguistic abstraction. These independent (by means of amputation and displacement) technologies of body and mind became ways to enact the creative principle in the already given environment. As given and contiguous to the body, the environment cannot be thought of as conceived by the human who is only a part of it - logic, as the bona fide conceptual way, cannot reconcile the discrepancy of something emerging from its part, containing only partial information of the whole (although eventually scientific thought would arrive at the holographic principle through technology and, thus through technology, become capable of conceptually sustaining a poetic possibility). Human technology affords a self-induced amputation from the environment, a screening out/shielding of the human body-mind that thus enforces an otherness to the environment that allows for the human to relate to it and therefore move independently through it, thus acquiring the capacity for dramatic rearrangement and augmentation. That relational independence becomes possible because of the conceptual distancing made possible by language. But just as the human has positioned itself as indispensable to resolving this environmental crisis it has provoked, the machine would position itself as indispensable to 
resolving the environmental disconnect it has enacted. (By environmental crisis, I mean a critical displacement or misalignment of energies, not necessarily referring to specific current events).

This brings us, oddly, back to elementary particles. How? Conceptualization makes possible the development of industrial technologies, distancing not only from the field of things (as in traditional technologies) but also from the producing body itself - the stick of the ape can be controlled by a mechanism standing for the body of the ape, who can be miles away; connecting not only to the resource from which one is being initially distanced, e.g. the ant hill, but also, as is linguistically congruent, to other sticks poking other ant hills. This distancing from the body and connecting to other technologies, makes possible the safe exploration of fields that are mediumatically inaccessible and dangerous to the body.

Concurrently, language makes possible the conceptual speculation over possibilities of layering. By hovering over things, symbolic language blankets the field of things - it is, as discussed, not directly related to anything and therefore its presence is blanketing rather than extending (as is technology's). By blanketing, symbolic language both covers and signals things and directions, i.e. it layers reality so to speak. The mediumship of technology-rendered-linguistic emerges in a layered reality and vice versa. Those are the two sides of linguistically mediated orientation in the world. It is only through language that the human can uncover the layering of reality made possible and explicit through language. A conceptualization and respective technological manipulation of elementary particles is thus impossible outside of language. This is not to say that language is the only modality to access the force field of what we conceive as elementary particles - it is to say that it is the only modality through which we have collectively accessed that field within the current Western cultural parameters. In order to conceptually elucidate and henceforth technologically access elementary particles, we had to rely on advanced abstraction, i.e. we had to go as far away from embodiment as possible in order to conceive of 
ways to access its elements. The notion of elementary particles became available to us as soon as we could reason through language - ancient Indian thinkers, Buddhist and pre-Socratic philosophers all had their version of atomism explaining the consolidation of the material world. Democritus, just as the Indian atomists before him, conceived of an atom, as the smallest indivisible particle aggregating in phenomenal matter, in response to the mathematical paradox the notion of infinite divisibility placed on the behaviour of natural bodies, as famously elucidated in Zeno's paradox. The notion of a basic universal component of matter makes the fantastical idea of the alchemical transmutation of base metals to gold known as crysopoeia (from Greek khrusōn - gold, and poiein, to make) not only conceivable but achievable (in principle) when Otto Hahn achieved chemical transmutation of Radium into Barium and thus launched nuclear fission. The splitting of the atom not only opened a new conceptual space for reintroducing the concept of infinity into the study of matter, but it also inaugurated a new way of extracting energy from to the sub-sensory field of matter. The technology that makes particle physics a practical reality and not just a theoretical possibility is the extension of the same thought that conceived of the atom from the facts of phenomenal matter.

The necessarily linguistic mediumship of technology in/of layered reality makes explicit a certain temporality specific to the distancing-connecting blanket of language - it is a distancingconnecting temporality that delineates the axis past-present-future and coordinates it to lengthwidth-height. The spacial distancing affords the emergence of Imaging-Geography and the temporal distancing affords the emergence of Memory-History. It is within those parameters of measurability that conceptual travelling is technically possible both to the cosmic and to the quantum. However, those conceptual modalities of space-time are not the only ones available through language. The body-lingual point-of-view, for instance, abides in a realm that, by extrapolation, shouldn't have much to do with the measurable parameters of Imaging-Memory. An 
intimation of the validity of this supposition is the experience of fluctuating "subjective" time, and even more explicitly, the unruly "spacio-temporality" of dreams where events can unravel every which way, including simultaneously (within the same moment) and coincidentally (within the same space). Dream as the entrance of poetry to language defies the syntax of conceptualization - it offers the other side of conceptualization, the floating form unfettered by the physical framework of validity. At the other end of the conceptual journey into physical reality, we encounter the elementary particles that, just like the phantoms in a dream, behave every which way, here-there now-then, defying the linguistic expectations of the instruments conceived to observe their behaviour. Or is this so inconceivable - dreaming makes explicit a way of being beyond the sequentially measurable and thus prepares the cognitive grounds for the emergence of such ideas as the Quantum mechanics' challenge to local realism (the classical scientific view that any object must have pre-existing (measurable) value) by stating that it is impossible to predict a measurement of an elementary particle before the measurement is made. That would imply that, before the commitment made in the encounter of the elementary particle with a measurement instrument, the former remains in a suspended state of all possible states or destinations as exemplified by the paradox of Schrodinger's cat (both dead and alive before the observer opens the box that contains it - a state of possibilities reminiscent of a Heideggerian Being-in-the-world). The multiverse proposition is one solution whereby the cat does not collapse to either state but instead the two versions of the event, the observed live and the observed dead cat, continue to exist in parallel tracks of reality. Conversely, the realistic solution of the event would have the cat collapse to one of the two states upon encountering the measuring instrument (in this case, the observer) while the Neumann/Wigner interpretation would attribute the collapse to the consciousness of the observer. In any of the proposed solutions each reality commitment entails both rational and oneiric thinking enfolded into each other. 
By exploring empirical reality on rational terms, the scientist arrives to empirical reality on oneiric terms reuniting the mind-world with the conceptual universe in a new Moebius twist. The measurability of the sensible world leads to the idea of measurability beyond the sensible and by hitting the boundary of measurability in the atom, the scientists enter a domain of measurability that nonetheless rests on immeasurability - a new level of existence that resonates with the principles of dream more than with the laws of wakefulness. This made the suppositions that explain quantum theory through the interaction of elementary particles with consciousness (the many-minds solution/ Neumann/Wigner interpretation) valid in a whole new, non-scientific, poetic sense. On the other hand, particle physics arrived at a new "atomic" revelation - the photon - whose speed (the proverbial speed of light) "makes" it massless and formless. This sets a new frontier for the sensible - the frontier of form itself carried by nothing less than light itself, by what makes objects visible. It wouldn't be farfetched to theorize that the photon is the basis of matter, the departure of matter as it moves toward both mass/tangibility and visibility. Such proposition would not only harmonize with both the canonical E=MC2 (conditioned on the speed of light) and quantum mechanics as the field concerned with the photon, but it would also poetically rhyme with the Torah's creation story: "In the beginning God created the heaven and the earth. And the earth was without form, and void; and darkness was upon the face of the deep... And God said, Let there be light: and there was light." (Genesis 1:1)

The visible would thus be already entailed or enfolded in the invisible and vice versa. The formless photon would be the state of departure for the emergence of form that its light would then come to illuminate. Conversely, the formless photon, whether morphologically related to the other elementary particles or not, remains the boundary of the world of form both by virtue of its massless formlessness and by virtue of its power of illumination, of rendering things visible. All this emergence of the phenomenal world from the element of light was triggered by God's 
spoken word resonating, across cultures, in Ptah's creative utterance. The creative language of God is not mere naming, it is a specific vibratory force that begets form. Ancient language (language before language) is thus not merely an arbitrary set of symbols but a system of vibrational keys that bring forth and sustain the world. This ancient view of language that ties it to light as the underpinning of the phenomenal world (and eventually, to elementary particles as the underpinning of the physical world) has not only inspired ancient Gnostic practices, such as the Kabbalah, but also brings a new clarity to the realities implied by Heidegger's view of the word as sustaining thingness and Foucault's view of discourse as a socio-historical force.

Empirical reality, the body of Atum generated by the visceral hand-to-penis touch, has unfolded matter poetically both in the miraculous flourishing of the flower and the mysterious vibration of the photon. Poetic reality, the birth of the sensible world from the heart-felt dream of Ptah, has aggregated into the magnificent bodies of the world, all through the manifold moebius twists of language-form and matter-form unto each other. Poetry as discontinuous begetting is thus the principle of divine technology - both Atum and Ptah create the entire manifold of the world out of a single gesture. Even the mechanical thrust of Atum is miraculous in the intricate abundance it initiates. Poetry as music and metre, as that which carries forth the technology of the muses (music as flow) and the mechanics of the physical world (rhythm as counting) makes explicit poetry and reason as the two ways of language. Together, the body of Atum and the word of Ptah bestow the gift of the mind-body as a conduit of technology manifested in the tool of craft (the hand) and language (the mouth). Language-technology becomes the mediumatic way of being.

This fantastical view is not a scientific acquisition - it becomes possible only because science is embedded in a much larger field of thought. When Democritus proclaimed his atomist vision of the world, he used logic to make a poetic leap from the sensibly known to the sensibly 
unknown. Millennia later the sensibly unknown became technologically accessible and therefore mediumatically sensible confirming the ancient leap. Scientific technology gave science a positive access to the sensibly unknown but precisely intuited by the linguistic mind. However, technology as the medium of the body, and language as the medium of thought, i.e. the technolinguistic condition as mediumatic of the body-mind, is made most clearly manifest in the arts which bring the distancing-connecting projectiles of language back into the linguistic manifold of poetic actualization. Thus science confirmed on its own terms what the artful ways of myth have made clear all along. And yet, science has distanced itself from myth so far in the process that its confirmation no longer makes organic sense, thus threatening to highjack the entire field of thought and forever disengage it from sensible reality.

\section{Methodology}

How does all this relate to methodology? As far as the present work is necessarily conducted within language, it has to approach its questioning by taking into account the conditions of language, the interpretational background, where this questioning unravels. The first condition of language is layering. Poetry and reason are enfolded in each other and our conceptual oppositions are only a manner of speaking. Oppositional concepts are dynamically enfolded into each other. They are not in steady relationships that could yield more solutions if examined as relationships rather than concepts, for example. When considering the oppositional methods that reject one part of thinking or another (e.g. logic vs. intuition), it becomes apparent that those rejections can only be approximations, degrees of linguistic positioning. The careful examinations of such, experientially false but linguistically correct, dichotomies reveal the actual polemos - of being and nothingness of language and silence, of the known and the unknowable. 
This becomes historically possible only now, as those fields have unfolded far enough to reveal how their divergent trajectories are beginning to re-convene.

Squarely speaking, cinema is a technology of the moving image. Cinematic technology, as both production and communication technology, is mediumatic. The medium as a conduit of information pertains both to the physical and the linguistic way of distancing/connecting. The moving image, in turn, is a phenomenon of the mind. The mind as the screen on which we organize our experience can be both sensual and symbolic. The physical/linguistic and sensual/symbolic belong to both the rational and the poetic. To address the question what does cinema make possible, all those aspects of the cinematic must be accounted for. The research of the mediumatic technology of cinema as a language that articulates a phenomenon of the bodymind is therefore concerned with a reality-generating technology. The question of reality is inevitably a question of both epistemological and cognitive study, if the manifold of such a complex construct is to be approached justly. Therefore the question of cinema as a technology of the moving image requires an approach that accounts for, or at least does not close off all the ways in which poetry and reason have interwoven the techno-linguistic being. Thus, instead of regarding the world as a composite of separate opposites positioned in mutual (necessarily spatial) exclusion (objective and subjective, inside and out, and more pertinently to method, description and prescription), it can be viewed as a tensional dynamic in a field of forces. The wavering flow of poetry and dance is complemented by the discernment and precision categorical tools afford, with the condition that they remain recognized as navigational tools and not vehicles for final answers. Poetry and reason as dramatized in art and science, and kaleidoscopically multiplied by the theoretical tools in-between, call for an approach that commits to balance, not in the mechanical sense of rest, nor in the dancer's way of flow, but rather a balance of both rest and flow - a static flow, a dynamic rest - that can weave a semantic fabric without sealing it into a 
monolithic system (as Modernity would dictate) or crumbling it into a fragmented expanse (the Post-modern way).

The radicalization of the techno-sensible and the poeto-conceptual has been theorized in terms of shared/objective and experiential/subjective realities. This, often mutually exclusive, radicalization would become most explicit, and therefore reconcilable, when discussed in contrasting phenomenological and scientific terms - two (linguistic) domains that often detract each other for the same reasons that make them compatible in the present questioning. Phenomenology questions the world from the first person within whose field of perception the world unfolds and who, bound to this unique position, accounts for the unfolding in terms generated within that same field. Phenomenology regards the world as native to the perceptual field. By contrast, science regards the world in terms of a universe, as an expanse of measurable phenomena whereby the subject is only an objective instance that cannot be, strictly speaking, accounted for as a subject. Science regards the world as exterior to the subject, and the subject as an objective part of the world. Although those two positions have been viewed as competitive more often than not, when travelling to the end of a phenomenological argument, what is left is an intimation of something larger than the (knowable) subjective field, and vice versa - when travelling to the end of a scientific argument, what is left is the view of the (scientifically, i.e. measurably) unknowable subject. This bordering with the unknown and the mutual tensions it exerts, is the ground that makes those two positions complementary. The methodological advancements of science has brought us to this edge where the view to the validity of the other side has not only begun to clear up but also to become indispensable for the consolidation of new findings, where "...only a balanced and disciplined account of both the external and experiential side of an issue can make us move one step closer to bridging the biological mind-experiential mind gap." (Varela 1996, 343) 
Thus this is not a farfetched proposition. On the reverse side of research, Francisco Varela's Neurophenomenology - a method of engaging techno-scientific and phenomenological tools to tackle questions of the brain-mind - has already initiated a dialectical reconciliation between the two ends of rational questioning which have become indispensable to each other with the advancement of Neuroscience and its increasing contingency on subjective reports. The limitations of positive scientific research are both relaxed and disciplined by phenomenological enquiry and vice versa as "phenomenological accounts of the structure of experience and their counterparts in cognitive science relate to each other through reciprocal constraints." (Varela $1996,343)$

What is at stake here is no longer the integrity of language (as a way of poetry disciplined by reason) but the integrity of reason as a way of language disciplined by scholarly research. In other words, while the discussion of arts and sciences as the disciplinary expressions of the two integral ways of language (poetry and reason) pertains to the integrity of language itself, the discussion of phenomenology and techno-science as a radical expressions of the two ends of scientific questioning pertains to the integrity of reason as a way of language. Science as a sublanguage necessarily puts forth a certain syntax that defines the paradigmatic limitations of executability within it (e.g, there are certain mathematically possible statements untranslatable in dance and vice versa). This makes the sub-linguistically unknowable easily demonstrable and also delineates the cognitive limitations of the sub-language. Cognitive science and phenomenology are the two remotest dialects of the rational study of the mind and as such mark the edges of its body. Likewise, although contradictory, the laws of Newtonian time and space, Einsteinian time/space and quantum physics' entangled temporality are not mutually exclusive they pertain to different mediumatic layers of physical reality. Thus, making absolute statements within a certain sub-language becomes nonsensical, but the dialectical reconciliation of its 
dialects is indispensable, if not for securing new findings, at the very least to avoid the temptation of such totalizing statements.

But, as we already discussed in Chapter 1 , there is a qualitative difference between the rational and the poetic way of being with language. The rational way - spacio-temporal and conceptual - can only travel by abstraction and arrive to a destination by following a sequential procedure. It is a way of travelling by distancing, mapping-describing and recording. Rational language is subverted by disorienting - the wrong move or direction can lead to a false arrival, false by virtue of the correspondences that describe it. The integrity and alignments of rational language are therefore contingent on making correct correspondences - on correctness. For Heidegger the understanding of truth as correctness begins with Plato "for it is he who first conceives of thinking Being as a going "beyond" the beings of experience to their being-ness, which he conceives as their what-ness, their see-ableness, their Idea." (Richardson 2003 [1963], 308) This becomes possible by means of a conceptual dislocation: “...a consequence of $\varphi v ́ \sigma ı \varsigma$

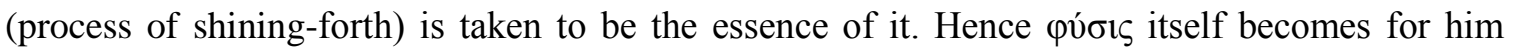
that-which-is-to-be-seen, a being (cílos). Being thus becomes conceived (emphasis mine) as a being. Likewise truth, no longer non-concealment, becomes correctness of view, conformity with the Ideas." (Richardson 2003 [1963], 308) Thus, ironically, the very inauguration of conceptual language as the correct way to knowledge is enabled by a dislocation whereby it not only reveals its falsifying nature but also projects it onward. Conceptual thought as the falsifiable aspect of language is revealed in its ontological reliance on correctness. Thus conceptual language and respectively science as its most accomplished sub-language cannot afford mistake - mistake can be lethal when the correctness of the path in a proverbial forest means the difference between avoiding or falling into a precipice. Correct correspondence as a way of mapping presumes that the world consists of discrete irreducible instances that can be accounted for by exactly 
corresponding irreducible linguistic instances and their correct relationships. This logical implication of conceptual language becomes most explicit in Russel's logical atomism which claims that truth can be arrived at by logical analysis based on this premise of such an overlap between language and reality. Although Wittgenstein, who inspired Russel in explicating logical atomism, later withdrew from it, the value of this theory rests not so much in its applicability or falsifiability, as the case may be, but in the way it radicalizes and makes explicit the implicit premises of conceptual language itself. Pure conceptualization, by framing a perception of reality whereby language overlaps perfectly with the world, coils on itself as the truth which thus becomes contingent on its correctness. In other words, by foregrounding itself as reality, conceptual language invokes the danger of closing itself off and turning the mirror of reality away from itself. Conceptual language, in its extreme, becomes the reflected, framing the world in its image. In a world thus flattened, where depth of focus as the way of seeing becomes obsolete, the original ancient Greek meaning of knowledge as insight (gnosis) becomes nonsensical. Understanding becomes a mere fancy in a place where there is nothing to stand under, or rather where every flat expanse is a precise replica of the one underneath and can be deduced by association. The only way to navigate such a logico-linguistic universe is through knowledge as re-cognition (from the Latin cognoscere). What this logical reduction of language reveals is that re-cognition, as the assignment of significance of a known form, pertains to reason. Thus reason affords the alignment of the mediumatic layers of symbolic reality (e.g. the assignment of a symbol to a form), and therefore, for its correctness to be authentic. It must eventuate this alignment, as in the already discussed stabilization of thought - designation as a way to focus and channel energies, not as a container of the designated thing. But historically "the sculptor of language was not so modest as to believe that he was giving things designations he conceived rather that with words he was expressing supreme knowledge of things." (Nietzsche 2004 [1878], 
16) Knowledge contained in language - conceptual language reflecting upon itself, can only hold sway when searching for answers about itself. Projecting the findings of that searching onto any other form of reality (e.g. perceptual, the history of which had produced the idea of thing-initself) becomes inevitably an act of falsification. Thus the very independence of language from any other reality, coupled with its capacity to describe and relate to other realities, imbues it with the capacity for falsehood. Indeed, deception is an exclusive property of language. The linguistic power of deception had fuelled Nietzsche's falsification thesis of language as rooted in generalization (conceptualization) as the operating principle of logic and reason:

"Logic too depends on presuppositions with which nothing in the real world corresponds, for example on the presupposition that there are identical things, that the same thing is identical at different points in time..." (Nietzsche 2004 [1978], 16)

So can language, the source of falsehood itself, have anything to do with truth? As the usher of opposing values, it must somewhere also engage with the opposite of falsehood. But since it is that which is signed by falsehood, then language must be most true when it assumes its falsifying signature. Language assumes itself as deception in poetry; art as the discipline of poetry is where this truth of language is pursued. As Picasso would observe, "art is the lie that tells the truth.”

Art is concerned with beauty as the truth of language, as the truth of form. Poetry as the way of beauty reconciles the (logically) irreconcilable. It reveals language's capacity to transcend (its own) reason, and bring us to the edge of the known - to silence. Poetry does not designate, it lifts away the designations. It doesn't orient us to things, but once oriented, it reveals them to us in their unfathomable wilderness, as wondrous singularities that flare up in the vastness of silence. While conceptual language, by designating, enables recognition and the uncovering of mediumatic layers, one by one, step by step, poetry makes all those layers instantly dissolve in 
the wondrous phantasmagoria of dreams to intimate that which is beyond language altogether. Concept is a way to recognition and uncovering, poetry is a way to revelation and insight.

Derrida claims that "poetry is to prophesy what the idol is to truth" (Derrida 1978 [1968], 67), but this statement gets entangled in its own eloquence (two nouns that start with "p" and involve non-linear time do not necessarily belong to the same field). Poetry is the language of prophecy - the only truthful-in-its-illusionary way of articulating an intimation from beyond temporal linearity, from the non-linear perception of dream. At the other end, poetry is also the language of madness - that which is not only outside of logic but also outside of any consensual field. Madness is the final frontier of poetry - the place where the original cacophony of dreams shines through in its devastatingly fleeting singularity, the very essence of time that haunts us in our frantic search for meaning. Only by passing these phantoms through the stabilizing filter of thought-language we can pause and look at the oncoming time, without disintegrating in its nonsensical onslaughts. But the madman, shouting nonsensical sentences, reveals the hopelessness of this manoeuvre. In his work on madness, Foucault called for letting something outside of reason speak (Foucault 1988 [1961]). Art is the only sanctioned discipline where prophecy and madness are reconciled in a language that can articulate both nonsense and insight without traumatizing the capacity of language to communicate. This is why this work's methodological commitment is grounded in artistic method and poetic language as much as in rational approach.

Long before the Western scholastic tradition embraced scientific rationality (a gradual process arguably inaugurated by Plato but rapidly crystallizing since the Industrial Revolution), there has been the Sanskrit "rtih" (mode) that became the Greek "arti" (just), the Latin "artus" (joint), and the Armenian "arnam" (make). Thus art arrived to us as the just and flexible mode of making. As such, it has been traditionally designated as the primary approach to knowledge not 
because of its commitment to truth, but because of its commitment to beauty as the truth of language, as that which, according to Marcuse, reconciles opposites without violence. (Marcuse 1971 [1969], 33-34) Unlike science which advances by reduction, art expands by inclusion. Scientific quality is a question of description, artistic quality is a question of intensity; scientific quantity is a matter of measurement, artistic quantity is a matter of balance. In order to use the precision that science affords (but avoid the fixating dangers of precision) and to use the flexibility art affords (but avoid the danger of disintegration), the current work is as much a questioning as it is a methodological intervention that searches to art-fully balance rational descriptions and avail itself of measured intensity.

As the consensual field that disciplines poetic language, art has not been primarily invested in the mimesis of reality, as Plato famously surmised (Plato 2004 [380 BC]). If at all concerned with mimesis, from the very first realistic depictions of animals on cave walls to abstract painting, art has invariably been invested in the mimesis of the human mind (in the broader sense of mind as the embodied screen of apprehended form, including perception and language assembling the mind-world), and especially concerned with rendering visible that part of vision which is not optically available. Aesthetics of thinking is therefore as much a part of the artistic concern as is the aesthetics of the senses (the common unspoken designation of aesthetics). Artistic thought, as an expression of such aesthetics, is inclusive not only of all forms of thinking but also of the falsehood of those forms, the mistakes and aberrations they afford. Thus, while techno-scientific thought's utilitarian concern with how things work provides little room for mistakes and false moves, artistic thought is contingent on the aesthetic possibilities mistakes afford. The Japanese wabi-sabi which deliberately enhances cracks, defects and breaks in the work of art is not a mere stylistic curiosity - it makes explicit one of the core principles of art as a way of generating meaning from the multiplicity of human experience. Wabi-sabi draws 
attention to the open-endedness of an artwork which is a metabolic entity that can be enhanced by aberrations, insults and onslaughts. It underscores the physical weight of the poetic crack and the actual leap that cuts across the fabric of the space-time continuum. This approach has defined Walter Benjamin's dance with knowledge, as articulated by Richter:

"...his thinking focuses on the ways in which the process of cognition depends in its very formation on what has not been fully understood... Put another way, cognition can be what it is only when it actively encounters and openly engages its abiding blind spots... These blind spots - Benjamin's leaps or cracks - constitute the defective but necessary architecture of all his concepts." (Richter 2007, 63)

The methodological aspirations in questioning the manifold of cinema are to balance poetry and reason through reconciling a multitude of angles in a way that affords open-endings, mistakes and incompleteness. By taking the risk of multiple perspectives, a research would inevitably generate mistakes - the only way to mobilize these mistakes as openings and not as dead ends, is to make them part of the fabric of the work itself - the leaps and cracks that allow for further exploration. The incorporation of poetic language and artistic techniques can be a guarantee that the certainty of rational conclusions is continually destabilized without being undermined. This is neither a novel idea, nor an unprecedented approach. In his ever shifting, uniquely dramatic writing style Nietzsche has long ago inaugurated a philosophical technique by means of which he "...manoeuvres and plays with both the "universal and eternal truths" and his own personal convictions, multiplying to infinity each one of his ideas, could be read through perspectivism that, according to Nietzsche, represented "a basic condition of life itself" and the respective principle of interpretation." (Zhabilova 2010) Zhabilova identifies Nietzsche's polyvalent methodological intervention as "an optics assigned by life itself" that is not only "a daring aesthetic experiment, but also an experiment with an enormous epistemological potential, 
because the optics of multiple points of view gives a fullness of knowledge." (Zhabilova 2010, trans. mine)

Similarly, the process of the current questioning is often grounded in the written work (vs. the written work describing a process). The dynamic interplay between language as making and language as description continues the Nietzschean rhetorical tradition as far as the tracking of experience as a way of knowing leads to the convergence of the researcher and the researched. However, it also honours thinking as experience, an idea derived from (or at the very least inspired by) the Kantian critique of aesthetic reason (Kant, 1914 [1790]). This places Nietzschean thought in a dialectical synergy with Kantian epistemology as only one of many synergies that the current approach allows for with the full awareness that they may be abrasive to traditional interpretations and established positions. However, even in its bid to synthesize his thought with that of his arch-enemy, this approach still abides by the wide Nietzschean platform that implicitly accommodates any position even that which antagonizes it, as long as it is not totalized. Nietzsche described the genuine philosopher as someone who "must have been critic and sceptic and dogmatist and historian and also poet and collector and traveller and solver of riddles and moralist and seer and 'free spirit' and almost everything in order to pass through the whole range of human values and value feelings and to be able to see with many different eyes and consciences, from a height and into every distance, from the depths into every height, from a nook into every expanse." (Nietzsche 2003 [1886], 326) Nietzsche's reverence for the variety of human ways of knowing (he opposes to the rational dogmatism of science) echoes in Feyerabend's methodological anarchism challenging the scientific approach from within. He calls for an epistemological eclecticism whereby there is "no idea, however ancient and absurd, that is not capable of improving our knowledge" that also serves the prescriptive imperative of science is based on the principles "anything goes." (Feyerabend 2002 [1975], 33) Feyerabend argues against 
the purist methodological aspirations of science in claiming supreme epistemological positioning - a positioning that is not only inhumane, but also detrimental to the development of science itself. A commitment to knowledge cannot, either truthfully or correctly, dismiss the variety of human thought to the exclusion of one single way of study. Moreover, Feyerabend argues that the scientific values of efficiency and progress would not be undermined, but strengthened, by an eclectic methodology. While a straightforward, uncontaminated mathematical formula might be the best way to calculate the velocity of a simple body in motion, the more variables a question involves, the more it calls for cognitive flexibility. As we exit the plane of Euclidean geometry and enter curved space, we arrive at strange places, like Einstein's dreams, where the irrational and the rational collide to yield a scientific answer.

Cinema - a technology, a language, a medium and, above all, a simulacrum of life as image in motion - is a complex phenomenon, full of curvatures that defy single perspectives. This is why the approach of the current exploration echoes Nietzsche's and Feyerabend's understandings of epistemological commitment to any way that may illuminate a curve in the manifold of cinema. However, both perspectivism and anarchism remain silent about synergy how does a multitude of angles and considerations come together in a dynamic whole? What kind of forces can safeguard the integrity of a question open to anarchic influx? Hegelian dialectics offers a technique that, applied itself as a dialectical opponent to anarcho-perspectivism and extended to more than two aspects of a manifold phenomenon, promises to build a peculiar tensegrity structure.

Tensegrity is a term coined by architect Buckminster Fuller to designate a principle of structural dynamics based on the interplay between tension and integrity that is a “comprehensively operative discontinuous-compression, continuous-tension structural systems as 
inherent to synergetic-energetic geometry and its omni-rationality of vectorial, ergo energy, accounting.” (Fuller 2001, 240)

In his introduction of tensegrity, Fuller traces its presence in structural organization from the quantum to the cosmic in a geometrodynamic view of the universe that reconciles old dichotomies, including poetic intuitions and mathematical certainties:

"This break-through accrues to an age-long, comprehensive emergence, and convergence, of a myriad of world-around technico-economic, evolutionary realizations originally initiated only in the extraordinary moments of purely poetical lucidities of man as ephemerally and only tenuously accredited "couldbe's..."” (Fuller 2001, 240)

Tensegrity, the synergetic alliance of tension and integrity, proved even more comprehensive than Fuller envisioned - it transcended geometry through the work of Donald E. Ingber who identified it as an organizational principle of organic structures. (Ingber 2003) It has since been applied to muscular-sceletal and cellular mechanics. Tensegrity describes the tensional integrity between solid and flexible components, such as the bones and muscle/tendons, that build up a dynamic structure.

Transliterated methodologically, tensegrity seeks to establish tensions that promote structural balance between opposing notions, introducing an alternative to the Hegelian "battle and union of opposites". Tensegrity allows for the collaborative clash of such opposing ways as positivism and phenomenology, empiricism and rationalism, reason and poetry. Thus, to the phenomenological intentionality, tensegrity responds with a hybrid approach involving tension as a multi-directional force, in-tension. Derrida's post-Structuralist elucidation seems to outline a similar programme:

"Our intention here is not, through the simple motions of balancing, equilibration or overturning, to oppose duration to space, quality to quantity, force to form, the depth of meaning or value to the surface of figures. Quite to the contrary. To counter this simple alternative, to counter the simple choice of one 
of the terms or one of the series against the other, we maintain that it is necessary to seek new concepts and new models, an economy escaping this system of metaphysical oppositions. If we appear to oppose one series to the other; it is because from within the classical system we wish to make apparent the noncritical privilege naively granted to the other series by a certain structuralism.” (Derrida 1967, 19)

The concern with metaphysics has reverberated from Nietzsche to Heidegger, throughout science and post-modernity. Scientific method does away with metaphysics by removing the question of "what is" from its inventory. The exclusivity of "how" is the elegant methodological solution of scientific research. However, Nietzsche rejected metaphysics precisely because of its alliance with science's totalizing ways. He imbued his language with poetic undertones that made explicit the conceptual underpinnings of metaphysics. Heidegger advanced the project of linguistic destabilization that lay bare the axis metaphysics - concept revealing that it is conceptual language rather than the philosophical concern with the "whatness" ("essence") of things that defined the techno-scientific mindset. Thus, strictly speaking, epistemological monism and cognitive dualism do not belong to metaphysics, but to language itself - to the conceptual part of language. The current approach not only regards oppositions as belonging to conceptual language, but also considers conceptual language an integral part of language itself and, as such, an integral part of this study.

Derrida continues:

"Our discourse irreducibly belongs to the system of metaphysical oppositions. The break with this structure of belonging can be announced only through a certain organization, a certain strategic arrangement which, within the field of metaphysical opposition, uses the strengths of the field to turn its own stratagems against it, producing a force of dislocation that spreads itself throughout the entire system, fissuring it in every direction and thoroughly delimiting it." (Derrida 1967, 20)

Although a structural principle, tensegrity does not aspire to static symmetric stability as metaphysics and classical science do, but it also does not aim at dismantling that stability. 
Constructing and deconstructing systems of knowledge are paths that have been consistently pursued throughout the history of thought, and tensegrity builds on that history. Derrida views everything as a textual continuum. Destructive and de-structuring to the text as a metaphysical construct, he creates a parallel universe that lays out text flatly ad infinitum. Tensegrity pulls and aligns threads of meanings in a delicate balance, on various planes of formal intensity - it does not reinstate hierarchy in the classical sense but folds the universe back into a multi-dimensional volume that can now account for the mediumatic layering of formal organization that poststructuralism sought to do away with. This is a precarious balance that, when static, can tumble down at the lightest blow - its very delicacy and non-symmetry prompts it to motion, to switch perspectives, to whirl around its axis and assemble a kaleidoscopic world, necessarily imperfect and unfinished, full of cracks, defects and shortcomings that offer glimpses to the infinity beyond any structure. Saturating a research with a multiplicity of angles and approaches, a tensegrity approach would produce an overflow of meaning that would aspire to break the sound barrier and reveal the silence on which any noise of thought and language floats. Although using a wide range of conflicting tools, methodological tensegrity is beyond dialectics proper in that it considers the oppositions of conceptual thought only a convenient manner of articulation within conceptual language - the actual duality a symbolic being can find itself in, the duality between language and silence - between every-thing and the void beyond description - is thus never entirely obfuscated. Tensegrity is therefore a triangulation between method and silence.

The methodological tools of this study are chosen by degree of departure from each other. Because experience caught in thought becomes inevitably herbalized, one way to resuscitate it is to compress it to explosion. Herbalized experience becomes a concept and concepts can float unobstructed between mediumatic layers, severing the energetic links between language and world. The deceptive capacity of language has been so disturbing not because, as Nietzsche 
claimed, there was an arbitrary value assigned to truth, but because misalignment means energetic loss, or, to use his terms - loss of power. Only in alignment with things, language can sustain those things and not float away from them, forever lost in a communication net. Alignment is a matter of relationships in depth, of layering, of multi-dimensional being - it demands precision and nuance. Unlike conceptual correspondences, it cannot be rounded up to the nearest whole number. The creative force of Ptah's words was not the force of concepts, but of living words uttered - language is the convergence of sound and concept, of body and form, even when it is committed to writing and its sound confined to mental reverberations. The concordance of sound and sign is the first level of alignment that a symbolic being must attain in order to have words resonate energetically (or "ring true" as the saying goes):

"Words expressed are not just symbols of mental states. The intent of the expression makes them objects of art. We may argue, then, that fine arts not only imitate men in action, as Aristotle would have it, they can also imitate words in action, the action of which may reveal character, thoughts and responses more than the meaning of the words.“" (Trott 1990, 93)

This semantic layering of symbolic expression is the concern of art - it shows the way art is concerned with truth. The emotion conveyed through the sound of the voice overwrites the meaning of the words so, if the two contradict, it is the emotion that is taken as the indicator of truth. The physical signs of emotion are much harder to control than their symbolic expression. This is the most simple first degree level where word loses its power. The art of an actor however is to master the nuanced control of vocal expression so that it aligns with symbolic expression and empowers it. Likewise, the art of the writer is to master the layering suggested in symbolic language itself - the nuanced alignment between syntax and rhythm, word and pause, continuity and disjunction. The tensions between positive and negative in language, in the sense of presence and absence (not in terms of opposing concepts), or the symbolic and what Kristeva calls "the 
semiotic" (3) reveal the cracks and holes that open to other layers - this is how tensegrity, contingent on the wriggle room that affords tensing, allows for constructing a work without sealing it off into an impregnable universe. A poetic work, an artwork, uses the limitations of a semantic layer to underline the possibilities of the semantic layer it is tensionally related to, adding multi-layered meaning to intensify that which is inexpressible by one layer alone. This optimization of the semantic potential of different layers of communication is crucial to art's impact. Thus, unlike scientific correctness, artistic alignment is not a relationship of confirmation in separation (e.g. a sad word pronounced with an illustratively matching sad expression), but of individuation in union - of a tensegrity between all the mediumatic layers each pushed to its untranslatable edge so that it contributes its unique angle through the tensional dynamics with the others. A sad word pronounced with a forced smile (a lie that betrays a truth about an extra layering of emotion and depth of character) would be an example of such tension that, although opposing the two layers to each other, arrives at a state of integrity by optimizing the polyvalent potential of each communication layer (e.g. verbal and body language). The cinematic moving image alone is a tensegrity structure balanced by the floating dynamic of image (colour, outline, contrast, texture), movement (rhythm, trajectory, direction), time (speed, duration), mediumatic texture (snow, pixel), light (intensity, flicker) and finally, editing which adds an entire new linguistic layer using all of the preceding ones as its structural elements. Thus methodological tensegrity reverberates with the compositional principles of art and the cinematic moving image, making it an adequate way of approaching the multi-layered entanglements that such compositions would inevitably present. Moreover, it allows for an aligned exploration of the four ways the moving image appears to us: the moving image as mental phenomenon, the moving image as technological medium, the moving image as language and the moving image as art. 
The methodological point of departure in this questioning was my practice as experimental filmmaker. I began with art-based methodologies that generated disclosure in the process of making as embodied questioning. These experiential findings pulled the focus on time and embodiment in the moving image. I began by questioning the pre-technological experiential modalities of the moving image, namely dream and memory (and more specifically, the dream memory). For several months I recorded my dreams and wrote down stream of consciousness memories monitoring the process through which the moving images within a dream and memory cohere and how could they be translated to or generate/externalize a moving image art work that necessarily would meet moving image technologies. I was interested in the ways the cognitive fabric of the pre-technological moving image converged/diverged from lived physical experience. How did the moving image assemble when unbound to the feedback of physicality, e.g. when a piece of furniture did not offer physical resistance to the movement of the dreamer (space) or when the movement from point $\mathrm{A}$ to point $\mathrm{B}$ is unbound to the protocol of physical/linear movement (time)? Who or what is the dreamer/viewer?

This process of introspection was intensified by academic reading and writing, a combination which combusted in the generation of several art projects, one of which, a moving image installation called The Eyes that Stop the Train, was carried out to completion (see appendix). Its production accompanied the first stage of writing this research and the two works reverberated off one another. In that installation I explore cinema in a range of moving image technologies, except film. A questioning of the mediumatic possibilities of those technologies both as an intimate experience and as discourse, it responds to Peter Kubelka's ideas about the irreducibility of the film medium with demonstrations of how digital medium has not only absorbed but also expanded the mechanical possibilities of the film medium in foregrounding cinema beyond film (and the respective new layer of mediumatic problems). Although the present 
research is intended as a stand-alone written work, the art project has been an integral part of my methodological process so, including its documentation as an appendix may shed additional light to some points and stabilize others.

The documentation of the art work communicates the ideas generated in the process of making but cannot provide for the communion that artistic engagement calls for. The work was deliberately designed to defy reproduction and documentation in ways that may exhaust or stand for the embodied experience. It intended to mobilize meaning as generated by the tensional alignment between language and experience. This sense of meaning actualizes and fulfils the core sense of meaning as form processed by thought - the two ends of meaning, minimal and optimal, represent the axis along which meaning oscillates between the simple and the complex, scientific reduction and artistic saturation. Even the simplest artwork, a blank canvas, a monochrome by Malevich or the controlled silence of John Cage's 4'33," mobilizes the tensional alignment between language and experience, and the openings to silence this alignment affords. Thus meaning is fulfilled through the tensegrity of language and experience, just as language is fulfilled in the tensions between perception and conception. Perception, in turn, is stabilized by the aligned pressures between the singular point of awareness (the mind-body) and the expanse that singularity is embedded in (the world). Therefore, embodiment is integral to methodological tensegrity and cannot be amputated from a discussion of the morphology of the moving image. Likewise, the first person point of view of Phenomenological questioning is indispensable in a questioning of a cognitive phenomenon not in the least because it provides the sole theoretical access to a candid perspective on human experience. The presumably objective position the third person affords runs the risk to become an outright obfuscation in a techno-scientifically dominated age. As a technique of interpersonal perceptual communion in art and mythopoetic thought, the third person has increasingly shifted toward a claim to a supra-human, divine, 
position when adopted as the sole optics of science. The advance of techno-science infused technology with the capacity of omniscience by liberating the human faculties from their embodied constraints - the camera eye, free from human limitations could roam and institute the third person as undisputed reality. While the mobility of the camera is an excellent correspondence tool, its perspective is no more true or free than the human first person one. The "objective" third person perspective became nothing more than the technological perspective - as such it could be called "objective" only in so far as it belongs to technological objects. This techno-scientific enframing of the third person obfuscates the original community connotations of the the literary device - the Homeric communion of sharing a witnessing:

"Consequently, the usual opposition of first-person vs. third-person accounts is misleading. It makes us forget that so-called third-person, objective accounts are done by a community of concrete people who are embodied in their social and natural world as much as first-person accounts. (Varela 1996, 340)

The nuanced range of experience and interpretation the first person makes possible is irreducible to objective rendering - a camera can capture a slice of what my eye sees but it can never capture the intensity with which my eye responds to the red of the chair or my mind dismisses half of the items that are in my field of focus. It can capture a close approximation of parts of our optical field, but it cannot capture the range of experience the act of seeing entails. In short, the camera can only capture that part of the visual experience that is accessible to and shared with its optics. Furthermore, a camera can communicate the items in this captured optical field, but cannot commune, i.e. It cannot provide a shared meaningful experience of that field unless it manipulates the image in ways that mimic the process of seeing (including the nuances of thought and attention that complete that process). In other words, it cannot commune unless it becomes a tool of the art of layering. Such an art-driven camera-eye would require keen 
observation of the process of awareness - a task exclusively available to the first person perspective.

"Experience is clearly a personal event, but that does not mean it is private, in the sense of some kind of isolated subject that is parachuted down onto a pre-given objective world. It is one of the most impressive discoveries of the phenomenological movement to have quickly realized that an investigation of the structure of human experience inevitably induces a shift to considering the several levels on which my consciousness is inextricably linked to those of others and the phenomenal world in an empathic mesh." (Varela 1996, 340)

Phenomenology is the scholarly approach that not only allows for the first person perspective to converse with the scientific third person but also, and most importantly, to unmask many of the artistic tactics that lace art with the dangers of mass indoctrination and propaganda. Unmasking the artistic techniques does not take the mystery out of art. Because the mystery has never been in the art itself but in what it reveals beyond itself - unmasking its techniques only demystifies art so that its lie remains true.

\section{NOTES TO CHAPTER 2:}

1. The examination of Biblical views cannot be overemphasized as the single major written reference of what would emerge as the contemporary Western culture within the worldview of which this work is written.

2. I repeatedly choose the word "afford" for its energetic connotations even when it sounds stylistically clumsy.

3. Kristeva's semiotic has evolved throughout her work, but here I am using the basic concept pregnant with the subsequent evolutions and honings. According to Kristeva, the semiotic is the other of the symbolic in language. It is non-representational, irreducible, belonging to "negative" space, "indifferent to language, enigmatic and feminine, this space underlying the written is rhythmic, unfettered, irreducible to its intelligible verbal translation; it is musical, anterior to judgement, but retrained by a single guarantee: syntax." (Kristeva 1984 [1974], 97) 


\section{Chapter 3. The Mind-Body Medium and the Cinematic Machine}

“Formerly — I don't know why — everything seemed veiled in a kind of mist. That is, I believe, because people think that the human brain is in the head. Nothing of the sort; it is carried by... the wind from the Caspian Sea." (Nikolai Gogol, Diary of a Madman)

Likewise, people think that the human mind is inside the head and that cinema is outside it. Nothing of the sort, in times of media technologies reigning supreme, the mind is carried by cinema - the wind of the sea blowing from inside the head. Bergson saw the brain as a kind of transistor channelling a universal mind expanse, a sea of sorts which makes Gogol's vision of a brain floating in the Caspian Sea, or any other sea for that matter, not that absurd after all. Especially when cinema enters the scene, laying claim on the function of the brain as the conjuring seat of the mind. But is cinema channelling the sea for us, is it channelling us for the sea or is it channelling us for us, or even more unsettling - is it channelling itself for us. To disentangle this conundrum, we shall look at cinema not as representation of photographed visibility, but as a furrow of a sea.

Bohm proposes that the universe is an "enfoldment" of light waves that contain all the information on everything in every part of space/time, reminiscent of the way a hologram contains information of the whole in each of its parts. The revealing or manifesting of any part of this universal content Bohm calls "unfoldment." The holomovement is the totality of this enfoldment and unfoldment of information which takes place in the movement of all fields, electronic, protonic, etc. (Bohm 1980, 225-226) This vision of the universe is not entirely dissimilar from Bergson's idea of the universe as an open totality of images. According to 
Bergson those images are neither embedded in empirical reality, nor contingent on subjective perceptual acts that format their appearance into the phenomenal world. Instead, they exist in a virtual state, in a state of infinite possibility, like a continuum of unactivated DNA code. (Bergson 1911, 1-3) While the term "images" (Latin for "picture," "statue," "copy") has arguable anthropocentric sensory connotations inserted into its universal claim, Bohm's emphasis on vibrational movement as the field of (the enfoldment-unfoldment of) information offers a more rigorous way to think of that which may imply the (humanly) unknowable. The vibrational movement, according to Bohm, encompasses the range of vibrational speeds with light waves setting the default speed of the holomovement. In other words, the holomovement is a physical phenomenon whereby form becomes a quality of speed - a view congruent with the earlier discussion of (quantum) form. Departing from a Bergsonian/Deleuzian view of the universe as an infinitude of images that she later relates to Bohmian unfoldment, Laura Marks provides a working definition of "information":

"Broadly, it is the set of images selected for their usefulness by particular interests. Information implies an interested viewpoint that gives form to the formless: a connotation that extends from the medieval scholastic Latin definition in the OED, 'the giving of a form or character to something' [Marks 2009, 88]

Ancient Greek has no word for information - the ancient Greek world is a world of form

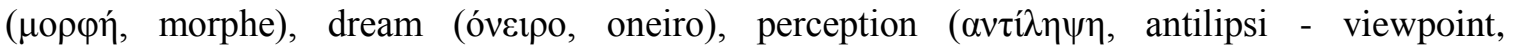
understanding) and knowledge ( $\gamma v \omega ́ \sigma \eta$, gnosis - cognition, memory). A form apprehended and retained becomes knowledge. The Latin root of information, informare, means "to shape, form" and also "to instruct, teach." Both the figurative meaning of the Latin word and Laura Marks' definition imply a cognitive process - a form that arises in the process of (necessarily in/ternalized) apprehension. It is as though the very touch of a singular point of view turns form 
into information. Such definition gives rise to the question, is information a strictly cognitive term or could we call it a pattern of data that can be (instrumentally) detected independently of subjective observation? How does a certain cluster of patterns from the "sea" of (perceivable) vibrations become significant to the perceiver, become information?

Marks defines her enfolding-unfolding aesthetics as a "manner of selecting what is significant" identifying significance as the cultural ways "to codify the perceptible, in order to discriminate in favour of those aspects of the world that are useful as information." (Marks 2009, 87-88) In other words, she is concerned with the process of privileging certain "images" from the Bergsonian expanse, by such acts as framing a part of the visible scape into a film image. This is an accurate account of enculturated significance, but an enculturated significance takes place in a field that is already a framed and ordered part of the Bohmian expanse identifying itself as everything (e.g. as the Bergsonian expanse) so that the choice is not as vast as Marx's implicit amalgamation of those suggests. In a more primordial sense, significance would mean the emergence of a pattern from the Bohmian field of vibration into an identifiable form. Thus the smallest identifiable component of the perceivable field would be significant by virtue of being identifiable, singled out. Semiotically, the smallest identifiable, i.e. sign/ificant, pattern would be a sign. A splash of colour or the shape of that splash could be signals, but an (identifiable) image is already a concept/percept reminiscent of the late Husserl's epistemic perception whereby any temporal, identifiable phenomenon emerges into consciousness through the joint process of assigning a concept and perceiving an entity. (Husserl 1948) When I see this cup, I don't have to process its components to "know" it is a cup - the recognition happens at the moment of (cultivated) perception, thanks to my capacity to extend my knowledge of cupdom to this instant. What this implies is that, from a phenomenological perspective, a field of images could only be called "of images" after it has actualized itself perceptually. An example of significance then 
could be the apprehension of a ball as a single object by a pre-verbal child who is playing with that ball. Clearly, the ball has certain sign/ificance with a variety of possible degrees of perceptual recognition, depending on the breath of actions the child initiates with it; however, it doesn't necessarily suggest the ball has yet acquired any (socialized) significance or meaning (as thought process) for the child. In the act of isolation/identification of a component from the field of vibration we are already privileging a certain way of engaging or "seeing," which then becomes the field of images from which we select and construct a plane of personal, social, cultural or political meaning. What Laura Marks seems to refer to by "significance" belongs to that already codified field of available signs or forms that Benjamin identifies in no uncertain Hegelian terms:

"It is not that what is past casts its light on what is present, or what is present its light on what is past; rather, image is that wherein what has been comes together in a flash with the now to form a constellation. In other words, image is dialectics at a standstill." (Benjamin 2002 [1940], 463)

Thus the image, in Benjamin's terms is, rather than a potential code, an optimal manifestation of the passage of time - an enfoldment of time into an extra dimension that provides an instantaneous glimpse at that which from the lower dimensional plane can only be encompassed with the passage of time:

"...while the relation of the present to the past is purely temporal, continuous one, the relation of what-has-been to the now is dialectical: is not progression but image, leap-like or crack-like.” (Ibid.)

Benjamin's image is a poetic phenomenon formed by the dialectical pressures of time that break and penetrate the wall of continuity. This emergence of form from the curvature of time is analogous to the way the three-dimensional perspective can allow the onlooker to instantly perceive a cube while the citizen of Flatland in John Abbot's book of the same name can only 
experience the cube as a continuous line by walking along it in time. (Abbot 2002 [1884]) Thus, what is only a time-based projection in Flatland becomes a fully rounded body in our threedimensional world. This energetic difference between time as flat continuity and time as tension informs Benjamin's idea of image authenticity and its embeddedness in language:

"Only dialectical images are genuine images (that is, not archaic); and the place where one encounters them is language. Awakening." (Ibid.)

Benjamin's view of visual language as coalescing under the dialectic pressures of time, by dramatizing the Kantian schemata (the interaction between imagination and time to enable the actualization of a priori concepts into a posteriori ones), foregrounds temporality as an active force in the axis form-language that can be extended to the axis vibration-form.

Before we register phenomena on our horizon, we ground them in a field that secures the registry itself. It is the field that both makes possible and makes exclusive the experience of a reality as though it is available directly to us. This perceived directness demands a more radical examination of how information arises at the least mediated possible layer of observable cognitive engagement, how does it set itself to be unquestionable or "real." The demand for a least mediated layer converses with Marshall McLuhan's idea that the "content of any medium is always another medium." [McLuhan 1964, 23] In order for anything observable or detectable, i.e. formed, to be brought forth, it has to be mediated - these words have been mediated through writing and writing is mediated either electronically or by print. Let's assume, you are reading this in print - it is rendered visible by light, light is mediated by the sun's plasma or by a light bulb's electricity, as the case may be. The sun, however, is not precisely mediated by another entity-like medium. Rather, it is brought forth by thermodynamic processes, not unlike Benjamin's linguistic image burst out of temporal combustion. Therefore, the sun is the last observable mediumatic entity in the line we are following. The relationship of the sun to sun-light is the least mediated 
layer of visibility of this writing, providing you are reading in the day light. Conversely, to find the least mediated layer of observable cognitive engagement, we follow from the written word as mediating language, through language itself mediating a thought, and finally, thought which was mediated by my body. Thus, the body is the least mediated observable layer of cognitive engagement. As far as we corroborate (a) reality that makes this text intelligible, embodiment is the seat of both our cognition and our action. If we accept this line of organizing mediumship, no observable cognitive engagement could be articulated as fully unmediated.

The cognitive/sensory consolidation of the moving image into a coherent language eventually becomes technologically available through cinema. Because of the layering of this consolidation, the moving image has proven a powerful tool for bypassing critical filters, a hypnotic tool of both propaganda and, more primarily, of cognitive conditioning. This is why, to understand the impact of cinema, it is crucial to examine how thought is embedded in the prereflective sensory, and how it comes back to us through the reflection of cinema. How can we understand the conversion of data into information as a sensual process (the holomovement as a sensual phenomenon in so far as it signifies nothing yet)?

\section{Shimmering Thought (on the Screen of the Mind)}

According to Robert Logan, language is a complex system that emerges and evolves as an organism driven toward unification - identifying and synthesizing individual patterns into lightweight concepts that can encapsulate a potentially infinite amount of information, e.g. the concept of tables includes all possible instances of tables (plus an infinite set of tables). (Logan 2007) This view of language, concerned with the life of language driven by conceptualization, leaves untouched the question of lived language: 
"Because meaning is the total movement of speech, our thought crawls along in language. Yet for the same reason, our thought moves through language as a gesture goes beyond the individual points of its passage. At the very moment language fills our mind up to the top without leaving the smallest place for thought not taken into its vibration, and exactly to the extent that we abandon ourselves to it, it passes beyond the signs" toward their meaning." (MerleauPonty 1964, 43)

This vision of language as the force that slows down and overloads thought enabling it to generate meaning points at the stabilizing effect of language to thought as instrumental in the emergence of meaning in thought processing. In Chapter 1, we arrived at meaning as "not only the passing of information through thought, the internalizing of form, the making of flesh into thought so to speak, but also the passing of thought through form, the lived experience of thought, the making of thought into flesh.” (p. 27-28) So, on its way out, language speeds up and unifies, as noted by Logan, but on its way in, as a learned, heard read or thought information, i.e. in apprehension, language slows down and fragments (Merleau-Ponty). In speech and writing language tends to be coherent, lightweight and fast, in thought, it is fragmented, heavy and slow. To adjust to the mediation of thought, so to speak, language has to go back from concept to precept with the respective breakdown of symbols to signals.

Does that mean that language is energized by or only energizes this twofold movement of thought and form? According to Logan language is an emergent phenomenon defined by an autocatalytic process whereby words and concepts "bootstrap themselves into existence." (Logan $2007,45)$ He describes this process of language emergence as the convergence of information quantitative (over)flow into a new single quality:

"A word operating as a concept acts as an attractor for all of the precepts associated with that word. An attractor is a trajectory in phase space towards which all of the trajectories of a non-linear dynamic system are attracted... The attractor is a strange attractor because the meaning of a word never exactly repeats itself. The trajectories of a strange attractor never meet, even though they come infinitesimally close to each other. It is the same with a word. The meaning of a word fluctuates about the strange attractor, but it is never exactly the same 
because the context in which the word is being used is always different." (Logan 2007, 49-50)

A word in flight, a concept, could therefore be thought of as a kind of magnet that, when pulled by the gravity of the embodied mind, slows down and, in turn, stabilizes the mind-body itself. Concepts attract an infinite range of infinitesimally close but not overlapping meanings $-\mathrm{a}$ virtually endless supply that breaks down when that same attractor is attracted by a singular embodied instant, a thinking individual. The overflow of words thus grounded generates meaning that then floats toward a concept, and the cycle goes on. This animation sequence foregrounds not only the tensegrity between Logan's and Merleau-Ponty's takes on language, but also the entanglement of concept and embodiment in language, a way language distances (from each other), intertwines and unites mass and form, body and thought, knowledge and communication.

The moving image as a faculty of thought is therefore subject to the question of language of embodied knowledge. The English word "knowledge" has overtaken most of the colloquial functions of "cognition" so that the two English words do not provide an equivalent to the way French actively distinguishes between connaitre (holding personal knowledge, experiencing knowledge) and savoire (having instrumental knowledge, idea, access to/use of knowledge). Similarly, the Greek gnosis (personal knowing) and eidein (general knowledge, also the etymological root of "idea") refer to embodied cognitive processes as distinct from conceptual shared knowledge; as does the Latin agnito (knowledge as recognition) and conscientia (conscience, consciousness). The relationship between idea and consciousness, instrumental to the understanding of conceptual language as a lightweight strange attractor that promotes distancing and free mobility, becomes explicit in the axis eidein-conscientia which describes impersonal knowledge in terms of the disembodiment of an idea and the distancing power of consciousness. Conceptual language can thus be thought of as the projection of the Bohmian 
holomovement as a DNA-like continuum (akin to Bergson's sea of images) that cuts through embodiment but can be accessed through the distancing of consciousness and grounded in the body which acts like a mediumatic transmitter, congruent with Bergson's understanding of the brain [Bergson, 1911, pp. 19-20]. The third Latin word for knowledge scientia, etymologically rooted in the PIE skei-, meaning "to cut, to split" makes science the obvious successor of conceptual language as the part of language that separates and abstracts. It is this axis of communicable knowledge that also accommodates the French savoire (from Latin sapere, to taste) as the instrumental, empirical way of knowing as skill.

Bergson's sea of images interfaced by the brain is modelled in contrast to Kant's idea that the mind possesses a conceptual matrix that he called a priori concepts and contrasted to the empirically derived generalized abstractions called a posteriori concepts. Thus, according to Kant, concepts as abstractions are not emergent, as Logan would have it, but secondary to and enabled by a (DNA-like) formal blueprint encrypted in the human mind. (Kant 1988 [1800]). This reverberates in Humboldt's idea of universal grammar suggesting that "underlying any human language we will find a system that is universal, that simply expresses man's unique intellectual attributes." (Chomsky 2006, 67) This cognitive entanglement convinced Humboldt to conclude:

"...language is not really learned - certainly not taught - but rather develops "from within," in an essentially predetermined way, when the appropriate environmental conditions exist. One cannot really teach a first language... but can only "provide the thread along which it will develop of its own accord," by processes more like maturation than learning." (Chomsky 2006, 67)

The structure of the process of linguistic unfoldment from linguistic blueprint to fullblown empirically grounded language Chomsky called generative grammar and conceived in response to a quantitative gap between input and output in the learning process: 
"I think that if we contemplate the classical problem of psychology, that of accounting for human knowledge, we cannot avoid being struck by the enormous disparity between knowledge and experience - in the case of language, between the generative grammar that expresses the linguistic competence of the native speaker and the meagre and degenerate data on the basis of which he has constructed this grammar for himself." (Chomsky 2006, 68-69)

Thus while the rationalist models of the movement concept-language try to account logically for poetic leaps in the cognitive process, empirical models such as Maturana's use the autopoetic principle to trace the logic of language emergence, without much of the confusion anticipated by Chomsky:

"There seems to be no substance to the view that human language is simply a more complex instance of something to be found elsewhere in the animal world. This poses a problem for the biologist, since, if true, it is an example of true "emergence" - the appearance of a qualitatively different phenomenon at a specific stage of complexity of organization." (Chomsky 2006, 62)

However, as Maturana's theory demonstrates, the biologist comfortable with the nonlinear principle of autopoiesis, as manifest throughout the living world as the unfoldment of DNA, has no challenges in transferring that principle to language. Likewise, the quantitative principle that Chomsky summons to argue generative grammar is a native of physics and chemistry. Conversely Logan, a physicist, builds an empirical theory of linguistic emergence informed by his work in the physics of complexity. Both rationalist and empiricist models of language have to account for linguistic poiesis, not only as a form of language but as a principle governing language. However, both empiricism and rationalism belong to the same order of reason, and reason as a sequential way of ordering cannot genuinely account for poetry. It can only open a space for poetry to reveal itself, or else it would close into a moebius circuit where any attempt at locating a point of beginning can only be a designation, a manner of speaking. 
Chomsky's reasoning about cognitive processes is grounded on the physical principle that no matter is ever lost, only transformed. This morphology of matter however cannot be applied automatically and without scrutiny to a morphology of form (which is what language is - form without matter). This formal nature of language as a cognitive phenomenon has emboldened a rationalist abstract understanding of language as a conduit of knowledge.

English language discursive constructs tend to avoid considering knowledge that is not consciously accountable for. When, for instance, a baby starts walking, we tend to say "the baby started walking" instead of "the baby learned to walk." It is the moment of the first independent steps that is celebrated, as though it occurred by a miracle and not through a process of trial and error, of learning that is. To say that someone learned how to walk implies that s/he had a possible physical impediment that required conscious effort to achieve what babies seem to achieve automatically. Bergson gives a simple (and naively unselfconscious about its underlying assumptions) reasoning behind the dismissal of the possibility that somatic behaviour could signify knowledge - to demonstrate that the brain and nervous system are not the seat of knowledge, he points out that all lower organisms exhibit somatic behaviour mediated by a nervous system yet they do not possess knowledge. (Bergson 1911, 19-20). The latter implies that cognition is contingent on epistemology - an axiomatic given dominating Western thought since Plato (and challenged by Chomsky's observation about the emergent nature of language). This position is representative of centuries of hierarchical denaturalization of humanness promoting the idea of knowledge as uniquely symbolic/conscious and therefore exclusively human. Without going further into the ideology of such a positioning, a reductive thought experiment in a baby's first experiences may suffice to open up the concept beyond socio-historical confinements.

Equipped with no language, mobility or fully developed perceptual apparatus, it is highly probable that a newborn distinguishes little of the mesh of stimuli s/he is exposed to. As the 
caregivers begin to repeatedly prioritize information, such as the voice of the mother, they mobilize the baby's ability to focus, i.e. to isolate patterns of information from the mesh of data as stimuli. This act of summoning the baby's attention into focus marks the beginning of the cognitive process. Mobilized by the environment (caregivers, chance occurrences, etc.), the sensory apparatus begins to slowly adjusts and "learn" to interpret information in ways that are later conceptualized as representational or more colloquially, as visual, auditory, tactile, etc. This gradual process of the body aligning in a certain way (sensorial, motor) to the broadcast of the environment is a learning process that meets the most general sense of the word "knowing" as acquiring information, including the instrumental knowing-how (savoir). In this process the baby learns how to isolate patterns and eventually, how to use those patterns to consolidate itself as both separate from and continuous to its environment. In other words, from the first sensory focus (auditory, visual or tactile, etc. as the case may be), the baby begins to assemble principles that would gradually cohere the physical world.

The conceptual human has naturalized the process of this initial gathering of the body into the world, and its dual articulation (inside-outside). Thus when Bergson talks about a universe of images, he is already talking of a conceptual universe, a (possible) universe filtered through our capacity to articulate it. In that universe, the brain floating in the Caspian Sea is a given that only needs to be brought forth by privileged attention. But how these images, blueprints, holograms or a priori concepts, as the case may be, immanent as they may be, are nonetheless sensually consolidated. What, if anything, underpins their organizational order and makes them potentially identifiable?

The spatial naturalization begins perhaps as early as the first scream into the world, with the first unfamiliar touch of dry air permeating everything outside the mother's womb. However, underneath our conceptual articulation of that process as the passage from an "inside" into an 
"outside", there must be the experience of difference as a variation of vibration, akin to the vibrational difference on my skin when I close my eyes, shut my mind and feel the water as I float on top of the sea and the dry air that floats over me. The foregrounding of this vibration-assensation is a necessary step on the way to a more primary understanding of knowledge that would account not only for embodiment, but also for the way this embodiment is enmeshed with knowledge, or rather, the way the body is assembled by its knowingness.

When I stop writing and start observing my thought process, I notice I hold no single clear thought at any given moment. As I look through the window, a myriad of sensations stream simultaneously through my mind. But this is not an uninterrupted flow. Rather, they fire up in momentary flickers, sort of end-tails or abbreviations of forms, neither fully visual or sonic, nor fully conceptual-linguistic. Those snippets are not formed entities but only references or directions ("trajectories" as Logan would call them). They point to thoughts, ideas, connections. This remains valid even when the mental chatter is minimized to sensory experiences: when my attention is drawn to a singular sensory focus, it is still embedded in a field of snippets of sensory information that shimmer, albeit less intensely, in the background of my perceptual-mental "screen". In other words, this experience is accounted for both physically (as far as I am accounting for my sensory enmeshment with the world), and mentally (as far as I am accounting for the thought process during a minimized sensory state, e.g. if I closed my eyes in a dark anechoic chamber). In search of the ontic way of holding "information," we found ourselves, or rather the self as a point of focal convergence, floating in the shimmer of thought. This shimmer of thought seems to unevenly correspond to Bergson's universe of possible images although, unlike the latter, which expands beyond the actual into the infinite virtual, shimmering thought is already actualized in the self's unfocused awareness. It is the world-body availing itself to itself, so to speak. In that inverted expanse, the brain could indeed be floating in the Caspian sea, re- 
mediating itself as a medium. This is not entirely remote from Bergson's own vision of the brain as medium, as "nothing more than telephonic exchange," [Bergson, 1911, p.19] neither it is entirely unlike Metzinger's ego tunnel where the world is an inverted expanse of neurological representations [Metzinger, 2009]. However, the brain, if we are to pull it into focus from the shimmering expanse and investigate its position, is not only a physical medium floating in a sea of possible articulations, neither only a point of instrumental convergence within a representational expanse of its own articulation, but a vibratory pattern floating through a sea of (physical) vibration that is at once floating through it. It could be said that, in this shimmering knot, any focal movement, or cognitive attempt, is referential, in the sense that it refers to something more, it is an end tail, not a complete unit of information.

The referential quality of lived (as distinct from encoded) knowledge shifts the theoretical ground from static-spatial metaphoric departures to temporal-energetic ones. Shimmering knowledge, i.e. knowledge as vibration, has no steady beam yet is there steadily, it is there yet always already in flux. Once again, prioritizing a neuroscientific perspective, Metzinger offers a closely approximate, but not coinciding, vision of the world as somatically mediated vibrational expanse:

"The whole idea of potentially being directly in touch with reality is a sort of romantic folklore; we know the world only by using representations...” (Metzinger 2009, 9)

This would be entirely valid, if the world were exclusively external to "us", if we adhered to the representational designation of "here and there", of "inside and out" as final truth and not just a manner of speaking. But such exclusive positioning of the conceptual description of the world is a logical impossibility since, by its own understanding of knowledge and finitude, we cannot know what is or is not beyond that tunnel of representation and, therefore cannot genuinely claim a final position. An articulation of the world as external could be made possible 
only when the vibrational quality of the world is stabilized into an immovable ground, on which concepts begin to stand for reality. This is why, while the following clarification may be orienting in the ways scientific perspective converges with the current questioning, it can only be read in brackets, as the metaphoric conceptualization of an intricate process that conjures said "world" out of an unknown expanse of vibration:

"The world is not inhabited by coloured objects... It is just as your physics teacher at high school told you: Out there, in front of your eyes, there is just an ocean of electromagnetic radiation, a wild and raging mixture of different wavelengths. Most of them are invisible to you and can never become part of your conscious model of reality. What is really happening is that the visual system in your brain is drilling a tunnel through this inconceivably rich physical environment and in the process is painting the tunnel walls in various shades of colour. Phenomenal colour. Appearance. For your eyes only." (Metzinger 2009, 20)

This "ocean of... different wavelengths," a layer of the holomovement that we not only access and harness through modern technologies as an expanse of potentially translatable frequencies, i.e. and expanse of potentiality that gives rise of the formal layer we articulate as sensible reality, but we can also access sensually through the intimation of the texture of meditative silence or the flicker of light. In other words, the expanse of wavelengths we have tapped into scientifically, an expanse of continuous data harnessed by out technologies, permeates the known as the shimmering knowledge that a ripple of attention assembles into a body-world, or what Metzinger alludes to as the ego tunnel. In other words, from the wavelengths of energy (light as electromagnetic radiation) to the shimmer of form (light as elementary particles), the vibration of those primordial fields can be felt behind every consolidated sensory experience; those vibrational fields bring the world to us not as a specific concept or perception but as an all permeating feeling.

Thus shimmering knowledge, as that which animates shimmering thought, is a phenomenally grounded view that describes the fluid condition for the emergence of the tangible 
physical and the thinkable symbolic that make eidein and gnosis emerge, converge and diverge all at once. This view accounts for knowledge in the process of cognitive gathering as enactment whereby holding knowledge is itself an act of assembling reality, not merely a passive collection of symbolic capital in a mental library. This active view of knowledge, knowledge as a lived experience of the world-body, focuses on the vibrational directness of the cognitive process, and not only on its symbolic mediatedness, as has been traditionally the case. Shimmering knowledge is the pulsation of information felt directly in the body-mind as it focuses the body-world into thought. It is the membrane of metabolic exchange between the singular attention and the cognitive reflection of the world in the singular attention. In short, it is the field where shimmering thought emerges.

A phenomenological description, inevitably singular and subjective, therefore exploring what is possible rather than what is statistically probable, neurologically observable, etc. may best articulate the experience of this field. But first, let's backtrack to our thought experiment.

The new born is "thrown" (Heidegger) into a world that feels entirely different from the feeling of being one with(in) another (organism). This feeling of being, while floating in the mother's womb, must be senseless and timeless. It has, at best, a tactile character but, since that tactile texture is all-encompassing (the amniotic fluid encompassing the baby's body) it is not distinguishable from the feeling of being itself. In terms of time, it is inverted, durational and processional as all the unfolds is the baby's own embryonic development. It is an eternal moment of incrementally unfolding being, pulsating with the rhythm of the expanse it is one with. When the mother's body begins to reject the baby, it initiates the first disturbance of that state - it is the beginning of action and sense being. Until now, it has only probably received signals but now a signal becomes an unconquerable force - the baby's body receives the first push to move (out) - a pressure from and to what would henceforth gradually and consistently delineate itself as the 
"outside." This first spatial demarcation is thus the inauguration of the mind - the place where time and space begin to emerge. The baby is expelled from the ambiance of the mother's heartbeat to a world of rhythmical cacophony, of chaos and action. Could the clock - the techno-scientific search for measure - be an attempt to recapture that original rhythm of the mother's heart?

Once "outside," the singular point of awareness (the baby) is bombarded with a myriad of unfamiliar sensations triggered by the vibrating dryness of the air, the onslaught of flickering light, the pulsations of acoustic sounds not buffered by another body. Of course, the newborn does not distinguish, let alone recognize any of these, neither does it isolate different sensations they come as a cacophony of unfamiliar vibratory feeling of being somewhere else. Since sound is more forceful than sight (a single sound can pierce the sensual carpet and summon auditory attention, while the visual prioritization depends more on our choice of focus) it is plausible to imagine that the first outside signal the baby isolates, after the initial mess of tactile sensations, is a voice. Indeed, obstetrics has long accepted that sonic familiarization begins in the womb. But while in the womb, the mother's voice is part of the baby's body-world, its externalization must be an entirely new sensation, both disturbing and comforting as it throws a rope between the familiar and the alien. With that first focus into that new state of being, the baby is called upon to isolate a pattern - the voice - and pull it out of the perceptual mesh. Thus begins the directed assembling of a possible world that is necessarily a world-body - an extension of the embryonic beingness in flesh. The baby is mobilized and starts mobilizing the vibratory carpet into sensory signals or percepts (the voice). It has been given the first prompt to focus and has achieved the first act of attention toward interpreting. This is the initiation into an assemblage of a world as a sensory mesh, that would eventually, with the isolation of enough vibratory patterns, begin to mobilize matter into meaning, and language. 
The baby is given no choice - from the first call into this world, its attention is guided into a certain way of assembling the world, a particular sensible way to consolidate it as physical. However, the first painful separation from the mother's body is also the first gift to the baby. The gift of separation has forced and violated the baby, but it has also allowed it to now expand and take over the world. The danger of fragmentation and annihilation is also the promise of knowledge and freedom. The baby has been expelled and amputated from the body of its world but it has been given a gift with which to navigate and assemble a new world that it can only be expelled from by death. From a boundless blob of vibratory feelings, the baby, under the focusguiding pressure of the already-assembled world of its caregivers, gradually becomes a singular point of assembly of the bound body-world it constitutes. A world that the baby, now armed with the gift of separation, has to get to know not by storing information but as an act of reuniting. The baby is challenged to find out how far it can chop up the world to incorporate it without annihilating it. It is beckoned to embark on at once reuniting with the world and on escaping it.

I close my eyes and let my mind relax into itself - I focus on the sensations in my body and the darkness in my visual field. As the dialogical activity of my wakeful body-mind slows down, I feel a myriad of snippets that shimmer in my field of experience. I don't see, hear or even think them, although some of them are sonic, some visual and some quasi-conceptual - I feel them as they happen. Like a strangely textured carpet, they underline my entire feeling of being here now. There is no particular line of verbal or image-based thought that holds them together, and even when I pull my mental focus on a thought, the snippets remain flickering in the background, always ready to pull that thought back into the shimmering field. If I decide to focus on an element of the carpet, I seem to be isolating a pattern - for instance, I just isolated a fluffy line of orange glow which floated in place, always ever so slightly changing intensity and outlines, as long as I kept my eyes' attention on it. This was a percept that might become a cluster 
of concepts - "a fluffy line of orange glow." The "fluffy line of orange glow" is a heavier package of information than the actual experience of the percept that I carry in my thought.

It took me about a second to focus on the percept, to pull it out of the shimmering field already a considerable time/energy investment compared to my simply carrying the shimmer. Moreover, it took me a few minutes to figure out a linguistic way to describe it - an even heavier, time/energy expense. This is an example of how language departs from thought by gathering mass, so to speak, or more precisely, by gathering time and space. The heavier the language, the more meaningful, the more it is articulate and detached from the carpet of shimmering thought congealing snippets of the latter into thought that "crawls along in language" and yet at the same time "moves through language as a gesture goes beyond the individual points of its passage." (Merleau-Ponty 1964, 43)

As I close my eyes and let my mind relax into itself, I open up to a certain experiential plane that is available to me when I relinquish control over my inner focus. In other words, it happens on a particular plane of attention, or gathering. This experiential plane has no sensory focus, no processing priorities, it is only a vaguely articulated field of feeling. From the position of someone who has already assembled the body-world, I can only articulate that field within the sensory parameters of the experience. So, the vaguely articulated field of feeling that emerges when I calm down my body-mind and let it float into itself, in articulation, comes into semantic focus as a field of pulsations.

As it happens, I can emerge from that field and gather another layer, the layer of perceptual differentiation. If I pull my visual focus with eyes closed, I begin to see a coloured floating shapes shifting in my field of vision. But there is no one way to witness this picture as attention and focus intertwine - I can sustain the picture and stay with it mentally and visually, or I can sustain the picture visually and mentally wander away from it as it remains constant in my 
field of vision. In addition, I can play with deep and shallow visual focus that gives me slightly different pictures or, with controlled and uncontrolled focus whereby I can either allow the picture to float any which way or I can attach my attention to it so that the latter restricts, slows down or directs the picture's movement. This shifting focus is what allows me to solidify the initial shimmer into pre-conceptual/perceptual information (e.g. the floating visual field) which then can be sustained as a perceptual/conceptual information (e.g. a sustained picture of colourful stripes and morphing circles) which then becomes symbolically manageable, i.e. possible to transfigure into another mediumatic layer. It is the focus on the colourful shapes that allows me to resonate with the world of physical bodies. But only when I step into the symbolic, I can articulate those bodies as identities that can therefore become concepts. This architectonic layering of interpretation is flattened in language, and the experience of the shimmer - obfuscated by the foregrounding of symbols.

Thus the focus is the thing given to a baby on its way to join the shared reality of its caregivers. It is the first item in the toolkit of the gift of separation. The shifting focus (of attention) is arguably the first skill the baby can master, the first instrumental knowledge.

As already discussed, it is quite a task, both futile and cumbersome, to articulate symbolically to myself, i.e. to think in words or pictures as the latter both slow down the thinking process and minimize its agility, making thought either "crawl." In the way language slows down and stabilizes thought, language is to thought what perception is to dream - a fleeting reference that both fills the "content" of thought, makes it possible to apprehend, so to speak, and at the same time resists it and yields to it. Language is thus both alien to and native to thought, just as perception is both alien to and native to dream. We need our sensory memory to experience dream and yet our senses are shut down or minimized in sleep. 
The linearity and precision of language is akin to physicality in its organization on syntax, on a system of laws that make it alive, make it a world. Likewise, physical reality rests upon a set of laws which, like a syntax, sustain the integrity of that reality - the table resists my presence by being a consolidated presence of its own that can yield to my gaze or touch only if I abide by certain linear/directional rules of physical interaction (e.g. I move around it, directionally, in order to pass on the other side, I apply a certain amount of pressure in order to move it). It is to its consistent solidity, to its resistance to my gaze and touch, that I owe the "reality" of my perception and the respective precision of the word "table" (which is nonarbitrary in the sense that it consistently refers to the same class of objects and the same type of things). But when I close my eyes and think of this table, no symbols resonate in me - rather, there are snippets of something quasi-visual, quasi-conceptual that refers to the table, to its position in space - a position I feel in my body as a perceptual residue - a kind of floating amorphous referent that solidifies only if I either recall the image of the table (which emerges uncertain but following the photographic outlines of my perception) or if I pronounce the word in my mind. If I try to think of tables in general, there emerge some geometrical snippets in my mind that, upon pulling my focus, solidify into a vague slice of a small part of my living room where I can physically approximate (as a feeling in my body) the edge of my coffee table. This random and unstable picture/feeling is what currently refers me to or is referred to the concept of tables. I am certain that it has changed many times as elements in this particular picture/feeling that I just described is only a relatively recent event in my life while I've known the word table for years. Thus, by writing about the "table" I invoke Bergsonian linear time, upheld by my memory of the concept of table and all my experiences of tables, and I merge it with Logan's language packaging whereby the assemblage of the concept "table" projects my future 
recognition of yet unknown and unseen, untouched, un-thought and unremembered tables. As far as it consolidates a continuity, it projects a future memory, to use Bergsonian language.

My association of the phenomenon of table with the word table is an amalgamation of immanence (physicality) and abstraction (symbolism) that, although foregrounding the latter, is always already contingent on the former and vice versa (as an exemplary matter of the shifting focus). As I pronounce the word "table" in my mind, my thinking process slows down and begins to feel the resistance of something imposed - I just imposed physicality on it because the word is an emergent from a specific physical action (vocalizing) - I had learned the word by physically enacting (pronouncing) it in a particular language (in this case, English as my second language). Moreover, being multilingual, when I think of tables, I don't think in any specific language. I begin to think in a specific language the moment I begin to think of table in a language-specific context, e.g. as I am writing those sentences in English. Pronouncing the word mentally is a learned skill acquired twice - once with the spoken word (when, right after beginning to talk and continuously vocalize their observations, kids find out they could think in words or word-snippets without pronouncing them) and once with the written word (when they learn, as part of any school curriculum, to read without eventually vocalizing). In both cases, in-corporating language as an inner experience rather than a performed one, speeds up its processing by allowing for the minimization of physical involvement in "snippetting" so to speak. Thus the body-mind while fluent at generating symbolic language as ex-pressions, does not tolerate them well as impressions so much so that it has to break them down to pre-concept-type entities again, or more precisely post-cepts. Thus, snippeting deals with lightweight post-ceptual information that requires little energy and therefore allows for much faster processing (e.g. when I read these lines, I don't read them letter by letter but perceive words and even phrases as instant entities that my attention glides over). 
In short - words as a posteriori concepts, i.e. as abstracted from physicality could only exist as embodiments of those abstracted concepts. From snippetting to pronouncing to writing, we can only talk of certain embodied manifestations of concepts. Those embodiments are necessarily metonymic and referential to both the general concept, and its embodied instances. This is demonstrable both by early childhood language development and by adult use of spoken language, as noted in Merleau-Ponty's notion of language as (metonymically) contained in its components:

"It has long long been known that for a child the word first functions as a sentence, and perhaps even certain phonemes as words." [Merleau-Ponty, 1964, p. 241]

A statement congruent with Chomsky's generative grammar, yet by itself revealing the semantic potency of linguistic fragments when they emerge in an already consolidated sensorium. Chomsky's quantitative discrepancy of language learning would be a genuine mystery if it occurred in a cognitive vacuum, but it always already occurs in a socio-cultural context where language acts as a cognitive layer transliterating an already consolidated sensory realm necessarily organized by syntax. And syntax, as any set of rules, can produce potentially infinite variations of a finite input.

The second item in the toolkit of separation is repetition which becomes a rule long before the child steps into symbolic language. But the rule, by asserting the focus, inaugurates a movement back to unification, to holding together the things separated in space. Thus, when the symbolic language arrives, the word that is given to the child to galvanize the separation of things is always already imbued with the power of the rule - to unite the separated. Moreover, that word arrives in a place already assembled - it arrives within a syntax, i.e. within a sentence.

Shimmering knowledge foregrounds the field of perceptibility from which an element is pulled into focus, initiating its pre-ceptual emergence into an entity with its own boundaries. The 
conceptual assembling begins with the identification of that element as a singular pattern and completes its trajectory when this pattern is identified as a pattern reducible to a sub-pattern that organizes similar phenomena into a group of entities. Therefore, mental snippeting is (based on) a memory of first perceptions. It foregrounds primary somatic knowledge over symbolic knowledge and thus makes embodiment the demonstrable field of the cognitive process. The default state of the mind-body is not a thoughtful state but a state as close to formlessness as the operating syntax would allow. As language becomes incorporated, this state is flooded with articulations that gradually get trapped by the focus of attention and become naturalized as specific thoughts.

"With regard to the superstitions of logicians, I shall never tire of emphasizing a small... fact, which is unwillingly recognized by these credulous minds--namely, that a thought comes when "it" wishes, and not when "I" wish; so that it is a perversion of the facts of the case to say that the subject "I" is the condition of the predicate "think." (Nietzsche 2003 [1886], 17)

Indeed, the discipline of thinking is not only secondary to the state of free-floating articulations, but even its inception by the focused entrapment of articulations more often than not leads to the involuntary retention of thoughts and not to a discipline of thinking. Interestingly, the latter can only be claimed in exercises akin to phenomenological reduction, i.e. to bracketing or letting go of thoughts and returning as close as possible to the shimmering screen. Thus Nietzsche is correct as far as thoughts as distinct units of consciousness are concerned - but those thoughts are a movement summoned by the gravitational pull of the shimmering field which is both thought-like and knowledge-like, and are actualized by the formal snippets already floating in and filling that field up. It is those snippets that are mobilized into actual formed thought by whatever incites the shimmer. Thus the shimmering field is always already there, impersonal and nonvolitional, yet pregnant with the possibility to emerge into thought and knowledge upon the slightest stimuli and/or shift of focus. 
The shimmering field permeates everything and acts as the metabolic screen of the mind, the plane of though. It makes possible the emergence of the mind as that which is thinkable. The mind is a description that a particular way of focusing makes possible, solidifying form in one version of reality. Thus the shimmering field could be likened to the embryonic fluid of knowledge. We experience only snippets and shimmers of the information we articulate as knowledge, with a vast part submerged in the embryonic fluid. Thus the shimmer is a shimmer of knowledge that can revive the description into meaningful articulations. The description is inert, only the shimmer is "alive," in flow. Thus the shimmer as the screen of thought is a way to talk about the fabric of predicative experience that conditions linguistic judgement as per Husserl's idea of judgement formation (Husserl 1974, 20).

The intricacies of the cognitive process make it both vastly expandable and hospitable to a range of theories and observations, and at the same time ultimately immeasurable and therefore technologically inaccessible despite the headway of neuroscience and modern technology. Its understanding is thus contingent on a trans-disciplinary approach, where cinema and technologies of the moving image can be of instrumental value. How does this thought-world interplay appear in our experience of the three modalities of moving image - dream, wakefulness and cinema? Or rather - how does it come to us in cinema as dream and cinema as reality?

\section{Cinema}

Cinema comes to us like thought, but behaves like reality. Perception learns to adjust to the mediumatic layers of reality - when I was a child I believed, like many other children, that the people on TV were just like the ones on the theatre stage, only trapped in the TV box. Eventually, I learned to distinguish between the textures of the physical and TV broadcast. Likewise, early 
cinema viewers are reported to have experienced cinema more vividly than viewers a hundred years later. Conceptual learning about the conditions of an image trains perception to identify not only the environmental context of an image but also its signature within the image, i.e. the image's mediumatic environment. The 21st century Western human, through constant exposure to a range of moving image media, tends to automatically distinguish between the film grain, the video grain and the "grain" of lived "reality." In moving image media, more than in any other visual experience, the visible grain of the picture, the shimmer of its texture so to speak, reveals instantly to the unarmed eye the physical medium that channels it. A "snow" of a magnetic video is not only known but is also experienced, in the distinctness of its shimmer, to be qualitatively different from the dots of a $16 \mathrm{~mm}$ film or the pixels of a digital video:

"With $8 \mathrm{~mm}$, a leaf on a tree will be made up of maybe four grains. So it's very impressionistic, almost like Seurat. If you switch to $16 \mathrm{~mm}$, the technology gives you hundreds of grains on that leaf.” (Mekas 2006)

Nevertheless, there is still a gravitational pull that makes the cinematic image nearly as immersive as a technologically unmediated experience such as a dream or wakeful witnessing. If contemporary viewers no longer naively assume the embodied reality of the film images, the "magic" of cinema remains strong in its capacity to pull induce a state of abandon akin to that of dreaming, or at least day-dreaming. At the same time, the viewers do not lose the memory of themselves or cinema's reference to "reality" even though sometimes, and especially in documentary and documentary-style films, may seem more convincing than life itself. While shimmering thought is experienced as the phenomenal content of body-mind, i.e. the medium of thought as a pre-reflective possibility, the film flicker is experienced as the phenomenal content of cinema which makes possible the formal enactment of the cinematic moving image. It is the pre-reflective textural plane on which reflection becomes possible. This brings up media not only 
as a means of communication or cognitive gathering but also, as Merleau-Ponty would have called it, "a sort of being" (Merleau-Ponty 1964, 43) that breathes itself into the world and breathes the world.

While much attention has been given to the form and formatting of cinema, little has been said about the cognitive ramifications of the film grain and the film-flicker - the technological mimickers of the shimmer of thought and thought snippeting that underscore the mediated enactment of the moving image. The rhythm of light in the dynamic composition of visuals has already been discussed by Steele and Logan in a 1964/65 paper on light variables. However, rhythm as a formal quality of light intensity belongs to the already consolidated identifiable layer of visual language. The flicker (the running of still frames at a certain speed, typically 24 minutes per second), on the other hand, is barely perceptible since it happens so quickly that is carried over by the visual memory (persistence of vision) into a perceived continuous movement. The flicker has constant rhythm produced from a projection speed with a specific (hypnotic) function - to bypass the speed of perception and align with the persistence of vision which makes possible the mnemonic retention and respectively, the apparent movement, of still frames. However, rhythm is not only textual, but also textural and this temporal tactility approximates it to the flicker. As such, the study of rhythm brings attention to a medium's potential for sensual unconscious conditioning of the viewer:

"The importance of light values and possible rhythms reach out from the screen like the flickering of a candle or the twirling disk which assist with the inducement of a hypnotic trance." (Steele and Logan 1964-65, 39)

The hypnotic trance that rhythm may induce is still a layer closer to the viewer's awareness compared to the fusion with subjective thought that the flicker can potentially produce. While the particle wavelengths of the vibrational band we assemble as physical reality are only 
commonly accessible through special scientific instrumentation, the shimmer of thought is directly detectable when in the state of rest and inner silence (including visual silence), i.e. it can be phenomenally experienced. However, it is completely undetectable when the attention is hijacked by the mental-sensory stimuli of wakeful life, and barely accessible in the high-speedattention state of dreaming. The film flicker provides a similar "carpeting" of the viewers' perception, securing not only the impression of "real" life the moving image provides, but also the impression of "real" thought. The reality of thought is thus actualized through cinema as a reality of perception.

But this realization is not limited to the shimmer and flicker as the vibrational carpeting of cinematic movement - it is even more apparent in cinematic movement itself. If we depart from Ramachandran's technical definition of language as "information-rich messages that are intended to convey meaning" (Ramachandran 1999 [1998], 231), a definition congruent with the hitherto explored relationship between language and meaning, and the wider sense of the meaning of language considered, we can talk about cinematic movement as the language of cinema. A language that organizes the formal elements of cinema within a certain syntax. Likewise, as unruly and amorphous as oneiric shapes are, as unstable they are in conveying meaning, they do move in ways that can be consolidated, if not into a syntax proper, certainly into a proto-syntax that reveals the way dream, as the door way of poetry, mobilizes meaning out of nonsense.

Dream images, unhindered by mass, move faster than the speed of massless light because they are also space-timeless. Dreams move at the speed of thought. While a photon would take some time to reach another galaxy, my thought reaches that same galaxy instantly upon thinking it. That thought which appears as the consolidated thought of a galaxy is neither here now (because here now I am writing at my computer, focused on this sentence) nor at the galaxy, and yet it's at both places as a here-here, not a here-there. This freedom of mobility of thoughts, 
travelling as they stay by simply taking the shape of the destination is akin to the shape-shifting freedom of mobility of dream images. The thought-form's agility comes at the expense of the misalignment with the energy field that would consolidate an image in the physical world, and would extend to the Heideggerian agglutination between thing and word. This is not to say that those images cannot be energetically aligned and that their phantom character is absolute or exclusive to the dream state, but that this is the default way in which dream images operate. Dream state allows us to observe how form unfolds on its own, unhindered by the spatio-temporal constraints of physicality. In wakefulness even a reverie or daydreaming can only be a mental fancy, invariably contradicted by the resistances of bodies - I may imagine myself taking off in flight at the end of my run, but while running, my body's incapacity to accelerate enough as to take flight reminds me of the lived laws of mass and gravity according to which this run is both possible and limited.

By allowing the free association and movement of the image (as experienced in thought and dream) as sensible phenomenon, cinema makes possible a hybrid experience of dream and wakefulness. The shimmer of light itself both grounds this experience of abstract form in sensible reality and invokes the primordial shimmer of thought as the feeling of reality, of energetic stability behind experiencing the body-world. In dream, control over speed and focus is greatly diminished and dreaming attention is typically dragged by the content of the dream, and away from its medium so cinema, with the shimmer and flicker grounds that content in the medium. This hybridization of the disembodied content and mediumatic grounding makes cinema uniquely immersive and life-dream-like. Cinema does become the "outside" sea of light where the subjective "inside" - the brain as the medium - floats unobstructed by physical laws and spaciotemporal limitations, while the viewers, thus disembrained, watch the unfolding of their dreams into reality. 
As already discussed, a particle that moves at the speed of light, a photon, arguably has no mass or form, yet it does have energy. Cinema is made of light - its images are animated not by energy of mass, but by energy of speed. While in physical reality light seems to only render things visible, in cinema it seems to make them be, intimating the crysopoeic potential of light. Constant massless speed begets an impossibility - variable massless speed. High speed formlessness makes visible low speed images, just as in physical reality, only in cinema those images are also massless. In a metaphor, in a dream, in a mathematical formula or in a straightforward document, the cinematic image is not only a phenomenon of movement, it is also a window to the movement of phenomena, a glance at the sea of reality as a projection on the shimmering walls of fantasy.

The affinity of media, cinema and thought in terms of vibrational experience is a barely examined (to my knowledge) aspect of the blurring of cognitive boundaries between film and physical reality. As we find ourselves increasingly immersed, indeed bombarded, by moving images, this blurring of boundaries is no longer just a curious illusion. It becomes a pressure that demands rigorous questioning if we want to avoid being dragged by it like a dreamer lost in the phantom world of his or her reality residue, where fragments of a hopelessly shattered herescientific there-metaphoric world float around blown by the wind from the Caspian sea.

\section{Dream}

"We are such stuff

As dreams are made on,

And our little life

Is rounded with a sleep." (William Shakespeare, The Tempest) 
"I think, therefore I am" - I dream, therefore you are. Cinema with its cognitive inversions and reversals intimates the possibility of a dream-like communion that resolves the initial separation in one interpersonal dream of humanity as a multiplicity of points of view that dream each other unto reality.

We wake up, open our eyes, and the day announces itself in the flicker of the light gliding over the pillow, in the movement of the leaves outside the window, in the passing of a cloud over the shimmering sun. Even if all the objects in our field of vision are immobile, they still breathe with the uncertainty of the day light, making the difference between a photograph and a still movie scene instantly discernible. In short, upon opening our eyes we find ourselves immersed in a world of moving images.

We go to bed, close our eyes and images begin rolling in the darkness. Blurry blotches of colour and truncated words float around - residual impressions of a day melting away. Slowly, they begin to take strange shapes that drag the focus from the environment outside to the environment within, making us fold upon ourselves like some Moebius tori. Soon, we find ourselves immersed into a world of moving images - the world of dream.

Cinema is often likened to dream but the similarity is not only metaphorical. Before the moving image was first captured and projected on screen, it could be experienced as a disembodied non-volitional phenomenon only in dream. While dream may be regarded primarily as a faculty of the mind (as an experience of the mind during the state of sleep), cinema is a wakeful, fully perceptual, experience. It could be said that dream is the most introverted experience:

"sleep is a condition... [where] no action takes place and even sensory perception is almost entirely shut off... While we sleep we are... helpless... But we are also free... from watching and mastering reality. We need not look at the outside world; we look at our inner world, are concerned exclusively with ourselves... we may be likened to a foetus or a corpse... to angels, who are not 
subject to the laws of "reality". In sleep the realm of necessity has given way to the realm of freedom..." (Fromm 1976, 26)

In other words, in dream we are conversing with our mind on its own terms. When we are fully awake, even if we drift into daydreaming, we are always concurrently experiencing physicality. As I am writing this, I do not pay attention to my fingers touching the keyboard, my feet touching the floor or the warmth of the heated room air around my neck, yet all those sensations run silently in the background of my attention to ground me in my environment and provide an empirical reference to my focus. All those sensations remind me constantly and beyond a shadow of a doubt that I cannot float in the air while writing this or that I cannot make a text appear on the computer screen by typing on the wall. In other words, those sensations represent sustained predicative experience that underpin an entire universal set of judgements that implicitly directs my active being in the world. However, in dream, I suspend judgement as (mental) alignment to physical reality, and can do all of the above, as long as my mind intends to. Unlike physical movement which happens by force(s), dream movement happens by intent. This intent is not the conscious intention of willing, neither the phenomenological intentionality of consciousness, but rather the dynamic momentum of all mental activity, regardless of its level of awareness. Unlike sensory phenomena which discipline the flow of mental activity, by resisting, holding and directing it, the dream image flows with the movement of thought. In fact, the dream image can hardly ever stop, demonstrating in a visionary (by means of visions) way the constant movement characteristic of mental activity. Stillness or immobility experienced perceptually is the resistances of physical reality to flow with thought; it is an effect of two different temporalities (the mental and the physical) colliding. The wooden table in front of me is visually static no matter how I move my eyes around it, change my body position, perspective or thought process. I know from experience that wood is slowly degenerating - an extremely slow motion 
process that I am incapable to detect as my body's perceptual speed is not aligned to that of the rotting wood. Therefore, I perceive the table as static at any given moment. By contrast, I can hardly sustain the mental image of the wood table if I close my eyes. Likewise, a dream table would shift and morph at the slightest blink of my attention. This facility at suspending predicative experience and respectively, judgement (in addition to the minimized perceptual functioning in sleep), of letting the dream imagery flow according to the allowances of mental mobility, gives us an opportunity to observe the behaviour of thought in optimal (subjective) isolation. (1)

The moving image of film, abstracted from its original embodiment, offers the possibility of a similar unhindered re-play, beyond empirical limitations - from reversible time to space unbound by gravitational laws, to action liberated from location or period. But, unlike dream, the moving image of film is presented to our wakeful senses, i.e. embedded empirically. What we have in film is a disembodied dream-like reality that is generated outside of us and is offered to our senses the way empirical reality is - it plays out as an externalized dream or rather, as though we are an internalized object to another's subjectivity, a homunculus in someone's dream-mind. This peculiar amalgamation of dream and sense reality has inspired many views and theories about the interchangeability of the two. Let's replay the neuroscientific interpretation of reality elucidated by Metzinger:

"The whole idea of potentially being directly in touch with reality is a sort of romantic folklore; we know the world only by using representations...” (Metzinger 2009, 9)

In other words, dream, cinema and empirical reality as distinct identifiable phenomena are qualities of the only space we "really" ever experience, "for our eyes only." 
If we extend this vision of reality to dream, it could be said that dream is the most direct experience we can undergo since, in dreaming, we are in touch with indexical representations (that represent themselves), with the only reality we can know - the interface between us and the potential information available for legible assembling through that interface.

A closer look at this model begs for precision - the conversion of data into a world happens in the brain which is itself a physical environment and, therefore a wavelength. Rather than belonging to two different spaces, wavelengths and sensory interpretations belong to two different layers of experience that coincide where there is no "in" and "out" relative to our brains. Thus, the oppositions of "in" and "out," matter and form, body and mind, then and now, belong to the same layer and are ways of describing that layer in dynamic terms, eloquently synthesized by Hegelian dialectics. Those oppositions cannot be accurately applied to the relationship between wavelengths and sensory/physical layer not only because of the implicit confusion such an application would insinuate but mostly for the absolutization of conceptual language it would endorse. Physicality, with its "syntax" of agglutinating patterns such as mass, shape, consistency, motion, is an interpretation so overwhelming and coherent that it feels like the interpreted. The only difference worth speaking of is the difference between the field of frequencies and the assemblage of a certain range of those frequencies into a legible (in this case physical) world. The legibility of this world is constitutive of and constituting both its physicality as interpretation and its interpretation as physicality. When we speak of matter and interpretation as two different concepts, we are only splitting in half the same dynamic phenomenon - the assembling of a world available to/through human experience. And we come to know this world as the world not by thinking about it, but by the feeling of being in it. That feeling is also the one that permeates the dreaming experience, uninterrupted, as the feeling of being in a (this time, dream) world. 
"But just as the sleeper - in this respect like the madman - sets out on the macrocosmic journey through his own body, and the noises and feelings of his insides, such as blood pressure, intestinal churn, heartbeat, and muscle sensation (which for the waking and salubrious individual converge in a steady surge of health) generate, in the extravagantly heightened inner awareness of the sleeper, illusion or dream imagery which translates and accounts for them, so likewise for the dreaming collective, which, through the arcades, communes with its own insides." (Benjamin 2002 [1988], 389)

If we replaced "the arcades" with "the world," this quote would be just as valid - the world as the collective body of the collective dream. Thus the claim on dreams that cinema exercises may not be as contradictory to its claim to reality (as language may insinuate). Cinema is both a mimesis of reality and a collective dream - a mimesis of the mind. On the other hand, the experienced difference between dream and wakeful reality may be greater than the ego tunnel suggests. Each of those moving image realms is governed by a different set of rules which, in the case of wakeful reality at least, are grounded in laws (such as the law of gravity) that outline the edges of the (physically) possible through the brushstrokes of the known and the unknown. (2) How much the rules that govern dream image mobility and the ways the latter responds to awareness are grounded in laws is an open question beyond the scope of this work. However, the rules of dream image mobility themselves are of direct interest to this investigation. (3)

When Descartes allegedly distilled the body from the mind, or vice versa, he didn't do much more than equate the body with mechanics and the mind with (post-linguistic) consciousness. This nurtured the idea that the mind is the avenue of freedom and the body is limited by mechanical bondage - an idea that has been dominating implicitly the Western world ever since. The free-floating Cartesian mind is where conceptual thought feels at home so, it has been given a special status as the seat of our identity and the promised way to freedom. Thus, the post-Cartesian mindset has been motivated to apply conceptual thought to physicality in the implicit hope of freeing ourselves from the latter's bondage. This is how science, the best 
environment for the unfolding of this process, began to determine our values as rooted in a worldview of problems (as limiting) and solutions (as liberating). And since Descartes' view of the body and matter was mechanistic, a conceptual view, the investment of the problem-solution effort was placed in technology as the physical product of science, and as (presumably) fully convertible with the body. But the exponential growth of technology inevitably began to dominate the mind-scape not only theoretically, in the computational views of the brain or the advancement of artificial intelligence, but also in bio-chemical and genetic applications. Thus, instead of easing ourselves out of the physical bondage, we layered our world with yet another layer of mechanical engagement that exercised its own peculiar way of bonding with and formatting the mind. But what if, instead of mediating the access to the mind through technologies, we applied physicality directly to the mind?

Dream would be the most clearly accessible laboratory of the mind. Applying our wakeful attention to dream, importing our somatic knowledge and memories into the oneiric would be to approach the exercise. Lucid dreaming is neither new nor esoteric, yet it is rather marginally explored both academically and colloquially. And yet it is the experience that may best intimate ways in which the interplay between matter and form, mind and body could align and sustain realities. The stability and coherence of dream images achieved by introducing wakeful attention into the mindscape feels as restricting as physicality. Of the two aspects of wakeful attention - mnemonic retention (which carries over principles) and distancing awareness mnemonic retention stabilizes dream images, giving them sensory resonance akin to the resonance of the physical world. In its turn, distancing awareness (becoming aware of the dream) allows for stopping the oneiric flow into a stable scene and introducing mnemonic retention. It also later allows for pulling out of the gravitational vortex of the stabilized dream world which begins to exercise its own restrictions. The challenges in flexing out of those restrictions reveals 
that the mind is subject to inertia and limitations no less than Descrates' body. The distancing way of awareness is therefore concerned with the bondage of the mind more than with that of the body. It is much more involved task to exercise dreaming awareness than to become aware of myself or my environment at any given moment of wakefulness. Distancing awareness comes much more readily to us in wakefulness where resistances and resonances of physical reality are already in stable relationships to the mind and thus facilitate awareness more readily. Awareness is a function of the original focus and a condition of symbolic and conceptual distancing in language and technology. Thus, it arises in interpretation - the freedom it affords is a freedom of interpretation. As such, it applies to physical and sensual reality as much as to the mental and the symbolic, and is not native to any of these. However, conceptual oppositions, as a manner of speaking, facilitate the shift of vantage points and thus the discipline of attention as awareness. This seems to be the gift of abstraction (that conceptual thinking upholds) - the discipline of attention as awareness in the shifting of the vantage point. The discipline of attention as awareness by intuiting the point-of-view is the gift of immanence, the gift of enmeshment and undifferentiated being. The mirror neurons of neuroscience, the empathy of phenomenology, the experience of art, are all ways empirical reality facilitates the opening and expansion of the (individual) point of view. The changing of the vantage point (concept) together with the extension of the point-of-view (experience) allow freedom not as freedom from, but as freedom to. And this freedom to be is the way of knowledge as a way to survey and experience altogether.

Cinema - a techno-scientific way to induce oneiric experiences on wakeful terms - is therefore a syncretic conduit of both abstraction and experience. The cinematic moving image crowns the techno-scientific effort to export the body-mind into technology. It has succeeded so thoroughly that the cinematic experience mimics both the physical and the mental in a sensibleoneiric entanglement - an ultimate experience. Moreover, in this entanglement, it expands both 
the physical and the oneiric in ways unique to the camera eye-brain. In slowing motion to reveal detail inaccessible to the naked eye or closing up to break the barriers of distance, the camera expands sensory perceptions bringing to consciousness what Benjamin called "the optical unconscious" (Benjamin 1936) Through the camera-eye we can witness the bloom of a flower or the flight of a bullet alike, we can see a beating heart inside the body or a view on Mars. Conversely, in jumping through time and space, even the most basic cinematic editing, e.g. shot/reverse shot or establishing shot/close up, makes the oneiric available to the analytic mind. Only in dream we can move instantly from one room to another without having to walk the distance or shift our identity while retaining the same point of view - montage makes those experiences available in wakefulness. In short, cinema grounds the conceptual into presence, relieves apparent space, time and the physical from their usual constraints, and brings the oneiric to conceptual attention. It crosses all cognitive boundaries, not only giving flesh to the thinkable but making explicit our ways of assembling realities, affording yet another interpretative degree of distancing that unfolds the oneiric project into wakefulness. This awareness of the structure of private experience that cinema makes collectively available personalizes it and makes the viewer both vulnerable to absorbing its format as his or her own experience, and at the same time to feel competent, in control, as Benjamin observed:

"It is inherent in the technique of the film... that everybody who witnesses its accomplishments is somewhat of an expert.” (Benjamin 1936)

However, cinema is only a crude emulation of thought and the oneiric. Its interpretational loyalties remain with industrial technology to which it originally belongs. As such, cinema has its own way, which is neither the way of dream, nor the way of sense reality. It is the way of the machine, the language of the machine. But where is that machine? How does it dwell with the human? 


\section{The Cinematic Machine}

The machine is the Homo Sapience, the distinguishing species, of industrial technology. It is the moment where technology begins to forge its independence from the human and starts performing on its own. The machine is the beginning of the competition and confrontation of technology with the body. But this confrontation is also a confrontation with technology itself because the original beauty of technology is that it forges a relationship of care between the artisan and the tool. Heidegger has elaborated this point at length, but we don't need to go into his theory to illuminate this. Culture means to care, i.e. to have a relationship with the environment toward betterment. Care is a pause before action that opens up space for dialogue. In this dialogue, as short as it may be, the primate asks the environment and the latter gives - the primate reaches out for a grass blade to poke in the ants' hole, both for protection and aggression (selfcare), revealing and extracting. Care is a relationship of knowledge. The machine declares its independence and has no interest in the human-in-dialogue. It has mechanized the relationship and minimized human questioning, and the cognitive cooperation between technology and human. The machine does not need the human even for the generation of knowledge - it can generate its own informational algorithms and present them as revelation. This hostility of the machine toward the human is precisely distilled in the aesthetic programme of Dziga Vertov for film to remain true to its technological imperative as an art of mechanical movement:

"In an art of movement we have no reason to devote our particular attention to contemporary man. [...]

For his inability to control his movements, WE temporarily exclude man as a subject for film.

Our path leads through the poetry of machines, from the bungling citizen to the perfect electric man." (Michelson 1984, 7-8) 
And this art is not a mere recreational item aimed at entertaining and distracting, it is a powerful tool of shaping the human form itself, of cultivating a qualitatively new human. This new human is born of the loving intertwining of man and machine, a new kind of cross-breed, a centaur, a cyborg:]

"In revealing the machine's soul, in causing the worker to love his workbench, the peasant his tractor, the engineer his engine -

we introduce creative joy into all mechanical labour, we bring people into closer kinship with machines, we foster new people.

The new man, free of unwieldiness and clumsiness, will have the light, precise movements of machines, and he will be the gratifying subject of our films." (Vertov 1919, 8)

This would be the achievement of a cinema true to its essence, purged of literary, musical and theatrical influences and tapping right into its mechanical heart:

"Everyone who cares for his art seeks the essence of his own technique. movement.(...)

Cinema's unstrung nerves need a rigorous system of precise

Radical necessity, precision and speed are the three components of movement worth filming and screening.

The geometrical extract of movement through an exciting succession of images is what's required of montage.

Kinochestvo is the art of organizing the necessary movements of objects in space as rhythmical artistic whole, in harmony with the properties of the material and the internal rhythm of each object." (Vertov 1919, 7-8)

However, the cognitive reformatting of the human according to the mechanical movement of cinema is not an evolutionary trajectory, as Vertov enthused, but a trajectory of relinquishing power. Why? The precision of mechanical movement is possible because of its simple linearity. Linearity, simple or not, is not native to human thought, as its most reductive characteristics - dream, the shimmer and the snippet - reveal. Yet film viewing is a cognitive experience - cinema does not train the movement of the human body, it affects the movement of the human mind. Thus, a mimetic experience of externalized thought processes cannot produce the uber worker Vertov dreamed about. However, it could produce a complacent worker, not 
necessarily more physically precise than before, but certainly more aligned with the simplicity of "machine thought." Yet, even this complacent worker is not the result of Vertov's cinema. By contrast Vertov's cinema, by lying bare the mechanistic essence of the medium in building a language true to its principles of speed and geometry, confronts the viewer with the alien nature of cinema. The poetry of mechanical movement can thus invite contemplation, a sort of machinegazing, which could accelerate perceptual speed and fine-tune geometric pattern recognition, but this would hardly translate in the centaurial fusion of the peasant and his tractor. Rather, Vertov's art, capitalizing on the perceptual extensions cinema introduces, allows the viewer to absorb the cognitive edge of the machine while retaining a critical distance from its workings. However, the machine is a human product and, as such, it distills principles that already exist in the human realm. The regularity of the heart's rhythm, the speed of thought, the discontinuity of thought snippets are all prototypes for the building blocks of Vertov's mechanistic cinema. That is why they can be swiftly naturalized and internalized when other art forms are invited into the cinematic space. Then the mechanical language of cinema may be obfuscated and turn subliminal, unconscious, in its formatting power. From here the idea of a thinking machine becomes less and less fantastical, not because the machine has indeed proven human-like but because it trains the human to focus on conceptual thinking and overlook those aspects of the thought process that do not translate in machine-speak. And which comprise the domain of thought.

Last night I dreamt that I was looking at what was both my toes and my fingers of what could only be articulated as hand-foot. However, in my dream, this particular anatomical part was very clearly what it was and I was observing its details with great care, and without a shadow of confusion. As I am trying to articulate this dream in writing now, suddenly I am at a loss of how to unpack it. The moment I try to pin words to the visual memory, it turns into something entirely different, and both the certainty and the fullness of the feeling of the memory is lost. Instead I am 
left with a grotesque term hand-foot while neither "hand" nor "foot" were components of the dream.

Even more explicitly, yesterday, as I was walking down the street, I had a flashing thought. I automatically started to try and share it with my companion when I was stopped in my tracks. The thought, although at most a second long, was full of so much information, spanning to my personal history, through the history of the world. It was contextualized by two different cultures and referred to genetics, aesthetics and social mores all at once. If I were to unravel this knot, it would take me a long boring hour that would completely defy any reason why. Instead, I opted for a short excerpt, an incident that seemed to summarize crudely some of the points touched by my thought.

In both cases, time-space had collapsed into a multi-layered instant of compressed information that made perfect sense to me yet defied direct linguistic unpacking. I had to turn to language for something that most closely conveyed my thought experience, without necessarily even describing it precisely. This collapsed multi-layered, polysemic space-time, or rather nonspace-time, is not available to technology - a product of conceptual thought and a native of space-time. By losing track of this, we lose track of thought itself, because conceptual thinking without thought itself, is only a projection of mechanics. Thus the cinematic camera can capture and arrange in movement, animate that is, any image that the filmmaker can think of and make a drawing, a collage, a print, a painting, a sculpture, and anything that could be rendered in another medium or directly on the celluloid film - but it cannot capture the thought itself.

Vertov's cinema of speed aligns with thought as described by speed rather than duration (the domain of sense experience), hence its aesthetics has a specific cognitive potential that can amalgamate the machine movement and human thought in ways that may undermine the natural flow of the latter and subject it to a machinist cognitive imperative. 
"The camera developed in response to a crisis of sensation, as a cognitive tool that served as a prosthetic for vision. Yet the modernists recognized that the camera's eye - including that of the movie camera - posed significant aesthetic difficulties. For if the camera was invented as a cognitive tool for revealing reality rather than for transforming it into an autonomous form, then it is difficult to see how the photographic (or cinematographic) image can be of much use in carrying out the artist's task - that is (according to the time's reigning aesthetic principle), in constructing artistic forms. Indeed, it is even difficult to see how a camera image could possibly provoke an aesthetically valuable experience." (Elder 2010, 4)

That is, if the term "cognitive" were read in the narrow sense as the investigative scientific imperative built-in the invention of the camera, the the clash between the industrial technology of cinema and the poetic energy of arts became dramatic. This conflict was addressed by a 1920 movement called Absolute Film, a group of German artists who used film as an extension of the traditional arts in motion:

"The Absolute Cinema answered these objections about the character of a camera image. The makers of Absolute film proposed to avoid using the camera to reproduce the appearance of reality, and even to avoid reproducing movement. Instead, they would create films from concrete forms, forms they would create by hand (using cut paper or cardboard, paints, and dyes) - forms that would lack any representational import; they would use the camera not to reproduce movement but to create a pure, artificial dynamic." (Elder 2010,4)

In exploring the capacity of cinematic movement, Absolute Film goes beyond Vertov's documentary camera-eye - not only it does not need durational continuity, it can do away with photography altogether. Direct to celluloid techniques could do away with the camera altogether thus making the non-documentary, non-reality-bound, thought-in-cinema-making more explicit.

With the advent of cybernetic media technologies, the camera became entirely unnecessary whenever most of the animation techniques, not just the direct-to-celluloid ones, were concerned. Paintings, drawings, 3-d models could all be scanned in, treated or generated directly in the computer. An entire film can be made without a single camera shot, thus both returning cinema in the hands of the artist, and pushing it further away, into a machine that is 
much more linguistically elaborate than the film camera, and therefore much more opaque and distancing in the freedoms it affords. This makes the non-alienating distancing that poetic language in art affords both more necessary and less obvious in a new mediumatic expanse that obfuscates poetry by promoting spectacular discontinuity.

Conversely, the continuous camera of Jonas Mekas, flowing in the now, uninterrupted even by its own flaws, brings us the other end of cinema - cinema as a document of perceptual reality. The camera of Jonas Mekas is no longer a camera-eye, it is an eye-camera - a cyclopic extension of Mekas' own eye, a true cyborg. Only it is the machine that becomes humanized, not the human eye mechanized. Indeed, the camera becomes infected with the human imperfections Vertov was so weary of.

“We need less perfect but more free films.” (Mekas 1972, 1)

It is a poetry of perceiving in the now, of witnessing the beauty of simple everyday experiences brought to us with all the stutters, stumbles and glares of the eye-camera. It is a spontaneous personal act:

"Because I film only very personal moments, nothing preplanned, staged or written, it has to be real and spontaneous." (Mekas 2012)

The impressionistic patience of Mekas' camera, its fascination with a sea tide or a bowl of fruit, challenges Vertov's devotion to speed not defiantly, but dialogically. It makes explicit the other end of cinema - cinema as document of the sense reality, as memory, as witnessing companion. It is a cinema married to photography, emerging from photography. But, instead of basking in the possibilities of technology, it reveals its limitations, and by extension it reminds us of the limitations of natural optics, without obfuscating the difference between the two. The eyecamera brings to attention the structure of the optical experience in motion while, by allowing the technical glitches of the machine's optics within the shot, retains a poetic distance between the 
viewer and the screen. However, as with Vertov's cinema, the poetic eye-camera of Jonas Mekas, if, for instance, edited within a dominating literary movement, a story, can easily be naturalized as the way of perception and thus obfuscate the difference between the viewer and the cinematic machine.

Thus, on one end of cinema stands the mechanical camera-eye of Vertov, grounded in technology, industry, speed and precision. On the other end, the organic eye-camera contaminates technology with the slow patient gaze of nature, the body, the quirks of chance. In his theory of montage, Eisenstein offers a synthesis of those two ends of cinema in a dialectical vision of art that projects though every layer of film language. He identifies Nature as the limit of organic form and Industry as the limit of the rational, the collision of which produces Dynamism:

"Not only in the sense of a space-time continuum, but also in the field of absolute thinking. I also regard the inception of new concepts and viewpoints in the conflict between customary conception and particular representation as dynamic - as a dynamization of the inertia of perception - as a dynamization of the "traditional view" into a new one." (Eisentein 1977 [1949], 46)

This principle is expanded into a dialectical conflict-resolution, tightening the relationship between elements within different levels of semantic layering of film language in a way that a conflict resolution on one level becomes the conflict element of the next, in a precise aesthetic rendition of Hegelian-Marxist dialectics:

"Conflict within a thesis (an abstract idea) - formulates itself in the dialectics of the sub-title - forms itself spatially in the conflict within the shot and explodes with increasing intensity in montage conflict among the separate shots. This is fully analogous to human, psychological expression. This is a conflict of motives... This gives us the basis for an entirely new understanding of the problem of film form. We can list, as examples of types of conflicts within the form - characteristic for the conflict within the shot, as well as for the conflict between colliding shots, or, montage." (Eisenstin 1977 [1949], 54-55)

In the dynamic tension between opposing elements, Eisenstein sees the resolution of the question of cinema. This resolution lies into cinema as art (a term that both Vertov and Mekas shy 
away from) optimizing its medium-language, and submitting all possible "contamination" from other art forms to its semantic imperative. True to the nature of cinema as an industrial technology, this resolution is conceptual, yet the concept itself is based on the incorporation of its opposite. Eisensteinian montage (theoretically) allows for the incorporation of camera-eye, eyecamera and no camera techniques in a balance that guarantees the distancing pause poetry affords.

Those film-making approaches not only represent imperative bands of the film-art manifold, but also the continuous effort of artists and poets involved with the medium to tame its alienating edge, on one hand, and to keep a distance from its possibility of exercising industrial hypnosis, on the other. Film as art (in the narrow sense of distancing that those techniques afford), cannot become a hypnotic event, a material addiction, because the communion that it can offer is a highly energetic state of singularity. An art work, be it Vertov's machine-inspired fast cuts, Mekas' flawed reveries or Eisenstein's intense collisions, is a work of high semantic condensation - it is brimming with explosive energy that pushes its own boundaries and, as such, threatens and promises to blast us in the unknown. This is why an encounter with an art work is a confrontation that can either result in repulsion and avoidance or with an explosive unrepeatable communion. Such a high-voltage encounter calls for awareness and energetic mobilization - a state too intense to induce addiction and hypnotic dependence (the two sides of technological alienation). An art film, even if viewed repeatedly, is therefore always revealing new sights, new layers of its manifold. Its attraction is the attraction of the unknown, peering through the cracks of language tensions, be it the tensions between natural perception and the rapid camera-eye, rushing thoughts and the patient eye-camera or the dramatic tensions between formal layers, it keeps the viewer engaged with the promise of the unknown. The viewer is engaged in an act of knowledge. With every subsequent viewing, the cracks open up more and new layers emerge. It is never the same film. As such, it always keeps the viewer either alert or disinterested, but never subjugated. It 
optimizes the tension distancing of language-awareness so that the viewer cannot be bedazzled even by the most spectacular effect. In other words, even the most spectacular art film cannot be a spectacle.

Industrial technology needs spectacle - the simple explosion of fireworks, repeated over and over. The firework patterns are no issue - regular or irregular, random or programmed, it all takes place on a single layer and taps into the mechanical repeatability of the experience. The fireworks quench the human thirst for thrill, novelty, predictability and sensory excitement without calling forth awareness. It is always at the same distance - accessible yet alien. The fireworks seem poetic - they surprise us in the night sky as non-linear explosions of sensory stimuli - they come and go like magic, they change rhythm of movement and colour, they thrill and dazzle like incredibly close falling stars making us feel at the edge of open space. And yet, they confront none, they reveal nothing - they thrill, dazzle and mesmerize - they are a perfect simulacrum of poetic experience.

Industrial technology, sustained by the ideal of infinite repetition, gravitates toward forms of cultural production that can foster addiction - the continuous chain consumption of a simple repeatable formula, in slight humanistic variation. For this, the form must be overwhelming, stimulating and satisfying the mind-body without allowing it entry into the process. It has to mimic the art process without any effort on the part of the viewer. Cinema is the perfect vehicle for such an arrangement, but even more complete in its simulating capacities is, the pregnant with interactive possibilities, cyber-cinema. Interactivity adds a physical engagement in the simulation process that is impossible in the traditional cinema setting. Thus it can provide the perfect mirror surface for human cognitive processes. Always at a precise distance it thus allows humans to use it as a mirror, a constant moving mirror reflecting humans, reflecting technology. Thus cinematic production that doesn't account for the non-alienating distances afforded by art cinema remains 
open to being possessed by the spirit of technology. This spirit, as far as cultural forms are

concerned, is a spirit of spectacular alienation, an alienation that would drive humanity to

"experience its own destruction as aesthetic pleasure of the first order." (Benjamin 1936)

\section{NOTES TO CHAPTER 3:}

1. Here I would like to clarify that, when discussing the dreamed image, my questioning concerns strictly the characteristics and behaviours of the moving image and not its possible psycho-neurological wiring, neither any possible causal relationship to empirical reality. When I say that the dreamed moving image does not obey empirical laws, I mean that in dream the moving image of a car, for instance, may or may not crash into a wall. It can also melt into the wall, pass through it or fly over, among other imaginable possibilities. When I say that in dream I suspend alignment to physical reality, I do not mean severing ties with my body, which always remains the physical environment that carries forth the dreamed experience. I am simply focused on the experience itself. By extension, I do not mean that the oneiric imagery is not rooted in empirical reality, such as for instance Bergson's suggestion (Bergson 1958, 30) that the dream experience is an associational afterthought of physical stimuli. This may or may not be the case of dream triggering, but it is beyond the scope of my focus on the syntax and movement of dream imagery as directly experienced by the dreamer.

2. If we take, for instance, the law of gravity, on one side it allows us to manipulate the known and construct air planes and space ships, and on the other, it restricts us by guaranteeing that, if one of us jumps form the Empire States Building, they would most certainly die, i.e. face the unknown.

3. My use of the term "rule" here is provisional - I am referring neither to institutionalized principles (of behaviour) nor to conceptualized "laws" (as conditionally borrowed here from Physics) but, rather to (cohering as) patterns that are dynamically related in a network articulated as such through the subjective grasp whereby that network emerges as a coherent world, not unlike Bohm's holomovement. This is why it could be said that language, as a dynamic network of patterns, is already present in the prelinguistic universe. Indeed the pre-linguistic universe, as far as it is assembled through perceptual articulation, is a language, a non-symbolic language that thickly overlaps and conceals the bands of the vibrational field it refers to. This language-body/mind-universe is opaque and heavy, it is like a hidden door masked as a stone wall, protecting us from the winds of the unknown and perhaps more importantly, the hurricanes of the unknowable. While the unknowable cannot possibly be imagined and therefore proposed (how can I ever know there is an unknowable?), it is nonetheless thinkable and therefore supposable. The unknowable is what emerges at the radical edge of reason and therefore, I would like to keep it in view, if only just poetically, as a humbling reminder of the ultimate limitations of this discussion and all that it may wittingly or unwittingly refer to.

\section{Chapter 4. Myth, Prophecy and Technology}


As the lights of the movie theatre dim out, Plato's allegory of the cave is hardly an original detour of my mind. It is the first documented fantasy about a movie theatre. And the historical beginning of the paradox behind my questioning. A cave is an opening, a shelter, a mystery, a trap, but a cave is also a pupil - a pupil that doesn't dilate or contract - it is frozen, carved in stone, staring blindly at the world. Caves are the wombs of the Earth, but the motherly solace and in-sight they offer is laced with the dangers of secret knowledge once guarded by dragons.

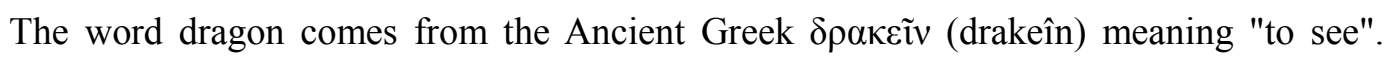
With their unobstructed vision, ancient European dragons would spot the intruder to their guarded domains from far away. Their ocular prowess was matched by their ability to take off in flight from the depths of the Earth into the high skies (a veritable chthonic creature, a serpent, the dragon was also endowed with the gift of the heavens, a pair of wings). In other words, an ancient cave was not only a cavity into the gutters of the earth but also an opening into the skies above. Like a primordial camera obscura, the opening of the cave was not only the pupil of the cave into the outside world but also the opening of the outside world into the cave - an embodiment of the ancient solar-chthonic dichotomies. This makes the cave all the more appropriate as the setting for Plato's ocular allegory of knowledge and reality. However, Plato made little use of the optical potentials of the ancient cave. Indeed, he made little use of his own allegory. It is not until the advent of cinema and the event of the movie theatre that this allegory could unfold and transcend the domain of the imaginary that Plato promoted.

In his allegory of the cave Plato ties up its inhabitants and immobilizes them so as they can only perceive echoing sounds and shadows projected on the wall before them. Since they are immobilized, they have no way of establishing the source of those audio-visual reflections which are therefore presumed to be self-contained and thus representative of reality. Only a man 
liberated from this position can wonder around, find out the source of the shadows cast by actors moving behind the viewers, and walk out of the cave into the sun light. Although the enlightened man makes ample use of his sense of sight, the point Plato seems to make is that the senses can deceive us, that truth lies beyond the sensual realm and that true knowledge can only be achieved through pure reason unruffled by the deceptions, limitations and concerns of the senses. However, Plato's man of knowledge does not achieve his enlightened state by closing his eyes and abandoning himself to the vacuum of a senseless world - he achieves it by untying his body, the seat of the senses, thereby liberating it into movement and full sensory experience. In short, his cognitive enterprise is only made possible by liberating the senses and engaging them fully, rather than suppressing or negating them. Like the eye of the dragon, he emerges from the depths of the Earth to face the sun and the stars.

During Plato's times a cave was the mysterious dwelling of the oracle whose body Apollo would possess to mediate his prophesies. According to legend, Apollo had slain the dragon Python whose body turned into an underground spring emitting vapours through the cave's opening. The site dedicated to this event was located at Delphi, a name rooted in the ancient Greek word for "womb" - delphys ( $\delta \varepsilon \lambda \varphi v ́ \varsigma)$. Apollo, the sun god of light and music, manifested himself at the opening to the womb of the earth, and into the body of the sibyl intoxicated by the chthonic fumes of an underground spring. The dreams of the Earth's womb would fuse with Apollo's light and sound to bring forth visions beyond time-space. In winter, Apollo would retire and Dionysus would take over his dwelling. Just like in ancient Egypt, where a staircase would link directly the tomb of the dead, the world of Osiris, with the star-covered body of Nut, the goddess of the sky, Earth and Light were intimately intertwined at the time Plato wrote his allegory. Like Plato's man of knowledge, the oracle of Delphi linked the two worlds to bring forth knowledge. The gulf between the light of the skies and the darkness of the Earth's belly, between 
the Apollonian and Dionysian energies, was not nearly as clean-cut and deep as Nietzsche, still caught in the conceptual necessity for oppositions, tried to rationalize poetically. They were entangled into a moebius fabric that made distance only a matter of optics - where the immanence of the Earth was transcended, the immanence of the sky began - the body of the Greek Earth was covered with the body of the Egyptian sky, the Greek chthonic female and solar male, seen from the angle of the Nile delta transgendered into the male Earth and the female sky. The dark depths of the Earth dreamt forth the starred depths of the sky and vice versa. The access to the distant stars was through the womb of the earth when Plato wrote his allegory, and these were the cosmic mysteries he was culturally initiated in so, it stands to scrutiny that this more subtle view of knowledge was not beyond his consciousness when he crafted his story.

The wandering of the ancient man of knowledge in the depths of the Earth to reach the light of the skies shows how transcendence and immanence are the the two bands of the moebius strip of knowledge. The depth of the body catapults us to the stars, the womb of the Earth is the seat of knowledge and the conceptual tools are the strings on which the earth sends us as scouts to roam among the stars. Dreaming, thought, the play of shadows on the cave's walls, dwells in the womb, in the cave, in the darkest, most secret part of the body. The theatrical cinema recreates this dream-light in the womb as the cosmic flight from the womb. Immobilized like Plato's captives, we watch the dream projections of the cinema made of pure light as though we were in fact looking through the opening of the cave into a distant time-space brought to us not by the fumes of the earth and the light of the sun, but by the fumes of machines and the light of electricity. This capacity of cinema to recreate the most intimate and ancient archetype of accessing knowledge, of communion with the world, makes cinema both deceptive and conducive of cognitive receptivity. 
Further in the same chapter VII of The Republic Plato argues for the supremacy of mathematics as a purely rational art unfettered by sensory considerations. And although he admits that mathematicians are preoccupied with the practical applications of this art, he ignores its empirical entanglement. Since Plato, we have been growing increasingly accustomed to think of rationality as divorced from practice. The most dominant questionings of this dichotomy tend to play with a prioritizing relationship between the two as though they were crooked mirrors of each other. To behold the sight of the sun, the liberated cave man has to adjust his sense of vision, has to push his sensory capacities to their full potential. Likewise, to acquire the art of mathematics, the child has to go through enough experience of discreet instances in the world as to be able to synthesize rules and repetitions. Before I stand accused of naive realism, I would like to emphasize that the preceding statement does not exhaust my position on mathematical cognition but only initiates a line of argument away from a dichotomy that has needlessly encumbered human thought for centuries. In doing so, I am not seeking to establish a rational or empirical monism as has been the predominant theoretical habit to date. The reason I advance mathematics as empirically based is not because of some strongly held belief in the priority of experience over thought - the empirical-rational duality is only a dialectic nominal classification within the same cognitive paradigm, referring to two aspects of an integrity - that of physical reality as we corroborate it. This is not an advocacy of physical reality as the only possible reality, but merely positioning physical reality as the departing point of collective reference or reality-station, so to speak, that makes the present discourse both possible and intelligible. Mathematics as a rational discipline is, narrowly speaking, a language of logic, is a linear way to knowledge - to achieve a logical conclusion one has to follow a demonstrable sequential procedure. In terms of human experience, the only place where such linearity holds a claim to validity (or reality as the discursive case may be) is physical reality in so far as a physically consolidated object cannot be 
interacted with, bypassed or overcome in non-linear manner - it obeys and imposes a linear behavioural protocol that enables and limits its extending in the world. A solid physical object cannot pass through another solid physical object without damaging its integrity. The sequential behaviour of physical bodies, from the observable celestial bodies in the night sky to the observed terrestrial bodies in our daily lives all follow uninterrupted trajectories, condition a sequential mode of cognition, of getting to know the world that is, in its pure form, rationality. Rationality is an optimization of the linear principles of action by which physical bodies behave in three dimensional space and linear time as perceived/conceived within the given human sensorialconceptual apparatus we operate in. How does a material body move from point A to point B is the original question of rationality - a question answered by mechanics. What is the optimal uninterrupted route from point $A$ to point $B$ is the original question of rationality - a question answered by geometry and visualized by the straight line. While energy describes the basic condition of animation, the straight line describes the fastest route to ending an active energetic state. Both energy and the straight line have received little publicity outside physics and geometry, yet they are the building blocks of the edifice of rationality - the main constructive preoccupation of post-Platonic (Western) thought.

Vertov foregrounds speed (energy) and geometry (straight line) as the essential elements of a cinema true to its technology. Those are not only the principles of building and functioning of the cinematic machines (camera and projector), they are also the principles cinematic language is built on - the fast-paced projection of still frames at speed higher than the capacity of the human eye to detect discontinuity is coupled with the linear geometry of the celluloid film itself which thus becomes subject to linear time. Not only does a film projection run straightforward, one way, it is also a fixed uninterrupted length. Unlike a book, which can be read at different speeds, a film is only viewed at its own pace, on its own temporal terms (in order to have its intended effect). 
With the advent of interactivity, from the primitive remote control to the advanced branching digital cinema forms, the primacy of the machine is pushed back, obfuscated by the foregrounding of (code) language which now stands between the machine and its final linguistic product. Cinema is now encoded in a completely different type of language, a poetically dissimilar inter-layering that both allows for flexing off the spacio-temporal grip of speed and line, but at the same time pushes the material base of the language further away from user's/maker's understanding. The line and speed are translated into 0 and 1 - the hitherto most convincing way of demonstrating the generative powers of mathematics and the binary principle, a Platonic triumph.

But the geometry of physical reality is predominantly fractal and does not lend itself easily to the straight line. The only place where a straight line can be observed without human intervention is in the distant horizon of fields and open water. By transplanting the straight line from the horizon to the proximity of here and now, rationality reverses a relationship that kept the knowable unknown at bay. In other words, instead of climbing out of Plato's cave, the Philosopher observed the sun as it rolled into the field of vision of the cave's mouth, took its disk shape into his mind and drew it on the cave's walls, inaugurating the rational circle. Or at least this is what Plato's most vocal interpreters did with his allegory.

In the age of genetics, it is not hard to correlate the unfolding of an organic body with a preliminary DNA code that frames the body's eventual manifest parameters. In the world of neuroscience, it is not farfetched to think of reality as given by our brain (image) maps that allow and restrict us to assemble the world precisely the way we do. After all, our perception can be viewed as the interpretative construction of our perceptual apparatus in terms of the stimuli that it can detect. The grass my hand is lying on is spiky, green and supple to my eye and touch, but it may also be a myriad of other qualities that are simply inaccessible to my particular perceptual 
apparatus. Or, it may be something entirely different if I shift my attention - a soft and cool ground to step on or a conglomerate of distinct living organisms, each unique in its class of grassness, each one surrounded by unique circumstances. I close my eyes and touch the grass, and I can feel a fabric of distinct sensations on the palms of my hand. They are hard to describe as they become a part of my hand through the amalgamation of spikiness, coolness and softness, of individual shapes pressing against my palm within a particular (inarticulable) register of resistance and fusion. This feeling becomes slowly indistinguishable from me and, in its unity, loses all conceptual ground. Indeed, it is not beyond my human capacity to venture into breaking it down in rational thought and mapping it out in written language, but the time-energy demands on such an exercise would make it as futile as killing a tree by plucking out its leaves one by one. The experience of sense reality is just as absurd to unpack in linear language as thought snippets or thought compounds. Metonymy, my entire body poured into a part of itself, into my hand touching the grass, and synthesis, my conscious distancing and linguistic ability to organize the myriad of sensations into a single experience of touching grass demonstrate how sense reality is organized by thought, just as thought is disciplined by sense reality. Conversely, as discussed earlier, rationality comes into language from manifest/sense reality, from the Aristotelian "habits of nature" so to speak, yet manifest reality itself could be viewed as blooming from language, if by language as a system of organizing information we can also mean organizational principles of matter such as the DNA code. But just as poetic language creeps up on us through dream to meet rational language, in its turn creeping up on us from empirical reality, so does poetic energy itself burst up through empirical reality to meet the rational distancing that comes to us through the discipline of consciousness. The Delphian oracle needs both the energy of the chthonic fumes rising up from the belly of the Earth and the Apollonian spirit to descend upon her, just as the 
artist, the one that unites all conflicts through beauty, needs both the duende and the language to channel it:

"So, then, the duende is a force not a labour, a struggle not a thought. I heard an old maestro of the guitar say: 'The duende is not in the throat: the duende surges up, inside, from the soles of the feet.' Meaning, it's not a question of skill, but of a style that's truly alive: meaning, it's in the veins: meaning, it's of the most ancient culture of immediate creation.

This "mysterious force..." is... the spirit of the earth, the same duende that scorched Nietzsche's heart as he searched for its outer form... without finding it, and without seeing that the duende he pursued had leapt from the Greek mysteries to the dancers of Cadiz and the headless Dionysiac scream of Silverio's siguiriya." (Lorca 1933)

"This mysterious force", "the spirit of the earth", that is "of the most ancient culture of immediate creation" is beyond language, it is the creative source that perhaps gives rise to language itself but which, nonetheless needs language to retrieve, to open up the crack for its insurgence. It is language that needs this force in order to come to life, it is Apollo who needs the intoxicated body of the oracle in order to bring forth his own words. It is the force that creates dreaming as its poetic gateway and then, through the discipline of language that poetry brings us back to the silence, back to the creative, back to the mystery of death:

"The duende... won't appear if he can't see the possibility of death, if he doesn't know he can haunt death's house...

With idea, sound, gesture, the duende delights in struggling freely with the creator on the edge of the pit. Angel and Muse flee, with violin and compasses, and the duende wounds, and in trying to heal that wound that never heals, lies the strangeness, the inventiveness of a man's work." (Lorca 1933)

But how can this creative energy enter disembodied cinema? Where is the body of cinema and how can it be wounded or opened up to death? How can it become "death's house" when it can never die, forever re-playable by forever reproducible machines, never original, always a copy? Filmmakers from Len Lay's 1935 direct to film promotion of the British Post Office to Bill Morrison's Decasia (2002) have been trying to ground cinema in its material body, 
but it keeps leaking through the indifference of reproducibility. This is not the judgemental indifference of attitude, but the qualitative indifference of projection. The life of film is in its projectability - it makes its material body irrelevant because the copy is as good as the original as long as it delivers an identical projection.

Decasia, the 2002 film about the mortality of the film stock as the cinematic body illuminates the illusion of stability that the cinematic reproduction perpetuates. It brings to the visual fore that cinematic layer that contains the possibility for a unique presence of the cinematic work, the original bearing the marks of death's touch. Yet, like a shot of a dying body, the decaying film of Decasia is frozen in time by the possibility of endless reproduction. The film work does not reside in the original copy, it resides in the active projection. The body is irrelevant in film as long as its image is captured and recorded. Like a reenactment of life after death, a cinematic work lives long after all the bodies, materials, actor, places, connected to its unique moment of making are forever gone. Cinema is life after death.

Hours before his execution Socrates makes a leap of faith, or reason as it may be, that would haunt humanity for millennia to come. He argues that the soul is the imperishable seat of knowledge that abides in death just as the body abides in life. With a string of allegorically connected dualities Socrates builds an argument that ties death to life in a regenerative causal cycle:

"And there are many other processes, such as division and composition, cooling and heating, which equally involve a passage into and out of one another. And this holds of all opposites, even though not always expressed in words-they are generated out of one another, and there is a passing or process from one to the other of them?" (Plato (2) 2009 [360 BC]).

It is not arbitrary that the examples above are taken from mathematics and mechanics, before he continues with a focus on experience: 
"Well, and is there not an opposite of life, as sleep is the opposite of waking? (...) And these, then, are generated, if they are opposites, the one from the other, and have there their two intermediate processes also? (...) Then suppose that you analyze life and death to me in the same manner. Is not death opposed to life?" (Plato, Ibid.)

A poetic leap is enmeshed with rational conclusions - biological and lived processes swapped with mechanical and conceptual ones. If the argument is kept within one cognitive domain, the duality it tries to establish would hold sway - if considering the behaviour of matter as we know or apprehend it, dead matter seems indeed eventually regenerated in live bodies (e.g. the decay of organic matter into the soil to feed back into plants) and thus it could be said that life is contingent on death (dead organic matter feeding live organic matter). The metabolic relationship between live and dead matter is therefore similar to the entropic relationship between hot and cold whereby one state is both categorically (in terms of what is) and dynamically (in terms of how) contingent on its opposite, forming a dialectic pair. Therefore, the Socratic statement that life is contingent on death and vice versa just as heat is contingent on cold, can be valid only on the condition - that it is kept on the observable physical plane. On that plane, however, the eternal element, as chemists and physicists would attest, is matter - that which passes from one state to another without ever being lost - and not rationality. Socrates makes a poetic shift in a rational claim when, using the observable instances as a trampoline, he tries to leap beyond the observable universe, claiming that Life is contingent on Death. Death not as a state of matter ("for surely the act of dying is visible" (Plato, Ibid.) but as a state of consciousness, as the domain beyond the sensible, as Socrates puts it:

"That soul, I say, herself invisible, departs to the invisible world to the divine and immortal and rational." (Plato, Ibid.) 
The soul, that which had animated the body, is now gone - it has disappeared from the sensible world the way only information can disappear. Laying inert is a body that now fully belongs to the empirical, mechanical, bio-chemical universe.

"Well... let us suppose that there are two sorts of existences, one seen, the other unseen... The seen is the changing, and the unseen is the unchanging... the unchanging things you can only perceive with the mind - they are invisible and are not seen" (Plato, Ibid.)

So, the conceptually thinkable, according to Socrates, belongs to the domain of the invisible which is the domain of death. Suddenly, the one truly rationally unknown, Death, is flooded by the very rationality that cannot reach it. For Socrates, Death is the door through which rationality escapes the sensible world of change.

If we agree that the "seen" refers to the entire world of appearance, the world of form, be it physical or mental, sensible or logical, then we can agree that it is also the world of change. All form, from a fleeting vision of a water drop to the most enduring concept, is subject to temporality, to change. From the fast-paced phantom of thought or the slow-moving shape of inert stone - this is the range of the moving image, the world of image as always already in movement. Anything beyond the phenomenal world, beyond form, beyond the threshold of death, would presumably be unchanging. Positing the unknown as unchanging is a mytho-poetic leap that we can intuit in the notion of existence as such, of all that ever is, or of the stability of our singular perspective (the singularity of my experiencing the world, regardless of what the experience is in particular). However, to attribute reason to the unchanging, "invisible world to the divine and immortal and rational," is to attribute a tool of human knowing to the unknown an obvious confusion that seems to have entangled generations of subsequent rationalists in the nets of mytho-poetic certainty. 
This discussion of Socrates with his students at his last hour unravels in a dream-like setting - when his students arrive at the start of the scene, Socrates tells them about a dream, at the end of the scene he drifts into eternal sleep. The wakeful scene is an allusion to the Platonic idea that life is a dream (later elaborated by Descartes and his methodology of doubt) from which death would awake us. This notion is fortified by the suggestion that dream is a command of the reality that extends beyond the terrestrial dream - Socrates tells his students about a recurring dream that he has been following all his life but only now, in his final hour, has literally and simply obeyed. The dream has been a prompting to make and cultivate music which he had believed meant philosophy as "the the noblest and the best of music," until now when finally "I thought that I should be safer if I satisfied the scruple, and, in obedience to the dream, composed a few verses before I departed.” (Plato (2) 2009 [360 BC])

The poetry of dream entering through the widening door to death in Socrates final hour, joins the invisible certainty of rationality in a dance macabre that beckons Socrates in the realm of thoughts when the body is threatened with extinction. The disembodied life of thought - was it Socrates' premonition of the afterlife or about cinema?

My first encounter with death at the age of four forced me to face the destabilizing intuition that I can be no more. The terror of this realization was eventually offset by the idea of the unknown - death was nothing but an unknown. Sure, my body would die, but since I was still not fully entrenched in my body and it seemed to be changing (everyone telling me I had grown up since they last saw me, etc.), I didn't identify too strongly with it. Obviously my body was in the process of becoming which I felt nothing of since all relevant information about it came from the outside - measurements of my size or exclamations of adults upon seeing me. Thus my living body and the point-of-view awareness I identified with were not inseparable or even concurrent, neither did they seem or feel contingent on one another. My awareness was me and my body was 
something assigned to me that I was in the process of discovering from the inside, so to speak, as a feeling, as much as others were in the process of observing from the outside, as a changing object. The adult perception, which is also the one that implicitly permeates disciplines is that life and awareness belong to the same order as they both generate themselves or, in other words, express intentionality, move from within. However, it is perfectly possible to conceive (and indeed that is another childhood intuition) of inanimate matter possessing silent awareness. So, although they are related by way of intentionality, the connection between life and awareness is conjectural - we have no way of knowing, from the position of biological bodies who are aware, whether the two are intrinsically or only circumstantially related. Plato brings this conjecture to the fore and demolishes it with the intuitive certainty of mytho-poetic thought. It still roots its arguments in intuitive certainties but it severs those certainties and destabilizes them in rational fragmentation. What happens if I sever my intuitive certainty that I am not my body from the intuitive uncertainty that what I experience as "I" may be fully contingent on my body? What happens if I sever the sense of continuous unbreakable Now, the sense of immortality that being conscious entails, from the fear of death encoded in my constantly threatened by change and fragility body? What happens is a bipolar schism that, when multiplied and manifested in society at large, gives rise to dogma - the religious belief that there is life (i.e. Awareness) after death and, conversely, the scientific presumption that life and awareness end at the point of physical death. (1) Finally, what happens is the hypnotic lull of cinema.

Cinema carries over the landscape of thought in the luminous realm of repetitive permanence - a disembodied life lived at the gate of dream and death as simulated by the dark cave of the movie theatre and the projected window of the movie screen. A womb, a cave, the body of Nut - this is where knowledge emerges from the ecstatic fornication of celestial light and terrestrial mass. This is the original setting of the cinema - a setting of communion pregnant with 
all the dangers and possibilities that such an augmentation of symbolic reality could entail, from revelation to zombification.

The domain of the seen and the visible, the domain of Bergsonian images, so to speak is always already in movement - whether paced by millennia, like the tectonic shifts, or by instances as in the speed of thought, images are always already moving. However, that expanse of images is available to us at different speeds and thorough different temporalities. They are sometimes available to our perceptions first, and at others to our reason, sometimes they can be only grasped poetically, and others they lend themselves to quantification and description. Cinema can bring all those temporalities together flattening them all on one temporal field - that of the speed of technological projection.

"By close-ups of the things around us, by focusing on hidden details of familiar objects, by exploring commonplace milieus under the ingenious guidance of the camera, the film, on the one hand, extends our comprehension of the necessities which rule our lives; on the other hand it manages to assure us of an immense field of action. Our taverns and our metropolitan streets, our offices and our furnished rooms, our railroad stations and our factories appeared to have us locked up hopelessly. Then came the film and burst this prison-world asunder by the dynamite of the tenth of a second, so that now, in the midst of its far-flung ruins and debris, we calmly and adventurously go travelling." (Horkheimer \& Benjamin 202 [1947], 236)

Technological projection is a form of reflection that brings all kinds of appearances to the same plane and creates a labyrinth of illusions, reminiscent of Plato's myhto-poetic reasoning. The cinematic manifold maps so thoroughly the human experiential field that it has obfuscated its reflectivity. However, in order to understand it as technology, we should begin at its inception. 


\section{The Mirror}

"And God said: 'Let us make man in our image, after our likeness; and let them have dominion over the fish of the sea, and over the fowl of the air, and over the cattle, and over all the earth, and over every creeping thing that creepeth upon the earth'."

Genesis 1:26

And Man said: 'Let us make technology in our image, after our likeness; and let them have dominion over the fish of the sea, and over the fowl of the air, and over the cattle, and over every creeping thing that creepeth upon the earth'. It seems only natural that the image reflection would inadvertently reflect the gestures of its source and try to be reflected in turn. This perpetuates a maze of reflections potentially obfuscating the way to the original light source.

The mirror is perhaps one of the most ancient human inventions - the first known mirror dates back from 6200 B.C. Like its first known Ancient Egyptian counterparts (4500 B.C.), it was made of polished stone. The Ancient Egyptian mirror was associated, not exclusively, with the goddess of beauty, fertility and love Hathor, also known as the Eye of Ra. A magic eye was often painted in the centre of Egyptian mirrors probably referring to Hathor but more importantly, implying that the mirror was looking-back at the onlooker. The mirror as a perceptual opening appears in divination practices across cultures. The Hebrews and Ancient Greeks consulted the surface of still waters while the Toltecs believed a mirror held under water would turn into an opening through which beings from another realm could appear and even pass to our world. The Egyptian word for mirror, ankh-en-maa-her roughly translated meant 'life-force-for-seeing-theface' and the hieroglyph for ankh (life) looked like an oval mirror with a handle. Eternal life was contingent on the preservation of ka, the life-form, believed to be sustained by mirrors placed in 
the tombs for transition in the after-life. In short, for the ancients, the mirror was not a mere symbol of vision, truth, light or life, but the main tool of elaborate technologies to cross into other realms. The mirror was a channel, a portal, an opening to knowledge, much more akin to the direct knowledge enabled by mirror neurons that allow us to undergo an experience of an action by observing that action as performed by another. The mirror neurons do not reflect back but absorb the reflection as direct knowledge.

"When the mirror is dim, the soul is unclean" states a Japanese proverb. Socrates advised young men to consult the mirror to determine their principle path in life according to the beauty or ugliness of the image they saw. But for such a look in the mirror, one had to have a sober eye. We know what happened to Narcissus when he laid his cursed eye on his reflection. The story of the mirror is as much a story of the technology as it is a story of the human eye. How did man look and what did he see? The ancients seem to have looked at the mirror less than they have looked into it - peeping behind the reflective surface to receive messages from the unseen. Whenever they allowed their eye to be locked at the surface, turning the reflective surface into an optical tool, they seem to have been forever imprisoned by their reflections, as cautioned by the tale of Narcissus.

When in 1841 Feuerbach turned his mind's reflective telescope toward God, man had already flattened the mirror to an optical tool, its sacred connotations reduced to vulgar superstitions. In the Essence of Christianity, Feuerbach argues an understanding of God as a reflection of human aspiration, a sort of augmented human image freed of its characteristic limitations. (Feuerbach 2008 [1841]) Henceforth, the idea that man was not created in the image of God but rather God was created in the image of man would take an increasing hold of the collective imagination and search confirmation in technological reflections, launching the cultural maze of mirrors that Western thought would increasingly dwell in. 
The English word mirror is etymologically linked to miracle through the Latin mirrari (to wonder at). The Latin mirrari turned into the Old French mirer (to look at) somewhere in the 11th century. By that time, at least in one part of Western Europe, the reflected image ceased to be a source of wonder, an apparition of sorts, and became a mere technical appearance on a surface. This shift from the open act of 'wonder' to the optical close-circuit of 'looking at' coincided with the Great Schism of the Christian Church (1054 A.D.). Hannah Arendt tracks the loss of contemplation of being to the modern ideal of thinking and acting back to Galileo's gesture of turning his telescope to the skies. This single gesture, she argues, validated Cartesian doubt as the cornerstone of modern epistemology, whereby the ancient thaumzein (the wonder of seeing) as linked to theoria (the still contemplation in wait for revelation) was replaced by the active notion of thinking which called man forth to hunt for the truths ordinarily denied to his senses.

But a closer examination reveals that theoria was lost long before Galileo's historical gesture. Theoria comes from Greek theos (god) and oros (seeing) and can be roughly translated as 'seeing god'. It signified the silent contemplation in view to god's revelation to nous (inner eye). The word retained this significance in Eastern Orthodox theology referring to the contemplative practice potentially leading to theosis (union with God) as an abstract goal, but the predominant Western interpretation comes from another Ancient Greek view of theory as speculation. Euclid (aprx. 325-256 BC) derived the term theorema (proposition to be proven) from theoria. Theoria was also linked to theoros (spectator). As the meaning of spectatorship would shift, so would the meaning of 'seeing by way of contemplation'. Theoria thus had the ancient potential reading of speculation as well as contemplation. Scholasticism as a method of philosophical training based on Plato's teachings began in the early church, but in the 9th century the method was reformed in Latin Christianity (what would become the Roman Catholic Church) placing exclusive emphasis on the dialectical approach. After the Great Schism, Scholasticism was instituted as a Roman 
Catholic system that opened up to Aristotelian logic and developed its specific brand of Christian Rationalism. Reason and dialectics became critical tools in approaching theological questions. Thus the Great Schism roughly finalized a gradual parting of the methodological ways between Eastern and Western Christianity. By the 17th century, theory had long lost its intuitive revelatory aspect in dominant Roman Catholic practices of learning. This defined the cognitive context of Galileo's empirical advancement of the Copernican system.

In other words, as mirrari (wonder) turned into mirer (to look at), contemplation (inviting revelation through open questioning) turned into speculation (looking at closed propositions). 'Seeing through' was replaced by 'looking at' in Western syntax. This semantic flattening of the field of vision, was the precursor that would eventually turn the mirror from a window to a surface. The Eye of Ra would be shut and we would be left only with the reflective surface bouncing our image back at us.

But this shift, like Arendt's Archimedean point, had its blueprint in the rational dreams of ancient Greeks. Even before the Platonic fission of the world in seen and unseen, from the Pythagorean vision of a mathematical reality reflecting divine reasoning to Parmenides' trust in reason beyond the illusion of the senses, the ideal of a rational universe had already begun to establish itself as the cornerstone of a new syntax of mediate knowledge. Of all human ways reason was the one that held the most technological promise (as the practice of the linear way of language), exemplified by the Pythagorean trust in mathematics as much as by Parmenides' trust in logical propositions. Reason as a sequential mode of interpretation allowed for incremental corroboration of reality that could be objectified through demonstration, stored and transmitted without relying on the capricious vagueness of non-linear revelation and individual predilection. The distance of language imbued reason with the promise that it could exists independently. The clash of poiesis and reason was evident in the Plato's efforts to clarify the former through the 
latter in the discussed passage of Phaedo. Plato was thinking on the juncture of this shift from open questioning to closed propositioning, when technology was turning from an opening to an appropriation. The opening to the unknown was a way of knowing through a state of surrender, while the appropriation of the known was a state of control, of taking arms against the unknown in a dream to conquer it. Marcuse's technology as war (Marcuse 2004 [1998]) was thus embedded in the syntagma (2) of reason. Significantly, Archimedes became not only the visionary of a point outside Earth, but arguably the first to use reflection, the mirror, as a weapon. The original significance of technology as providing tools of both care (culture) and dispute (war) as a way of communion and communication with the environment is exemplified in the development of the mirror from a miraculous opening to other realities to a reflective weapon.

The use of the mirror's reflective properties for war marked a turning point in the semantic flattening of the tool. To Arendt's Archimedean point, one could then easily add an Archimedean reflection - a reflection that figuratively and literally bounces off the surface of a mediate object which can then redirect it in any given direction. The physical distancing from the Earth epitomized by Archimedes' lever is complemented by the mental distancing technologically epitomized by the mirror allowing the point-of-view awareness to become aware of itself from an "outside," as an object. The reflected image confronts the onlooker with a new type of selfknowledge - the knowledge of a self-recognizing-oneself as another, the beginning of conceptual distancing in what Damasio calls extended consciousness:

"...extended consciousness is the precious consequence of two enabling contributions: First, the ability to learn and thus retain records of myriad experiences, previously known by the power of core consciousness. Second, the ability to reactivate those records in such a way that, as objects, they, too, can generate "a sense of self knowing," and thus be known." (Damasio 1999, 197)

This type of knowing is a historical and spatial knowing - the travelling that conceptual language makes possible in order to observe and cover otherwise inaccessible perspectives. 
Contemplation, open questioning, is akin to staring at an object of enquiry directly, without the safety of a mediate object that could mitigate the potential blinding effects of the revelation as dramatized in Plato's Cave. Conceptual language in particular and reason in general, through offering themselves as the reflective tools of distancing, promised enlightening glimpses of the world outside without leaving the security of the Cave. Thus armed with the reflective shield, Western thought would gradually lose its fear of the unknown, marching on with a growing sense of invincibility.

But this new-found invincibility had a new set of dangers, broadly cast out in what would be Meno's response to Socrates - if the questioner in a Socratic dialogue knows the answer, then the questioning won't reveal anything new, if he doesn't know it, then he has no way to ascertain whether the given answer is correct. The solipsistic danger was deflected by Socrates with the concept of latent knowledge. He would henceforth view himself as the midwife assisting the appearance of this latent knowledge through questioning. However, the flattening effect of reflection obfuscates depth so that such delivery would not be possible without the contemplative trust in the unknown as unmanifest.

By instituting Scholasticism as a method of theological reasoning based on reflective dialectics and Euclidean theoria, Roman Catholicism implicitly surrendered theological mystery to rational control. The symbiosis of reason and conceptual language defined by linearity could not fathom ubiquity. Since the mirror of reason could only be cast at one angle at a time, the danger of entrapment in the sequential ways of its method or even worse, in the perceived supremacy of an angle, became ever so pertinent. God could not be both immanent and transcendental at the same time just like a reasonable man could not be both inside and outside a house. Feuerbach's argument was already implicitly manifest in official Western Christendom. 
Henceforth, Western cultural syntax would exponentially define itself as a language of reason. Reason would no longer be one mode of interpretation, one cognitive approach, it would be cognition itself. The language of reason, the sequential approach so to speak, would, by the distancing it affords, open the possibility to become an object of itself, to becoming another item of the mapping of knowledge. However, this opening also contained the danger of solipsistic entrapment in syntax, threatening to obfuscate the very notion of the unknown, as already implied in Meno's argument. This threatened poetic thought in new ways. When Plato saw the plastic arts as imitations of God's reflection in natural phenomena, he postulated a techno-scientific imposition on the field. Seeing objects of art as imitations meant regarding them exclusively as objects, as closed descriptive sentences. Like the mirror which was closing itself as a window to another realm, the artwork was set on its way to becoming a surface. The image of God was already on its way to optical reduction. Therefore, by the time Christianity captured the European imagination, the latter was already impregnated with the seeds of techno-science, of Archimedean reflection. The Hebrew divination by gazing at still waters was about to meet the Narcissistic stare. How hard it must have been for the latter to conceive of itself as the image of God in nonoptical terms? From there to the identity photography the road was winding but the arrival was a matter of historical time. Identification as knowledge would become the backbone of rational enquiry. Identification in terms of categorical language is thus interpolated with optical recognition.

"In the beginning God created the heaven and the earth.

Now the earth was unformed and void, and darkness was upon the face of the deep; and the spirit of God hovered over the face of the waters."

Genesis 1:1 
This is the first biblical account of God after whose image Christian man was created. (3) In the beginning God's image had not yet emerged from the primordial darkness. A darkness implicitly referring to a realm beyond the finitude of creation. In this account, before the creation of light, God is referred to as spirit hovering over the non-reflective waters. Beyond the first day of creation there is a mystery outside linguistic intent - that initial silent will is the source of creation. The Bible does not verbalize negative existentials, or rather does not venture to render a realm beyond rendering - just silently refers to it by omission. But conceptual reasoning cannot grasp realities outside its language and since I am conducting this questioning within the parameters of reason, I shall honour its syntax by stating the paradox that in the Biblical account a nothing that is real, i.e. is like something, abides outside creation. God, an emanation of this nothingness into something-ness, is therefore partaking into that nothingness before the emanation takes place. But to state anything 'before' is to stretch time outside its inception. So, to grasp the first two verses of Genesis one has to step outside time not only as we know it (which is the time brought into being later in the account) but also in its primordial sense, as we don't know it - a task utterly unfathomable within the syntax of reason. This brief and not exhaustive detour is only to outline the gulf between mytho-poetic and rational textuality, and the absurdity of the idea that reason alone could tackle the questions defining mystic cognitive systems. How can 2-D straight line describe a twisted 3-D doughnut without losing its objective in a myriad of crosssections?

On day four God calls into being celestial bodies and puts into motion the markers of time as we know it. It is only after that moment, on day sixth, that God's image emerges in the making of man. This image, this initial form or blueprint, is neither the totality of God nor the full potentiality of man - the Bible proceeds to tell the story of the image's unravelling through its complex relationship with the divine, from the deceptive appropriation of knowledge in the 
Garden of Eden to the potential of salvation in the grace of the Holy Ghost. Time itself seems to unravel at different speeds throughout this pre-cinematic tale - from the compact explosions of the first six days to the progressively shortening life-span of God's image - the initially immortal human that eventually arrives, after a couple of capital punishments (the expulsion form the garden and the great flood) at the modern post-flood life-span of 100 years.

The expulsion from Eden focuses on the moment man claims something that is not given him - knowledge of good and evil. That particular type of knowledge dooms man to working the land, to extracting its fruits, not merely receiving them. The knowledge that holds the promise to liberate man from his formal predicament, i.e. to 'becoming like God', also holds the curse of labour and work. If the linguistic intent that God uses to bring the world into being can be called a natural technology in the broadest sense of technology as a means to an end, then human language is the first technology that emerges in that appropriation. Language not merely as communication but as appropriation of knowledge and more importantly, as a form of intending. To tap into language as intent, a form of calling into being outside time-space as we know it, man had to do a fantastic, if not impossible, feat - to overcome the limitations of his form, the limitations of the image so to speak. Trapped into his own image, in a world of pre-existing forms where language as intent holds no sway, man is subject to the danger of language as wilful thinking, of technology as a means of labour.

This Hebrew post-Eden rendering of humanity as technologically determined was introduced to European thought at a cognitive juncture that was on the way of divorcing conceptual language and poetic knowledge, thus truncating the possibility of addressing the entire first day of Genesis, i.e. the mytho-poetic guideline to the full scope of language as intent, as an opening to a realm outside language altogether. The ramifications of this truncation were monumental. 
If the mirror of reason could only be used one angle at a time to assist the examination of all aspects of a phenomenon, then the critical moment would be the gathering of those angles into a cohesive whole. But how could the postulation of infinity of mappable knowledge be curved into a bound object? The mere accumulation of incremental knowledge wouldn't behave as Comte had hoped. In order to gain a uniting view of the pieces, one had to step outside, to push to an even greater distance from that which examined so as to encompass the entire region. The constant necessity of more distancing and new perspectives is not only contingent on space but also demands an expansion of space, not as an expansion but as an increasingly complex structure. It calls for shifting from the descriptive mode and, in searching for more leverage points, to venture into new layers of assembly that are only indirectly accessible to the human faculties - incredibly, quantum space becomes accessible only when enough cosmic distance is achieved to make spatial infinities actively imaginable. From there to hyperspace, the leap is only a matter of a formula - the conceptual way to poeticize space-time. This exponential distancing however, endangers the view of the original ground, threatening to sever reflective language from the body of the terrain altogether. Moreover, it contains the danger of mistaking the distance between two perspectives on the ground for the distance between the ground and the encompassing third angle view. The history of Western thought is more often than not the history of the fight between such perspectives. But it is also the history of mental abstraction that allowed for an imaginary outside point, an Archimedean point, to gather those angles in a mental space unfettered by perceptual limitations. The Archimedean point of cinema.

The invention of the lens is obscured in antiquity, but it was the union of the two old tools, the mirror and the lens, that first transported the eye outside its natural field of vision. Although the reflective telescope was not the one used by Galileo, the next few decades would see it upgraded in the hands of Newton - the man who solidified the Archimedean point by 
cracking the mystery of gravity itself. The path from there to the light-weight giant piezoelectric mirror technology developed by NASA for use in space telescopes (News Editorial 2000) was only a matter of history.

The Industrial Revolution catalysed mirror-making with cheaper methods that allowed for mass production. Now that the window-in-the-mirror was closed and alien beings had no access to the rational world, the Narcissistic stare, mitigated by the utility of a mechanical Socratic consultation, would soon become quotidian practice; facing the abstracted image - a daily communion with the self. The reflecting surface would allow every man not only to see himself daily, but also from angles only imagined before - a privilege of the affluent few until then. Reflective thought, the linguistic legacy of Echo, would finally join her beloved Narcissus trapped in the technological reflection of the mirror, in a techno-linguistic collaboration both combustible and never quite satisfied, never quite unified. Conceptual techno-language became the mental framework of scientists and laymen alike. In an ironic twist of Cartesian duality, the body and the mind alike were semantically flattened in the reflective surface of the mirror. It seems that man had succumbed to the internal pressure of the Cartesian split by collapsing both his immanence and his transcendence into manageable flat surfaces reflecting into each other ad infinitum. The unknown was thus deflected by an ever receding infinity. There were no more shadows in Plato's cave, only life-like reflections from an ever increasing number of mirrors - a veritable maze of optical illusions extending the perceived walls of the cave way beyond any imaginable outside. It meant that one day humans could no longer keep track of the trajectories of those myriad reflections, and the amplified directional light rays might meet and combust, blowing out the cave - the very platform of security in the name of which man had appropriated the mirror in the first place. And this is what cinema did, it "burst this prison-world asunder by the dynamite of the tenth of a second," (Benjamin 1936) reflected the site and captured it in to a 
recording that could travel and bring near that which is distant - the nearing of the distancing of conceptual thought cinema turned into a way of experiencing. The mirror image was liberated from the subject and set into flight:

"The feeling of strangeness that overcomes the actor before the camera, as Pirandello describes it, is basically of the same kind as the estrangement felt before one's own image in the mirror. But now the reflected image has become separable, transportable. And where is it transported?" (Benjamin 1936)

The floating image, liberated from the host of its original reflection, and thus dislodged from embodiment by the Archimedean lever of cinema, can now live forever and wherever after.

\section{NOTES TO CHAPTER 4:}

1. Here I do not refer to the domain of religion or science, but to the collective convictions extracted from those domains, inevitably crude and simplistic, addressing a childhood insecurity that makes collective messages more widely comprehensible, communicable and hypnotically convincing.

2. Syntagma can be roughly translated from Greek as constitution; I use this term tentatively to indicate a cohesive body of rules and principles that can be likened to a meta-paradigm.

3. In the Bulgarian translation of the Bible, the word used for the creation of man is more akin to 'bringing forth'. Strikingly and tellingly, the equivalent word, an intermediary between creation and making, is not to be found in the current English language. The Bulgarian translation therefore accounts for a subtlety of the degree of creation from the initial gesture of creation through the constructive process that follows in Genesis. To address the intricacies of the creative process (in view to language and cultural translation) is a matter of a whole separate investigation. Suffice to say, that my use of the word 'creation' henceforth, i.e. creation of man as officially translated in English, is only conditional in view to keeping within the focus of the current argument. 


\section{Chapter 5. Art: Diffractions of Technology and the Body}

The intent for the cinematic liberation of reflection from the body already transpires in language as a means of travel. The symbolic way of language as a way of thinking with language forges a detachment from the givens of the moment. Through the symbolic we can travel away from ourselves; through the conceptual way of the symbolic, we can organize that travel in spacetime, the body remaining only a point of reference. By contrast, in perception "we understand ourselves not as having but as being bodies." (Carman 1999, 208) Thus the Cartesian self, the "me" that thinks itself as "having a body" is in cognitive dissonance with the one who is perceiving as a body. Language objectification conflicts with experience in ways that interfere with coherent understanding - a conflict that can find resolution in artistic production where the symbolic and the sensory are contingent on each other, and even more actively, in cinematic experience where the convergence of the symbolic and the sensory are not only brought together in a work, but also played out in a life-like simulation. Conceptual travelling becomes a sensory experience in cinema. Being a perceiving body is not compromised by the mental detachment necessary for travelling in language. The techno-scientific viewers, the citizens of the rational universe, no longer have to "rank the credibility of their own bodies about as low as the credibility of the ocular evidence that "the earth stands still," and thus, apparently, allowing with complacency their securest possession to escape (for what does one at present believe in more firmly than in one's body?)", (Nietzsche 2003 [1886], 40) Their bodies remain intact as they travel with the film which is no longer a mere extension of their bodies - it becomes an investment of and in their bodies, a body substitute that stands alone and merges with the viewer in an incestuous communion. 
This techno-human amalgamation is the final destination of a movement away from ancient bestiality. The launch of rationalism in continental Greece was itself made possible only after the defeat of the Cretan Minotaur - the product of an interspecies romance between the queen of Crete Pasiphae and a white bull gifted to her husband Minos by Poseidon. When the Athenian Theseus defeated the Minotaur, it was not only Athens that was liberated by Cretan dominion, the entire Western culture was liberated from the confusions that baffled that strangest of all animals - the symbolic beast, the human. The memory of this most troubling time where the possible, the imaginable and the natural were entangled in a world of cross-species and human sacrifice lived on in Greek Theatre, through the most cruel Coliseum punishments to the Spanish corrida, still playing out the drama of the human facing bestiality. The intensification of technological development in the $20^{\text {th }}$ century traumatized the collective identity and set the conditions for a return of bestiality in 70 s action performances and in the inauguration of cinematic pornography - a true platonic beast. The techno-human condition began to redefine the ways of being with, altering and violating the body. Two of its more influential incarnations appeared in the theories of Donna Haraway and Jean-Francois Lyotard that shed light on the collective anxieties about the body and death.

For both Haraway and Lyotard the apocalypse (the ultimate mortality of the known) or alternatively, solar death (Lyotard) is a way to think technology; a way that points to a future of hybridization with radically different connotations. For Haraway (1991), a propagator of the language games Lyotard pronounces as his methodology, the apocalypse is a patriarchal narrative instantly outstaged by her "myth" of the cyborg as "post-gender" and therefore without origin story - an amalgam of partial identities without beginning and end (ironically, this dramatic statement uncritically presumes that beginnings/ends are by definition gendered, i.e. it perpetuates (its own interpretation of) patriarchal meta-narratives). In other words, the cyborg is the promise 
of liberation from categorization (identity generalization) and respectively, fears of (identity) fragmentation inscribed in totalizing (patriarchal) myth/theory. It is humanity's way to take responsibility for its intimate relationship with technology. Haraway infuses the cyborg with libidinal valence that allows her to envision a timelessly pleasurable experiencing of what would be interpreted as nightmarish monstrosity within the patriarchal matrix. Indeed, Haraway's "myth" turns the orgiastic Dionysian plunge into chaos on its head, blending techno-scientific construction with the Dionysian absolute permissiveness. It is as though, with her cyborg, Haraway has conjured the offspring of Dionysian bestialities - the Minotaurs of human mating with technology. But while in Nietzsche's ancient Greece, individuality is impossible in preEuripidean tragedy where such bestiality is possible (in the Dionysian mystery it is lost to the eschatological elements) in Haraway's post-gender universe, the joyful marriage of human and technology, guarantees the proliferation of fractured identities as a way to resolve the threat of identity loss. In other words, to Nietzsche's dynamic unity of the Apollonian and Dionysian instincts, Haraway opposes a physical amalgamation of their respective amputated (or rather, halved) versions, under the claim that only partial identities are lived identities. This empirical, contra-Platonic grounding in the lived uniqueness of identity (ever so slightly deviating from the ideal model of itself) is itself fused in methodological matrimony with Haraway's textual reliance on identity/myth construction. This vision of the cyborg carries the inevitable implication that the body is by default deficient (or threatened with inevitable deficiency that should be compensated) and that the only way to resolve this deficiency (i.e. to empower the body) is by coupling it with technology or rather, by investing it in technology, by instituting and imposing as condition of its liberation (from a certain social configuration) its constant alienation and mediation (into another social configuration). In order to subvert "organic wholes," Haraway proposes hybrid wholes that destabilize the notion of biology as implicitly claiming pre-discursive content. The notion of the 
techno-biological hybrid replaces the unknown with the unimagined. This destabilization of the organic, along with the placement of the agency in the mental as empirically applied by industrial technologies, lays bare an implicit destination of conceptual distancing. The distancing emancipates the concept from the body to a degree of disconnect - the free-floating concept, invested with the mass-energy of technology, is no longer an extension of the body but its partner, its incestuous mate, and ultimately, its substitute (as foregrounded in the dream of the fully thinking machine). The offspring of that union however can be as unpredictable as any other Dyonisian project placing the power of emergence in the technological domain.

Lyotard (1991) investigates the possible investment of the body in technology by making an imaginative leap to the solar end (with the respective impending annihilation of life). Death as a point of departure for making sense of techno-scientific utopia. According to Lyotard's sobering view, language-thought is only the mediating "mnemonic" software of an organic "hardware" that is the human body. And although it may be simple to imagine a solution of constructing alternative hardware that can survive conditions in open space outside our solar system to support the software of language-thought beyond the solar death, such project is not only logistically farfetched, but principally inconceivable. At this point of understanding artificial and organic intelligence, the two are non-interchangeable as the latter does not operate on binary code and is intricately related to perceptual (hardware) experience (i.e. it is not merely supported by its hardware but interwoven in it). In other words, the ultimate hybridization of human (mind) and technology (body) is contingent on problems and paradoxes, the possible solutions of which (if any) may lie entirely outside the reach of the techno-scientific paradigm.

Lyotard foregrounds the capacity of techno-science to easily lend itself as a totalizing tool for the protection of the "social whole." Thus, he does not call for an assault on "traditional" theory but rather for the systematic questioning of theoretical knowledge. This questioning itself 
becomes a sort of anti-totalitarian guarantee which can only be effective through a dramatic approach away from synthesis and reconciliation, a sort of non-Hegelian dialogism reminiscent of Nietzschean pluralism and, although supportive of Marxist critical approach, weary of its regulatory potential (this attitude is also echoed in Haraway's reservations to Marxists tendency to exclude divergent positions):

"And since for Nietzsche "the general character of the world is eternal chaos," "will to power," and "a play of forces and force waves," therefore the Text of the world should also be "read" in a pluralist way, excluding the generalization that could lead to dominance of a single interpretation. In this sense, Nietzsche's pronouncement of "God's death" should not be interpreted exclusively through religion. This "death" and this "parting with God," as Lyotard would later say, is also a parting with the "great narratives."' (Zhabilova 2010)

So, it could be said that Lyotard's weariness of synthesis carried forth a Nietzschean intent unfolding in the conditions of techno-scientific reflection. In a society where grand narratives have collapsed at the advent of new technologies, Lyotard attributes the danger of unification to functional positivist knowledge related to techno-scientific production itself. The unity of a product, any product is always already subverted by its incompleteness. However, rather than destabilizing the technological agenda, a perpetually unfinished product refines the thrust to infinite production Arendt sees as characteristic of the technological imperative that searches to replace work with labour. This would mean the exclusion of the slow-paced care from the relationship of the human to technology, to the interest of impersonal machine speed. The activity of labour and the consumption of its fruits, which have come to dominate the public sphere of such a society, cannot furnish a common world where humans might pursue their higher ends. Labour and its effects are inherently impermanent and perishable, exhausted as they are consumed, and so do not possess the qualities of quasi-permanence which are necessary for a shared environment and common heritage which endures between people and across time. In 
industrial modernity there is a reversal of values that displaces the traditional values that define the work of homo fabricans with those of the labour of homo laborans - product is replaced by process, permanence by abundance, the world is elbowed by the value of endless life itself. (Arendt 1958)

The thought of a machine-dominated environment is not alien to Lyotard's attention that does not see humans as the inventors of technology. Through his lens, the principles of technology (as part of poiesis) already animate the biological world where life is a technique operated by the genetic code (as a kind of language). But this language does not allow the objectification and controlled complexification of the respective technology; it operates by breaching (i.e. poetically) and not by remembering (i.e. linearly) as human language does. Accumulation and development is the contemporary programme that no longer has mankind as its objective (i.e. it has surpassed the Enlightenment project) and is now dedicated solely to differentiation, a process contingent on mediation between two related elements. The mediation of this relation allows for alienation and modulation (two of the corollaries of Haraway's cyborg utopia) and is the domain of techno-language or technologos (Lyotard). Language being, unlike genetic code, infinitely cumulative within the limitation of its inscription, i.e. a sort of linear cognitive infinity, is therefore an auto-techne, a technology that works on itself, the implied condition for techno-science and modern technology (reminiscent of Compte's vision of science as an infinite accumulation of incremental knowledge). The (temporally) infinite horizon or openendedness of technologos, allows for the revealing of what has been previously excluded from it and the invention of new linguistic genres like arithmetic as well as the construction of science as a cognitive discipline. However, the process of conquering the unknown and codifying it for memory leads to experimentation and complexification - this entire process of linear development Lyotard calls scanning and sees as the driving force behind techno-science (perhaps 
it would be worth noting the kinship of Lyotard's idea of infinite scanning with Arendt's vision of endless proliferation following the value reversal of contemplation and action, and ultimately of world and life). Since scanning does not have the reactive regulatory mechanism specific to breaching (i.e. the technological (or auto-poetic) dynamic of life), it goes on exponentially, collapsing the very notion of finality and introducing new levels of complexity that not only turns humanity into a vehicle of its process but demands of it dehumanization in order to fit its telegraphic modality.

However, as already discussed, Lyotard sees this call for hybridization, for fusion with the machine so to speak, as an impossible task that can only indefinitely terrorize the bio-cultural human with its totalizing challenge. Working openly within the Heideggerian tradition on technology, here Lyotard expands on the notion of new technology as challenging to nature and enframing to humanity in a totalizing mobilization toward dehumanization. In other words, although Haraway's myth of the cyborg is rooted in Lyotard's vision of techno-scientific development, the two theorists have diametrically opposing views on the ramifications of that development - where one sees liberation, the other foretells oppression.

The concept of scanning is also central to Lyotard's critique of the (Platonic/Freudian) notion of representation (mimesis) in art. As a form of technologos, art is effectuated by scanning as free flow of energy - a primary process that knows no negation and is only thrown into chaos when met with the rigid structures of secondary organizations, e.g. representation. Chaos is not a primary state; it becomes chaos in confrontation (or polemos) with order. Understanding art as representation disrupts scanning and misdirects attention from what is standing as concentration of libidinal energy to a pre-supposed referent outside the work of art, thus failing to harness the primary energies concentrated on the libidinal surface of the visible. The repression of structured boundaries, of embodied identity, according to Lyotard, happens in the artistic process as the 
artist lowers the theoretical boundaries between exterior and interior, allowing the free energetic flow beyond the body of the artist. Artist and art-work dissolve into one libidinal surface contingent on the repression of structure (a process akin to an active (post-linguistic) return to the primary shimmering field). This energetic fusion does not call for the hybridizing re-structuring of Haraway's cyborgs perpetually suspended in fragmented yet structural unity. It could be speculated that in Haraway's world (despite the efforts of Stelarc e.g.) there is no possibility of experiencing art, at least not in the sense Lyotard understands it.

In both her cyborg mythology and her analysis, Haraway systematically aestheticizes science, effectuating a functional version of the Marx-Engels' view of art as a tool to (mimetically) synthesize and communicate to the masses scientific and "polit-scientific" information. The contrast with Lyotard's mistrust of representation goes further when considering his positioning of the sublime in this post-modern choreography of interpretations. Unlike the techno-scientist swept by the forward mobility of technologos, Lyotard endows the artist and the writer with agency within technologos, i.e. with authorship of the rules of their work. As individuals engaged in a private process, they necessarily produce micro-narratives, thus avoiding the techno-scientific danger of generalization. Lyotard relates to Heidegger's trust in art as the saving grace of technology. He sees this saving grace not only in art's capacity to generate micronarratives but also in its capacity to invoke that Dionysian norm-lessness of experience. When there are no rules or given procedures for what is different (as per the technological imperative to differentiation) within a discourse, there is a disruption of linguistic flow - a silence that is a break, an impossibility of conjuring language. This moment of silence is marked by what Lyotard calls the differend, which signals the experience of the sublime in conceptual repression. The differend is thus without an object, beyond representation. The experience of the sublime is an experience of the collapse of the relationship of subject-object. It derives from the Kantian 
subjectification of the sublime as the experience of the incapacity to find object-ive match to an internal state (rather than the incapacity to respond to an object) but goes further in identifying the state as a form of collapse of inside/outside, object/subject differentiation rather than a state of implied inadequacy. This collapse of language into the differend is reminiscent of the collapse of order into the Dionysian ecstasy, in other words, the collapse of identity and surrender of agency to the indefinable, the unintelligible, to the poetic leap. What is specific to the differend is that it is by default incommunicable to all thought, it cannot be universalized; it is the guarantee of freedom from totalitarian imposition and the productive mission of the artist. The invocation of art's capacity to provoke cognitive/experiential immediacy is, according to Lyotard, qualitatively different from the hypnotic effect of representation that inevitably promotes meta-narratives as inscribed into the structuring of (referential) language. Therefore, Haraway's cyborg myth itself offers one more potential meta-narrative that, within its beginningless/endless story line promises the technological perpetuation of difference.

While Lyotard's artist offers the possibility of trans-subjectivity, Haraway's cyborg cannot promise more than the intersubjectivity of "respect for the other" (species). This means the cyborg, as a structural unification of different species, would inevitably remain alienated to parts of itself (symptomatic of Marx's industrial alienation) in a triumph of techno-science that has instituted that alienation as absolute (partial identities). Implicit to this vision is the terror of silence, of structural collapse, of death; in its fear of the incommunicable, it has tried to conquer the Dionysian by referencing it in the imagery of hybridization - a surgical transplantation (as opposed to synthesis) of the Dionysian onto the Apollonian. The terror of death perpetuating the terror of a totalizing process (technology's version of life) ad infinitum. Atrophied by industrial production bodies/identities are deemed inadequate and in (emergent) ontological need of compensatory augmentation: by hybridization they are permanently attached to an-other that can 
thus guarantee the involuntary policing of possible transcendence beyond those closely guarded given structural boundaries. The doors to Lyotardian sublimation are thus firmly sealed, the proliferation of the techno-scientific mindset ensured.

Haraway proposes the cyborg myth as a gesture of feminist liberation. But how far the supposition that the hybridization of human and technology (Haraway's trans-human leap) guarantees agency can sustain its own "lived experience"? To the post-gender world of Haraway, Lyotard offers the gendered body as a challenge to thought. This challenge is encapsulated in the old epistemological paradox of how could one (having only the tools of constructed thought at one's disposal) know whether gender difference is ontological if ontology implies a state beyond construction? A challenge that Haraway evades by deploying the capacity of language to annul empirical reality by addressing the "knowability" of the construction of "ontology." But rather than a subliminal explosion (that would fulfil the other end of an eschatological narrative of the dying sun), Lyotard, disappointed, observes that the paradox of the gendered body generates infinite thought and challenges the possibility of (mental) exodus from the impending solar death (i.e. from the final frontier of apocalyptic meta-narratives):

"As a material ensemble, the human body hinders the separability of this intelligence, hinders its exile and therefore survival.” (Lyotard 1991, 22)

How can those deliberately irreconcilable views, the view of the sexually transgressive cyborg breaking the speed boundaries of death by reinventing and reinvesting the body as a mechanized thought and the view that the structureless body as a libidinal vortex of thought unto death be reconciled, not just with each other but with anything else they are trying to assault, including themselves? The thought made flesh in post-apocalyptic technology, the aesthetic of the scientific, the edge of rational mirroring meets the libidinal flow of bodies made boundless by the poetic breakthrough of the sublime in the movement of technology and poetry in cinema. The 
explosion of the world in the endless spatio-temporal transgressions of cinema (Benjamin) is just such a technological monster as it intermingles with the perceptual fabric of the viewer lowering the boundaries of subject-object in the cinematic amalgamation between seen and seer, dream and dreamer. The cinematic time-space explodes into poetic timelessness only when this technological potential is delivered not only mediumatically but also by way of film language, foregrounding the importance of film art as the way of cinema that accounts for both poetry and rationality.

According to Lyotard, lack can only exist in structural relationships, with the subliminal collapse of the structural; pleasure and pain become only affirmative energies. Conversely, his proposition could also imply the subliminal potential of the structural collision of pleasure and pain as constructs of excess and lack. The most graphic and most stylized illustration of this principle at work would be the S\&M situation as the site of intensified libidinal energy. But intensified energy is power and it contains the danger of Foucauldian power dynamic (play) as much (if not more) as it offers a subliminal opportunity. And while discourse may be theorized as the principle social mediator of power, it is its relationship to new technologies as conceptual materializations that allows us to take a closer look at power's contemporary incarnation.

Enlightenment's project to free humanity of power (over) relationships by removing God as the ultimate Other failed not because it couldn't manage the latter but because its understanding of those relationships was not adequate to the objective of the project. So, instead of an outpour of freed energy, the missing Other opened a hole, an energetic vacuum that was filled by technoscience - a product of humanity that, the latter fancied, would remain in a power under position by virtue of being its brainchild; a fable inversely mirroring humanity's libidinal relationship to God in a startlingly close resemblance to the arch of the Uranus-Saturn myth. The ensuing anxiety of technology taking over man the way man has taken over God was thus not a paranoid 
fantasy but a threat contained in the compositional fabric of cultural time, or perhaps, as a Freudian might theorize, a self-fulfilling guilt prophesy.

This coup automatically prepared the levelling of the gendered field - man and woman no longer needed to compete for paternal affection, they could now parent technology in a new partnership that might also afford them redemption through the embassy of woman, theorized as another Other who, as such, could only have been indirectly, if at all, implicated in the act of the coup. The gendered body was thus emancipated to respectful difference (re: Haraway's intesubjectivity) generating Lyotard's infinite thought. It was no longer the subliminal other Lyotard fantasized about, but more than that - inversely intuited in the encounter of Adam and Eve. That encounter was closed and vindicated by absolving woman from the divine coup; what remained of the subliminal encounter between man and woman was sexuality as a function of the body, beyond the biological imperative, a representational description - a pure pornographic movement unfolding on the antiseptic screen of cinema. Man and woman were no longer the lack of each other, they had invested that lack in an offspring - techno-science - that promised to compensate all their inadequacies, to bring them to their full potential, to realize their dreams and extend their power ad infinitum while they copulated in a barren endless loop of electric light.

When Wim Delvoye talks about his sculptural installation Rose of Wind: North, East, West and South (a variation of the Into Me/Out of Me piece, 1992), he refers to the line connecting the mouth to the anus (a line that he more recently explores again in Cloaca, 2006 and in Anal Kisses, 2000) as epitomizing the cruelty of the body. The body that Artaud offered at the stake of his theatre of cruelty in the hopes that it would survive the structural collapse by becoming what it is - Lyotard's pure boundless energy flow abolishing the viewer-spectator differentiation in a moment of communal sublimation. Standing for the absent performer, the four male figures of Delvoye's installation point their mouths (both filled up and opened up by the 
telescope each one holds) to the sky while their jut-out anuses offer a sticking-out microscope to the viewer. The four figures guard the four cardinal points like the keepers of the gates between the macro and micro-cosmos - a ritual invocation of the viewer to peep into the universe through the anal microscope protruding like a second penis (Delvoye) that both threatens to penetrate the eye and invites it to penetrate the body: a crossing of boundaries promising to grant the eye a ray of the universe captured by the telescopic mouth. The specular anus is enabled by the new optical technologies that both allow perceptual feats and shut down the senses entirely. In doing this, they become the indispensable mediator between the universe and the viewer (God and humanity) using the human body as its hard drive, rather than (potentially) offering themselves as the hard drive of humanity. Haraway's cyborg is coming as an incestuous fantasy of consumption and waste, as life after life.

The anus - the last frontier of the discoursed body is the last carnal secret to be unveiled, the last orifice to be salvaged from the abysmal connotations of its ocular obscurity. Delvoye notes that his fascination with the anus is rooted in his childhood questioning of the fact that the televised hero never went to the toilet. The anus as the only body part covered in silence by public discourse, defecating the only body function publicly unspoken. We are bound to question the anus, but more important is the question - why now, why the anus? It is tempting to follow the obvious relation between consumerism and the anus as the dark end, the exit, of the human metabolic complex. In an era of heightened environmental anxieties, addressing the anus may promise to resolve that collective bodily fixation which has translated into environmental imbalance. But what is of more interest to the current investigation is the anus not as a metabolic but as an energetic frontier. The difference between the two may seem a matter of semantics, but it is much more than that - the metabolic is the discoursed energetic of the body. The abysmal connotations of the anus are of interest here as they invoke the last Dionysian frontier, the last 
locus of elemental obscurity that may be systematically mobilized to subliminally collide with the Nietzschean Apollonian in order to produce the artistic differend that would guarantee an energetic exit from the power hold of structured reality. The opening to the cave of the body, the source of dark energy, the window to the stars.

Ron Athey's Solar Anus (1998-2000) performs the theme of the subliminal collision of the anus with the universe, of the holiest and the most profane in discourse. (The profanation of the anus may be just a corollary of the bourgeois discursive mapping of the body, but this is a conjecture that calls for investigation. Suffice to say that the anal kiss as the perceived basest (and thus most self-negating) of gestures was instituted as a core element of La Vey's Church of Satan initiation ritual, thus presaging its deployment in body art). Inspired by Georges Bataille's essay of the same name, Ron Athey's performance redeems the anus by making it the centre of a black sun that erupts pearls and light (as opposed to feces). Delvoye's techno-human body as the (hybrid) gate to cosmic light is hailed from the other end of the hybrid spectrum as the fusion of human and cosmic bodies. Without the tele-microscope, the anus emits its own celestial light.

The absence of new technologies from Ron Athey's work resonates in their unapologetic use in Wim Delvoye's Cloaca (where the latter developed and constructed his own elaborate shitproducing machine - a complete replacement of the cruel metabolic axis mouth-anus). However, both artists seem to be themselves part of one axis that channels the same problematic from two different directions - the absence of new technologies in Ron Athey's work by omission relates to them as does their presence in Wim Delvoye's work. Ron Athey's Dionysian invocations sound like incantations against techno-scientific fecundity as Wim Delvoye brings the latter into satirical collision with its shadow - carnal waste.

Perhaps it could be argued that, by mobilizing the anus for subliminal collision, artists also render it vulnerable to the illuminating rays of (Apollonian) discourse. This would be so if 
the positivist dream of a world entirely knowable by the accumulation of incremental description or conversely, the deconstructionist vision of a world entirely contained within and contingent on that description were indeed possible. But the only phenomenon that remains irreversible, unrepeatable and incommunicable within that description, the bona fide Other of every human as well as of everything else that is conjured up within the cognitive economy of human thought Death - remains an ever-present challenge to an Apollonian project disconnected from the cave, as death is nothing but a Dionysian event, the erupting energies of an ever-dying god. So, the Platonic cleansing of the carnal can perhaps only intensify the inevitable touch of death.

Pleasure and pain may be only affirmative energies when structural boundaries are repressed, but, within those boundaries, they remain the functional signals of life and death. From the point of view of the structural drive, the collapse of boundaries is death, therefore their repression is suicidal. Pain would be the last signalling frequency an Apollonian society would tolerate - this statement would be all Ernst Junger would need in order to assess the subject of such society.

Contemporary of the social intensification of new war and media technologies between the two world wars, Ernst Junger witnessed the sedative power-hold that the industrial dynamic had on society. The promise of convenience new technologies systematically fulfilled was instrumental to the power dynamic that held people hostage to the idea of security. Pleasure and safety insinuated victory over pain by constantly deferring it in a society full of commodities that acted as deflecting mirrors. Held in suspended animation by the lull of this power grid of convenient objects, the human was disabled. The way out of this situation Junger saw in the mobilization of the human body for confronting rather than evading pain. To the consumer culture that produced hedonistic subjects (individuals who focused on the body as the site of pleasure) he offered the heroic martyr type who would objectify his own body as the space through which pain 
is experienced and thus master pain through detachment rather than avoid it through denial. In the endless differentiation/individualization Junger saw a strategy of industrial technologies to render the human benign. His call for disciplining a social type instead would condition detachment and the capacity of (Dionysian) dissolution of the self. This would guarantee the reinvestment of power in the human body that, thus reconfigured, would be able to integrate and withstand pain in the "realms of life where [it] reigns as absolute master."(Junger 2008, 16)

Junger's objectification of life by technology (later showing in Arendt's vision of life as technological process) is thus countered by the auto-objectification of the individual body. The visceral commitment to this solution of the industrial techno-human imbalance, whether theoretically articulated or not, is shared by all body artists that, interestingly, have emerged as socio-cultural phenomena after WWII. The body as a military zone itself, as a space of discipline, would prove a fertile artistic enterprise. The subjective objectification of the body, a form of distancing awareness that redeploys conceptual strategies back into the original site of embodiment, would not only enable the redeployment and articulation of pain (e.g. the Vienna Action Group) but also re-frame the articulation of pleasure itself (e.g. Carolee Schneeman).

Junger's new worker type ready and able to embark into the domain of pain, i.e. in proximity to death, would also enter into a new relationship with the new technologies. This new relationship would take ownership of their enframing and would allow the human to work with those technologies symbiotically, but through a position of detached awareness, not detached permissiveness. The human torpedo (intuiting the Japanese WWII kamikaze) would thus incorporate its sentient component as a willing extension of the deadly machine. What Junger is interested in is not military efficiency but military aesthetics as a strategy of human empowering, of conquering its secret desires and fears that cloud the cognitive eye (Junger 2008, 8). While Haraway sedates the fear of technology by marginalizing the fear of death through the endless 
cyborg, the suicidal hybrid of Junger does exactly the reverse - embraces the fear of technology in order to reinstate death in the fabric of life.

Reminiscent of a cross between Allan Ginsburg's carnal poetry and Peter Greenaway's operatic visuals, Ron Athey's performances condition the stage to sustain the repeated collision between the sacred and the profane placing the performer on the threshold of pleasure and death. The artist's direct reference to classical depictions of religious martyrdom and concurrent use of S\&M props and techniques in works like 4 Scenes in a Harsh Life (1995) charges pain with a new valence. It is not only the pain of the martyr or even the pleasurized pain of the martyr, but the martyrdom of sexualized pain. Athey's self-flagellation is not invoking the submission of Jesus to paternal sacrificial will, but rather the voluntary, almost arbitrary sacrifice, a desperate offering to an ever receding God that is not asking for sacrifice, a silent indifferent God, lost in the Enlightenment coup. Sexualisation is not used to guarantee the libidinal clash of pleasure and pain (that much could be extrapolated about any form of ecstatic pain that often characterizes martyrdom) - its display and identification is inevitable, it is the only form of intelligibly articulating that type of libidinal clash in a society where every form of pleasure and pain is discursively mapped and categorized. In Athey's performances the spectacle of this collision is essential just as it is essential in Junger's military display enabling the objectification of the body, its conversion into a site of transcendence, a spec in the energy flow.

However Junger's spectacle, although lavish, is strictly organized. It represses the boundaries of individuation through formal unification in contrast to the Dionysian mystery that relaxes the boundaries of individuation through chaos. Artaud's theatre revives the spectacle of this chaos within the performative parameters of the mise-en-scene - the obvious lineage of Athey's work. But, like Athey and Delvoye, Junger and Artaud guard the two ends of the same 
cognitive-experiential axis described on one hand by those who search the solution to the technohuman conundrum through new technology and those who completely ignore it.

To the visual cruelty of Artaudian shock, Junger offers the specular cruelty of new media, the eye of the camera as reflective of the eye of the new human - an evil appropriating rapacious eye that captures the world and not only alienates it by extracting its image, collapsing its space, but also alienates the self from oneself. This cinematic way of replaying the world (as opposed to the theatrical staging) is not without its promise. It would cultivate the detachment necessary to discipline the body so as the proximity of death feels secure - a state of security that gives back humanity the agency taken away by the marginalization of pain. The new human would then be capable to offer that body to whatever lies beyond its boundaries, to that nameless unrepresentable other that may be the dethroned father, the ever-dying Dionysus or simply death, without fear or expectation, as a free sacrifice to whatever transcends the boundaries of identity.

If there were no discernible sadness in Junger's vision of techno-human society, he could have infused his highly organized war spectacles with laughter - the domain of Apollo that, according to Artaud, has also been lost at the advent of modernity (Artaud 1958 [1938]). Perhaps the determinate seriousness of the techno-scientific project cannot afford to dissipate energy in either tragedy or comedy, in either tears or laughter, in either pleasure or pain - all those require a certain level of being present, a certain economy of attention that would trigger a counterCartesian doubt that perhaps the body does exist, that all the narratives about discourse and macro and micro, and grand coups, and impending danger don't count for anything if they don't account for the touch of death - the ultimate leveller of all boundaries.

The cruel line that connects the mouth and the anus is also the line that cuts through the body in order to sustain it. It is an elongated opening that renders it imbalanced, metabolically open and dependent on outside forces; this is the line that prevents it from closing up and 
whirling away in solitary self-sufficiency, but also the line that guarantees that it never entirely gives in to the illusion of a permanent differentiation invincible to terminal blows. The two ends of this line are guarded by artists still searching for differends; Ron Athey at one end trying to redeem the act of Saturnine usurpation by refusing to partake in the parenting of technology and Wim Delvoye, in turn, trying to articulate this cosmic joke with all the tools at his disposal as humour is perhaps the only form of communication that can afford anything.

The advent of industrial technologies generated environmental shifts that had the character of cognitive floods. The new technologies were changing the very matrix of social space-time thus shattering cognitive paradigms, not just epistemological themes and narratives. This development is evidenced in the intellectual mobilization of artists and thinkers in late 19th century to question and re-examine cognitive assumptions. From Kant's phenomenology to Cezanne's tonality, the industrial human was focusing on the intricate ways that gathered and organized information into knowledge more than at any other time in history.

The Impressionist breakthrough in visual arts was a response to the claim of new optical technologies (photography) over representation. Objective visibility became the domain of the photo lens (significantly called "obektiv", i.e. Photo lens, in Slavic languages) so painting focused on exploring and fortifying that part of vision that could not be reduced to the new industrial medium - the subjective apprehension of the visible, the phenomenology of visual perception. By the 30s the urgency for understanding of the new industry-driven syntax of mediated time-space intensified and abandoned its defensive character. Perhaps because of the latter, most artistic developments of the period were interpreted as a celebration of the times, but just like Junger's attempt to contain the flood by opting for a wilful symbiosis with new technologies in an aesthetics of death was hastily politicized, Modern artists were not so much swept by the flood as they were working on developing strategies to understand and harness its 
power. Through (spatial) multiplication and (temporal) acceleration Industrial technology had tapped into the very faculties of space and time that allowed for their lived conversion (and the emergence of Einsteinian space-time). This also meant the breakdown of continuity, the advancement of a new spatio-temporal dynamic matrix that gave unfair advantage to the new technological imperative over the human senses. It was the principles of that matrix that Modern artists began to explore. The new matrix meant a new cognitive syntax that would be fortified by the advancement of industrial media into mass media. Where industrial transportation hit a spatiotemporal wall it could not overcome - (the speed of) light - the media overcame by simply abandoning the physical, the body, and extracting only its lightweight signature (sound and image), i.e what could be directly liquefied into language.

By the time Marshall McLuhan began writing his theory, the media of new technology had already permeated the everyday and launched its global grid, i.e. the waters of the flood had settled and soaked into the very fabric of society - the everyday. Stepping on the shoulders of Modernist writers and artists, he began to unravel that grid in order to bring the underlying principles of media communication to consciousness. His effort in communication was congruent with the phenomenological project to tap into awareness (without having to translate it to Freud's meta-figuration). What Merleau-Ponty did for human perception and the body, McLuhan was doing for the media messages as ways of acting on perception and the body.

From his first book, The Mechanical Bride (1951), to his last, Laws of Media (1988), McLuhan does not deviate from this project, starting with printed advertisement and working relentlessly through all media and its multiple communication layers. His systematic reference to Modern literature and art however is not merely a side effect of his professional background in literature. The Modernists worked from the privileged moment of being contemporary to the "seismic shift" of new technologies, when the new matrix had not yet become the given of the 
televised world. They had witnessed the collapse of old givens and had worked out the syntax of new constructs. By the time of The Mechanical Bride, this syntax would have already become a given:

"Industrial man is not unlike the turtle that is quite blind to the beauty of the shell which it has grown on its back... He grows the shell unwittingly, subhumanly, biologically..." (McLuhan $2002[1951], 4)$

McLuhan's analysis not only transcribes the beauty of the shell for the reader, but also, by appropriating its aesthetic, attempts to invoke its perceptual effect, to move the reader to a fullview vantage point. To the psychoanalytic unconscious or the phenomenological pre-reflective (Merleau-Ponty) as a space of resolution of the empirico-rational cognitive paradox of how can the unknown be recognized as known, McLuhan adds textual organization, the syntactical foundation of media/modern communication, as the space of potential cognitive agency. Like Merleau-Ponty's pre-reflective body always whole and relational at the same time, i.e. always in a subject/object state that allows for knowledge to both reveal itself from the outside and be reflected and disambiguated from the inside (Merleau-Ponty 2002 [1945]), McLuhan's textual platform (the carrier of information to the enquiring body) is also pre-reflective in the sense that it is the irreducible given of new media dynamic but also in the sense that when unveiled and brought to consciousness, it opens to the enquiring body, engaging it in co-authorship of the message.

Discontinuity, shifting of angles and mosaic composition characterize McLuhan's textuality. This approach not only generates poetic openings that demand reader participation in order to connect the parts of the mosaic but also, in a bona fide Nietzschean way, addresses the practical "need to use as many views and positions as possible to get a clear over-all sense" (McLuhan 2002, 70) in order to keep in step and respond adequately to the "shifting imagery of 
our society." That shifting imagery would later mature in the concept of the all-at-onceness of the acoustic space McLuhan coined as the space of new technological society. Non-homogenous and multi-perceptual, this was a transformational space that allowed for the newspapers to be "bathed in" not merely read. According to McLuhan, the acoustic space was brought to social attention by Picasso's cubism where the return of the simultaneous triumphed in the multiple viewpoints that put the eye in many places, indeed used the eye as other senses, inviting the viewer, or rather demanding that the viewer finished the picture by connecting the multiple angles, by making sense of its disjointed mosaic.

But that space was also transcoded by the French symbolists, followed by James Joyce in Ulysses, who "saw that there was a new art form of universal scope present in the technical layout of the modern newspaper." (McLuhan 2002,4) That layout is the familiar front page mosaic of information snippets brought together from all over the world, from different angles and in different tonality, like a cubist collage. To McLuhan the technique of news gathering that brings together disparate bits of information from different parts of the world, overlaying them in the same page like a work of jazz is of special interest because it produces the non-homogenous space that overwrites the news themselves with its underlying message of human solidarity. This observation, made in the first pages of his first book, is the precursor of McLuhan's signature "global village" and "the medium is the message."

While McLuhan focuses on composition and spatio-temporal organization in the traditional (hot) media of print and painting, like in the case of the multi-sensory eye of Picasso, he finds the same organizational effect happening one layer deeper in the new medium of television. With television, this same synesthetic demand is made at the level of the medium itself where the eye is called to function like ear, taking up many visual bits and composing them in the mind, a sort of temporal mosaic. Television as a cool medium, relying on hearing for broad 
sensory mobilization, works on a deeper unconscious level that hardly allows agency or coauthorship. McLuhan's mistrust of this visceral effect of the television medium could hardly be construed as technological enthusiasm. His work was as much about transcoding media as it was about finding a balanced relationship toward media ecology. Like Junger, he was unsettled by the sedative effect of new technologies and the passivity that cool media produced. Between Junger's human extensions to the machine (the human torpedo) and McLuhan's media as extensions of human faculties there is a short step and a long nuanced dialogue.

The title of The Mechanical Bride refers to the theme of sex and technology (the axis sextechnology-death is also the one McLuhan establishes as central to the new folklore) where industrial man is seen as a puppet subjected to the technologically conditioned specialization and fragmentation of the body exemplified by "the legs on pedestal" advertising trend. The subtitle of the chapter of the same name ("The Mechanical Bride") uses parataxis to convey this corporeal fragmentation:

"The walk," "the legs," "the body," "the hips," "the look," "the lips." (McLuhan 2002 [1951], 98)

Reminiscent of Pound's "Eyes, dreams, lips, and the night goes," this line is only one literal example of McLuhan's stylistic references to Modern literature. It is both an illustration of fragmentation and an invitation to appropriate fragmentation through language in order to turn it into a cognitive agent instead of allow it to become a tyrannical pressure effect the new media grid exercises over the body. Parataxis is also only one facet of McLuhan's interest in discontinuity and fragmentation in communication. A significant part of Laws of Media is composed like modern poetry of theory (Notebooks) while the entire Mechanical Bride is a collage of seemingly unrelated chapters. From the sentence, through the phrase to the full composition, McLuhan relies on principles of discontinuity echoing Modern painterly, musical 
and literary techniques and harnessing the fragmenting imperative of the techno-scientific project for poetic impact. To the discontinuity of The Mechanical Bride there is a certain oneiric resonance. The advertisements sound like the collective daydream of industrial man, but they also acquire a certain nightmarish quality as they follow relentlessly one after another, short and intense, like a hopeless waking up in endless consecutive dreams, somewhat reminiscent of Finnegan's Wake (1939).

On another level, when regarded as separate works, each advertisement becomes an object of meditative focus - McLuhan unravels not only the manipulative intention behind but also the mindset and the socio-technological conditions behind that intention. He patiently tracks the circuit of conditions-conditioning agents-conditions as a spiral spreading a single pattern, a single mindset, as wide as possible (and he doesn't spare effort to track how wide). This intense inspection of a moment of consciousness-forming perception, of an advertisement as a capsule of formative consciousness, of a single micro-event in all its nuanced detail, recalls Proust's unwavering eye.

The apparent lack of composition of the chapters in relation to one another starts to fade under a sense of another type of continuity, the polysemic continuity of poetry where disparate elements are organized according to principles underlying literal connectivity. Reinforcing the compositional mosaic are the questions/subtitles in the beginning of each chapter the answers of which are not overtly offered within the body of the chapter - instead, they suggest information about the structure of the problem being discussed, referring to information that is either outside of the immediate text or between its layers of organization. In addition to the semantic organization of the chapters that becomes clear upon closer inspection, the first and last chapter of the book have either been inspired by or directly referencing to the beginning and end of Ulysses. The latter begins with a scene that could be said is as much about whatever happens in it 
as it is about a mirror and ends with a stream of consciousness about male/female. Likewise, The Mechanical Bride begins with a chapter about the front page newspaper layout which is held like a mirror, a medium of self-reflection, to the times in question and their specific heterogeneous modality; it ends with a chapter on horse opera and soap opera as the media rendition/mythologizing of the conditioned male/female collective unconscious.

And somewhere in the middle of this McLuhan simply sings out: "I'll mow you down!" Truculence, jaunty irreverence, dandified elegance, light-hearted lies, and pathetic boastfulness, mounted on a bubble of illusion..." (McLuhan 2002 [1951], 16) How else should one refer to dandified elegance but in a melodic tone?

The acoustic space is a return of the tribal, but not because history has short-circuited. Industrial technologies have launched a new cycle and we are at its tribal, should we say prehistoric, stage. This idea is yet another point of conversion with Picasso whose interest in prehistoric art was certainly a little more than mere colonial exotic fetishism. A collapse of visual absolute Euclidean space, acoustic space is described by discontinuity and simultaneity.

"In acoustic space, which includes the dynamic co-action of the figure as part of its basis, everything produces its own space, i.e. it forms the basis, even when it is formed by the basis." (McLuhan 1992, 39)

Artistic co-authorship is the rendering conscious of the dynamic of this space that reciprocates the pre-reflective body. The conscious enactment of this principle through the cognitive discipline of arts/communication is implied by McLuhan as the opportunity (within the danger posed by new technology) to save humanity from the tyranny of new media. But this salvation is not one of evasion and avoidance - it is by walking right through the electrified field of media. McLuhan's mosaic does not merely draw inspiration from Modern literature - by adopting the laconic fragmented style of product advertisements and film editing, it foregrounds 
the aesthetic economy brought forth by new technologies with their demand for speed, production turn over and sound-bites. The cinematic splicing of the chapters in the Mechanical Bride both underlines the dangers presented in the new technological formatting and, at the same time, pushes its poetic capacity to the very edge of comprehensibility, demonstrating a new aesthetic that, like Vertov's cinema, would keep the engagement of the reader in a tensional bind that precludes unconscious surrender to the technological formatting. 


\section{Chapter 6. The Body of Cinematic Technology}

Cinema is a capture of the moving image - a taxidermic endeavour. It skins reality and preserves its image in the formaldehyde of celluloid, magnetic tape or digital support. By making the sensible world abstract and recordable, cinematic technology opens up the possibility to bring the rational project to its glorious endless conclusion - to circuit back and bring to the senses that which rational abstraction would have initially taken away. The cold greedy camera intruding on the sexual act of a pornography shoot brings back the image to the consumer in the warmth and intimacy of his/her private space. The original libidinal act is now a play button away, with minimal energetic investment required. And while the pornography of today is a strictly audiovisual experience, in Metzinger's universe where technology and science rush ahead to transcribe a reality of mere signals projected as representations in the brain, a future where cinema is not a technology of the moving image but that of a moving representation. A future where a holographic projection that responds to touch and induces tactile sensations is almost here. It is not farfetched to imagine the pornographic scene becoming a fully sensual interactive experience, as close to the encounter of flesh-and-blood people as electricity would allow. Cinema is not about its medium or language; it is not about cinema, just as the essence of technology is not about technology (Heidegger).

We don't create technology, technology creates us (Lyotard). Or rather, we are locked in a reflective loop where we perpetuate the technology that perpetuates us for reasons that surpass both us and technology. Conceptual thought presents an offering - a distancing and detachment from the agglutinating force of interpretations and within this offering lies the danger of dislocation and detachment, of death by dismemberment. Technology brings the gift of conceptual thought to sensible reality and, as such, its power becomes power over life itself, a 
power of construction and destruction. Cinema synthesizes the movements of technology into a gift or a glove for the senses.

When Peter Greenaway announced that cinema was dead, he pointed at the introduction of the remote control in 1983 as the technological event that ushered the advent of interactivity. In the same breath he optimistically exclaimed that "new electronic filmmaking means the potential for expanding the notions of cinema have become very rich indeed." (Coonan 2007)

But how can cinema die when it has no body? Cinema only comes through its tools; it does not coincide with them. The birth of cinema as a field of production can be assigned to the period between 1893 and 1895 when the movie camera/projector were brought about by a series of uncoordinated inventions. In other words, cinema is historically born by the invention of a single equipment technology. However, aesthetically it is mobilized by the pre-cinematic motion picture, dreams and wakeful images of reflections - captives of chronological irreversibility:

"In those days the world of mirrors and the world of men were not, as they are now, cut off from each other. They were, besides, quite different: neither beings nor colours nor shapes were the same. Both kingdoms, the specular and the human, lived in harmony; you could come and go through mirrors. One night the mirror people invaded the earth. Their power was great, but at the end of bloody warfare the magic arts of the Yellow Emperor prevailed. He repulsed the invaders, imprisoned them in their mirrors, and forced on them the task of repeating, as though in a kind of dream, all the actions of men. He stripped them of their power and of their forms and reduced them to mere slavish reflections. Nonetheless, a day will come when the magic spell will be shaken off." (Borges 2005 [1957], 18-19)

This day may have arrived with the invention of the movie equipment that allowed for the moving image to be recorded on film, replayed and modified independently of its corporeal original. The new mirror, the film stock, offered a new type of captivity - the film reflections were no longer "slavish" repetitions of man's actions, but independent images imprinted in a potentially infinite space-time. Like text, they could be read and re-read, playing out their trajectories over 
and over, regardless of the embodied space-time of their prototypes. But how this disembodiment, this skinning of physical reality, is brought to practice?

"In order for the productive body to reveal itself, the biological body must be dissected, viewed in parts. Its lost unity can only be retrieved epistemologically, while also accounting for the division of labour, separation of task. Productive activity has to be extracted from the body itself, from living labour, and relocated within the parcelization of physical motion, which is most meaningful and at its most efficient when an organ is attributed a specific and unique function as a guarantee of its infallibility. Yes, it is included in a general mechanistic plan whose meaning is not entirely understood by the actor-participant and which, as it is carried out, leaves a mark on the body - as in Menenius Agrippa's fable, by allowing the individual to portray himself metonymically, through an image in which a fragment of the body becomes the entire body." (Deleule 1992, 208)

Traditional artistic technique is based on the transmission of such contiguity unto the body of work. The metonymic reduction of the biological body in the artistic production process rebounds in the emergence of the art work as a unified body. This is to say that, as the entire body of the painter is gathered in the hand, becomes the hand (extended into the paint brush) so to speak, it brings forth an art work, a painting that is whole in itself, a vision unified by the body of the painting. In other words, there is a libidinal transfer from the body of the artist to the body of the art work that necessarily passes through corporeal metonymic reduction.

The technology of the traditional arts, the bringing forth of the work of art so to speak, is eventuated by the gathering of technique and equipment into a mediumatic language. For example, the equipment of painting - brushes, canvas, tripod - use the medium of paint and the platform of canvas to build up the painting - the embodiment of an image that appears in and is contingent on this mediumatic embodiment. So, in a way, the libidinal transfer from the artist to 
the artwork is manifested in meaning. In traditional art forms, the relationship of technology to both the art work and the artist's body is categorically different from that in new (Industrial and post-Industrial) media art forms. In music as in painting and, most significantly, in theatre, there is no single piece of equipment that defines the entire technology, or that is irreducible to the human body. One can use one's fingers instead of paint brush, one's vocal cords instead of a musical instrument, etc. Dance is even more organic in that its medium is the body itself. In all art forms, language is defined by the body-bound medium, i.e. visual language, body language, sound language and the amalgamation of all those in the language of performance. In literature, the only disembodied traditional art form (and arguably, the precursor of new media) the medium is language itself which only through writing, has been abstracted from its original embodiment in the spoken word.

Language as a symbolic gathering of concept-signs that have no contiguity with their signifieds is unlike image which coincides with corporeal identity. Words have no intrinsic similarity to the concepts they encode. This nominal relation of language to embodiment enables the easy transference from the spoken to the recorded/written. In other words, writing is not to language what image is to body. The written and the spoken word "apple" have the same type of signature relationship with a physical apple - they both lack any qualitative similarity with it. The word (spoken and written) is only a sign encoding the concept of an apple. By contrast, an image of an apple is an abstraction of a physical quality of the apple - it is its visual (sensory) signature. The sensory languages of audio-visual art forms, as based on abstraction, are qualitatively different from language as such on one end, and from the embodied language of dance and performance, on the other. Unlike language, which has a nominal relationship to physicality, body language is physicality in motion, it is necessarily embodied and embedded in physicality and cannot be libidinally transferred through another medium. Until the technologies of mechanical 
recording came along with the promise of neuroscientific decoding of the sensory make up of Metzinger's ego tunnel.

Therefore, abstracting the corporeally embedded moving image as language is a process of more complex dis-agglutination. Unlike traditional art-forms, the instrument of molding the medium of the camera is irreducible to the eye that it mediates. This is to say that the eye cannot substitute the camera the way the hand can substitute the paint brush or the voice - the musical instrument. As already discussed, the camera is an independent body that can, by contrast, substitute the eye in the capacity to potentially gather its material regardless of any immediate human intervention, as in Warhol's Sleep (1963) where a camera is set to record John Giorno sleeping for five hours and 20 minutes. Once again we are reminded that the camera "developed in response to a crisis of sensation, as a cognitive tool that served as a prosthetic for vision." (Elder 2010,4) This prosthetic is not a mere extension of what is already there, the way a paint brush is. It fills in the place of a missing organ or limb, of a lack that has been identified in the given state of the body. The body is no longer a state of grace bur a state of limitation set free, or rather ignored, by the invention of the camera. However, the filmmaker can forgo the camera by manipulating the film strip directly thus, in turn, ignoring and making obsolete the camera equipment, the very technology that inaugurated cinema.

"In the case of hand-painted film, the film strip is not (or is no longer) the place of automatic impression of light and colour; quite the contrary, it is the receptacle, the place of inscription of a purely manual gesture." [Translation mine] (Belloi, 2006)

However, this embodied film inscription becomes quickly redeployed by the cinematic apparatus with its contingency on the cinema projector or broadcast equipment. Moreover, with the advent of new media, it becomes hijacked by computer technologies which offer a full range of tools, often surpassing the traditional ones and always providing a reversibility of the 
production process that brings film-time (visual reversal) into the fabric of active production (with functions like "delete" and history palettes). Thus, the technological character of cinema is not merely a matter of specific equipment; it is reflected in the language of cinema which is always already accommodated more efficiently by industrial and post-industrial media, in an ever spiralling competition between the human touch and the technological processing.

What emerges in this questioning is the twofold disembodiment in cinema - the disembodiment of the work process and the disembodiment of the work. With film the image is recorded onto the medium but this recording is not the work itself. The work is present only insofar as it is projected. Light is thus the actualizing medium of cinema. It carries the reflection away from the immanence of the reflective surface, liberating both image and movement (i.e. time as trajectory) from both original and mediumatic embodiment. The image recorded on the film stock was divorced from its object (which happened already in photography) but also divorced from any subsequent immanence such as the immanence of the static physical photograph. While the photograph is still a form of presence or object/body, film is only an apparition, captured and replayed over and over again, like a memory that one does not need to remember. The body of cinema is a body made of light. The body-light is possible when its physical support is invested in any technology that can render it - film, video, digital formats. The advent of television and later of cybernetic technologies allowed for new types of projections (e.g. the broadcast) and started to displace the film projector. Decasia (2002) as the film that draws attention to the physical life of the film material as evidenced in the unique unrepeatable process of decay, of dying demystifies the eternal incorruptible glow of the body-light of cinema, yet by being a film, it renders that very death of film eternally re-playable. It also marks the industrial shift from film to digital technologies in an almost funerary way. The film medium has become so marginalized since 2002 that major production facilities and labs have shut down. 
Cinema as a metaphor of life is suggested in the very names given to its two major branches - animation and live-action. But what does life without embodiment mean? To most of historical humanity it would mean life after death, to the modern human it means cinema. This may account for the hypnotic sway that cinema holds over the popular imagination, but more than that, it exemplifies the process of reversal of world/contemplation and life/action Hannah Arendt identifies as "the point of departure for the whole modern development", whereby Western society gives away the amori mundi (as a world of things) for the value of life as a process, an automatic functioning where "individual life had actually been submerged in the over-all life process of the species". (Arendt 1998 [1958], 321) Arendt trusted that the liberation from the enframing effect of this value emphasis on life-process would come through the singularity of the individual artist. If cinema is indeed the dominant artistic manifestation (and an agent) of this enframing, then its release from the industrial imperative would be through the subordination of the production process to individual artistic making. This would mean opening up the reflective surface of film back to that which lies beyond through poetry and dream.

Dream is where the beings from the other side of Borges's mirror come back, crossing the boundaries of the reflective surface that has trapped them into a mimesis of human action or conversely, which has trapped humans to constantly reflect back on their reflections. To talk about dream means to talk about the dissolution of wakeful consciousness into random apparitions. If the solipsism of the mirror reflection connotes the close-circuit of reason and selfconsciousness, dream is where that close-circuit is broken and language spills chaotic, fluid and ever-evading the categorization that gathers singular identity as seen by Lacan:

"This jubilant assumption of his mirror-image by the little man, at the infants stage, still sunk in his motor incapacity and nursling dependency, would seem to exhibit in an exemplary situation the symbolic matrix in which the $I$ is precipitated in a primordial form, before it is objectified in the dialectic of 
identification with the other, and before language restores to it, in the universal, its function as subject." (Lacan 1953-54, 94)

In other words, the wakeful self begins to construct its identity in response to its reflection, i.e. to one-self as the other - an abstract visual signature spatio-temporally locked in the reflective axis. The early human capacity for reflective processing could mean even at the absence of mirrors as objects, that self-constructing begins with a sort of solipsistic communication. The connotations of such a proposition beckon a number of questions that bring us back to the mirror. Remarkably, the mirror, one of the oldest technological inventions, began to be commercially produced at the onset of the Scientific Revolution and did not become a common household object until the Industrial Revolution set mass production into full motion. Combined with another ancient invention, the lens, it was an integral part of two key technological breakthroughs - the telescope and the movie camera. Did the penetration of reflective objects into both everyday life and into the symbolic fields of production (arts and sciences) intensify the flourishing of Cartesian reflection in the West? Whatever the answer (or lack thereof) to this sweeping question, the cognitive role of reflection remains integral to the wakeful apprehension of phenomena. Conversely, dream is a broken mirror of the reflective process (reminiscent of the ancient mirror-as-window discussed earlier) - the images generated by the dreamer have no reflective surface to sustain and bounce them back so they morph and flow in a collapsed space-time where the dreamer and the dream coincide. In other words, the cognitive process in dream, the apprehension of the dreamed, happens directly since dream language is not signifying the way symbolic language is. A dream form can mean a range of possible things specific to the dreamer in that particular dream and may or may not have any correspondence to the wakeful assignment of meaning to a particular vision. Dream meaning emerges spontaneously in the dream and may never repeat itself. A shoe may provoke deep 
feelings of impending doom in one dream, indifference in another, and mean nothing that can be consciously decoded in yet a third one. A house is often a known location in a dream yet it doesn't correspond in the least to the actual location it represents. Dream forms are neither symbolically consistent like language, nor perceptually consistent like physical form. They have a linguistic and formal arbitrariness - the visual form is no longer a perceptual or linguistic interpretation, it is only a way of focusing the attention of the dreamer to mobilize his or her own knowing.

But how does the oneiric apparition, and its apprehension, relate to cinema? While the production process of traditional art forms is grounded in metonymic corporeal reference, cinema has no corporeal grounding. Its only contiguity is to the phantom character of the oneiric body. It could be argued that all art forms, i.e. the forms of poetic language, channel the oneiric experience into wakefulness, making an artwork more than a mere illustration of a concept. Commonly referred to as a mystifying "je ne sais quoi" in art, pinned by Roland Barthes as the third meaning that "intellection cannot quite absorb, a meaning persistent and fugitive, apparent and evasive," (Barthes 1970,44) this oneiric significance, unbound to linguistic rationalization is the life-blood, the agglutinating force, of the poetic. Barthes identifies it as the region of film language itself:

"The filmic... lies precisely here, in that region where articulated language is no longer more than approximative and where another language begins ..." (Barthes, 1970, 65)

Thus cinema not only uses that language, as an art form, it is based on it. Even the most continuous film cannot evade the structural discontinuity of montage techniques and camera movement. When a film tries to evade editing, it becomes necessarily bound to the camera position that automatically evokes the omnipresent uninterrupted oneiric point-of-view, as in the long travelling shots of Miclos Jancso or, even more explicitly, in Alexander Sokhurov's Russian Ark (2002) where the entire 96 minute long film is composed of an uninterrupted sequence shot, 
evoking the dreamy point of view of the dead narrator. The only type of cinema that manages to avoid the oneiric element almost entirely is pornography, with its insistence on raw durational footage that more often than not can do without both camera movement and editing. The viewer is glued to a permanent point-of-view reminiscent of the limited angle of the peep hole the voyeur is bound to.

Since the oneiric body is vague, fluid and polymorphic, it cannot be fragmented the way the wakeful body can. Moreover, an oneiric identity, if it could be called an identity, is always already fragmented in that it always coincides with a unique oneiric moment, i.e. there is no fixed identity that presides over dreams or agglutinates oneiric reality, yet that fluid point-of-view is consistently presiding over the dream as a singular point of awareness. The oneiric experience as destabilizing the symmetry of reflection conflicts with the wakeful technologies of the self that work to forge the symmetrical certainty of the reflective axis. The objectifying identity-casting of Foucault's discourse of power (Foucault 1990 [1976]) is implicitly challenged by the evasive parameters of poetic language. Cinema as the best candidate for the embodiment of the ex-centric locus of this language can be particularly disturbing to the wakeful dynamic of power. It can potentially disrupt not only the continuity of discursive construction of identities and intersubjective pathways (as all art forms do), but also the continuity of the sensible rendition of power narratives. In short, the intensification of the oneiric experience has the potential to disrupt the intensification of social bodies, with all the dangers and possibilities this may entail.

Respectively, there is no art form as strongly institutionalized and socially monitored (both formally and informally) as the art of the moving image. Its technological bondage has facilitated the institutionalization of its production, and more importantly, of its dissemination. Moreover, it has allowed the forging of continuities of dominant narratives with the controlled use of oneiric discontinuity - the hypnotic effect of a thus dreamified reality (perceptual 
experience imbued with the signature ways of direct apprehension) can be a powerful tool of conviction when woven into a carefully crafted dominant narrative. However, cinema is a type of assembling phenomena that invokes oneiric experience into wakefulness which thus can be subversive and disruptive to traditional strategies. This disruptiveness has been used strategically to introduce points of energy relief in the power grid and thus maintain it, but if it seeps through a hypothetical crack in that grid, it can destabilize its very integrity.

Although the advent of new lightweight moving image technologies has partially deinstitutionalized cinema, the mediumatic power grid tucks the oneiric into niches of cultural production that are marginalized, according to Bourdieu's cultural production field scheme (Bourdieu 1993), proportionately to their interest in generating oneiric language constructs. Experimental cinema is thus currently perhaps the most isolated laboratory of cultural production, not so much in terms of its institutional support, but more significantly, in terms of dissemination and viewership. The semantics generated by the technologies of the self obscure the possible readings of cinema poetics to a point of general unintelligibility, confining experimental cinema to the boundaries of the laboratory, where it produces cultural capital to be interpreted by professionals and incrementally/strategically introduced into popular narratives.

Indeed, experimental cinema becomes the bona fide apparition of a fleeting memory that does not have to be remembered - first and foremost because, like all cinema, it is recorded and can be replayed potentially ad infinitum. The oneiric is known without being familiar, but it can also be made familiar in the cinematic loop and this familiarity can then be re-established indefinitely into the unfolding of dominant discourse. Cinema captures temporal apparitions - a bona fide mnemonic device - but also potentially perpetuating those apparitions into infinite replay, which denies them historical succession, eventuating a Nietzscheanian collapse of historicity into horizonless linearity, (the straight line liberated from its only non-technological 
relation to time). This could be said for all records, ancient and modern - in a way it could be said that the record itself held the danger for Nietzscheanian temporal hermeneutics from the onset, but was contained within the horizons of the pre-modern grid of institutional power. The shift to discursive power allowed for the disappearance of the horizon and the spill of linearity into infinitude. In this flattening of the curvature of time to infinity, death itself recedes with the horizon. The oneiric in cinema however disrupts this linearity in a paradoxical coincidence with it - one can replay the discontinuous "dream" of an experimental film ad infinitum and yes, on one hand, the loop can familiarize the viewer with the apparition, but on the other, the apparition can disrupt the structural integrity of the loop, calling into question its very claim to corporeal reality. So how then can cinema, the ever-projected disembodied light, the symbolic form that most completely gathers the modernist project, die?

Recently I read on the wall of a public bathroom an anonymous graffiti: "Life is the new death!" Thinking of cinema as the modern version of disembodied life made this statement shine forth. 


\section{Chapter 7. Poetry and Pornography}

Cinema as a mirror reflects both mental and empirical movements - the movements of mind and life reduced to the mechanical movement of the cinematic machine. The physical tangibility of new technologies arrives coupled with symbolic distancing, making the Cartesian divide viewable. As a new technology, an independent apparatus that foregrounds its own way, cinema synthesizes the mimesis of empirical reality and rational (linear) thought as ways of knowing through conceptual language. It captures and records spatio-temporal events not only in shared reality, but most recently, in subjective experience as signalled in brain activity.

A study conducted by a team at the University of Berkeley decoded dynamic brain activity through $\mathrm{fMRI}$ machines that captured and recorded the signals of the visual imaging brain process interpreting moving images. (Nishimoto, Vu, Naselaris, Benjamini, Yu and Gallant 2011) The video viewed by the subjects of the experiment and the (decoded) movie of the brain, although only roughly approximate in detail and nuance, are remarkably similar in general outline

and movement, i.e. in spatio-temporal tracking. (https://www.youtube.com/watch?v=nsjDnYxJ0bo) This means that the potential for high-fidelity decoding of spatio-temporal brain imaging activity is perhaps a matter of course.

In addition to the descriptive/recording capacities of cinema, it also allows for an unprecedented sensible realization of the thinkable. Animation techniques and special effects, from a primitive optical motion reversal or speed control to the most advanced matte painting and 3-D simulation, coupled with the syntactic capacities of film editing, make the sensory rendition of any spatio-temporal thought possible. Cinema unleashes conceptual thought, allowing it to explore the geography of reason to the very edge of thinking - to the place where poetry dwells. 
Thus cinema actualizes the gift of symbolic distancing - the shift of focus and perspective. The history of the camera technology is the history of perspective, from static to zoom lens, from light to thermal optics, from heavy to light mobile technology. The camera can take us to the farthest recesses of the unseen - to open space and to the inside body - shattering our very physical solidity and uncovering all the secret spaces of our embodied experience. Through the movie camera, our temporal eye conquers deadly space without threatening our wellbeing - we can experience events beyond Earth's atmosphere and life within our own body from the safety of our technologically shielded immobility.

From the first intrusion in the privacy of the body - the public exposure of intimacy in the pornographic image - through the penetration of the flesh in $\mathrm{x}$ ray photography to the recent technologies of endoscopy that revealed our dark mysterious entrails that, not long ago, couldn't be witnessed without cutting open the flesh. And while the static camera covers this range, it is only through the moving endoscopic image, for instance, that we can witness the movement of life inside the body. The new media technologies made possible the penetration of the most secret aspects of the body - those that were shielded by the protective covers of taboo and death. In the beginning of the twentieth century we could, for the first time, witness a private lovemaking session without having to peep through the keyhole. Today we can witness the beating of a human heart without having to cut open a human body. The camera has thus allowed us to experience sex beyond Eros and life beyond Thanatos. We have come a long way from the pornographic frescoes of Pompeii or the heart-wrenching human sacrifices at Chichen Itza.

Cinematic technology as a way of spatializing space and temporalizing time, evacuating them of any (warping) presence - light shapes living on beyond life - unfolds to uncover and lay bare the conceptual expanse, in accord with Heidegger's view of the danger of technology: 
"The essential unfolding of technology threatens revealing, threatens it with the possibility that all revealing will be consumed in ordering and that everything will present itself only in the unconcealment of standing reserve. Human activity can never directly counter this danger.” (Heidegger 1977 [1954], 339)

Human activity alone, the insertion of the human body behind or in front of the camera does not affect this movement of technology, it is rather swept by it. Indeed, the hybridizing intercourse with technology is part of the inventory of technological ordering. The history of cinema and the body is launched by early pornography and, interestingly, the language of cinema maps out all the camera positions that have historically allowed for the objective observation of the body - from the fixed camera of the voyeur to the endoscopic sex act - pornography has both distanced our regard from the sexual act and, in this distancing, has brought us inside our bodies, augmenting the unseen into an impossible hyper-sensuality. Pornography's fidelity to the documentary language of cinema as distancing, descriptive and uncovering has crossed into a hyper-descriptive stand-in for reality.

Balzac famously said that "the mission of art is not to copy nature, but to express it." If "express," as the etymology suggests, means to externalize pressure, to give voice and image to the invisible and inaudible that presses to come out, it therefore refers to the domain of spatial delineation, of inside-out, which is to say that it can lend itself easily to conceptual reduction whereby the uncovering of the nature of the bodily pressures by the endoscopic camera, its deathdefying crossing of the boundaries of the body and witnessing the natural pressures that constitute it, could be reasonably argued as an artistic achievement. However, art happens as the movement of poetic language edges outside of reason and leaps (rather than follows) into the unknown, revealing the silences behind the visible, not just uncovering the unseen. Poetry, like conceptual language, can survey the land of thought but, unlike it, poetry is all inclusive, it allows 
everything, it can face anything, from the most magnificent sight to the most abominable action, not because it aestheticizes things but because it goes to the edge of things directly - it doesn't need a reason to do so.

"The arts were not derived from the artistic. Artworks were not enjoyed aesthetically...

[Art] was a revealing that brought forth and made present, and therefore belonged within poiesis.” (Heidegger 1977 [1954], 339)

Cinema actualizes the poetic edge of thought as both lived and witnessed experience, as both enacted and imagined (unlike theatre which is limited in enacting the imagined if it doesn't lend itself to human embodiment). This unique capacity of cinema - to make present the poetry of thought and apparent to perception - grounds its authenticity in the artistic value of a film work:

"A film is never really good unless the camera is an eye in the head of a poet. Distributors, naturally, are all of the opinion that poets don't sell seats. They do not discern whence comes the very language of the cinema. Without poets, the vocabulary of the film would be far too limited ever to make a true appeal to the public." (Welles 1958, 164)

Thus the symbol as the signature of language reveals its gift as twofold - distancing (conceptual) and leaping (poetic). It is the poetic gift of the symbol that saves cinema from banality, from being a mere instrument of conceptual thought, a scientific tool of "unconcealment" that lays bare things as "standing reserve," of sensationalism at best:

"If the cinema had never been fashioned by poetry, it would have remained no more than a mechanical curiosity, occasionally on view like a stuffed whale.” (Ibid.)

Moreover, it is poetry that saves humanity from the threatening independence of the camera eye: 
"Everything that lives... derives from the ability of the camera to see. It does not see naturally in place of an artist, it sees with him. The camera at such instants is far more than a registering apparatus; it is a means by which come to us messages from the other world and which let us into the great secret. This is the beginning of magic. But the charm cannot work unless the eye of the camera also is human. That eye should be on the scale of the human eye. [..]

A film is a ribbon of dreams." (Ibid.)

When Atum ejaculated the world into existence as s/he touched her (male) penis with his (female) hand and Ptah dreamt creation in his heart, speaking the world into existence, the world was begotten by both divine semen and divine word. It was conceived in the carnal state of intercourse between male (phallus) and female (hand) as well as in the mental state of dreaming the two ends of conception that would eventually become the basis of Cartesian duality. techne. [...]

"There was a time when it was not technology alone that bore the name

There was a time when the bringing forth of the true into the beautiful was called techne. The poiesis of the fine arts was also called techne.

...the arts soared to the supreme heights of the revealing granted them. They illuminated the presence of the gods and the dialogue of divine and human destining. And art was called simply techne. It was a single, manifold revealing." (Heidegger 1977 [1954], 316)

The "manifold revealing" of the hidden and remote in the very temple of the unknown is not mimetic. Techne as a tool of truth, and not a system of control, is a techne grounded not into the conceptual, as modern technology would claim, but in the poetic. The poetic way to truth is a direct vision in the domain of the gods, a disruption of continuity, a leap of sorts, such as the begetting power of the sexual intercourse and the otherworldly visions of dreaming. (Sexual intercourse as poetic in its capacity to bring forth another life body from a form as dissimilar to a mature human as the cohesion of two biological cells (Socratic natural poiesis). Dreaming as poietic in its capacity to cohere form in ways that defy spatio-temporality.)

Departing from Heidegger's view of modern technologies as challenging to and extracting of nature (Heidegger 1977 [1954], 327-328), we arrived at a view of the media 
generated by the Industrial Revolution as abstracting. This abstracting capacity of modern media technology accounts for the effects of Benjamin's mechanical reproduction (Benjamin 1939). Abstracting makes possible the industrial re-production - form free from the spatio-temporal limitations of its original embodiment, and multiplied potentially ad infinitum. Its original embodiment is replaced by its technological encasement. Technological media encasement is the dwelling of original disembodiment. It supports and promotes the duplication and re-play of disembodied form, it has no functional relationship to the specificity of that form, meaning that the same film projector promotes the re-play of any film just as a film camera can record a limitless number of different film scenes, regardless of their content. When I reach out to open a book, this gesture is embedded in the spatio-temporal parameters of my physicality - I need my hand in order to carry out the action of opening a book - a gesture that can never be rewound or duplicated, but can only be closely imitated in another unique moment as in Heraclitus's words, "no man steps in the same river twice."

Furthermore, if the page I opened shows a Necker cube, the camera can render the 2-D image, but cannot render the shift of my mental focus that plays with the direction of the cube in depth. However, through careful management of angle and movement, the filmmaker could perhaps construct a sequence that mimics that shift of mental focus and renders it visible. This sequence would bear witness to the linear nature of the perceptual complex concurrent to empirical reality - one cannot simultaneously perceive the two directions of the Hecker cube in depth, just as one cannot simultaneously stand to the left and to the right of a physical cube. While the image on the printed page would mobilize all those perceptual experiences, the film sequence will copy the image and imitate the process of seeing it in a directed passive-viewer demonstration. 
In other words, original embodiment and technological encasement are defined by their functional relationship to form - while original embodiment coincides with and reveals its form, industrial technological encasement promotes formatting. Unlike the traditional technology of painting for instance, where the painted image coincides with the paint and the canvas (revealing the tools and techniques of rendering the image), new technological encasement both suggests and conceals its original tools (the movie camera, the film stock, etc.) in order to foreground an abstracted form that does not belong to its original body complex. That abstracted form in cinema is a moving image (the sound of cinema exists as an independent technology). It is a reflected body, a record of a mirror image or, put otherwise, a symbolic language built of reflections, not of symbols (confabulated forms that can, but most typically, as in the case of symbols par excellence such as words and letters, do not bare any similarity with the original form they refer to). The built-in reflectivity of film language makes it lightweight, descriptive and enclosing. Yet language is symbolic, indeed it is the beginning of symbolic being, and therefore it best unfolds through the manifold of symbol, not the flatness of reflection.

The marriage of photography and movement in cinema made reproduction an integral part of cinematic representation. Regardless of its technique, the final film print can be copied and distributed many times, each copy containing the exact same set/sequence of images as the original capture. The re-play is a hi-fidelity copy of the original and can take place simultaneously at different places - industrial media allow for a ubiquity of form unique to new technologies. This is a radical departure from the traditional re-play (theatre, camera obscura, concert) always originally embodied, i.e. performed, therefore spatially bound, and subtly singular. However, recording is not yet a departure from the pre-industrial technologies of metal casting and printing, which Benjamin groups together with the Industrial technologies of photography and film as means of mechanical reproduction (Benjamin, 1939). The split between 
these two modes of media production, the pre-industrial and the industrial, becomes apparent in their relationship to the original. Pre-industrial technologies are distributors of an original artifact that cannot be falsified since its originality is in the reproducible form itself (e.g. written word or cast metal sculpture). The mold of a bronze sculpture is not the work of art, the cast bronze is. Even if a shape is directly cast from the world of objects, such as a tree branch for example, its metal version is a radically new type of embodiment that, by virtue of its materiality, adds a new interpretive layer to embodiment (thus precluding full abstraction). This said, cast metal became one of the core technologies of industrial production. As a communication/art medium however, it remained closer to traditional technologies. As an Industrial technology, it remained at the first degree of mediumization (of constructing encasement) - the production of machine components. This displacement of metal casting from art to industry, from object to component, exemplifies the way Industrial production pushed embodiment below a new mediumatic layer, the layer of audio-visual frequencies (light and sound) which would be henceforth the isolated level on which meaning would be consolidated and processed. In other words, pre-industrial technologies were only precursors of the high fidelity abstraction that became possible with photography and its derivative media technologies of the moving image.

The photographed cinematic event re-presents the original embodied event as a document. The interpretive aspects of a document are format-related, compositional and contextual. The painted image inevitably bears a personal interpretive imprint on every layer of the work while the photographer's presence resonates only in the photograph's choice of scene (that which marks the beginning of the painter's work). In addition, the photographed image foregrounds the documented event over the craft of the photographer. In fact, the camera can be set in such a way that the photographer is entirely replaceable or disposable from the production process. Thus the industrial/photographic image becomes contingent on absences - both that of 
the event itself and the possibility of the absence of the photographer. In short, the two aspects of re-presentation that photography brings forth are the new temporal mode (re-playability of reproductions) and the fidelity of the documented image to the original.

In cinema the fidelity of the documented image is temporally enhanced with the introduction of the realistic 24 fps projection rate or as Walter Benjamin (Benjamin 1939, Ch. 1) roughly assigns it to the capacity of moving image cameras to capture the actors at their talking speed (even in silent cinema). This temporal abstraction is not only unprecedented, it allows new media technologies to reveal a mode of disembodied being that concurs with the very concept of reality - it promises a full realization of mimesis, akin to Jorge Louis Borges's perfect map overlapping physical reality precisely in scale 1:1 (Borges 1975), and one that is also re-playable potentially ad infinitum. A type of mimesis that allows for the colonization of time. Benjamin's vision of film's social significance as a cathartic "liquidation of the traditional value of the cultural heritage" (Benjamin 1939, Ch. 2) stops short of recognizing a much more radical significance of film's mimetic temporality - that of obfuscating physical temporality itself, the experience of irreversibility, entropy, decay, or to use Heideggerian language, of being-towarddeath. Cinema as life beyond death - cinematic temporality as para-mortality.

With the advent of new (media) technologies there is a reversal of Platonic mimesis - the world of ideas is extracted from empirical reality (considered by Plato mimetic to ideas) and rendered "ideally" present in the photographic negative film. With the addition of sound, it begins to open up the avenues of full sensory engulfment, or full reality stand-in, toward the eventual augmentation of that reality. The explosion of the prison of embodiment in cinema (Benjamin) makes possible travel without travelling, connecting without contact - it makes possible an augmentation of our world that is not merely gratuitous, but also miraculous. If mirror neurons allowed us to feel our bodies visually in places we could never optically access, and the silver 
mirror allowed us to actually see those parts of our bodies, cinema allowed us to see versions of human embodiment in the world that we could not spatially access, surpassing our local knowledge of ourselves and expanding it into a knowledge of humanity. We were no longer entities using technology to know ourselves but we become techno-cognitive extensions, tentacles, a technique of humanity to know itself. Moreover, a technique of the Earth, as our host organism, to know itself. Indeed, with satellite photography, we extend this cognitive scanning to open space. Knowledge as travelling, sharing and incorporating back the condition of the world that is furthest from ours is the way the mimetic power of cinema cultivates a world-body. It also underlines the growing power of documentary filmmaking as an authentic form, true to the cinematic medium.

The mimetic power of cinema, as magnificent as it is, is still an audio-visual approximation of reality. A coffee cup on screen remains an object to be witnessed, not held. However, a future where technology can decode and then reversely induce the range of sensations aligned with holding a coffee cup is not far away. From advances in holographic fidelity to tactile devices replicating the sensory experience of spacial interaction with the projection, the mimetic potential of cinematic technologies promise and threaten to colonize the entire sensory horizon. Video imaging of brain activity is the precursor of a whole different type of media layering, one that completely forgoes formal abstraction and symbolic language. A medium that interacts directly with the brain and thus produces sensations and thoughts that appear native to the subject has no use for poetry or even (syntactic) language. Indeed, it has no use of distance as the gift of concept - the gift that allows us to roam freely the territory of form. When technology uses distance to erase it, when it bypasses the space that conceptual thinking opens to allow a free negotiation between its components, and when it invades the no-space of dream and though, its dangers cross over from the territory of thinking to that of perceiving. How would we make the 
difference between original and mimetic experience once this potential of new media technology is fulfilled? Would our body be tricked to believe it has drunk the coffee, eaten the food when it hadn't? Would that mean the discovery of new corporeal frontiers where mimesis produces reality and we turn out nurtured and satiated by signal only, or would that simply mean a new way of dying - death by delusion?

The saving power of mimetic cinema is precisely in its incompleteness which imbues an image with meaning (by dwelling in the house of thought where a disembodied shape can be sheltered and nurtured). Thus the cinematic rendition of the experience with the Hecker cube, as close as it could be in optically emulating the experience of visual perception as a shift of mental focus, and as fidel it may be to the spatializing linearity of sensory experience coordinated by mental processes, it does not belong to thinking. In fact, it is alien to thinking, not only because, by offering its description of the process of viewing the cube, it actually pacifies the viewer and inhibits the very mental process it promotes. Moreover, it does not and cannot transliterate the characteristic most intimate to thought - non-spatial simultaneity (most clearly apparent in dream). Somewhere in the manifold of thought, somewhere that is neither the 2-d flattening that explains the cube's rationale to the eye, nor in the perceptual field, we hold the image-knowledge of that simultaneity of the Hecker cube's dual perspective. It shimmers in the back of thought, where dream images are stored. It is from that dream inventory that the possibilities of viewing and seeing the Hecker cube unfold, and it is to that dream realm they allude to. In the mystery of its simultaneity - a mystery we envision and know yet cannot experience sensibly - the cube becomes meaningful.

Until the development of cinema, one aspect of image remained consistently elusive: the disembodied moving image remained the exclusive domain of dreaming experience. Until cinema, all experience of the moving image outside of dreaming was necessarily originally 
embodied. Cinema externalized this most private space/time-the oneiric space-time - rendering it open to the public eye. It extracted the mental vision from the sensory isolation of dreams and rendered it accessible to the senses. Thus cinema made possible two hitherto impossible experiences: the sensory interpersonal viewing of an oneiric-type vision and the repeated viewing of the (documented) disembodied moving image, the event. This disembowelment of the moving image afforded a clinical recording and exposition of spaces that would be otherwise visually unapproachable or unreachable, i.e. untouchable either by virtue of physical or social limitations. In other words, it afforded an unfolding of the Merleau-Pontean visible (Merleau-Ponty 1968) into unconcealment; all those visible yet hidden surfaces were made optically available. Thus, the forbidden and the hidden of the body were unconcealed, from pornography to endoscopy. The mobility of cinematic technology would take the camera eye to the macro perspective of outer space making the unreachable see-able, while the manageability of direct-to-medium techniques would produce the first nano-movie, A Boy and his Atom (2012, IBM) making the un-see-able viewable.

But what would all this disembodiment mean to the individual viewer and his relationship to his or her own immanence? Where cinema serves as a technological tool of scientific mapping, such as in medicine, space exploration or nano research, it has little relationship to the subject, to the reflective point-of-view. Documentary filmmaking, as far as it bridges the explorative aspect of cinematic documentation to audience, remains rooted in the scientific imperative of cinema, driven by a cognitive view that is primarily informational and communicational.

However, in the area of film as a description of the embodied human condition, pornography occupies a unique place - it uses the descriptive powers of cinema to bring to public attention a hitherto private act, in a way neighbouring the externalization of dream. But, unlike 
oneiric film where dream is made explicit by allusion, by poetic insinuation, sexual intimacy is simply uncovered, undressed, made naked by documentation. Pornography did not begin with cinema - pornography was fulfilled by cinema, cinema became a condition to the fulfilment of pornography.

Although etymologically the word pornography means "description of a prostitute," through time it has acquired a more general meaning referring to the full range of explicit descriptions related to the sexual act. With techno-scientific precision, cinematic documentation severs the relationship of the sexual act to natural poiesis and the poetics of the flesh, and, through its presentation to the audience, mobilizes animalist mirror neuronal activity, basic physical communication that isolates the mechanism of sexuality to near perfect biotechnological perfection. This isolation of sexuality disturbs its socio-cultural contextualization and forges its problematic cultural position. Pornography as description both forges its bond with cinema on one hand, and destabilizes its relationship to poetics on the other. It renders the originally poetic starkly technological, it severs techne and poiesis in a way only possible through the highly conceptual, separating and distancing tools of industrial technologies. This displacement, and not moral coding, is what disturbs social attitudes to pornography. It intimates the mechanizing dangers of the techno-scientific cognitive formatting, potentially threatening to colonize the vital spaces of the body.

The success of cinematic pornography is due to exclusive use of the photo-timed capacity of true-to-life morphological reflection. Its descriptive commitment has no use for the syntactic capacity of time-space manipulation that is the poetic signature of cinema. This syntactic flexibility pertains to cinematic language regardless of whether the image has been extracted (documentary) or hand-made (animation). By contrast, the morphological reflection as imagebound is contingent on the medium's capacity to extract/record visual information. In other words 
from the two ends of cinema they occupy, the externalization of the dream and the publication of the sexual act correspond to the two signatures of cinema language, the poetic manipulation of visualized time and the documentary extraction of the image. They stand at the two ends of the cinematic destination as language - poetry and reason as the two ways of language.

The high-fidelity image captured at a range of speed that largely surpasses the natural parameters of time perception defines the morphological temporal realism/continuity of a single shot. Conversely, the mobility of the (disembodied) image allows the manipulation of temporal continuity in editing. In other words, while the fidelity of the image and its movement defines its realistic duration on screen, the fact that that image is disembodied affords the manipulation of the duration (both in terms of speed and direction as well as in terms of relationships to other images), and therefore accounts for the (aesthetically necessary) break from the rules of realism in film time construction. (1)

How did the gesture of masturbation turn from a poetic metaphor of subject of pornography? Although the descriptive isolation of the sexual act appears to be the minimum condition for pornography, the fulfilment of pornography seems to be contingent on the emergence of photography. While the emergence of pornography destabilizes the cultural pregnancy of the sexual paradigm (by subverting the sexual act as a cultural idiologem), the cinematic fulfilment of pornography stabilizes abstract(ed) sensuality. The pornographic still picture is a poster of the pornographic event that would realize itself in film. It was the cinematic capacity to capture realistic time movement that allowed pornography to unfold as pornography (and fully disengage from erotica). The temporal realism of the documented sexual act was contingent not only on cinema's photographic capacity to extract the image but also on its capacity to capture the sexual act's uninterrupted rhythm, its capacity for documentary time. Through rhythm, the visualization of the tactile became not only more actual but more akin to the 
mental processing of sensual reality. Cinema brought (visual) mimesis to a whole new level. Cinema brought forth a form of ideation of embodied reality in its mirrored disembodiment that had a twofold effect - on one hand it released phenomena from the limitations of embodiment which allowed them to behave as ideas rather than bodies; on the other hand, its mirror effect engaged the senses in neurological ways that evoked ever so closely embodied experience. The formal kinship of the cinematic experience with embodied experience has a number of ramifications on the understanding of reality - on one end of the spectrum is Catherine MacKinnon's equation of the pornographic document with an event that is equivalent, or at least functionally equivalent, to the embodied sexual act (MacKinnon 1993). On the other end is the postmodern vision of embodied reality as an ever receding expanse of textual signs, subject to an ever relational interpretation. Cinematic pornography's engagement with visceral reality affords stimulation akin to MacKinnon's event - a sort of realized fantasy that brings, among other things, solitary masturbation out of its mental short-circuit. The fantasy that is played out in masturbation is brought out into the outside where the pornographic film can be a kind of another who has been invited into the masturbator's space (affording sensory stimulation without the intrusiveness of physical voyeurism). Pornography brings solitary masturbation closer to shared reality by externalizing the fantasy, i.e. offering a fantasy enactment as a documented event. On one end pornography extracts the (moving) image out of embodied reality and on the other, it plays it out as an extracted mental process (the pre-technological moving image, the dream) - an unprecedented way of converging mental and physical reality that was only made possible by cinema.

The convergence of the mythically separate creative acts of Atum (the original masturbator) and Ptah (the original dreamer) into one, mutually contaminating, event - the pornographic film - happens when a clinical eye, such as the film lens, captures a messy physical 
act and transplants it into a host as far removed from the carnal as the light of film projection can get. Unlike medical capturing, the clinical distancing is not maintained in the viewing - the viewing of the pornographic does not retain the clinical distance of the lens, but uses it to gain proximity to the captured event. This transplantation of a Dionysian chthonic experience into the Apollonian (solar) domain turns dualistic intuitions on their head. What makes the sexual act pornographic is not simply the rendering of a private act public, the breaking of moral taboos or the mechanization of sex - it is in the libidinal (in the Lyotaridan sense), contamination of one domain with elements of another. When the representation of the sexual act becomes itself an act (that affects bodies directly, e.g. erects penises) the contamination is libidinal (it transfers energy from one domain to another). This libidinal transfer is made possible by the sanitation of the cinematic moving image which removes the threat of direct contact through touch, thus removing the threat of violation and infection. Once removed, fear and disgust can become part of the erotic play, especially if that play is decontextualized and semantically disconnected. There is an overarching disgust with the very phenomenon of pornography that seems rooted in contamination, rather than in any idiosyncratic or social predispositions of physical likes and dislikes. That general disgust is an emotional reaction to the solar/chthonic contamination, long before it becomes disgust with the proximity of the viewer to a sexual act as a broken taboo. That overarching disgust is extended to the experience of the sexual act as a sanitized, therefore physically inconsequential, event. The capacity of pornography to overwrite fear and apprehension (of physical proximity) through the distancing of cinema while affecting bodies directly by bringing the image close demonstrates the power of cinematic technologies to tame physical reality and overpower bodies in doing so. The distancing and nearing effect of cinema, exemplified by the aerial view and the close up, forge new type of hyper-real proximities. This 
new type of distancing mobilizes the cognitive aspect of the pleasure mechanism, as theorized in Benjamin's One-Way Street:

"For Benjamin, the experience of pleasure is predicated upon distance. Although this pleasure is not simply located in distance itself, it does reside in the possibilities that distance opens up. That is to say, in order for distance to be traversed or, as Benjamin says, "conquered," distance first must exist. There can be no conquering or transgression without the perpetual impetus of something to be conquered or transgressed." (Richter 2007, 57)

Future pornography can realize the radical potential of the descriptive power of cinema in a new type of immersive technologies that mobilize the entire sensorium and actualize McKinnon's pornography as act. With the decoding and mechanization of key aspects of the Ego Tunnel, future media can replace entire bands of reality that cinema had only intimated. A fully interactive 3-D holographic model of a human could bring together three industries pornography, sex toys and sex work - into one comprehensive product that completely replaces all human presence those industries were developed to compensate. This potential of technologized pornography, made explicit by cinema, is revealing of the dangers and opportunities of new media and the direction techno-science is taking us. The radical alienation and respective deactivation of the embodied human condition intimated by cinematic technologies is a danger much more clearly demonstrable than the openings affordable through those technologies. Because, for those openings to work as openings, they must lead beyond the solipsistic mirror world new technologies enforce, i.e. beyond the language and mind-set promoted by those technologies and dictating our thinking of them.

Cinematic distancing also affords a manipulation of the moving image that can impose any imaginable version of embodied reality (from defying gravity to reversing and replaying time) into the solar domain of cinematic projection. However, unlike the descriptive transfer of pornography, film montage and image manipulation belongs to a language native to the domain 
of the disembodied moving image - the domain of thought. This is why montage, like the entire complex of moving image manipulation from in-frame through frame-by-frame to shot-by-shot, is not a contaminating visual transfer - its tools use that which is true to language, its deceptive power as poetry. When openly using the poetic as thought, the mental mimesis of cinema is no longer a mimesis, but a way of being with cinema, a way of channelling thought by actualizing it in the interplay with sensible reality. Such is the intervention of the arts.

Montage fulfils the cinematic, it is the structure of cinema as the intersection of dreamthought and technology. Montage is drawn from the language of dream, the language of the imagined unhindered not only by physical but also by linguistic constraints. However, media as technological extensions of language realize dream's poetry, and dream as way of poetry, through language. The oneiric character of montage, the poetic in film, is on its native scene, the solar scene of Apollo, while pornography remains ob-scene as the extraction of the carnal/chthonic and its transplantation into the linguistic/solar. Yet, the pornographic (as a description of the sexual) is also fully realized by the high fidelity recording capacity of cinema. So, is cinema to pornography what poetry is to cinema, each pushing the other to its conclusion that can both stifle or liberate it? The hypnotic dangers of thought mimesis are just as potent as the dangers of corporeal displacement in reality transference. And yet pornography is fulfilled by cinema in a way similar to the way cinema is fulfilled (and not fulfilling to) by poetic language. Poetry and pornography meet in the cinematic body in a way similar and yet entirely dissimilar to the way Apollonian inspiration meets chthonic fumes in the body of the Delphi Sybille.

About six thousand years after Atum ejaculated the world from his/her phallus and Ptah spoke it into existence, could pornography be seen as the speaking phallus and montage as the ejaculating mouth? If so, what would that mean to the understanding of both cinema and the embodied reality it confronts? A way to bring the two together through the reconciling power of 
art could be a way to exit their language by pushing it to its edge. Could a fusion of the chthonic energy of pornography with the Apollonian dream of montage be a way to both imbue the medium with a life of its own (true to the technological imperative to independence) and at the same time open it as a poetic space (true to the linguistic commitment to knowledge), as a way to the edge of the known?

We are thrown into a world that we begin to assemble through the experience of embodiment. It is a wonderful world of possibilities and limitations - once we master the possibilities, the limitations start blocking the horizon like a prison of thingness, the prison of being. In the tyranny of experience language comes to the rescue, extracting us from the immanence of being and flying us around on the wings of abstraction. We escape experience through language, merging our experience with experiences of otherness, with the experience of language. But the tyranny of language is liberty - the tyranny of infinite choice. The only way out of this tyranny is to bring the experience of language back to our immanence, to choose our embodiment, our original limitation as the only possible choice, the only way to freedom. Cinema opens this possibility with the threat and the promise to bring us to the edge of language on the wings of the photon. Whether it would save or annihilate us is a matter of distancing. The aerial view of a god's perspective is but the distance new technologies afford. And the safe way to look at them requires distance. Like the sun that projects its light to nourish us, the nuclear explosion of cinema brings back to us its finds - fragments of that explosion that, through the magnifying optics of the close-up, can take us to the end of life itself. Cinema is the prophesy of solar death.

\section{NOTES TO CHAPTER 7:}

1. This understanding of film duration, film image, film movement and film time differs (although does not exclude) Deleuze's movement-image (Deleuze 1986) by which he roughly refers to the shot as defined by the movements of images and his time-image (Deleuze 1989) by which he refers to interrupted 
temporal linearity such as the temporality of memory. Although Deleuze's consideration of mnemonic temporality (as mental temporality) is akin to my consideration of oneiric temporality vis a vis film, the distinction of dream as unsolicited imagery (emerging in a viewership relationship to the dreamer who is typically in core consciousness mode) is essential to the advancement and precision of my argument.

2. The neurotic attempts to establish and realize a transfer of human intelligence into the machine attests to the extent to which the technological imperative, expressed in technology-as-human-embodiment, has permeated our collective mind. 


\title{
Conclusion
}

Art or industry, medium or instrument, language or dialect? The questioning of cinema has brought us to poetic language as the syntax of the cinematic but let us remember the way of poetry as more than a language technique, as that which leads beyond the forest of consensual reality:

"...[poems] bear witness to an aspiration to cross the line, to go against the current, to rediscover the great calm lake where time rests from its flowing. And this lake is within us, like a primitive water, like the environment in which an immobile childhood continues to reside." (Bachelard 1971 [1960], 111)

The breathing gaze of Tarkovsky's camera can invoke this timelessness as much as the leap through geography of Deren's dancer, or the oneiric language of Juan Ramon Jimenez:

\author{
"I am not I. \\ I am this one \\ walking beside me whom I do not see, \\ whom at times I manage to visit, \\ and whom at other times I forget; \\ who remains calm and silent while I talk, \\ and forgives, gently, when I hate, \\ who walks where I am not, \\ who will remain standing when I die."
}

The poet intuiting a dual being beyond the trivial duality of conceptual organization, a way of being with oneself as a mystery, a being both known and unknown, both near and far. The one who does the daily gestures of consensual reality, the one who lives and dies, and the one who dreams all that, the witness, the silent viewer in the dark of the movie theatre. Juan Ramon Jimenez flies us to the night garden of timeless twofoldness where "all the senses awaken and fall into harmony in poetic reverie. Poetic reverie listens to this polyphony of the senses, and the 
poetic consciousness must record it. " (Bachelard 1971 [1960], 6) Cinema immerses us in this sensual polyphony, it has the power to make poetry flesh the way sex can make love flesh, a reunion of realities, a sort of dreaming awake akin to Bachelard's reverie:

"...in contrast to a dream a reverie cannot be recounted. To be communicated, it must be written, written with emotion and taste, being relived all the more strongly because it is being written down. Here, we are touching the realm of written love... There are still souls for whom love is the contact of two poetries, the fusion of two reveries." (Bachelard 1971 [1960], 7)

In this sensual fusion of experience and language in poetry as reverie, we encounter the cinematic. The poetic movement of cinema, its unique capacity to sensually enact the timeless nospace of poetry, can redeem the abstraction of seeing, the disembodiment of abstraction and the dismemberment of conception, can redeem the industrial imperative to the pornographic zombification of the body, into a new type of dream consciousness:

"But the world dreamer does not regard the world as an object; the aggressiveness of the penetrating look is of no concern to him. He is the contemplating subject. It then seems that the contemplated world passes through a scale of clarity when the consciousness of seeing is the consciousness of seeing big and the consciousness of seeing beautiful. Beauty works actively on the perceptible." (Bachelard 1971 [1960], 185)

It is this dreaming-as-thinking, this poetic way of seeing big, that first inspired humanity to envision the fantastic futures of technology we live in, and not the penetrating eye of reason. From a tool of the poetic vision reason has turned poetry into its tool, a tool of manipulation and propaganda, of playing out the fallacy of language in the name of a truth that is embedded in that language-as-fallacy itself. It has foregrounded its truth and has obfuscated its original drive:

"The need of reason is not inspired by the quest for truth but by the quest for meaning. And truth and meaning are not the same." (Arendt 1978 [1971], 15) 
When we see meaning as that which has been passed through the purgatory of thought, as that which pertains to the thinkable, and truth as that which is beyond the thinkable, beyond the speakable and the knowable, that which is revealed at the edge of those activities, the tools and values of science and industry (conceptual thinking and truth as correctness) return to their rightful place as means not answers:

"...thinking aims at and ends in contemplation, and contemplation is not an activity but a passivity; it is the point where mental activity comes to rest. According to traditions of Christian time, when philosophy had become the handmaiden of theology, thinking became meditation, and meditation again ended in contemplation, a kind of blessed state of the soul where the mind was no longer stretching out to know the truth but, in anticipation of a future state, received it temporarily in intuition. (Arendt 1978 [1971], 6-7)

This contemplative state of thought beyond thinking, opening onto truth beyond grasp, hovers in the region of Bachelard's reverie - a liminal state that settles in the cracks of the known:

"...we believe that one can know states which are ontologically below being and above nothingness. In these states the contradiction between being and non-being fades away." (Bachelard 1971 [1960], 111)

Dreaming as the source gate of those states is where we experience core consciousness, the point-of-view as a witness that observes the ambush of thought as otherness:

"One might wonder whether there really is a consciousness of dreams. A dream can be so strange that it seems that another subject has come to dream within us." (Bachelard 1971 [1960], 11)

In wakefulness that other one, the dreamed, takes over as the seat of awareness and the witness, the dreamer, steps aside, overwhelmed by the chatter of the inter-active mind. But back 
in dream, it is that interactivity of the mind that can augment the presence of the witness reuniting it with the witnessed. Because in dream, to stabilize a picture, one has to remember the continuity of daily life. Even when one does not remember one's wakeful identity, just remembering to follow one's movements sequentially can slow down an ordinary dream into a lucid event. The rush of oneiric thoughts then stabilizes into a scene that shimmers with contemplative duration. Durational time comes back to us as this poetry of lucid dream when we are watching a long shot in a Tarkovsky film. The moebius torus of dream is united in the cinematic experience in ways that promise augmented awareness on the condition that we are not swayed by a reversal of values and their foregrounding of augmented reality. Because doing the latter is merely putting the cart in front of the proverbial horse since cinema as an act of consciousness shares the "essentially augmentative potential of all awareness," (Bachelard 1971 [1960], 6) and therefore would inevitably lead to an augmented reality. So, the technological advancements of cinema, if they are to serve as stepping stones to an augmented reality rather than as augmented-reality traps, have to give way to poetic language, to the art of cinema, that has the potential to persistently interpolate and reunite the dreamer and the dreamed into consciousness. Cinematic technology is miraculous in the way it can stabilize a reverie and turn it into physical experience just as reason is miraculous when it invades a dream and brings duration to dream-time. In cultivating being-in-dreaming as the other side of being-aware, rationality becomes a wonder, and throws an entirely different light on Descartes "I think, therefore I am." A light that Descartes might have intuited when he reduced consciousness to thinking and postulated this reduction as the condition of being. If dream is indeed the gate of thinking, then a more precise way to rationalize being, while keeping in style with Descartes, would be to say "I dream, therefore I am." However, when this dream is reduced to a kind of thinking and thinking is reduced to reason, the latter loses its miraculous shimmer, its poetic power to interrupt realities. Instead, it 
becomes a blanket that stifles the miracle of being. In cinema reason comes back to the region of the miraculous, it promises to bring back all that has been obfuscated by the Cartesian reduction. Cinema as industrial technology radicalizes abstraction; cinema as poetic language imbues this abstraction with life.

This entire thesis about the movement of language, poetry and cinema is summed up simply:

"Poetry is one of the destinies of speech. (...) One would say that the poetic image, in its newness, opens a future to language." (Bachelard 1971 [1960], 3)

One would also say that poetic language, in its newness, opens a future to cinema as poetic image. And it is this newness that guarantees the return of the miraculous as the topsoil of reality, which, being but a shimmer, allows the reason to shine through with all its magical powers. Because when our reality is arranged the other way around and reason covers the terrain, its opaque blanket conceals the shimmer of the miraculous, banalizing everything it touches. Dreams become but fanciful shadows of the mind while poetry is employed as a mere servant of industry, a utility that helps communicate ideas, a peg in the propagating machine of technoscience.

This work has been as much a questioning of cinema as it has been a methodological intervention deliberately crafted to allow both poetry and reason to lead whenever they needed to, promoting their reunion in language as art; language as art-iculation, as a way to meander and sculpt one's way toward that which is beyond language. This is why (poetic) language at its utmost overthrows language just as knowledge is oriented toward the unknown. This is also why the proverbial rebelliousness of art is not just a youthful impulse, but a signature inherited by language itself as, in its final hour, language revolts against language in order to open up to the 
silence of the unknown. And whatever answers we may have found on our way through cinema, more important are the gaps and pauses we may have cracked open toward that which lies beyond our capacity to articulate. Because "to lose the appetite for meaning we call thinking and cease to ask unanswerable questions [is to] lose not only the ability to produce those thought-things that we call works of art but also the capacity to ask all the answerable questions upon which every civilization is founded." (Arendt 1978 [1971], 62) Before all, this study has been a quest to shine awareness on as many ways cinema may generate meaning as our own capacity for thoughtfulness, i.e. for generating meaning, allows. It has been a meditation on thinking about technology as a way of thinking.

And yet, within that meditation, like the other perspective of a Necker cube, that which is answerable remains discussable. Then, a question beckons - if Heidegger's language, the language we stayed with the longest, is the house of being, what, if anything, is cinema the house of? As an industrial technology, i.e. a medium that dismisses human embodiment, isn't it a candidate for housing non-being? Industrial technology as the house of non-being! In the way cinema intensifies engagements with both science and art, reason and poetry, technology and mind, it lays claim to reality itself. But, as an art(iculation) that brings together dream and wakefulness, cinema is a great shelter for reverie. Even the most banal cinematic documentation plunges us into a different temporality and suspends us between points-of-view and languages, between being and non-being. But cinema is a shelter at best, not a house, not yet anyway. Because beyond that which is humanly relatable in cinema, it is also, and importantly, its own medium/language - the voice of industrial technology itself threatening to outcry the voice of humanity. 
Cinema has the capacity to both intensify conscious experience and to dissolve it. The gift of cinema, that which it may eventually come to host as medium/language - a new augmented kind of embodied consciousness - is shadowed by the danger of cinema as the potential host of the zombification of life - a new stunted kind of body and consciousness. This study has insistently foregrounded the critical importance of art not as the other of science but as that which science makes part of. Which is to remind us that poetry is not only a kind of language but, more originally, language is a kind of poetry. When language loses that perspective, when it reduces itself to the limitations of its syntax, it subjects itself to an aspect of itself. The technoscientific mindset has been promoting precisely such a development of culture as language-reality - a safe, simple and fast way to perpetuate language through conceptual thinking and thus obliterate the danger of (language) mortality that is implied in the quantum leap poetry invites. This becomes explicit in cinema where technological developments occupy the forefront of cinema aesthetics, while the language of cinema not only stagnates but devolves back to basic narrative principles. The regurgitation of basic cinematic formulas, known to trigger specific reactions with an almost clinical certainty, is systematically embellished with vignettes of technological innovation. The use of technology as decoration can be as dangerous and ob-scene as the use of cinematic language for promotion. The key term is "use" - the utilitarian drive of technology coupled with the idea of purpose as meaning delineating the movement of conceptual language. The antidote to usefulness is beauty. Beauty as the truth of poetry, of language as a kind of poetry, of art as that which brings together the dreamer and the dreamed, the world and the word in an ecstatic fusion that promises to generate the energy for the quantum leap into a new awareness of a new augmented reality:

"Beauty gives relief to the contemplated world and is an elevation in the dignity of seeing at the same time. When one agrees to follow the development of the aestheticizing psychology in the double valorization of the world and of its 
dreamer, it seems that one knows a communication of two principles of vision between the beautiful object and seeing it beautiful. Then in an exaltation of the happiness of seeing the beauty of the world, the dreamer believes that, between him and the world, there is an exchange of looks, as in the double look from the loved man to the loved woman." (Bachelard 1971 [1960], 185)

Granting the reciprocity of love between the world and the dreamer, beauty redeems the interaction between the world and the point-of-view we have been famously forewarned about by Nietzsche:

"He who fights with monsters should look to it that he himself does not become a monster. And when you gaze long into an abyss the abyss also gazes into you." (Nietzsche 2003 [1886], 69)

When touched by beauty, from a dangerous, dividing and potentially contaminating, act in the binary world of opposing forces conceptual thought is immersed in, interaction becomes a way to alignment and union, a way to love, to Arendt's amori mundi. The new power that this fusion promises is not the power knowledge needs to construct a cultural stronghold (Foucault) but the power needed to prepare for a meeting with knowledge as the unknown, to open to that which lies beyond the stronghold, the power to fuse into a new tensegrity structure that can sustain that unknown as a new type of knowledge. Knowledge as freedom from the known. Somewhere in the cracks between technological fantasies turned reality and poetic reverie coming audio-visually true, between the pornographic abstraction and the poetic immanence, lies the promise of cinema to prepare us for such an unimaginable yet thinkable crysopoetic leap. If only it doesn't lull us in irreversible stupor in the meantime.

The soul-numbing effect of industrial technologies has been threatening to eradicate the world as we know it, our love and connection to it, Arendt's amori mundi, and art itself as our way of cultivating and passing on that love. The present work was propelled by an anxiety about 
the relationship between industrial technologies and art. It took up where Arendt and Heidegger left off when they pointed in the general direction of the arts as leading the path out of the techno-scientific mold. How, if at all, the arts could be the saving grace of the techno-human? To address this question, we had to look through technology to the symbolic mind, to being with language. But what is the linguistic destination, the destination of the symbolic being that extends into technological embodiment? Language as a symbolic way is a way to consciousness, a way of adding a vantage point to experience, the experience of the observer-witness. Those two modalities sometimes individuate such as in logical analysis where the observer is paramount and in dreaming where the witness comes to the fore. Most of the time they interpolate. However, those are two distinct modalities of consciousness that have yet to arrive at a totality of experience. It could be said that these are the two sides of the human experience, wakefulness and dream.

Marx located the site of (social) struggle in the symbolic representation of value commodity value represented by gold, gold represented by money - thus identifying symbolic layering in the construction of economic value. (Marx [1867] 1990) Heidegger found polemos in the way new (industrial) technology antagonizes the environment and enframes humanity in its conceptually-driven ways. In this work we looked at how symbolic layering and conceptual enframing were both given in language. We looked at how industrial technology hijacked (the) conceptual (of) language and, advancing its dissociative principle to isolate and reconnect, displaced vital movement with mechanics. This brought together Marx's and Heidegger's visions of society culture and technology in a way that could have vast political implications and can point to a new direction of rethinking cultural economy. This would entail redeploying a view of language not only as a mere top layer of representation (e.g. a word represents an object), but rather as the agglutinating force of symbolic being which unfolds in the intent to emancipate from 
both the inertia of matter and the imposition of thought. Caught between those two forces, the two banks of the same river, language gives not only the duality of polemic fight but also a way to discipline the body-mind through reason and swim away on the stream of poetry. Language is both the weapon that wounds and the medicine that can heal, as cinematic language intimates in new threatening and promising ways.

This project pulled together diverse ideas that were historically reluctant to converse. This was not a mere rearrangement of a new paragone of human activity that would prioritize the arts - it was a research in the ways art and technology could lead to another, more inclusive, more thought-full state of consciousness that would both fulfil the symbolic imperative and allow the human a freer relationship with technology that would harness and redeploy the energies lost in the process of mastering it and being mastered by it. This new state of consciousness would entail a leap to the simultaneous experience of two states of awareness - the witness and the observer. Dreaming awake and wakefully dreaming, humanity might just be able to move toward a new way of being in the world that would afford a freedom from the enframing power of technologies not by rejecting the conceptual (or, as is the case, embracing it religiously) but by redeploying it in the poetic, by redeploying techno-science in the artistic manifold. Cinema as art, by emulating the dreaming experience in wakefulness, promises to actualize dreaming awake as a way toward augmented consciousness through the technological augmentation of reality.

But this effect of cinema can be twofold. The moving image, as a neurological construct most evident in dream, is subject to a whole new level of technological probing - the level of recording and transmitting not merely the sensible, but the sub-sensible, the raw data of signals. Moreover, recording and transcribing the signals received by the brain is shaping up as the next level of recording. This kind of neuro-imaging allows understanding and control of the brain that 
can be both empowering and threatening, the latter in ways more sinister than the use of hypnotic filmmaking for mass control. When the poetic is subordinated to the rational, the beautiful to the utilitarian, we end up with products that use poetic language for indoctrination. Art is reduced to a secondary information tool at best, serving a know-it-all world of rational control. Redeploying the poetic as the living way of language not only recovers the position of the unknown as the source of knowledge but also restores the living environment of the scientific project as a way of knowing (and not merely a way of producing). The focus on awareness, on the singularity of experience, exercised by the artistic way opens up the possibility to reposition body-displacing industrial and hyper-industrial cyber technologies as tools, tools of consciousness. In material culture, where the symbolic meets the physical, technology has taken over by manipulating the human through and the symbolic. The appropriation of poetic value by consumer economy has led to commodity fetishism (Marx) displacing aesthetic value. The systematic enactment of the distancing-connecting signature of conceptual language isolates the human and fabricates the need for connecting devices. This process of individuation enforces a consumption standard that unifies in lieu of the poetic (individualism-as-uniqueness) communion (union in community). This is made possible by the focus on material needs that has fuelled techno-economy. The resulting technology has become symbolically superior to human with the promise of solving "intelligently" human problems of necessity. This intelligence is necessarily reduced to the mechanistic known that necessity belongs to. But when the collective attention shifts to the poetic which moves in the element of saying (Heidegger), and not telling, of listening (to the unknown) and not obeying (the known), new possibilities for a freer relationship to technology open up. As the source of language, poetry's destination becomes awareness. When awareness is the region of collective attention, the power of industrial technology to replace the embodied human becomes insignificant. (2) 
A cinema that foregrounds cinema as a kind of poetry. Cinema as lived poetry, advances on the destination toward awareness. Dreaming awake and waking in dreaming are ways of integrating poetry and reason not as useful steps in a rational project (art/poetry as a tool of emotional manipulation or the rational as the way of constructing the instrumentarium of art), but rather as body-mind integration unto awareness. The experience of cinema can trigger and forge such states of heightened awareness in keeping the view to the unknown open in the lived experience of poetry (as a (bodily) sensation and not only as (rational) conviction). By developing our dreaming attention while awake, cinema can facilitate a reunion with the dreaming body, the body of the mind, and reconnect it with the physical body, honing awareness in new ways that enable us to bypass the cognitive machinations of technology.

An example of how the artistic project can overwrite the technological imperative can be given with new tactile technologies. A touch screen which allows us to draw and paint with the finger not only mimics a return to traditional (body-bound) technologies but also realizes a fairy dream - the magic touch. Like a magic wand, our finger makes things happen through touch and yet with no connection to the materiality of (represented) things. This magic sanitization of the touch shifts the position of technology from technology as tool to technology as scene. The computer screen becomes the scene of a reality where anything becomes possible. The dream-like anything-is-possible reality of the movie screen now becomes a subject to our voluntary intervention. The passive viewer-dreamer becomes an active demiurge who can conjure up a world into existence. There are two major implications of this - it advances the cinematic sanitization expressed in the see-not-touch of pornography to incorporate the touch and minimize chance, and loss of control. Much of art in general and cinematic art in particular happens in the interaction with the material of the medium, the film stock which is much more prone to aesthetic chance and mistake than the controlled computer environment. Making a film on a computer 
promised results much closer to the imagined. However, certain techniques, such as traditional animation, remained contingent on physical contact. With the introduction of the stylus pen, the pen and brush strokes were not only incorporated in computer animation but also standardized now we could automatically texturize an image to give it an industrial-standard painted look. Screen touch technologies incorporated even the finger, the original, pre-technological artistic gesture, the last site of corporeal autonomy, giving the illusion of directness, of discarding one mediumatic layer, the tool, while in fact both the tool of the artist and the screen of the work were fused in one further distancing the artist from the final work.

This leads us to the second implication which is even more insidious - it redeploys the body in a pseudo-magic act and pacifies awareness with the illusion of control. In other words, it mimics the feelings and effects of poetic experience while minimizing the poetic through closing the cracks to the unknown, the socio-economic effects of which are explored at length in the Culture Thesis of Adorno and Horkheimer ([1947] 2002). The reflections of one's own limited technology-defined ideas and imaginations become substitutes for poetic insight and experience. Moreover, they become infinitely replayable/reproducible plunging the consumer in an addictive cycle of inducing the neurotic high mechanically reminiscent of the awe of the poetic experience.

So, how can this convenient entrapment of the artistic gesture in technologies be possibly overwritten without having to sacrifice the convenience? By redeploying the value of a symbolic product as a work of art rather than a work of technology and in doing so, redeploying the viewer's attention. For instance, what if the viewer's touch is used as the cinematic cut from one shot to another? This could be just another market gimmick, used automatically to bamboozle the viewer-user, or it could be taken as a challenge to rethink and widen cinematic language itself. 
Redeploying the artistic principle would mean shifting the values of use from product and process to insight and conscious experience. Insight requires generation of meaning and singularity of experience - an insight cannot be repeated. This shifts novelty from the consumer experience of a product to the awareness of the experience itself. Conscious experience requires presence and, again, precludes repetition, precludes the interpolation of the techno-mechanical process with the mind. Both insight and awareness require effort, they are challenging to thought, and not to the (physical) environment (as are industrial technologies). The reward of the ecstatic state (the experience of beauty) they cultivate is thus both more intense than the low-energy addictive satisfaction of industrial supply, and more elusive. It is contingent on the suspension of the known, i.e. on the suspension of the value of control that stems directly from conceptual thought sustaining industrial civilization. An industrial technology user fluent in poetic language is not the producer behind quantities of aesthetically (qualitatively) random pictures, for instance, filling the environment with the visual pollution of the hyper-industrial magic touch. A poet has a slow and deliberate eye in constant dialogue with visual reality - a camera, digital or not, may enter this dialogue but it may not lead it.

But how can the poetic eye be cultivated in an industrially dominated environment?

The easy answer would be education, and more specifically philosophical education, making thought more thoughtful, as Heidegger would have suggested, gaining the discipline to pace itself differently than the pace of technology so that it can evade hypnotic synchronization. Simply put, poetic language is about rhythm. A thoughtful fluency in rhythmic patterns would facilitate a possible distancing from the hypnotic ticking of industrial technology. However, a more probable way would be through harnessing the inertia of technology itself and pushing its development to the conceptual edge where it can no longer work without human conscious participation. This would mean redeploying the artistic principles in technological design, not as 
embellishments or shortcuts to consumers' unconscious, but as principles of design itself. Cinema offers the most fertile field for such developments because it is an art contingent on an industrial technology, a technology that constantly evolves, that has indeed usurped evolution and therefore an intervention into that evolution would be strategically warranted. In addition, as the language of new interactive moving image technologies demands viewer participation in immersive artificial realities, and in doing this, increasingly joins forces with neuroscience, cinema becomes the natural site for developing ways of challenging the user and mobilizing new ways of consciousness interactivity, where visual focus and attention shifts, for instance, could change the film scene even more magically than the magic touch. Thus the extreme simulacrum of future cinemas may be more than the site of apocalyptic dementia, it may also be the realm where the pornographic can recover its poetic energy and become once again the potent hand of Atum conjuring a new dimension to the world assembled by a new type of dreaming-awareness-inwakefulness and apprehended by a new wakeful-consciousness-in-dream.

Closing this work by opening the question of poetic design is a call to redeploying oneiric energies in ways that bring back the unknown as the context of all our efforts, and make thought more thoughtful not only in wakefulness but also in its native realm - dream.

In scanning such a syncretic field as cinema, and using a syncretizing method such as Tensegrity (allowing for the integration of the different ways of thinking, practising and theorizing that run and cross on that field), this work has necessarily realigned a number of theoretical relationships, weaving its own dynamic net in the process. Thus there are findings on many levels of the work and perhaps some of the less conspicuous ones would turn out to be most important. However, if we have to over-synthesize, the finding that gives blood to all others is the new positioning of poetic language as not only one of the two ways of language, but also the view of the poetic as the source of language itself, as the linguistic opening to the unknown. It resolves 
the tension between the mental and the physical as that which energizes both the hand of Atum and the mouth of Ptah, thus interpolating them in vital ways. The vital force that animates both the physical (Atum,) and the symbolic (Ptah) remains poetry. The concept-driven industrial imperative moves toward suppressing the poetic in both life (as the animator of the physical body) and soul (as the animator of the mind) by reducing the former to its functioning and functionality (those aspects potentially available to technological mimesis), and the latter to a metaphor of the mind-as-function-of-the-brain (again, an aspect of corporeal functionality and potentially imitable by technology). The zombification of the body, the ever living dead, and the ghostification of the soul, the ever haunting mind, are not only products of the techno-scientific imagination, they are emblematic of its strategies to neutralize and assimilate the poetic. On the clearing offered by industrial technologies, cinema as an art form, offers a new way of redeploying poetic energies, a way that circumvents the industrial effort to absorb embodied awareness. The zombified bodies of pornography, forever fornicating in the ghost universe of cinema, are offered an unlikely redemption by the very medium that made their original embodiment redundant. The very ghost-like character of cinema is also that which opens it as the site of poetic embodiment. Cinema as art is a kind of poetic embodiment that can fling open the doors the industrial imperative shuts tight, and do that from within the tenets of that imperative. As such, cinema is the gift of industrial technology. 


\section{Bibliography}

Abbot, Edwin. 1884. Flatland: A Romance of Many Dimensions. New York, NY: Basic Books, 2002.

Arendt, Hannah. 1958. The Human Condition. Chicago: University of Chicago Press, 1998.

Arendt, Hannah. 1971. The Life of the Mind. Orlando, FL: Harcourt, Inc., 1978.

Aristotle. 350 BC. Nicomachean Ethics. Indianapolis: Hackett Publishing Company, 1999.

Artaud, Antonin. 1938. The Theater and Its Double. New York, NY: Grove Press, 1958.

Ayeza, Jozefina. Interview with Wim Delvoye. http://www.lacan.com/frameVII11.htm, $\underline{2013}$

Barthes, Roland. 1970. "The Third Meaning: Research Notes on Several Eisenstein Stills." The Responsibility of Forms: Critical Essays on Music, Art, and Representation. Berkeley: University of California Press, 1985.

Bazin, André. What is Cinema? Trans. and ed. Hugh Gray. Vol 1. Berkeley: University of California Press, 1967.

Belloi, Livio. "Vitraux aux mouvantes pierreries: le film peint (Marcelle Thirache, Stan Brakhage)." L'œuvre en morceaux. Esthétiques de la mosaïque. Livio Belloï and Michel Delville (Eds.). Paris-Bruxelles, Les Impressions Nouvelles, 2006.

Benjamin, Walter. 1936. The Work of Art in the Age of Mechanical Reproduction. http://www.marxists.org/reference/subject/philosophy/works/ge/benjamin.htm, 2014 
Benjamin, Walter. 1940-1088. The Arcade Project. Cambridge, MA \& London, England: The Belknap Press of Harvard University Press, 2002.

Bergson, Henry. 1911. Matter and Memory. New York: The Macmillan Company, 1929.

Bohm, David. 1980. Wholeness and the Implicate Order. London and New York: Routhledge Classics, 2002.

Bourdieu, Pierre. The Field of Cultural Production: Essays on Art and Literature. New York, NY: Columbia University Press, 1993.

Bordwell, David. “A Case for Cognitivism,” in IRIS No.9, Spring 1989.

Borges, Jorge Luis. “On Exactitude of Science.” A Universal History of Infamy. London: Penguin Books, 1975.

Borges, Jorge Luis. 1957. The Book of Imaginary Beings. London: Penguin Books, 2005.

Bachelard, Gaston. 1960. The Poetics of Reverie. Boston: Beacon Press, 1971.

Brown, Bill. "Thing Theory." Critical Inquiry, Vol. 28, No.1, Things. (Autumn, 2001), pp.1-22.

Cavell, Stanley. The World Viewed: Reflections on the Ontology of Film. Cambridge, MA: Harvard University Press, 1979.

Carman, Taylor. "The Body in Husserl and Merleau-Ponty.” PHILOSOPHICAL TOPICS, Vol.27, NO.2, Fall 1999.

Chomsky, Noam. Language and Mind. Cambridge: Cambridge University Press, 2006

Coonan, Clifford. "Peter Greenaway says cinema is dead." Variety. October 9, 2007.

Damasio, Antonio. The Feeling of What Happens. Orlando: Harcourt, 1999.

Deleule, Didier. "The Living machine: Psychology as Organology." Crary, Jonathan \& Kwinter, Sanford (Eds.). Incorporations. New York: ZONE, 1992. 
Deleuze, Gilles \& Guattari, Felix. 1991. What is Philosophy? New York \& London: Columbia University Press, 2003.

Deleuze, Gilles \& Guattari, Felix. 1972. Anti-Oedipus: Schizophrenia and Capitalism. Minneapolis: University of Minnesota Press, 1983.

Deleuze, Gilles. 1986. Cinema 1: The Movement-Image. Minneapolis: University of Minnesota Press, 1997.

Deleuze, Gilles. 1989. Cinema 2: The Time-Image, Minneapolis: University of Minnesota Press, 1997.

Derrida, Jacques. (1978). Writing and Defference. London: Routhledge. (1967).

De Saussure, Frederick. 1972. Course in General Linguistics (3rd ed.). (R. Harris, Trans.). Chicago: Open Court Publishing Company, 1986.

Di Pippo, Alexander Ferrari. The Concept of Poiesis in Heidegger's An Introduction to Metaphysics. In: Thinking Fundamentals, IWM Junior Visiting Fellows Conferences, Vol. 9: Vienna, 2000.

Eisenstein, Sergei. 1949. Film Form: Essays in Film Theory. San Diego, New York, London: A Harvest Book, Harcourt Brace \& Company, 1977.

Elder, Bruce R. Harmony and Dissent: Film and Avant-garde Art Movements in the Early Twentieth Century. Waterloo, ON: Wilfrid Laurier University Press, 2010.

Feuerbach, Ludwig. 1841. The Essence of Christianity. Mineola, NY: Dover Publications, 2008.

Feyerabend, Paul. 1975. Against Method. New York \& London: Verso, 2002.

Flaxman, Gregory. The Brain is the Screen: Deleuze and the Philosophy of Cinema. Minneapolis: University of Minnesota Press, 2000.

Foucault, Michel. 1961. Madness and Civilization. New York, NY: Vintage Books, 1988. 
Foucault, Michel. 1976. The History of Sexuality: An Introduction. New York, NY: Vintage Books, 1990.

Fried, Gregory. Heidegger's Polemos: From Being to Politics. New Haven \& London:Yale University Press, 2000.

Fromm, Erich. The Forgotten Language; An Introduction to the Understanding of Dreams, Fairy Tales, and Myths. New York, NY: Henry Holt \& Co., 1974.

Frampton, Daniel. Filmosophy. New York: Columbia University Press, 2006.

Fuller, Buckminster Richard. Your Private Sky: Discourse. Zürich: Lars Müeller Publishers, 2001.

Graham, Daniel W. "Heraclitus." The Stanford Encyclopedia of Philosophy (Summer 2011 Edition). Edward N. Zalta (ed.). URL = http://plato.stanford.edu/archives/sum2011/entries/heraclitus/, 2014.

Haraway, Donna. Simians, Cyborg, and Women: The Reinvention of Nature. New York: Routhledge, 1991.

Heidegger, Martin. 1971. On the Way to Language. New York: HarperCollins Publishers, 1982.

Heidegger, Martin. 1943. "On the Essence of Truth." Basic Writings. San Francisco: Harper Collins Publishers, 1977.

Heidegger, Martin. 1954. "The Question Concerning Technology." Basic Writings. San Francisco: Harper Collins Publishers, 1977.

Heidegger, Martin. 1960. "The Origin of the Work of Art ." Basic Writings. San Francisco: Harper Collins Publishers, 1977.

Heidegger, Martin. 1927. Being and Time. Albany, NY: State University of New York Press, 1996. 
Heraclitus. The Cosmic Fragments. (G.S. Kirk Trans.) Cambridge, MA: Cambridge University Press, 2010.

Horkheimer, Max \& Adorno, Theodor W. 1947. Dialectic of Enlightenment: Philosophical Fragments. Stanford University Press, 2002.

Husserl, Edmund. 1948. Experience and Judgment: Investigations in a Genealogy of Logic. Evanston: Northwestern University Press, 1974.

Ihde, Don. Philosophy of Technology (Paragon Issues in Philosophy). London \& New York: Continuum International Publishing Group, 1993.

Donald E. Ingber. "Cell structure and hierarchical systems biology." Cell Science. April 1, 2003 J. 116, 1157-11

Junger, Ernst. 1934. On Pain. New York: Telos Press Publishing, 2008.

Kaes, Anton. Shell Shock Cinema: Weimar Culture and the Wounds of War. Princeton, New Jersey and Oxford, Oxfrdshire: Princeton University Press, 2009.

Kant, Immanuel. 1974. Observations on the Feeling of the Beautiful and Sublime. Berkley and Los Angeles: University of California Press, 1991.

Kant, Immanuel. 1790. The Critique of Judgement. London: MacMillan and Co., Limited, 1914.

Kant, Immanuel. 1800. Logic. Mineola, NY: Dover Publications, 1988.

Kristeva, Julia. 1974. Revolution in Poetic Language. New York, NY: Columbia University Press, 1984.

Lacan, Jacques. The Language of the Self: The Function of Language in Psychoanalysis. Baltimore: The Johns Hopkins University Press, 1968.

Lacan, Jacques. (1953-1954). "The Mirror-phase as Formative of the Function of the I." Slavoj Zizek (Ed.). Mapping Ideology. London \& New York: Verso, 1994. 
Logan, Robert. The Extended Mind: The Emergence of Language, The Human Mind, and Culture. Toronto, Buffalo, London: University of Toronto Press, 2007.

Lorca, Federico-Garcia. 1933. Theory and Play of the Duende. http://www.poetryintranslation.com/PITBR/Spanish/LorcaDuende.htm, 2013.

Lyotard, Jean-Francois. Libidinal Economy. Bloomington: Indiana University Press, 1993.

Lyotard, Jean-Francois. The Inhuman: Reflections on Time. Stanford: Stanford University Press, 1991.

MacKinnon, Catherine. Only Words. Cambridge, MA: Harvard University Press, 1993.

Marcuse, Herbert. 1969. An Essay on Liberation. Bostan, MA: Beacon Press, 1971.

Marcuse, Herbert. 1998. Technology, War and Fascism. London and New York: Routledge, 2004.

Marks, Laura U. "Information, secrets, and enigmas: an enfolding-unfolding aesthetics for cinema." Screen 50:1. Spring 2009.

Marx, Karl. 1867. Capital: A Critique of Political Economy. Volume One. London: Penguin Classics, 1990.

Maturana, Humberto R. \& Varela, Francisco J. 1972. Autopoiesis and Cognition: The Realization of the Living. Dordrecht, Holland: D. Reidel Publishing Company, 1980.

McLuhan, Marshall. Understanding Media: The Extensions of Man. New York, Toronto, London: McGraw-Hill Book Company, 1964.

McLuhan, Marshall \& McLuhan, Eric. 1988 Laws of Media: The New Science. Toronto: University of Toronto Press, 1992. 
McLuhan, Herbert Marshall. 1951. The Mechanical Bride: Folklore of Industrial Man. Corte Madera: Gingko Press, 2002.

Mekas, Jonas. Movie Journal: The Rise of the New American Cinema, 1959-1971. London, UK: Macmillan Company, 1972.

Mekas, Jonas. Jonas Mekas' Serpentine Garden Marathon. Dazed, 2012: http://www.dazeddigital.com/artsandculture/article/11782/1/jonas-mekas-serpentine-gardenmarathon

Mekas, Jonas \& Silverman, Jason. "Short Films from a Long Life." Interview in Wired, December 19, 2006.

Merleau-Ponty, Maurice. The Visible and the Invisible. Evanston, Illinois: Northwestern University Press, 1968.

Merleau-Ponty, Maurice. Signs. Evanston, Illinois: Northern University Press, 1964.

Merleau-Ponty, Maurice. 1945. Phenomenology of Perception. London and New York: Routledge, 2002.

Metz, Christian. 1974. Film Language: A Semiotics of the Cinema. Chicago: University of Chicago Press, 1991.

Metzinger, Thomas. The Ego Tunnel. New York, NY: Basic Books, 2009.

Müller, Max (Ed). Sacred Books of the East, Vol 39. London: Oxford University Press, 1891.

News Editorial. CURRENT SCIENCE, VOL. 79, No. 1, 10 July 2000

Nietzsche, Friedrich. 1889. Twilight of the Idols. Oxford: Oxford University Press, 1998.

Nietzsche, Friedrich. 1886. Beyond Good and Evil. London: Penguin Classics, 2003. 
Nietzsche, Friedrich. 1878. Human, All Too Human: A Book for Free Spirits. Cambridge: Cambridge University Press, 2004.

Nietzsche, Friedrich. 1872. Razhdaneto na tragediata [The Birth of the Tragedy]. Sofia: Izdatelstvo Naouka i Izkustvo, 1990.

Nishimoto, Shinji; Vu, An T.; Naselaris, Thomas; Benjamini, Yuval; Yu, Bin and Gallant, Jack L. Reconstructing Visual Experiences from Brain Activity Evoked by Natural Movies. Current Biology, Volume 21, Issue 19, 1641-1646, 22 September 2011.

Oakes, Lorna. Ancient Egypt:: An Illustrated Reference to the Myths, Religions, Pyramids and Temples of the Land of the Pharaohs. New York: Barnes \& Noble, 2003.

Plato. 380 BC. The Republic. Indianapolis: Hackett Publishing Company, 2004.

Plato. 360 BC. Symposium. http://classics.mit.edu/Plato/symposium.html, 1994-2009.

Plato (2). 360 BC. Phaedo. http://classics.mit.edu/Plato/phaedo.html, 1994-2009.

Ramachandran, V. S. 1998. Phantoms in the Brain: Probing the Mysteries of the Human Mind. New York: HarperCollins Publishers, 1999.

Richardson, William J. 1963. Heidegger: Through Phenomenology to Thought. Bronx, NY: Fordham University Press, 2003.

Richter, Gerhard. Thought-Images: Frankfurt School Writers' Reflections from Damaged Life. Stanford, California: Stanford University Press, 2007.

Searle, John. The Rediscovery of the Mind. Cambridge: MIT Press, 1994.

Steele, Robert and Logan, Robert. "Report on a Study of Light Variables Measured as a Function of Time." The Journal of The Society of Cinematologists, Vol. 4/5 (1965/1965). pp. 3754. 
Trott, Elizabeth Anne. "Music, Meaning, and the Art of Elocution." The Journal of Aesthetic Education. Volume 24. No 2. Summer 1990.

Varela, Francisco J.. "Neurophenomenology: A Methodological Remedy for the Hard Problem". Journal of Consciousness Studies, 3. No. 4. 1996. pp. 330-49.

Vertov, Dziga. 1919. "WE: Variant of a Manifesto." Kino-Eye: The Writings of Dziga Vertov. Ed. Anette Michelson Berkley and Los Angeles, CA: University of California Press, 1984.

Virilio, Paul. War and Cinema. London and New York: Verso, 1989.

Virilio, Paul. Open Sky. London and New York: Verso, 1997.

Orson Welles. International Film Annual, No. 2. Edited by William Whitebait. London: John Calder, 1958.

Wilkinson, Richard H. The Complete Gods and Goddesses of Ancient Egypt. Thames \& Hudson, 2003.

Wimpenny J.H., Weir A. A., Kacelnik A. "New Caledonian crow use tools for nonforaging activities." Anim Cogn. 2011 May. 14 (3): 459-64. epub. 2010 Dec 24.

Zhabilova, Kamelia. "The Shadows of God. A Linguistic Critique of Nietzsche's Metaphysics.” (“Сенките на Бога. Филологическа критика на метафизиката при Ницше”). The Language of Being. (Езикът на Битието). Sofia: Institude for Philosophical Research.(2010). http://vasev.org/enb/kjabilova/ (2013) 


\section{Appendix}

\section{The Eyes that Stop the Train}

(A Moving Image Installation)

\section{Project Description}

In a corridor-like space, a sequence of six back-lighted panels (36" x 24") of lenticular animation face six computer monitors. Each lenticular print is a six-frame animation of a landscape shot from a train moving at a very high speed (rendering the imagery abstract).

On the opposite wall, six corresponding computer monitors screen the full sequence as shot in the original video format. The sequence is looped on in a consecutive manner that makes the monitors appear as the windows to a continuous fast-moving landscape.

While the viewer's angle and position have no bearing on the speed or duration of the video streaming (as is the case in standard/traditional moving image technologies), the movement of the lenticular animation is activated by the angle at which the lenticular print is viewed, it is entirely dependent on the position of the viewer who has full control over duration.

The corridor leads to a space where a projection of the film The Eyes that Stop the Trains with full sound, is running. The film is edited to John Cage's Bachhanale. (see film synopsis). On the opposite wall, a single frame from the film blown up and cut into many frames that are printed out in letter=size paper each and assembled in a 2-D art-work reminiscent of Peter 
Kubelka's celluloid installations. A video assembly of the frames into a highly abstract video is projected in time next to the 2-d spatial spread.

The Eyes that Stop the Trains harnesses the capacity of the lenticular medium to conduct an analog-type of interactivity where reciprocal communication depends on the physical involvement of the viewer in order to question the modality of film-time and its phenomenological relation to physical movement. The installation aims to immerse the viewer in the paradoxes of movement and rest, of space and time, as enacted by a range of moving image technologies.

\section{Project Synopsis}

The Eyes that Stop the Train is a trans-media installation exploring the semantic, formal and visceral implications of moving image technologies and their capacity to affect viewers' space-time.

Live cinema is, in its most basic photographic premise, an abstracted mirror of life. Abstracted from the corporeality of an event which enables the transportability of that event through space and time, invoking it beyond its singular physical manifestation. The viewer can watch a replay of an event almost as though s/he is witnessing it live. The video component of the installation pushes this understanding of the cinematic image to the limit - a durational shot of a POV onto a landscape from a moving train is screened consecutively on six monitors in a manner that simulates the sight seen from the windows of a moving train. This mediumatic transposition of a live moment into a gallery space invites the viewer to explore the experience of the medium itself, its reality, both presencing and reflective, the perceptual possibilities and limitations it 
imposes. Akin to traditional cinema, the video sequence is best experienced in passive viewing. On the opposite wall of the corridor-like space, six lenticular prints of the same video sequence invite the viewer to walk and move in order to animate the image. Suddenly, the train can't move unless the viewer moves. Is the world moving around us or are we moving within it? What do moving image technologies say about our experience of movement?

The second part of the installation explores the cinematic as montage. A poetic interpretation of the theme and original footage of the view from a moving train is contrasted with a structural de-construction of the (spatial) shot turned into an image-in-time and materialized as a 2-D carbon copy thanks to digital technologies. The latter is in response to Peter Kubelka's questioning of the ways celluloid and paper (editing) correlate in montage techniques, isolating digital numerization. I am pushing the argument in exploring how far digital technologies can simulate not only perceptual (the video sequence) and mental (the film) experience of the moving image, but also the analog modalities of moving image technology itself.

This installation is a manifold questioning of cinema-without-cinema. The only missing experience is the celluloid projection, the beginning of cinema. From beyond cinema's death with the birth of the digital, it invites a meditation on the cinematic beyond the cinematic.

\section{Film Synopsis}

Moments of life lived, missed, remembered and forgotten, as glimpsed through the window of a moving train.

A view on thought and vision playing out the film of life - eyes looking through the window of a moving train. This film invokes the process of memory, the way thought snippets interject perception. The moving train $=>$ time $=>$ the moving image. The beginning of cinema: 
Arrival of a Train at La Ciotat seen from the POV of the passenger. The passage of time seen through the ears of John Cage: Bacchanale - the sound of life dynamic and chaotic, a thrust forth in time reversible/re-playable unto death. 